\title{
Rhenium-osmium geochronology and isotopic systematics in New Zealand source rocks and oils
}

By

Enock Kipngeno Rotich

A thesis submitted to the Victoria University of Wellington in fulfilment of the requirements for the degree of

Doctor of Philosophy 


\begin{abstract}
The Re-Os radiogenic isotope system has over the past three decades been successfully applied to organic-rich sedimentary rocks and oils as a geochronometer and geochemical tracer. The Re-Os geochronometer has provided a direct way of constraining the depositional age of organic-rich sediments as well as the timing of oil generation events. Osmium isotopic compositions have further been utilised in understanding past climatic, oceanographic and geological events recorded in sediments, and in correlating oils to their source. Thus far, however, Re-Os studies of organic-rich sediments have mainly focused on marine black shales where Re and Os are primarily sourced from seawater. The work presented in this thesis seeks to investigate factors controlling Re-Os systematics and potential for geochronology in a range of fluvio-deltaic coaly rocks and terrestrial organic matter-dominated marine sediments, and associated oils from New Zealand's Taranaki and East Coast basins.
\end{abstract}

The Re-Os data presented here yield the first radiometric age for the late Paleocene Waipawa Formation $(57.5 \pm 3.5 \mathrm{Ma})$, a marine sedimentary unit that was formed by episodic input of large amounts of terrestrial woody plant matter resulting in high average sedimentation rates of up to $\sim 10.6 \mathrm{~cm} / \mathrm{ky}$. This age is consistent with available biostratigraphic age determinations. The formation possesses Re $(38.9 \pm 17.6 \mathrm{ppb})$ and Os $(526 \pm 75.8 \mathrm{ppt})$ concentrations similar to those found in typical marine sediments containing amorphous organic matter deposited under much lower sedimentation rates. This indicates that organic matter type and sedimentation rate may not play a significant role in sequestration of these elements in organicrich sediments. Unlike the Waipawa Formation, coals and coaly mudstones with varying degrees of marine influence (purely terrestrial to strongly marine-influenced) from the Rakopi, North Cape, Farewell and Mangahewa formations record low average Re (0.37 $\pm 0.25 \mathrm{ppb})$ and Os $(24.5 \pm 11.9 \mathrm{ppt})$ concentrations. These concentrations are up to two orders of magnitude lower than those of similarly marine-influenced coals from the Matewan coalbed, USA, suggesting that Re and Os enrichment in coals does not simply correlate with the level of marine influence; the timing and nature of the marine influence, as well as chelation ability of organic-rich sediments, are equally important.

The initial ${ }^{187} \mathrm{Os} /{ }^{188} \mathrm{Os}(\mathrm{Osi})$ values for the Waipawa $(\sim 0.28)$ and underlying Whangai $(\sim 0.36)$ formations are broadly similar to those reported for coeval pelagic sediments from the central 
Pacific Ocean, further constraining the low-resolution marine ${ }^{187} \mathrm{Os} /{ }^{188} \mathrm{Os}$ record of the Paleocene. A broad correlation between this record and global temperature $\left(\delta^{18} \mathrm{O}\right.$ and TEX 86$)$ and carbon isotope $\left(\delta^{13} \mathrm{C}\right)$ records is observed from the middle Paleocene to early Eocene, which is inferred to reflect climate-modulated changes in continental weathering patterns. Unlike the marine sediments, significant variations are noted in the Osi of the Taranaki Basin coaly rocks. These are linked to depositional and diagenetic conditions, degree of water connectivity with the open ocean, and sediment source. The large variations in Osi values combined with small ranges in ${ }^{187} \mathrm{Re} /{ }^{188} \mathrm{Os}$ ratios and relatively young ages are considered as factors that hindered development of Re-Os isochrons in these rocks.

Crude oils sourced from the Taranaki coals and coaly mudstones also record low average Re $(0.31 \pm 0.09 \mathrm{ppb})$ and Os $(14 \pm 7.6 \mathrm{ppt})$ concentrations and have ${ }^{187} \mathrm{Re} /{ }^{188} \mathrm{Os}$ and ${ }^{187} \mathrm{Os} /{ }^{188} \mathrm{Os}$ ratios that do not correlate on an isochron diagram. The lack of an isochron fit for these oils is mainly attributed to a large variation in Osi values $(0.47-1.14)$ resulting from the heterogeneous nature of their potential Rakopi and North Cape coaly source rocks and a lengthy (20 Myr) oil generation event. These Osi values, however, overlap with ${ }^{187} \mathrm{Os} /{ }^{188}$ Os values for the potential source rocks at the time (ca.10 Ma) of oil generation (0.38-1.26), suggesting that Os isotopes may be utilised in tracing these oils. Crude oils that have potentially been sourced from the Waipawa and Whangai formations record much higher Re $(2.86 \pm 1.92 \mathrm{ppb})$ and Os (166 \pm $142 \mathrm{ppt}$ ) concentrations than the coaly-sourced oils, and show Os isotopic compositions that either correlate with those of their potential source rocks (e.g., oil Osi $=\sim 0.63$ compared with Waipawa Formation ${ }^{187} \mathrm{Os} /{ }^{188} \mathrm{Os}=0.48-0.68$ at time of oil generation) or differ due to likely secondary alteration processes within the reservoir such as thermochemical sulfate reduction (TSR). 


\section{Dedication}

In the course of writing this thesis, my wife, Caroline, gave birth to our first-born son, Miles. It is to them that I dedicate this work. 


\section{Acknowledgements}

First and foremost, I would like to thank my supervisors, Monica Handler and Sebastian Naeher, for their guidance, encouragement and support throughout this project. Having good supervisors is the key to a positive experience in an otherwise tumultuous $\mathrm{PhD}$ journey. Monica and Sebastian have been very dedicated to their roles, sharing their expertise ever so willingly and being open to discussing my thoughts and ideas. Thanks, Monica, for securing the funding for this project, for all the help with lab work at VUW and for always looking out for my welfare. Thanks, Sebastian, for sending back the thesis drafts in lighting speeds and for all the help setting up the lab work at GNS, getting samples from the National Core Store in Featherston, introducing me to people at GNS Science and general discussions about life beyond the PhD. I have been lucky to have you two as my supervisors.

This project was only made possible through funding from the Ministry of Business, Innovation and Employment (MBIE), New Zealand, as part of the GNS Science-led programme "Understanding petroleum source rocks, fluids, and plumbing systems in New Zealand basins: a critical basis for future oil and gas discoveries" (Contract C05X1507). This funding covered my PhD scholarship, all laboratory work undertaken at Victoria University, GNS Science and Durham University and participation at the 2019 and 2020 Goldschmidt conferences. I would also like to thank Victoria University faculty strategic research grant for providing additional funding to cover travel expenses to Barcelona for the 2019 Goldschmidt conference.

I would like to acknowledge the immense support that I got from Richard Sykes at GNS Science and Dave Selby at Durham University throughout this $\mathrm{PhD}$. Richard has been very supportive from the beginning, taking so much time in making sure that I got the best possible samples and meticulously reviewing my manuscripts. Dave made sure that my stay in Durham during my two lab visits was beyond reproach. He gave me all the necessary training to be able to handle the lab work on my own and made sure that I always got the first priority when it came to the availability of lab space and instrument. I would also like to thank Chris Hollis at GNS Science for thoughtful comments and insights into my first paper, and for providing support during the sampling of the Waipawa and Whangai formations.

During this project, I have also benefited a lot from people in the different labs I worked in. These include Antonia Hoffman (Durham), Geoff Nowell (Durham), Zeyang Liu (Durham), 
Junjie Liu (Durham), Bruce Charlier (VUW), Luisa Ashworth (VUW), Roger Tremain (GNS), Andy Philips (GNS) and Joan Fitzgerald (GNS). I would like to also acknowledge CRL Energy Ltd for undertaking the sulfur analyses.

To colleagues and friends at VUW, thank you for the unending support. Thanks especially to office mates Rubianca Benavidez, Anh Dang, Omid Khazaeian, Mohammed Namdar, Dinah Fieman and Sholeh Mousavipour for all the good conversations and fun times. Not forgetting Marcel, Jurgen and Leo for words of encouragements along the corridors and common rooms of VUW.

Finally, and most importantly, I would like to thank my family for being a constant source of support, inspiration, encouragement and unconditional love. Thanks to my parents and siblings for having supported me financially and spiritually throughout my educational endeavour. Special thanks to my wife, Caroline, for always being there through the hard times. 


\section{Table of Contents}

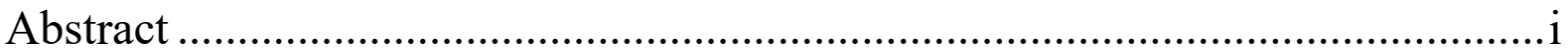

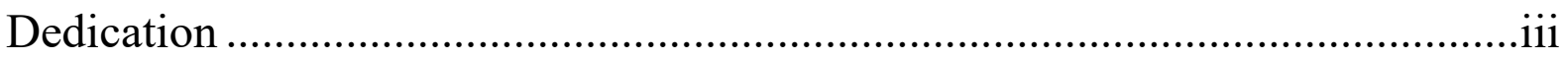

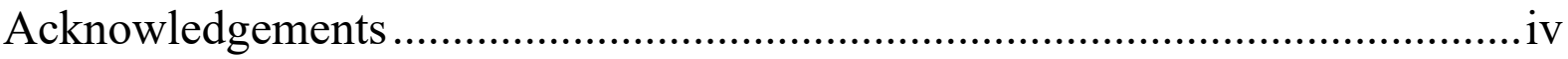

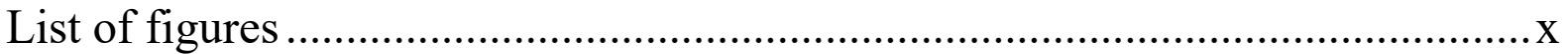

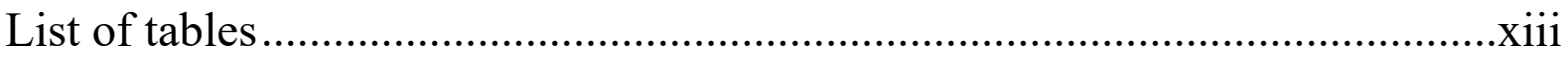

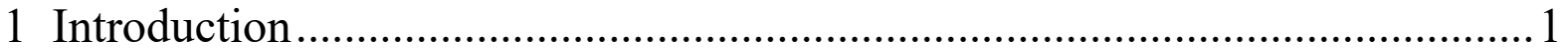

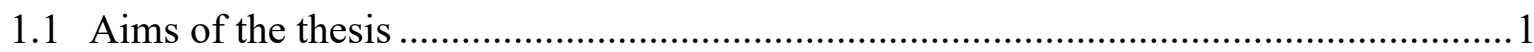

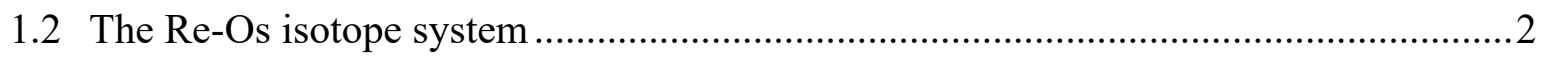

1.3 Re-Os systematics and geochronology in organic-rich sedimentary rocks ..................6

1.3.1 Re and Os sequestration in organic-rich sedimentary rocks .............................6

1.3.2 Re-Os geochronology of organic-rich sedimentary rocks ................................. 8

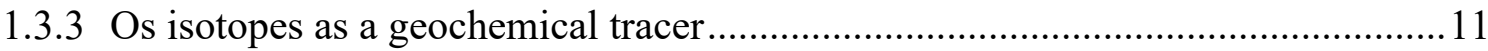

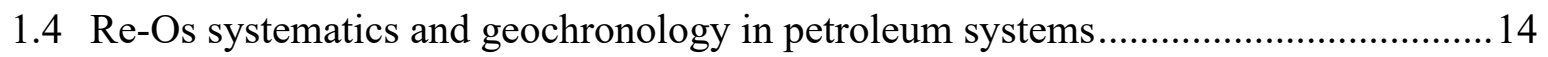

1.4.1 Residency and fractionation of Re and Os within crude oil.................................15

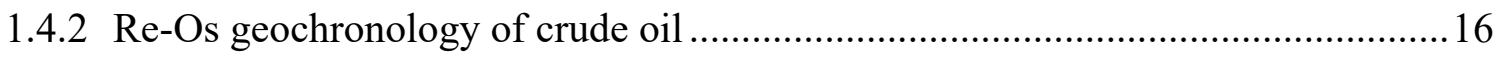

1.4.3 Os isotope fingerprinting of oils to inferred source rocks.................................... 19

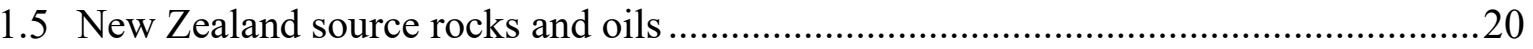

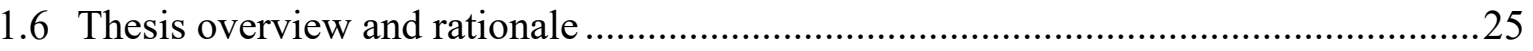

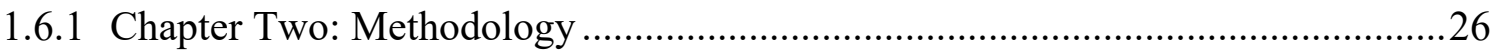

1.6.2 Chapter Three: Re-Os geochronology and isotope systematics, and organic and sulfur geochemistry of the middle-late Paleocene Waipawa Formation, New Zealand: Insights into early Paleogene seawater Os isotope composition. .26

1.6.3 Chapter Four: Depositional influences on Re-Os systematics of late CretaceousEocene fluvio-deltaic coals and coaly mudstones, Taranaki Basin, New Zealand 27

1.6.4 Chapter Five: Re-Os systematics in marine and terrestrial oils from the Taranaki and East Coast basins, New Zealand.................................................................2 27

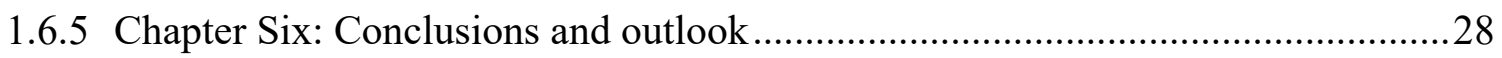




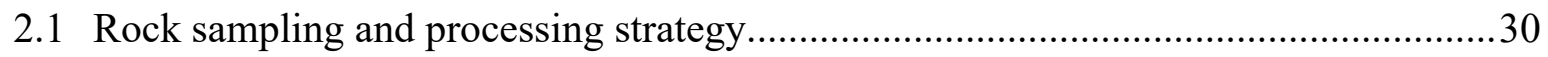

2.1.1 Sampling to evaluate Re-Os systematics in different depositional settings...........30

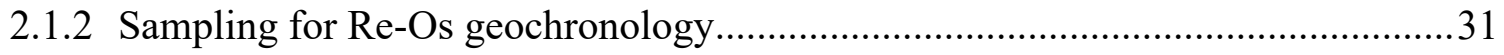

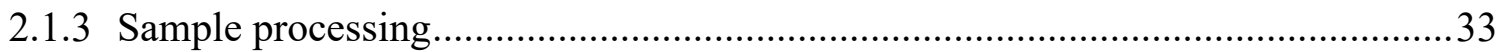

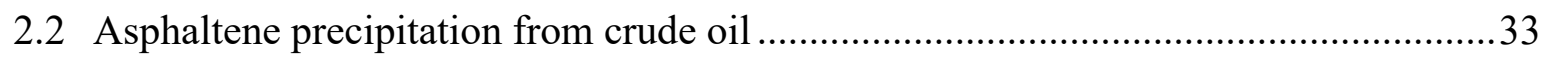

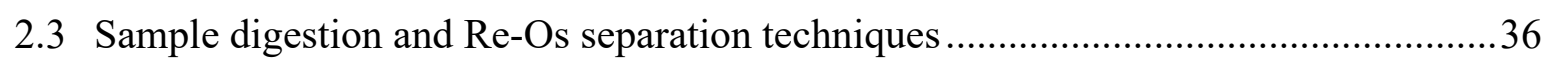

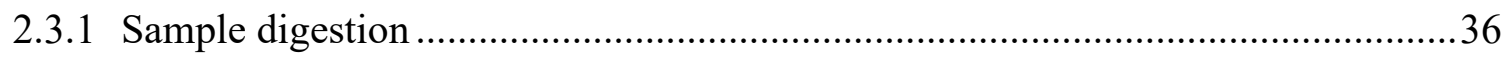

2.3.2 Osmium separation and purification chemistry ….......................................... 40

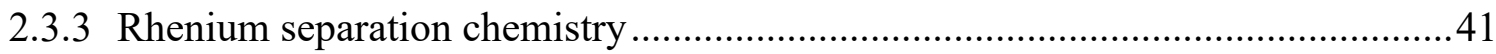

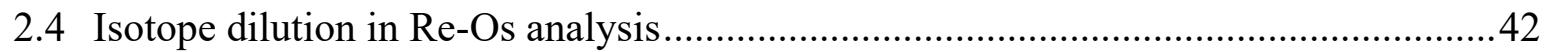

2.5 Mass spectrometric techniques for Re-Os isotope analysis....................................47

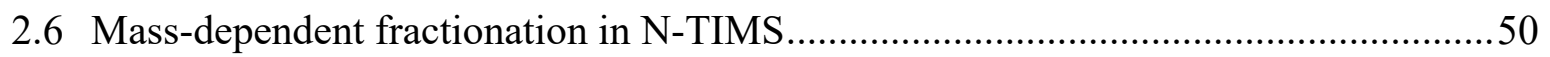

2.7 Linear regression in Re-Os isochron geochronology .............................................51

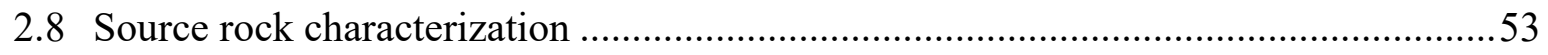

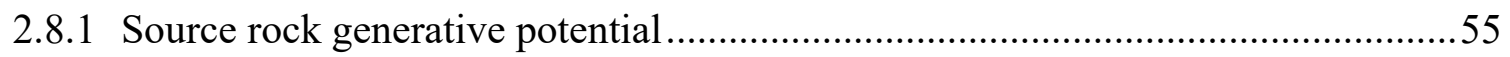

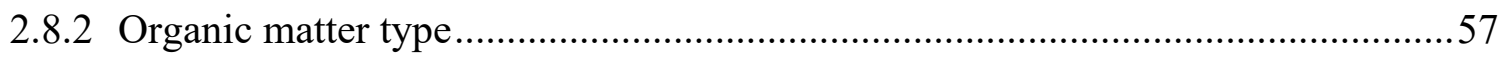

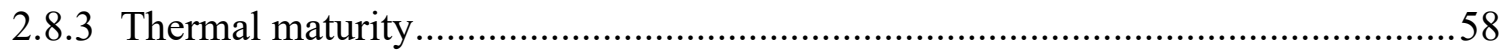

3 Re-Os geochronology and isotope systematics, and organic and sulfur geochemistry of the middle-late Paleocene Waipawa Formation, New Zealand: Insights into early Paleogene seawater Os isotope composition .....59

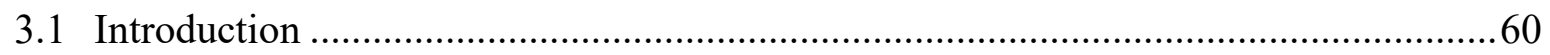

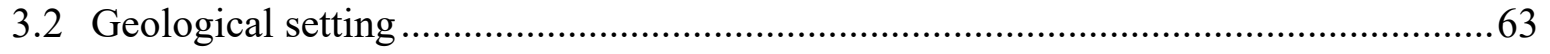

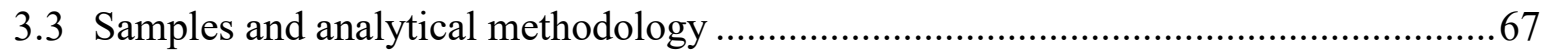

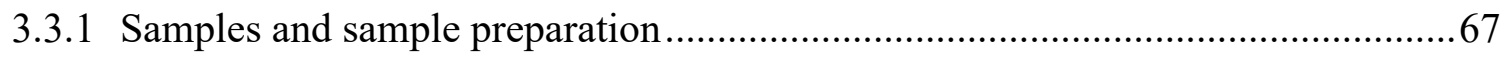

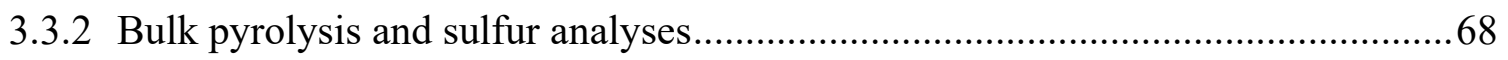

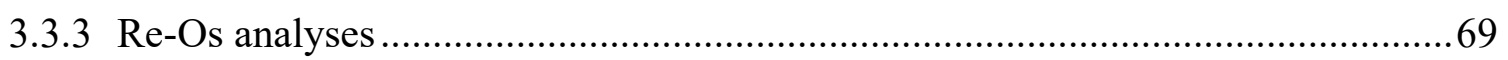

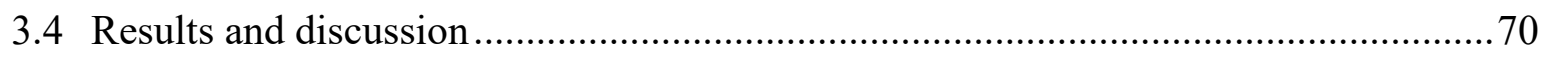

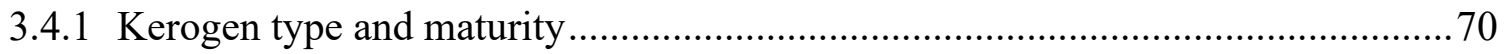

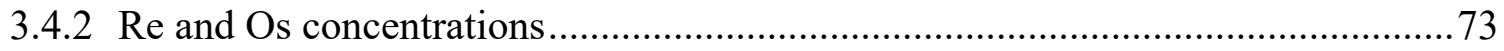

3.4.3 Re and Os uptake and fractionation in the Waipawa and Whangai formations.....75 


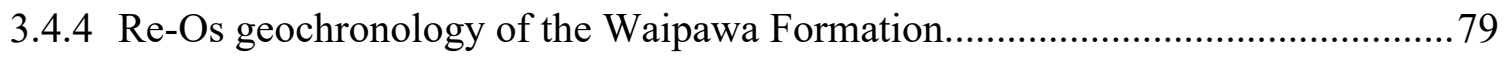

3.4.5 Comparison of the Waipawa Formation Re-Os and biostratigraphic ages ............82

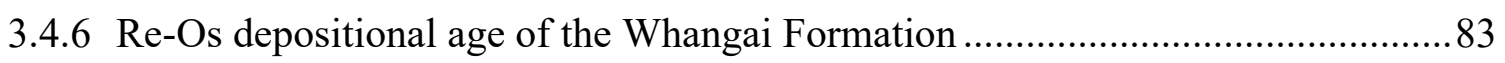

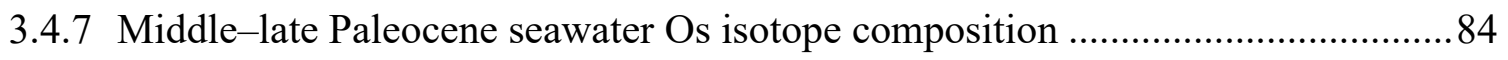

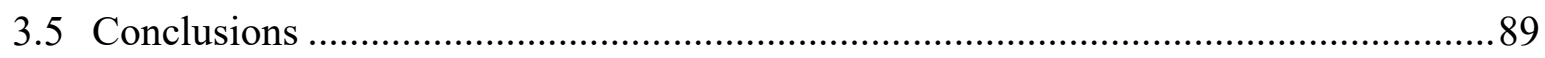

4 Depositional influences on Re-Os systematics of late Cretaceous-Eocene fluvio-deltaic coals and coaly mudstones, Taranaki Basin, New Zealand.....90

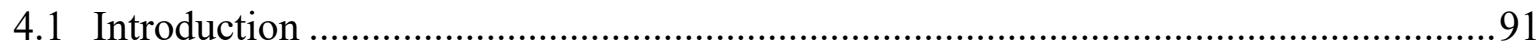

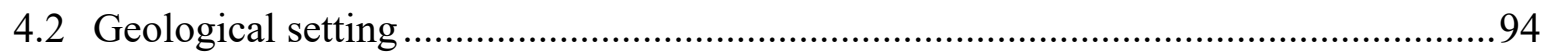

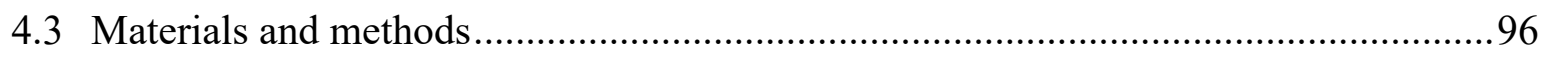

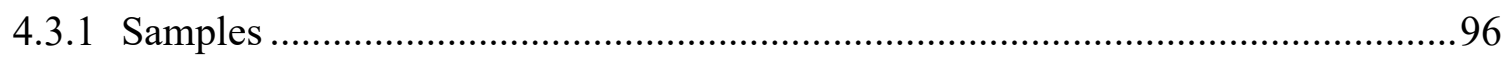

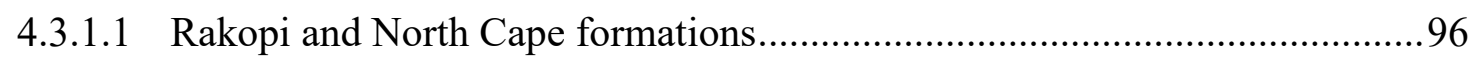

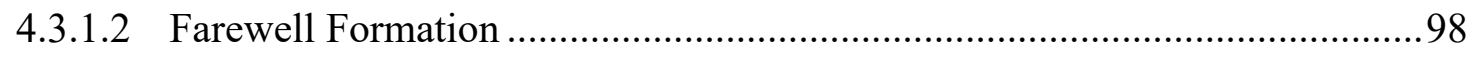

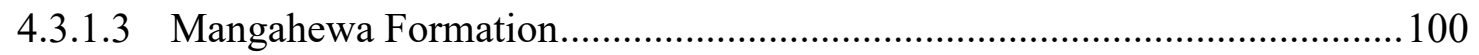

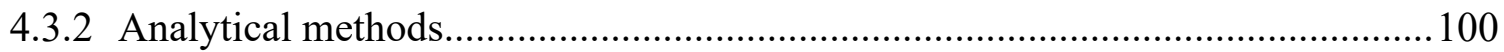

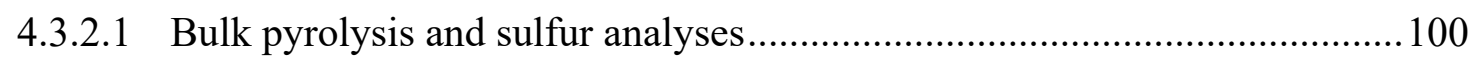

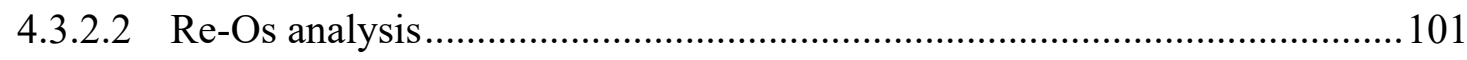

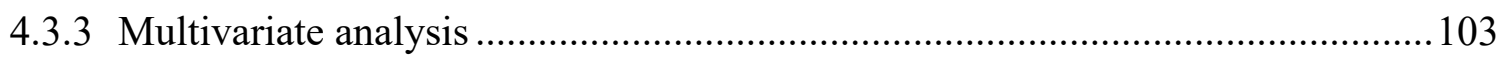

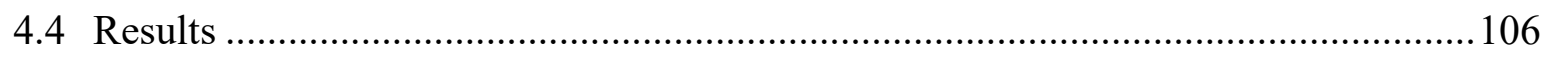

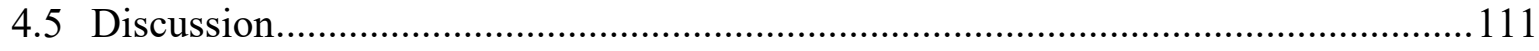

4.5.1 Sources of Os in Taranaki coals and coaly mudstones ..................................... 111

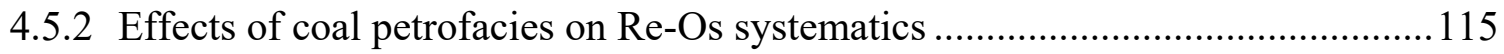

4.5.3 Effects of marine influence on Re-Os systematics of fluvio-deltaic sediments... 116

4.5.4 Re-Os geochronology in fluvio-deltaic coals and coaly mudstones ..................... 119

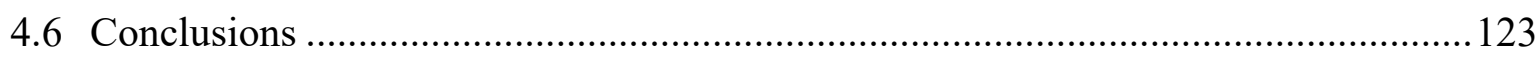

5 Re-Os systematics and Os isotope fingerprinting in crude oils sourced from terrestrial coals and marine shales, New Zealand................................... 125

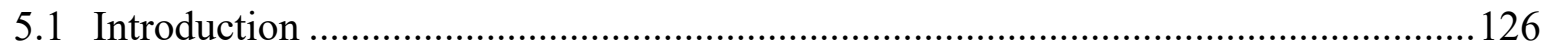

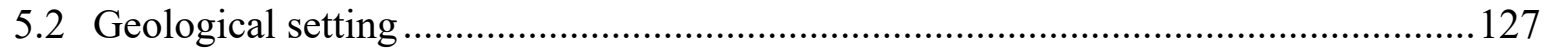

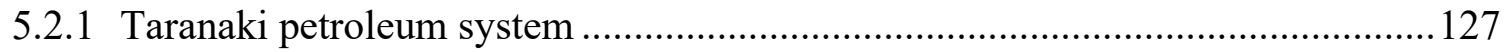

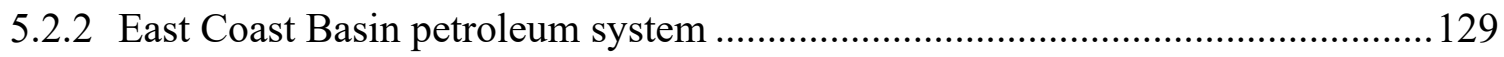

viii 


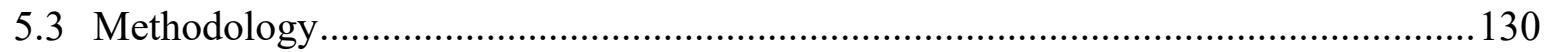

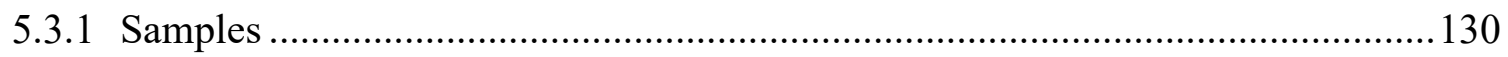

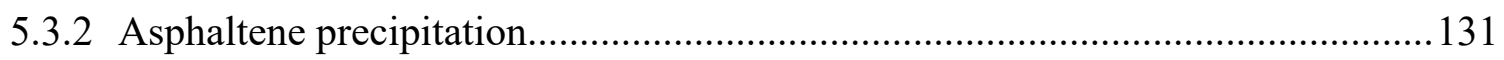

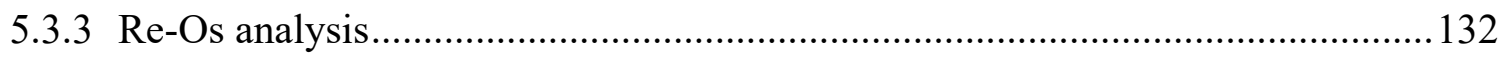

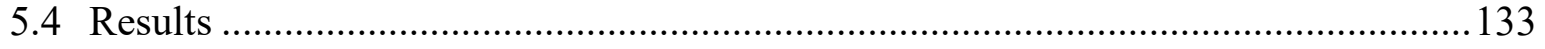

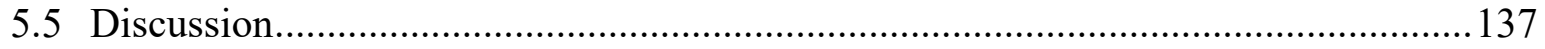

5.5.1 Re and Os systematics of coaly-sourced Family 11 oils ........................................137

5.5.2 Do the Os isotopes of Family 11 oils reflect those of potential source rocks? ....139

5.5.3 Re-Os systematics of marine Families 41 and 42 oils ......................................... 142

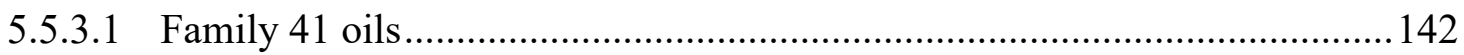

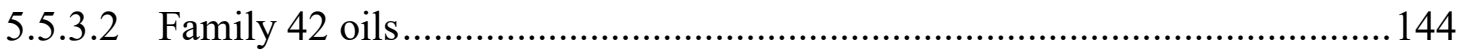

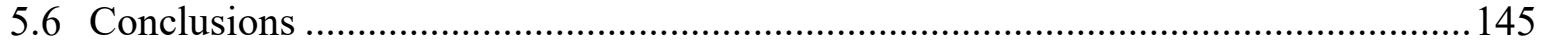

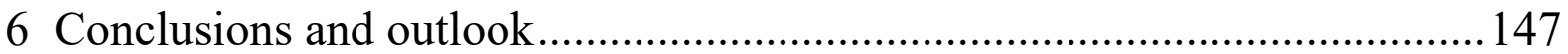

6.1 Re-Os systematics in terrestrial organic matter-dominated sediments........................147

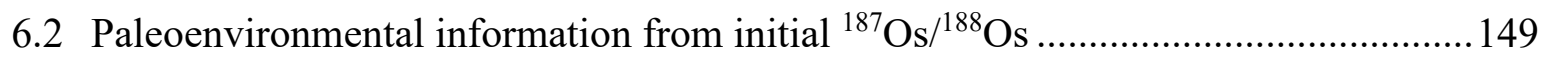

6.3 Re-Os geochronology of terrestrial organic matter-dominated sediments ..................150

6.4 Re and Os in oils generated from terrestrial organic matter-dominated sediments....152

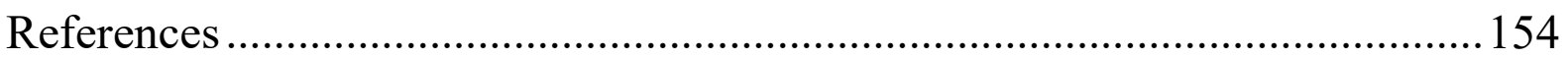

Appendix A: Geographic distribution of New Zealand oil families ................ 175

Appendix B: Rhenium test method and results .............................................. 177

B.1 Re test method - VUW Geochemistry Laboratory ......................................................177

B.1.1 Acid dissolution of rock samples ....................................................................177

B.1.2 Anion exchange chromatography ……………………………………………....177

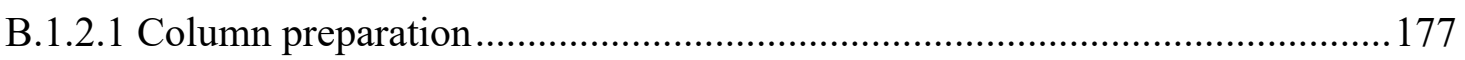

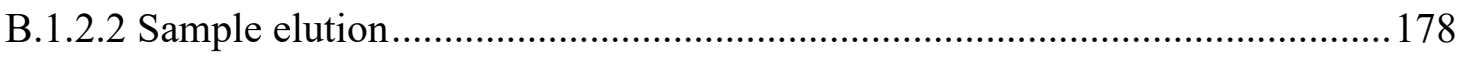

B.1.3 Isotope dilution-ICP-MS.......................................................................... 178

B.1.4 Results: Comparison of Re concentrations from Re test and full analysis ............178

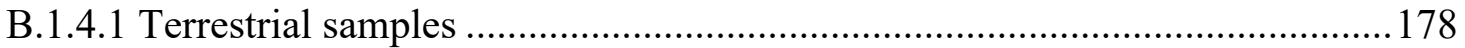

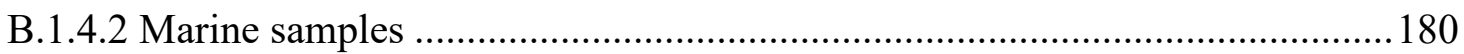




\section{List of figures}

Fig. 1.1. An illustration of the Re-Os isochron diagram showing isotopic development of four samples in time

Fig. 1.2. A schematic diagram illustrating the evolution of ${ }^{187} \mathrm{Os} /{ }^{188} \mathrm{Os}$ values in the crust and mantle from a melting event at time $\mathrm{T}$

Fig. 1.3. The first Re-Os geochronology of organic-rich sedimentary rocks

Fig. 1.4. Schematic diagram showing estimated ${ }^{187} \mathrm{Os} /{ }^{188} \mathrm{Os}$ isotope compositions, Os concentrations and Os flux for the four main sources of Os to seawater

Fig. 1.5. Marine ${ }^{187} \mathrm{Os} /{ }^{188} \mathrm{Os}$ record of the past $80 \mathrm{Ma}$ .13

Fig. 1.6. A map showing sedimentary basins of New Zealand

Fig. 1.7. A generalized chronostratigraphic panel of the Taranaki Basin across a northwestsoutheast transect

Fig. 1.8. A generalized chronostratigraphic panel of the East Coast Basin across a northwestsoutheast transect

Fig. 2.1. Flowchart summarising the Re-Os methodology

Fig. 2.2. Photographs of core materials and outcrops that were available for this project......31

Fig. 2.3. Computerized tomography images and corresponding photographs for three intervals from a drill core, showing the importance of carefully selecting samples for Re-Os geochronology.

Fig. 2.4. Photographs showing examples of terrestrial and marine oils from the Taranaki Basin and the asphaltene separation step using $n$-heptane

Fig. 2.5. Schematic diagram of a Carius tube employed in this thesis with a photograph of a metallic safety jacket used for containing sealed the Carius tube during digestion

Fig. 2.6. A photograph showing the transfer of asphaltene into Carius tube using a modified funnel and drying of the transferred asphaltene on a hot plate.

Fig. 2.7. Schematic illustration of Os micro-distillation .41

Fig. 2.8. Principles of isotope dilution as shown for Re..... 
Fig. 2.9. Principles of isotope dilution as shown for Os

Fig. 2.10. Illustration of error magnification in isotope dilution analysis .46

Fig. 2.11. Schematic diagram of the Triton negative Thermal Ionisation Mass Spectrometer with a mass spectrum showing the dominant species of $\mathrm{Re}\left(\mathrm{ReO}_{4}^{-}\right)$and $\left.\mathrm{Os}_{(\mathrm{OsO}}{ }^{-}\right)$ .48

Fig. 2.12. Typical Rock-Eval pyrogram .55

Fig. 2.13. A plot of TOC versus $S 2$ showing various classifications of hydrocarbon generative potential of a source rock. .56

Fig. 2.14. Modified Van Krevelen diagram with indications for diagenetic evolutionary pathways for type I, II and III organic matter. .57

Fig. 3.1. Map of the study area showing sample locations and distribution of Waipawa organofacies in the Southwest Pacific .64

Fig. 3.2. Chronostratigraphic column of the East Coast Basin from the Late Cretaceous to early Eocene.

Fig. 3.3. Cross-plots of bulk pyrolysis data identifying the type and maturity of organic matter present in the Waipawa and Whangai formations .72

Fig. 3.4. Cross-plots of TOC vs Re and ${ }^{192}$ Os concentrations in samples from the Waipawa and Whangai formations. .75

Fig. 3.5. Cross-plots of total sulfur vs Re concentrations, ${ }^{192} \mathrm{Os}$ concentrations and ${ }^{187} \mathrm{Re} /{ }^{188} \mathrm{Os}$ for the Waipawa and Whangai formations samples .77

Fig. 3.6. Cross-plots of normalised forms of sulfur and Re and ${ }^{192}$ Os concentrations............78

Fig. 3.7. Re-Os geochronological results for the Waipawa Formation samples from Orui-1A core.

Fig. 3.8. Re-Os geochronological results for the Waipawa Formation samples from the Taylor White section

Fig. 3.9. A GPlates global paleogeographic reconstruction of the late Paleocene (59 Ma) and a composite ${ }^{187} \mathrm{Os} /{ }^{188} \mathrm{Os}$ record of the latest Cretaceous to early Eocene period .86

Fig. 3.10. Trends in benthic stable carbon isotope values $\left(\delta^{13} \mathrm{C}\right)$, benthic oxygen isotope values $\left(\delta^{18} \mathrm{O}\right)$ and TEX 86 values 
Fig. 4.1. Map of the Taranaki Basin showing the main structural features and locations of the studied samples and a simplified geological map of the northwest Nelson region

Fig. 4.2. Generalized chronostratigraphic panel for the Taranaki Basin across a northwestsoutheast transect

Fig. 4.3. Outcrop of thin, planar mire coal beds and intervening coaly mudstone-siltstone beds within the Rakopi Formation exposed in the upper reaches of the Paturau River.

Fig. 4.4. Core description and photographs of the sampled intervals of the Farewell Formation in Kupe South-5 and -6 , respectively.

Fig. 4.5. Core description and photographs of the sampled interval of the Mangahewa Formation in Ohanga-2 well showing coal seams 10, 11 and 12

Fig. 4.6. Cross-plots of TOC vs HI, TOC vs OI, OI vs HI, HI vs $\mathrm{T}_{\max }$, OI vs $\mathrm{T}_{\max }$, and TOC vs $\mathrm{TS}$, for coaly rocks in the sampled formations.

Fig. 4.7. Cross-plots of TOC vs Re, TOC vs ${ }^{192} \mathrm{Os}$, TS vs Re, TS vs ${ }^{192} \mathrm{Os}$, Re vs ${ }^{192} \mathrm{Os}$ and $\mathrm{Re} / \mathrm{TOC}$ vs ${ }^{192} \mathrm{Os} / \mathrm{TOC}$ for coaly rocks in the sampled formations 109

Fig. 4.8. Sample scores and parameter loadings on principal components 1 and 2 110

Fig. 4.9. Initial ${ }^{187} \mathrm{Os} /{ }^{188}$ Os composition plotted against $1 / \mathrm{Os}$ and on the seawater Os curve for samples from the Mangahewa, Farewell, North Cape and Rakopi formations 113

Fig. 4.10. Paleogeographic reconstructions of the Taranaki Basin during the deposition of the Mangahewa, Farewell, North Cape and Rakopi formations 114

Fig. 4.11. Re-Os isochron diagram for the Farewell samples from Kupe South-5 and $-6 \ldots .120$

Fig. 4.12. Re-Os isochron diagram and Monte Carlo simulation of uncertainties for Mangahewa Formation samples

Fig. 5.1. Map of the study area showing locations of the studied oils in the Taranaki and East Coast basins .128

Fig. 5.2. Re and Os concentrations in the asphaltene fractions of Families 11, 41 and 42 oils and their potential source rocks from the Taranaki and East Coast basins 135

Fig. 5.3. ${ }^{187} \mathrm{Re} /{ }^{188} \mathrm{Os}$ versus ${ }^{187} \mathrm{Os} /{ }^{188} \mathrm{Os}$ for the asphaltene fractions of Family 11 oils ..... 136

Fig. 5.4. A comparison of ${ }^{187} \mathrm{Os} /{ }^{188} \mathrm{Os}$ at the time of oil generation 141 


\section{List of tables}

Table 3.1. Bulk pyrolysis and sulfur data for samples from the Waipawa and Whangai formations.

Table 3.2. Re-Os abundance and isotope data for samples from the Waipawa and Whangai formations.

Table 4.1. Re and Os concentrations and isotope compositions, sulfur and bulk pyrolysis data for samples from the Rakopi, North Cape and Farewell formations.

Table 4.2. Re and Os concentrations and isotope compositions, sulfur and bulk pyrolysis data for samples from the Mangahewa Formation 100

Table 5.1. Re and Os concentrations and isotope data for New Zealand oils 129 


\section{INTRODUCTION}

Organic-rich sedimentary rocks (total organic carbon $[\mathrm{TOC}] \geq 0.5 \mathrm{wt} \%$ ) are important and widespread components of the geologic record. These rocks typically form when specific depositional conditions that lead to enhanced preservation of organic matter such as anoxia (low oxygenation of the water column) and high sedimentation rates prevail. These conditions can occur in both marine and terrestrial (lacustrine and fluvio-deltaic) environments and may have been much more prevalent at different points in the Earth's history than at present. Organic-rich sediments, therefore, provide valuable insights into global paleoenvironmental reconstructions as well as forming direct economic resources (coal, oil and gas).

Organophilic (having a strong affinity for organic compounds) and redox-sensitive elements can be enriched in these deposits, depending on variations in depositional conditions and environmental inputs. Rhenium and Os are organophilic and further represent a parentdaughter radiogenic isotope system that can potentially be used for isotopic tracing and geochronology.

\subsection{Aims of the thesis}

The work presented in this thesis seeks to investigate factors controlling Re-Os systematics and geochronology in a range of late Cretaceous to Eocene terrestrial (fluvio-deltaic) and marine organic-rich sedimentary rocks, and their associated oils from New Zealand. This was achieved through three separate but related specific objectives:

1. Investigate how the dominance of terrestrial organic matter and high sedimentation rates during the deposition of the marine Waipawa Formation may have affected its ReOs systematics and potential for geochronology, with a further aim of establishing the first radiometric age for this formation.

2. Explore factors controlling Re-Os isotope systematics in fluvio-deltaic coals and coaly mudstones from the Taranaki Basin, in particular, the influence of syn-sedimentary marine transgressions and whether such deposits are amenable to the Re-Os geochronometer.

3. Determine whether the Re-Os isotope system can provide oil generation dates and source rock fingerprinting for New Zealand terrestrial (coaly-sourced) and marine oils. 
To accomplish these objectives, a collection of fluvio-deltaic and marine sedimentary rocks and associated oils from the Taranaki and East Coast basins were obtained. These samples are well characterized geochemically, with known age, thermal maturity and depositional environment. The samples were deposited in a range of depositional settings including distal (floodplain lake) and proximal (next to an active channel) fluvial systems, coastal plains (deltaic), shallow and deep marine systems.

This introductory chapter briefly reviews the current understanding of the Re-Os isotope system and its application to organic-rich sedimentary rocks and hydrocarbons from various depositional settings and sedimentary basins around the world, focusing particularly on examples that are of most relevance to the dominantly terrestrial (i.e., coaly) petroleum systems of New Zealand.

\subsection{The Re-Os isotope system}

In nature, there are two isotopes of Re, ${ }^{185} \mathrm{Re}$ which is stable (abundance of $37.4 \%$ ) and ${ }^{187} \mathrm{Re}$ which is radioactive (abundance of $62.6 \%$ ), decaying to a minor isotope of osmium $\left({ }^{187} \mathrm{Os}\right)$. Osmium has seven naturally occurring isotopes $\left({ }^{184} \mathrm{Os},{ }^{186} \mathrm{Os},{ }^{187} \mathrm{Os},{ }^{188} \mathrm{Os},{ }^{189} \mathrm{Os},{ }^{190} \mathrm{Os}\right.$, and ${ }^{192} \mathrm{Os}$ ), of which ${ }^{186} \mathrm{Os}$ and ${ }^{187} \mathrm{Os}$ are radiogenic. The ${ }^{186} \mathrm{Os}$ isotope is the product of alpha $(\alpha)$ decay of ${ }^{190} \mathrm{Pt}$ (Walker et al., 1997) whereas the ${ }^{187}$ Os isotope is the product of beta $\left(\beta^{-}\right)$decay of ${ }^{187} \operatorname{Re}$ (Naldrett and Libby, 1948). It is the discovery of the latter in the late 1940s that led to the initial development of the Re-Os isotope system as a geochemical tool. Research in the early decades after this discovery focused primarily on determining the half-life and decay constant of ${ }^{187} \operatorname{Re}$ (Herr et al., 1954; Hirt et al., 1963; Lindner et al., 1989; Shen et al., 1996), as well as developing analytical techniques and methodologies for precise and accurate isotopic measurement of Re and Os. To date. the most precise ${ }^{187}$ Re decay constant of $1.666 \pm 0.0017$ $\mathrm{x} 10^{-11} \mathrm{y}^{-1}$ (half-life $=41.6$ billion years) was reported by Smoliar et al. (1996) based on a study of iron meteorites. This decay constant has been confirmed by Selby et al. (2007b) through a combination of Re-Os and U-Pb zircon geochronology of magmatic ore deposits and related rocks, increasing confidence in the reliability of the Re-Os isotope system for geochronological applications.

The long half-life of ${ }^{187} \mathrm{Re}$ makes the Re-Os isotope system applicable to radiometric dating of rock materials over the length of the Earth's history (e.g., Yang et al., 2009; Rooney et al., 
2011; Simmonds et al., 2019; Washburn et al., 2019). This is achieved through the isochron method of dating where the ratio of the daughter nuclide $\left({ }^{187} \mathrm{Os}\right)$ to another non-radiogenic nuclide of the same element ( $\left.{ }^{188} \mathrm{Os}\right)$ is compared on an $\mathrm{x}-\mathrm{y}$ plot to the ratio of the parent nuclide $\left({ }^{187} \mathrm{Re}\right)$ to the same non-radiogenic nuclide $\left({ }^{188} \mathrm{Os}\right)$ of the daughter element (Fig. 1.1). At time zero, co-genetic rock samples contain different proportions of the parent isotope ratios ${ }^{187} \mathrm{Re} /{ }^{188} \mathrm{Os}$, but uniform daughter isotope ratios $\left({ }^{187} \mathrm{Os} /{ }^{188} \mathrm{Os}\right)$. With increasing time and radioactive decay of ${ }^{187} \mathrm{Re}$, the number of the ${ }^{187} \mathrm{Re}$ atoms decreases while the number of the ${ }^{187}$ Os atoms increases accordingly. The abundance of the non-radiogenic ${ }^{188}$ Os nuclide remains constant. Therefore, the ${ }^{187} \mathrm{Re} /{ }^{188} \mathrm{Os}$ ratio decreases with time as the ${ }^{187} \mathrm{Os} /{ }^{188} \mathrm{Os}$ ratio increases, which, for co-genetic samples, forms a line (isochron) that moves in a counterclockwise direction around a point intersecting the y-axis (Fig. 1.1). This point represents the initial ${ }^{187} \mathrm{Os} /{ }^{188} \mathrm{Os}(\mathrm{Os} \mathrm{i})$ of the samples. The slope of this isochron defines the age of the rock (see Section 2.7).

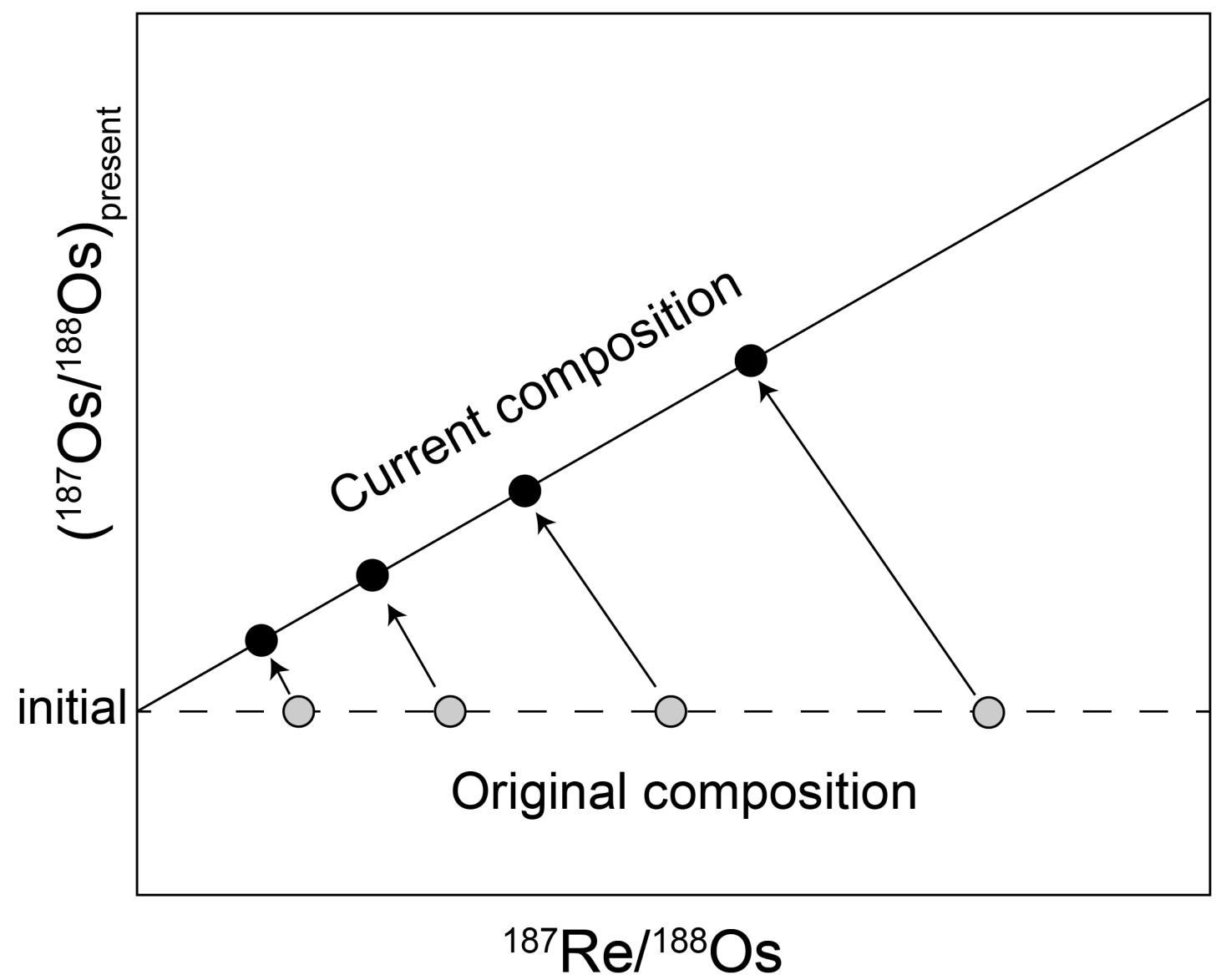

Fig. 1.1. An illustration of the Re-Os isochron diagram showing isotopic development of four samples in time. The samples had the same initial Os isotope composition at the time of formation, which then increases in a negative trajectory (shown by the arrows) as ${ }^{187} \mathrm{Re}$ decays to ${ }^{187} \mathrm{Os}$. 
Rhenium and Os are siderophile and chalcophile elements, meaning they are partitioned preferentially into metallic iron and sulfide portions of the Earth relative to silicate phases (Shirey and Walker, 1998; Dauphas et al., 2002; Barnes and Ripley, 2016; Day et al., 2016). These elements were, thus, strongly partitioned into the core during primordial differentiation of the Earth, resulting in their generally low concentrations in both the mantle and crust (Shirey and Walker, 1998; Dauphas et al., 2002). Within the crust, their chalcophilic nature leads to Re and Os being relatively concentrated in sulfide ore deposits (Shirey and Walker, 1998).

A key feature of the Re-Os isotope system that makes it useful in various geochemical applications is the difference in the compatibility of Re and Os in solid-melt systems. During mantle melting, Os is highly compatible and remains in the mantle (where it is mainly incorporated into sulfides). Rhenium is moderately incompatible and gets partitioned into the melt, becoming enriched in the Earth's crust (Fig. 1.2; Allègre and Luck, 1980; Walker et al., 1989; Righter et al., 1998; Burton et al., 2002; Harvey et al., 2016). The continental crust, therefore, exhibits high Re/Os ratios compared to the mantle which, with time, results in older crustal rocks also having ${ }^{187} \mathrm{Os} /{ }^{188} \mathrm{Os}$ ratios that are significantly higher (present-day upper continental crust [UCC] average value of 1.4) than those of the mantle $\left({ }^{187} \mathrm{Os} /{ }^{188} \mathrm{Os}=0.13\right.$; Fig. 1.2). Thus, the ${ }^{187} \mathrm{Os} /{ }^{188} \mathrm{Os}$ ratio provides a sensitive tracer of relative proportions of crustal and mantle inputs into seawater and sedimentary rocks.

These unique properties of the Re-Os isotope system have found a multitude of applications that differ from, or complement, those of other more commonly applied lithophile element radiogenic dating systems (e.g., $\mathrm{Rb}-\mathrm{Sr}, \mathrm{Sm}-\mathrm{Nd}, \mathrm{K}-\mathrm{Ar}, \mathrm{U}-\mathrm{Pb}$ ). These applications include direct determination of the timing of ore mineralisation (e.g., Herr et al., 1967; Luck and Allègre, 1982; Stein et al., 2001; Rice et al., 2016), understanding mantle evolution events and the Earth's late accretionary history (Hertogen et al., 1980; Morgan, 1986; Walker, 1988; Shirey and Walker, 1998), tracking paleoclimatic evolution (e.g., Turekian and Pegram, 1997; Ravizza et al., 2001; Ravizza and Peucker-Ehrenbrink, 2003b; Dickson et al., 2015; Rooney et al., 2016) and establishing emplacement time of large igneous provinces and other magmatic activities (e.g., Ravizza and Peucker-Ehrenbrink, 2003a; Cohen and Coe, 2007; Turgeon and Creaser, 2008; Du Vivier et al., 2014). 


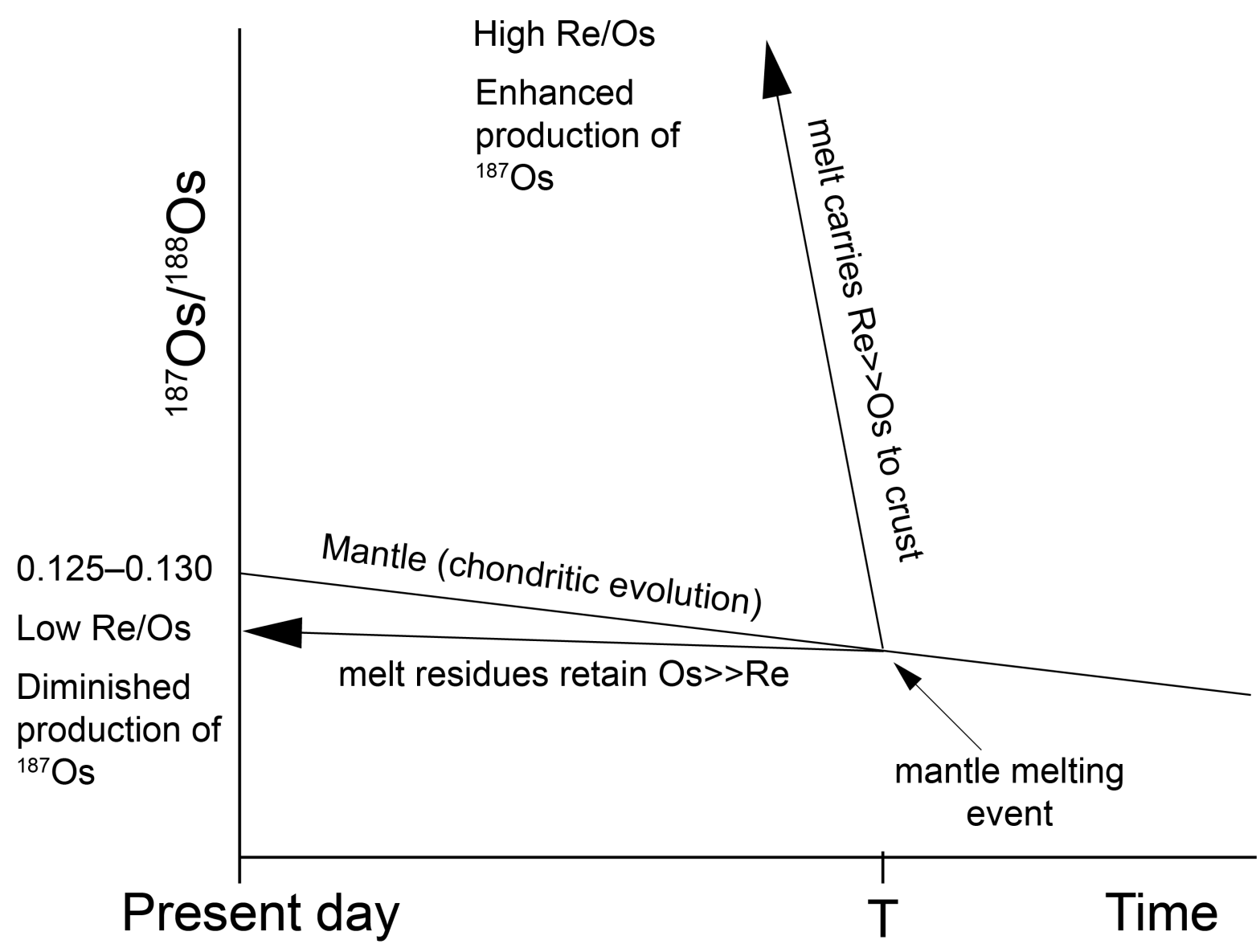

Fig. 1.2. A schematic diagram illustrating the evolution of ${ }^{187} \mathrm{Os} /{ }^{188} \mathrm{Os}$ values in the crust and mantle from a melting event at time T. Modified after Correia et al. (2007).

In addition to having siderophilic and chalcophilic properties, Re and Os are also organophilic and thus, can be enriched in sedimentary rocks rich in organic matter (Creaser et al., 2002; Selby and Creaser, 2003; Stein and Hannah, 2014). The resulting combination of high and variable Re and Os concentrations with time-dependent variations in Os isotopic composition produced by the radioactive decay of ${ }^{187} \mathrm{Re}$ to ${ }^{187} \mathrm{Os}$ allows for direct radiometric dating of the organic-rich sedimentary rocks (e.g., Cohen et al., 1999; Creaser et al., 2002; Kendall et al., 2009b; Selby et al., 2009; Rooney et al., 2010; Cumming et al., 2012; Bertoni et al., 2014; Tripathy et al., 2018; Washburn et al., 2019), provided the basic requirements for development of isochrons are met (Section 1.3.2). The application of the Re-Os isotope system has also been extended to oils, potentially providing timing constraints for generation and expulsion of oils as well as fingerprinting of oils to their potential source rocks (Selby and Creaser, 2005a; Selby et al., 2007a; Lillis and Selby, 2013; Cumming et al., 2014; Georgiev et al., 2016; Liu et al., 2018; Scarlett et al., 2019). Challenges exist, however, especially in the understanding of processes that lead to homogenisation of Os isotopes in oils and the effects of water washing 
and other secondary alteration processes in the reservoir (Mahdaoui et al., 2013; 2015; Hurtig et al., 2019; J. Liu et al., 2019).

\subsection{Re-Os systematics and geochronology in organic-rich sedimentary rocks}

\subsubsection{Re and Os sequestration in organic-rich sedimentary rocks}

Although Re and Os are present at very low overall concentrations in the Earth's crust $(\sim 0.2$ ng/g and $\sim 30$ pg/g, respectively; Peucker-Ehrenbrink and Jahn, 2001; Rudnick and Gao, 2003; Sun et al., 2003), concentrations in organic-rich sedimentary rocks are commonly much higher, with reported values of up to $470 \mathrm{ng} / \mathrm{g}$ and $4400 \mathrm{pg} / \mathrm{g}$, respectively (e.g., Ravizza and Turekian, 1989; Cohen et al., 1999; Dubin and Peucker-Ehrenbrink, 2015). The mechanisms by which these elements are sequestered into the organic-rich sediments are, however, not well understood. Studies of Re and Os enrichments in marine and lacustrine organic-rich sediments suggest that the complexation of these elements is driven by a combination of adsorption and reductive capture onto organic complexes within the water column (e.g., Colodner et al., 1993; Crusius and Thomson, 2000). However, a direct relationship between total organic carbon (TOC) and Re and Os concentrations is not always apparent as some sediments with high TOC have very low concentrations of Re and Os, and vice versa (Cohen et al., 1999; Kendall et al., 2004; Rooney et al., 2010; Selby et al., 2009). Therefore, other factors must play a role in the sequestration of the elements.

Several studies have reported enhanced uptake of Re and Os in hypoxic-anoxic water columns (Colodner et al., 1993; Crusius et al., 1996; Morford and Emerson, 1999; Crusius and Thomson, 2000; Yamashita et al., 2007; Georgiev et al., 2011), suggesting that redox conditions of the water column may play a role in Re and Os uptake into organic-rich sediments. These studies also provide evidence that Re and Os behave differently in the water column and that the uptake mechanisms may not be the same for both elements. Rhenium is believed to be sequestered by a process known as reductive capture, where $\mathrm{Re}^{\mathrm{VII}} \mathrm{O}_{4}^{-}$, the main species of Re in seawater, is reduced to insoluble $\mathrm{Re}^{\mathrm{IV}}$ under low redox potential (Eh; Colodner et al., 1993). It is also suggested that highly reducing conditions favour the removal of Re over Os from seawater (Yamashita et al., 2007). The removal of Os from the seawater occurs over a range of Eh and $\mathrm{pH}$ conditions, is directly associated with organic matter, and is relatively 
fast compared to Re removal (Poirier, 2006; Yamashita et al., 2007). It occurs first as $\mathrm{Os}^{4+}$, which is then further reduced by organic complexation to $\mathrm{Os}^{3+}$ (Yamashita et al., 2007; Kendall et al., 2009b). However, other studies have found no direct link between Re and Os uptake in organic-rich sediments and redox conditions of the depositional environment (Selby et al., 2009; Harris et al., 2013). For example, an interval of the organic-rich Woodford Shale in the USA is significantly enriched in both Re and Os (up to $\sim 40 \mathrm{ppb}$ and $4450 \mathrm{ppt}$, respectively) despite deposition in an oxic environment (Harris et al., 2013). Chapters three and four of this thesis investigate further the role of depositional redox conditions on Re and Os enrichment in organic-rich sediments.

A slow sedimentation rate has also been suggested to enhance Re and Os uptake in organicrich sediments (Crusius and Thomson, 2000; Selby et al., 2009; Cumming et al., 2012; Rooney et al., 2012). Slow sedimentation increases the time of exposure of organic matter at the sediment-water interface, allowing for more of the elements to be sequestered. However, a slow sedimentation rate may also negatively affect preservation of organic matter due to the longer residence time for oxidation (Müller and Suess, 1979; Ibach, 1982). The Waipawa Formation studied in chapter three of this thesis was deposited under high sedimentation rates $(\sim 10.6 \mathrm{~cm} / \mathrm{ky})$, which allows for an evaluation of how Re and Os enrichment in this formation compares to other formations with similar characteristics but much lower sedimentation rates.

The abundance of Re and Os in the water column, which is linked to the degree of restriction of a water body, is also considered to affect enrichment of Re and Os in organic-rich sediments (Turgeon et al., 2007; McArthur et al., 2008). In severely restricted basins where there is no replenishment of elements from the open ocean, organic matter sedimentation draws down and exhausts most of the available Re and Os in the water column (McArthur et al., 2008). Sediments that are subsequently deposited would, therefore, exhibit lower concentrations of Re and Os.

Organisms such as macroalgae that are components of sedimentary organic matter may also play a role in the uptake of Re and Os in organic-rich sediments as they have been shown to accumulate Re (up to several hundreds of ppb) and Os (tens of ppt; Racionero-Gómez et al., 2016; 2017; Ownsworth et al., 2019; Sproson et al., 2020). These organisms may release the accumulated elements back to the water column upon degradation (Racionero-Gómez et al., 2016). Therefore, only in depositional conditions that enhance preservation of organic matter 
can these organisms play a direct role in enrichment of Re and Os in organic-rich sediments, linking this mechanism to the degree of anoxia and sedimentation rate. Additional factors that may also lead to complex effects on $\mathrm{Re}$ and Os uptake in organic-rich sedimentary rocks include salinity, pH and temperature (Martin et al., 2001; Georgiev et al., 2011).

\subsubsection{Re-Os geochronology of organic-rich sedimentary rocks}

Several criteria must be met in order to obtain accurate and precise Re-Os depositional ages of organic-rich sedimentary rocks using the isochron method (e.g., Kendall et al., 2004; 2009b; Selby et al., 2009). These include: 1) the Re and Os measured in the samples are entirely hydrogenous in origin, 2) the samples are coeval and have the same Osi values, 3) immobilisation of Re and Os occurs immediately after deposition, such that the obtained ReOs ages reflect depositional and not younger, diagenetic ages, 4) the samples remain in a closed system with negligible post-depositional disturbance of the elements through processes such as weathering and contact metamorphism, and 5) there is sufficient spread in the ${ }^{187} \mathrm{Re} /{ }^{188} \mathrm{Os}$ ratios to form a well-defined isochron line.

The application of the Re-Os isotope system specifically to organic-rich sedimentary rocks was first demonstrated by Ravizza and Turekian (1989) on marine black shales of the Mississippian-Devonian Bakken Shale in North America, yielding an isochron age of $354 \pm 49$ Ma (Fig. 1.3). This age, although imprecise, is consistent (within uncertainties) with other chronological constraints for the formation. This work provided the foundation for focused research aimed at improving the accuracy and precision of Re-Os geochronology of organicrich sedimentary rocks. As analytical techniques improved, a study on Jurassic mudstones from coastal outcrops in England yielded relatively precise Re-Os ages that were within uncertainty of depositional ages interpolated from published geological timescales (Cohen et al., 1999). This study also provided the first estimates of Jurassic seawater ${ }^{187} \mathrm{Os} /{ }^{188} \mathrm{Os}$, which varied from relatively non-radiogenic values $(\sim 0.15-0.3)$ at the start of Jurassic to radiogenic values of $\sim 0.8$ in the Toarcian followed by a decrease to $\sim 0.6$ towards the end of the Jurassic. These changes in ${ }^{187} \mathrm{Os} /{ }^{188}$ Os were attributed to variable contributions of Os to the seawater from weathering of older continental strata versus volcanic and hydrothermal activities (Cohen et al., 1999). 


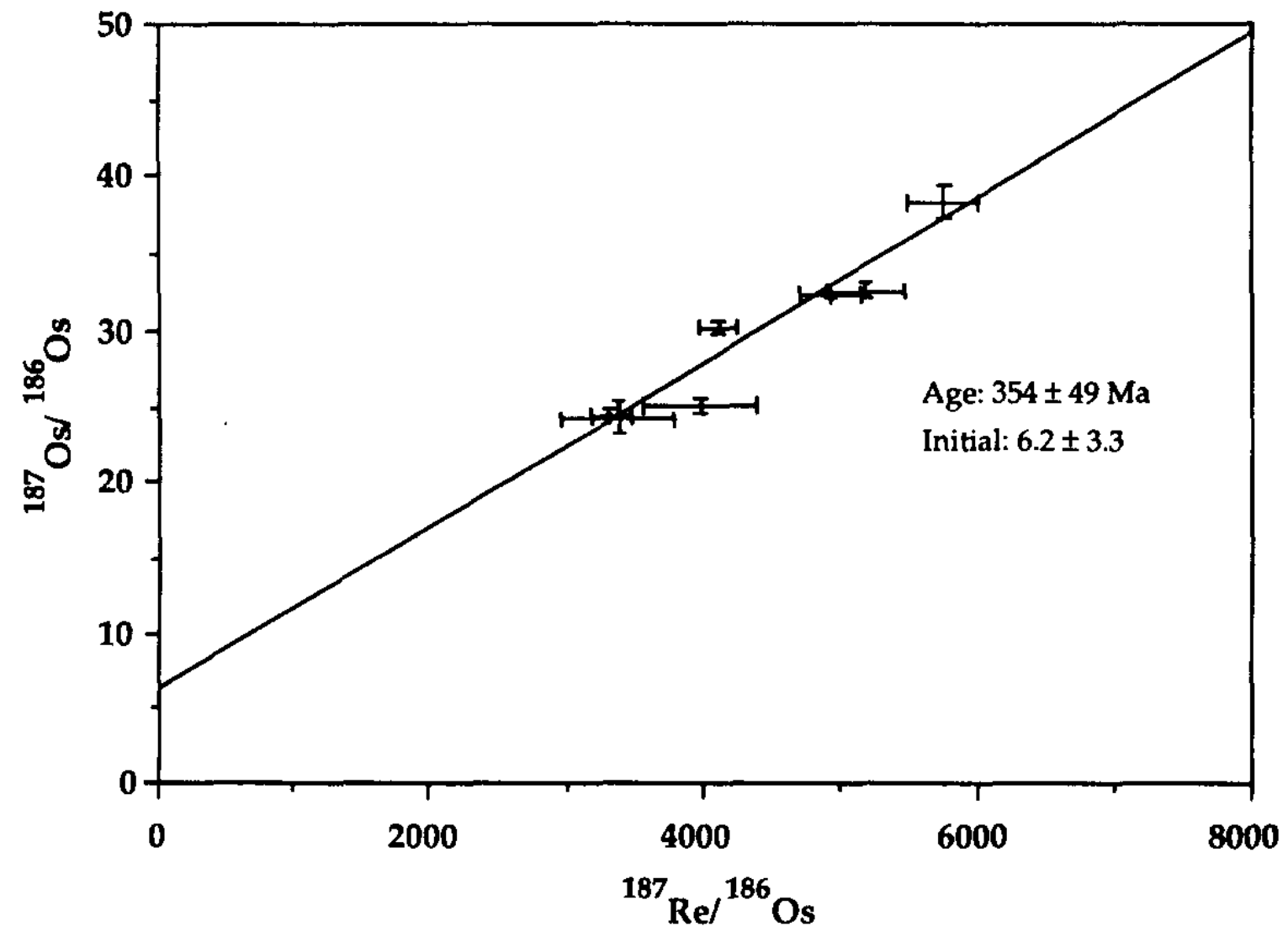

Fig. 1.3. The first Re-Os geochronology of organic-rich sedimentary rocks, performed on the Mississippian-Devonian Bakken Shale Formation, yielding an age $354 \pm 49 \mathrm{Ma}$ (Ravizza and Turekian, 1989).

The last two decades have seen a tremendous increase in the number and complexity of organic-rich sedimentary rocks that have been accurately and precisely (in some cases, $<1 \%$ uncertainty, 2 $\sigma$ ) dated using the Re-Os geochronometer (e.g., Creaser et al., 2002; Kendall et al., 2004; Selby et al., 2009; Rooney et al., 2010; Cumming et al., 2012; Georgiev et al., 2017; Tripathy et al., 2018; van Acken et al., 2019). A study on the Late Devonian Exshaw Formation, Canada, not only provided an accurate and relatively precise Re-Os age of $358 \pm 10$ Ma but also revealed that thermal maturation of organic-rich sediments and petroleum migration does not significantly affect Re-Os systematics in organic-rich sediments (Creaser et al., 2002). These findings have been corroborated through hydrous pyrolysis experiments, which show that minimal proportions of $\mathrm{Re}$ and Os (about 5\%) are transferred from sedimentary rocks to generated hydrocarbons (Rooney et al., 2012). A study on the Neoproterozoic Old Fort Point Formation, Canada, further demonstrated that accurate and precise Re-Os depositional ages can be obtained from sedimentary rocks that have low TOC content $(<1 \mathrm{wt} \%)$ and have experienced low-grade contact metamorphism (Kendall et al., 2004). Similarly, Rooney et al. 
(2011) successfully obtained a precise Re-Os depositional age (659.6 $\pm 9.6 \mathrm{Ma})$ for sedimentary rocks from the Dalradian Supergroup, Scotland, that have low TOC content and have undergone anhydrous metamorphism. This study also showed for the first time that precise Re-Os geochronology is attainable on sedimentary rocks that contain very low Re and Os concentrations ( $<1 \mathrm{ppb}$ and $<50 \mathrm{ppt}$, respectively).

The first comprehensive study on Re-Os geochronology of lacustrine sediments yielded depositional ages for organic-rich intervals of the Green River Formation, USA, that are in agreement (within uncertainty) with established $\mathrm{U}-\mathrm{Pb}$ and Ar-Ar ages for these units (e.g., 49.2 $\pm 1.0 \mathrm{Ma}$ for the Douglas Creek Member; Cumming et al., 2012). These results suggested that Re-Os geochronology is not restricted to marine rocks, but that any sedimentary rock deposited in conditions that enhance the preservation of organic matter may be viable. In addition, this study provided valuable insights into factors controlling the variable uptake of Re and Os and their subsequent fractionation in organic-rich sediments, suggesting that these processes may be related to variation in depositional environment (proximal versus distal lake settings) and organic matter type (algal vs terrestrial).

Lastly, the Re-Os geochronometer has been successfully extended to marine-influenced coals, with Tripathy et al. (2015) reporting a depositional age of $325 \pm 14$ Ma for the Matewan coal bed, USA, consistent with other chronological constraints. The Matewan coals exhibit average $\operatorname{Re}(108 \mathrm{ppb})$ and Os (520 ppt) concentrations that are up to 2 orders of magnitude higher than those reported for the UCC (0.2-2 ppb Re and 30-50 ppt Os; Esser and Turekian, 1993; Peucker-Ehrenbrink and Jahn, 2001; Sun et al., 2003). This study motivated Goswami et al. (2018) to test the applicability of the Re-Os geochronometer to Carboniferous freshwater coals and shales from the Finnmark Platform (Soldogg Formation) and the Central Appalachian Basin (Fire Clay coals). The Soldogg Formation shales and coals, however, exhibit ${ }^{187} \mathrm{Re} /{ }^{188} \mathrm{Os}$ and ${ }^{187} \mathrm{Os} /{ }^{188} \mathrm{Os}$ ratios that are scattered without any discernible linear trend on an isochron diagram. This was attributed to heterogeneity of the Osi values and a lack of diagenetic isotopic equilibration (Goswami et al., 2018). The ${ }^{187} \mathrm{Re} /{ }^{188} \mathrm{Os}$ and ${ }^{187} \mathrm{Os} /{ }^{188} \mathrm{Os}$ ratios for the Fire Clay coals are well correlated on an isochron diagram but the age produced is significantly older than published $\mathrm{U}-\mathrm{Pb}$ and Ar-Ar depositional ages for a tonstein interbedded with the coals. This age discrepancy was attributed to binary mixing of Re and Os derived from two different components (coals and tonstein) with different ${ }^{187} \mathrm{Re} /{ }^{188} \mathrm{Os}$ and Osi ratios (Goswami et al., 
2018). The work presented in Chapter 4 of this thesis builds on the results of Tripathy et al. (2015) and Goswami et al. (2018), further assessing factors controlling Re-Os systematics and potential for geochronology in organic-rich fluvio-deltaic sediments.

\subsubsection{Os isotopes as a geochemical tracer}

Rhenium and Os incorporated into organic-rich sediments are believed to be hydrogenous in origin, sequestered from the water column at the time of deposition (Cohen et al., 1999; Selby and Creaser, 2003). Organic-rich sedimentary rocks can, therefore, record a vertical sequence of changing Re and Os chemistry of the aquatic environment, which in turn reflects the integrated effects of global and local processes that contribute Re and Os to the water body such as continental weathering, glaciation, volcanic activity and extra-terrestrial impacts (Ravizza et al., 2001; Paquay et al., 2008; Zaiss et al., 2014; Rooney et al., 2016; Them et al., 2017). In particular, the Osi of organic-rich sedimentary rocks records a balance of Os sources to the contemporaneous waterbody: aeolian dust $\left({ }^{187} \mathrm{Os} /{ }^{188} \mathrm{Os}=\sim 1.05\right)$ and rivers that drain older continental strata $\left({ }^{187} \mathrm{Os} /{ }^{188} \mathrm{Os}=\sim 1.4\right)$ provide a radiogenic end-member whereas hydrothermal alteration or magmatic activity $\left({ }^{187} \mathrm{Os} /{ }^{188} \mathrm{Os}=\sim 0.12\right)$ and extra-terrestrial materials (e.g., cosmic dust and bolides with ${ }^{187} \mathrm{Os} /{ }^{188} \mathrm{Os}=\sim 0.12$ ) provide a non-radiogenic end-member (Fig. 1.4; Pegram and Turekian, 1999; Peucker-Ehrenbrink and Ravizza, 2000; Cohen, 2004). Cosmic and aeolian dust contributes only a small proportion of the global Os flux and therefore, does not appreciably affect the ${ }^{187} \mathrm{Os} /{ }^{188} \mathrm{Os}$ of the water bodies (PeuckerEhrenbrink and Ravizza, 2000).

The dominant source of Os to the present-day ocean, lake and estuarine waters is weathering of the upper continental crust, although this balance has not been constant throughout the Cenozoic - the Osi of seawater in the past has been appreciably lower than the modern ${ }^{187} \mathrm{Os} /{ }^{188} \mathrm{Os}$ value of $\sim 1.09$, requiring more influence from non-radiogenic sources (e.g., values of $\sim 0.36$ around 34-36 Ma; Turekian and Pegram, 1997; Pegram and Turekian, 1999; PeuckerEhrenbrink and Ravizza, 2000). An important feature of Os as a geochemical tracer is its short residence time in the water column $\left(\sim 10^{3}-10^{5} \mathrm{yr}\right)$, which allows it to respond rapidly to transient changes in the composition of the Os influx (Cohen, 2004; Rooney et al., 2016). 


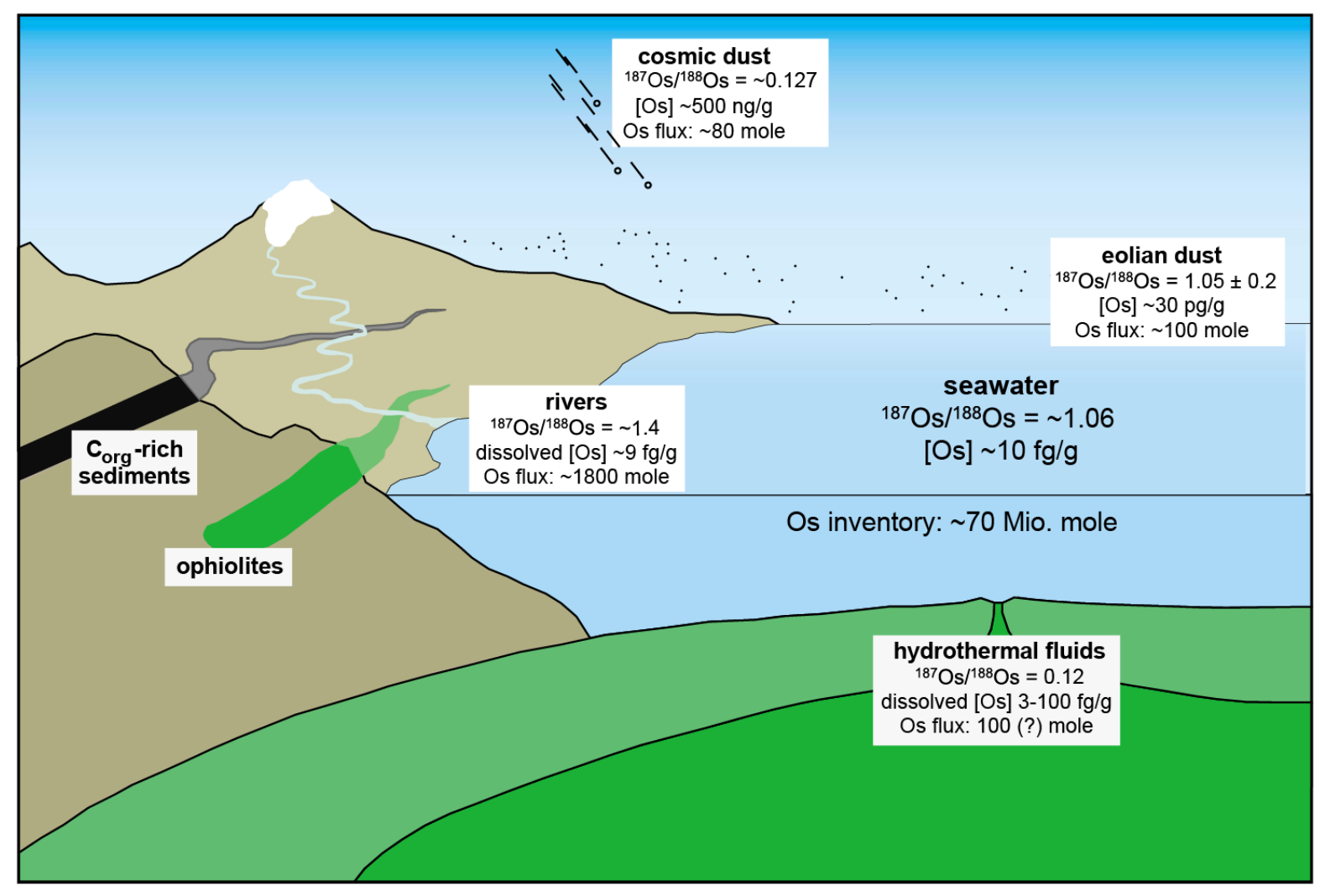

Fig. 1.4. Schematic diagram showing estimated ${ }^{187} \mathrm{Os} /{ }^{188} \mathrm{Os}$ isotope compositions, Os concentrations and Os flux for the four main sources of Os to seawater (modified after Peucker-Ehrenbrink and Ravizza, 2000).

The first synoptic record of variation in seawater Os isotope composition through the Cenozoic was obtained from a sediment core in the North Pacific comprising pelagic clay sediments (Pegram et al., 1992; Turekian and Pegram, 1997; Pegram and Turekian, 1999). These studies showed that the Cenozoic marine ${ }^{187} \mathrm{Os} /{ }^{188} \mathrm{Os}$ record (initially reported as ${ }^{187} \mathrm{Os} /{ }^{186} \mathrm{Os}$, but reporting using this ratio was abandoned after the realisation that ${ }^{186} \mathrm{Os}$ is the decay product of ${ }^{190} \mathrm{Pt}$; Walker et al., 1997) is characterized by a shift from generally non-radiogenic values of $\sim 0.23$ at the beginning of the Paleogene period to more radiogenic values of 0.99 in the Neogene. Four excursions to low ${ }^{187} \mathrm{Os} /{ }^{188} \mathrm{Os}$ are recognized in this record (Fig. 1.5): 1) at the K-Pg boundary ( $\sim 66 \mathrm{Ma}$ ) associated with the Chicxulub impact event, 2$)$ in the mid-Paleocene ( $\sim 59 \mathrm{Ma})$, cause not well understood, 3) in the late Eocene ( $35.4 \mathrm{Ma})$ associated with the Chesapeake Bay and Popigai impact events and 4) at or close to the Eocene-Oligocene boundary ( $\sim 34.5 \mathrm{Ma})$ attributed to global cooling and glaciation (Pegram and Turekian, 1999; Ravizza and Peucker-Ehrenbrink, 2003b; Dalai et al., 2006; Ravizza, 2007; Paquay et al., 2008; Zaiss et al., 2014). The work presented in chapter three of this thesis includes an assessment of 
the potential causes of the mid-Paleocene ${ }^{187} \mathrm{Os} /{ }^{188} \mathrm{Os}$ minimum, contributing new data from the New Zealand Whangai and Waipawa formations deposited during this period.

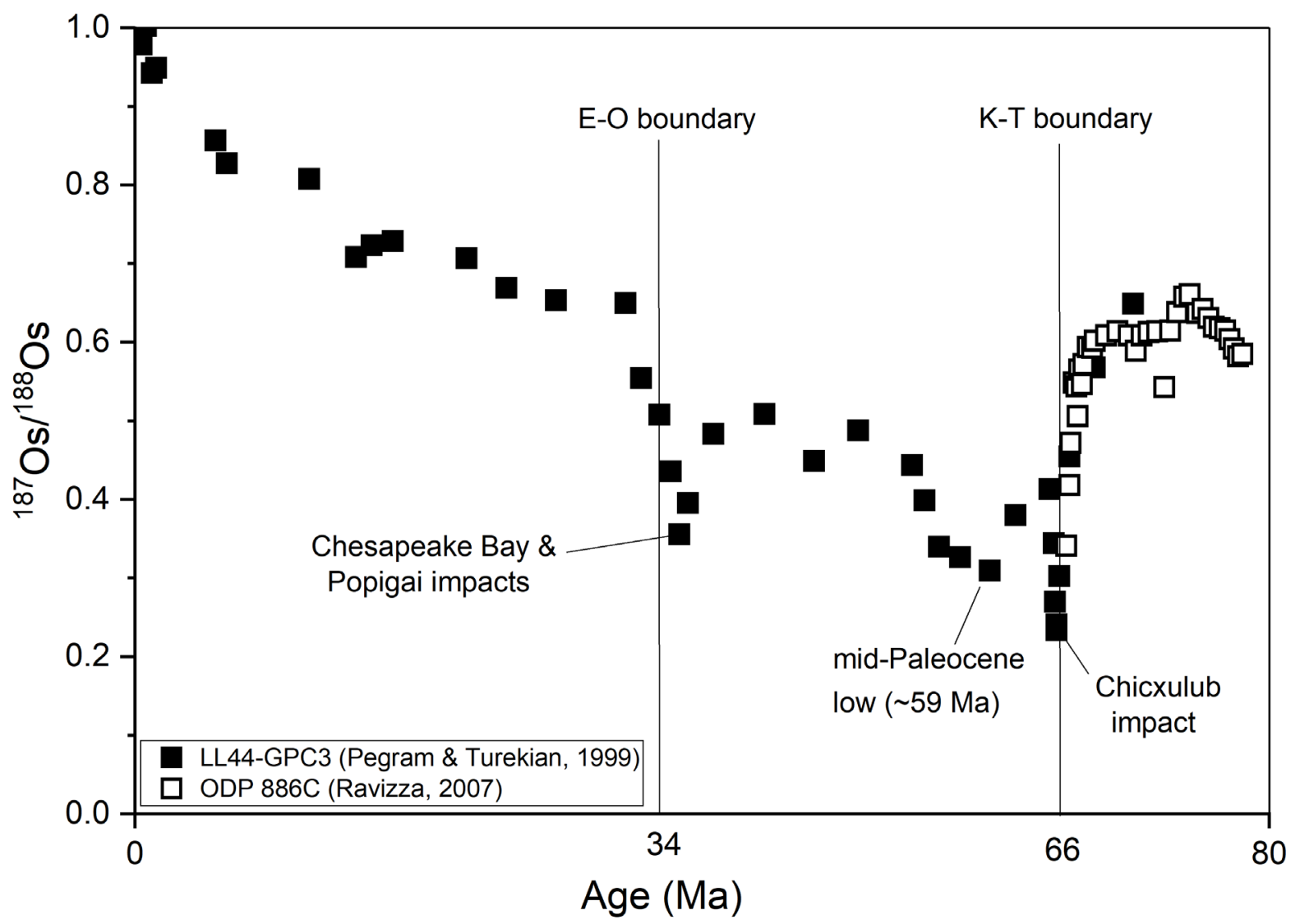

Fig. 1.5. Marine ${ }^{187} \mathrm{Os} /{ }^{188} \mathrm{Os}$ record of the past $80 \mathrm{Ma}$, reconstructed from leachable Os in pelagic clay sediments (Pegram and Turekian, 1999; Ravizza, 2007). The temporal resolution in this record is too low to differentiate between the minima caused by the late Eocene impact events and the EoceneOligocene climate transition.

Several studies have demonstrated the usefulness of the Os isotopic tracer in understanding the interplay between continental weathering, and paleoclimatic and palaeoceanographic processes. The relatively rapid shift to higher ${ }^{187} \mathrm{Os} /{ }^{188} \mathrm{Os}$ in the late Paleocene has been attributed to a global increase in continental weathering rates in response to an accelerated hydrological cycle that is linked to the Paleocene-Eocene thermal maximum (PETM; Ravizza et al., 2001; Wieczorek et al., 2013; Dickson et al., 2015; Z. Liu et al., 2019a). Glacialinterglacial cycles have also been shown to cause variations in the marine ${ }^{187} \mathrm{Os} /{ }^{188} \mathrm{Os}$ record, with less radiogenic ${ }^{187} \mathrm{Os} /{ }^{188} \mathrm{Os}$ values recorded during the height of glacial periods due to decreased delivery of radiogenic Os from the continents (Ravizza and Peucker-Ehrenbrink, 2003b; Dalai et al., 2005; Finlay et al., 2010; Paquay and Ravizza, 2012; Rooney et al., 2016). 
These variations in the marine ${ }^{187} \mathrm{Os} /{ }^{188} \mathrm{Os}$ record aid in the reconstruction of ice sheet fluctuations which in turn provide useful indications of past climatic changes (Rooney et al., 2016). Fluctuations in the marine ${ }^{187} \mathrm{Os} /{ }^{188}$ Os record have also provided insights into the effect of emplacement of large igneous provinces on marine and terrestrial biota (Cohen and Coe, 2002; 2007; Ravizza and Peucker-Ehrenbrink, 2003a; Turgeon and Creaser, 2008; Robinson et al., 2009; Tejada et al., 2009; Wieczorek et al., 2013; Du Vivier et al., 2014; 2015; Percival et al., 2016; Z. Liu et al., 2019b).

\subsection{Re-Os systematics and geochronology in petroleum systems}

Measurable quantities of $\mathrm{Re}$ and Os have long been reported in crude oil and bitumen (Poplavko et al., 1975; Barre et al., 1995; Woodland et al., 2001). Rhenium concentrations (5$200 \mathrm{ppb}$ ) were first reported in crude oils from Russia and Asia, where in some cases, light oils contained higher concentrations of Re than heavy oils (Poplavko et al., 1975). Crude oil from fields around the UK showed Re contents ranging from 0.9 to $2.1 \mathrm{ppb}$ (Woodland et al., 2001). The first Os concentrations (60-1500 ppt) and isotopic compositions $\left({ }^{187} \mathrm{Os} /{ }^{188} \mathrm{Os}=0.132-\right.$ 0.995) for crude oils were reported for oils from the Paris Basin, France (Barre et al., 1995). Since then, the number of $\mathrm{Re}$ and Os analyses in crude oil and bitumen fractions has significantly increased leading to a better understanding of the nature, distribution and fractionation of these elements in petroleum (e.g., Finlay et al., 2011; Mahdaoui et al., 2013; Cumming et al., 2014; Georgiev et al., 2016; Liu et al., 2018; Hurtig et al., 2019).

Hydrous pyrolysis experiments have shown that Re and Os are transferred from the source rock to generated crude oil and bitumen upon thermal maturation of organic matter (Rooney et al., 2012; Cumming et al., 2014). However, only 5\% of Re and Os in the source rock appear to be transferred into the oil, implying that the majority of Re and Os remains complexed within the kerogen fraction of the rock (Cumming et al., 2014). These experiments also show that the generated oils inherit ${ }^{187} \mathrm{Re} /{ }^{188} \mathrm{Os}$ and ${ }^{187} \mathrm{Os} /{ }^{188} \mathrm{Os}$ isotope compositions of the source rock, corroborating studies in natural systems that suggest that thermal maturation of organic matter does not disturb Re-Os systematics (Creaser et al., 2002; Selby and Creaser, 2003). Despite reaching peak oil generation temperature $\left(330-350{ }^{\circ} \mathrm{C}\right)$, the $\mathrm{Re}$ and Os concentrations in artificially generated oils are, however, low compared to those reported for natural crude oil (Rooney et al., 2012). This was attributed to kinetic differences between laboratory conditions 
and natural systems where in hydrous pyrolysis experiments, generation of crude oil occurs under high temperatures $\left(250-350^{\circ} \mathrm{C}\right)$ and short period of time $(72 \mathrm{~h})$. In contrast, generation of crude oil in natural petroleum systems occurs under lower temperatures of $50-150{ }^{\circ} \mathrm{C}$ and over millions of years.

\subsubsection{Residency and fractionation of Re and Os within crude oil}

The location of $\mathrm{Re}$ and Os within different crude oil fractions was first investigated in asphaltene and maltene fractions of 17 worldwide oils (Selby et al., 2007a), with $\geq 90$ and 83\% of Re and Os, respectively, found to reside in the asphaltene fraction. More recent studies have confirmed these findings (Georgiev et al., 2016; DiMarzio et al., 2018; J. Liu et al., 2019). Importantly, the ${ }^{187} \mathrm{Re} /{ }^{188} \mathrm{Os}$ and ${ }^{187} \mathrm{Os} /{ }^{188} \mathrm{Os}$ of the asphaltene fractions are in most cases comparable to those of the whole oil and can thus be used to approximate the Re-Os isotopic compositions of the whole oil (Selby et al., 2007a). Separation of the asphaltene fraction for Re-Os analyses, therefore, effectively pre-concentrates these elements and allows for more precise Re-Os data to be obtained. This is particularly important for oils that have low Re and Os concentrations, such as the coaly-sourced oils from the Taranaki Basin studied in this thesis. More recent studies have, however, revealed that for some oils: 1) differences exist in ${ }^{187} \mathrm{Re} /{ }^{188} \mathrm{Os}$ and ${ }^{187} \mathrm{Os} /{ }^{188} \mathrm{Os}$ of the whole oil and asphaltene fractions, with the latter generally having higher (up to 30\%) and more radiogenic values, respectively, and 2) the maltene fraction may hold significant $(\leq 48 \%)$ proportions of Re and Os (Georgiev et al., 2016; J. Liu et al., 2019). Using the asphaltene fraction could, therefore, result in inaccuracies in Re-Os isotopic and geochronological data if post-formation exchange between the asphaltene and maltene fractions has occurred. The asphaltene fraction, however, remains the best option in situations where precise data are required but cannot be obtained from whole oils due to very low $\operatorname{Re}(<1$ $\mathrm{ppb})$ and Os $(<50 \mathrm{ppt})$ bulk concentrations. For example, Re-Os analyses were carried out on the asphaltene fractions of oils from Duvernay petroleum system in the Western Canada sedimentary basin because the bulk oils have low Re (0.04-3.78 ppb) and Os (0.6-41.2 ppt) concentrations (Liu et al., 2018). The Re-Os dates obtained from the asphaltene fractions in this study are consistent with oil generation dates obtained from Ar-Ar geochronology and basin modelling, indicating that this method is still viable for Re-Os oil geochronology.

The fractionation of Re and Os within crude oil has been investigated through progressive precipitation of asphaltene sub-fractions (Mahdaoui et al., 2013; DiMarzio et al., 2018; J. Liu 
et al., 2019). Mahdaoui et al. (2013) found that, although Re and Os concentrations decreased with progressive precipitation of asphaltene sub-fractions, the Re/Os elemental ratios largely remained constant. This implies that natural precipitation of asphaltene in petroleum systems may have minimal effect on Re/Os ratios and thus, unlikely to disturb the Re-Os geochronometer (Mahdaoui et al., 2013). However, DiMarzio et al. (2018) showed that progressive precipitation of asphaltenes can alter the ${ }^{187} \mathrm{Re} /{ }^{188} \mathrm{O}$ isotopic ratios of crude oil depending on the solvent (precipitant) used and the amount of asphaltenes precipitated. A similar observation was made by J. Liu et al. (2019) for six oils from different geographic locations. Progressively precipitated asphaltene sub-fractions from these oils exhibited different ${ }^{187} \mathrm{Re} /{ }^{188}$ Os and ${ }^{187} \mathrm{Os} /{ }^{188} \mathrm{Os}$, which Liu et al., (2019a) suggested may be related to the nature of the oil itself, such as its origin, maturity and alteration rather than an analytical artefact resulting from the precipitation process.

Although the majority of Re and Os in crude oil is now believed to be held in the asphaltene fraction, the exact mechanism of how these elements are bound within the oil is not known. Selby et al. (2007a) suggested that Re and Os may be predominantly bound by heteroatomic ligands, including porphyrins similar to $\mathrm{Ni}$ and $\mathrm{V}$. However, more recent studies have suggested that these elements are unlikely to be bound in porphyrins because they are mainly hosted in the more polar asphaltene sub-fractions than those hosting Ni and V (DiMarzio et al., 2018; J. Liu et al., 2019). Therefore, this remains an area for future research.

\subsubsection{Re-Os geochronology of crude oil}

Re-Os geochronology of crude oil is still in its early stages, with limited studies reported to date due to the general lack of understanding of the behaviour of Re and Os in the oil. The possibility of obtaining time constraints for generation, expulsion or migration of hydrocarbons, however, remains a driver for research in this field. This is because traditional methods for dating of these events (i.e., basin modelling) often yield inaccurate dates because they rely on estimated input parameters such as heat flow, source rock properties and kinetics, paleo water depth, and different erosion scenarios (e.g., Al-Hajeri et al., 2009). Evidence of the ability of the Re-Os system to date hydrocarbons was first demonstrated on bitumen found in open-space fillings and coatings of a Polaris Mississippi Valley-type $\mathrm{Zn}-\mathrm{Pb}$ deposit (Selby et al., 2005b). This bitumen yielded a Re-Os isochron age of $374.2 \pm 8.6 \mathrm{Ma}$ that is consistent with $\mathrm{Rb}-\mathrm{Sr}$ ages from sphalerite and paleomagnetic dates for mineralization of the $\mathrm{Zn}-\mathrm{Pb}$ 
deposit. Another early Re-Os study on hydrocarbon deposits yielded an isochron date of $112 \pm$ 5.3 Ma for the timing of emplacement of the giant oil sand deposits of Alberta, Canada (Selby and Creaser, 2005a). This age ruled out previous models that had indicated that orogenic events were the main driver for generation of the oil sands and instead suggested oil generation from older source rocks.

A more comprehensive study of 18 oils with clearly defined source and generation ages from the UK Atlantic margin yielded a Re-Os age $(68 \pm 13 \mathrm{Ma})$ that is in agreement with known oil generation ages obtained from basin modelling and Ar-Ar geochronology (Finlay et al., 2011). This study provided the first clear evidence that Re-Os oil geochronology can record oil generation events rather than migration events. Lillis and Selby (2013) further examined whether Re-Os oil isochrons record the timing of generation, migration, source rock age, or post emplacement processes such as thermochemical sulfate reduction (TSR). They studied crude oils from the Permian Phosphoria Formation, Bighorn Basin, USA, which revealed two ages: 1) a precise Miocene age of $9.24 \pm 0.39$ Ma for oils that had experienced TSR and 2) a Triassic age of $211 \pm 21$ Ma for oils that had not been affected by TSR. The latter age is consistent with the early stage of oil generation in the Phosphoria petroleum system, further supporting the hypothesis that oil isochrons date oil generation events. The Miocene age was suggested to reflect the end of TSR in the reservoir as it coincides with uplift and erosion of the overburden in the last $10 \mathrm{Myr}$, which potentially lowered the temperature of the reservoir to a point where TSR was no longer viable (Lillis and Selby, 2013). Another instance where the Re-Os oil geochronometer has been affected by secondary processes in the reservoir was recently shown for low and high maturity bitumen from southern China (Kroeger et al., 2016). The low maturity bitumen yielded a Re-Os age of $\sim 430 \mathrm{Ma}$, which is consistent with the timing of initial crude oil generation as indicated by basin models and $\mathrm{Rb}$-Sr bitumen dating. In contrast, the Re-Os data for the high maturity bitumen yielded a considerably younger age of $\sim 70 \mathrm{Ma}$. This younger age coincides with an orogenic event, possibly indicating cessation of thermal cracking and generation of dry gas (Ge et al., 2016). More recently, Re-Os geochronology of a relatively simple petroleum system (Duvernay) in the Western Canada Sedimentary Basin revealed that Re-Os oil isochrons for the crude oils have not been affected by secondary alteration and indeed record oil generation events (Liu et al., 2018). 
The first application of the Re-Os oil geochronometer to petroleum sourced from lacustrine rocks (Green River Formation) yielded an oil generation age with extremely large uncertainties $(19 \pm 14 \mathrm{Ma})$ but which is broadly consistent with dates obtained from basin modelling $(\sim 25$ Ma; Cumming et al., 2014). The large uncertainty was attributed to multiple oil generation events that may have occurred within the thick $(\sim 3000 \mathrm{~m})$ Green River Formation, creating a mixture of petroleum phases with different Osi (Cumming et al., 2014). When samples were separated based on the similarity of Osi, the precision of the obtained Re-Os age significantly improved (33 $\pm 12 \mathrm{Ma}$ and $29.9 \pm 9.5 \mathrm{Ma}$ ), consistent with the interpretation of multiple oil generation episodes sourced from different parts of the Formation. Despite the large uncertainties, this study demonstrated for the first time that Re-Os oil geochronology is not necessarily limited to crude oil derived from marine petroleum systems but can also be applied to lacustrine systems. The potential of the Re-Os isotope system to date crude oils sourced from coaly rocks has not been explored. The work presented in chapter five of this thesis provides the first assessment of Re-Os systematics and geochronology in oils sourced from terrestrial coals.

The Re-Os oil geochronology studies described above are based on multiple oil (or bitumen) samples from different parts of a reservoir to obtain Re-Os data with sufficient spread in ${ }^{187} \mathrm{Re} /{ }^{188} \mathrm{Os}$. The use of multiple oils with uncertain relationships, however, makes interpretation of the Re-Os data difficult. Georgiev et al. (2016) proposed a different approach for Re-Os oil geochronology based on isotopic analyses of different fractions (asphaltenes and maltenes) within a single sample of crude oil. The ${ }^{187} \mathrm{Os} /{ }^{188} \mathrm{Os}$ and most of the ${ }^{187} \mathrm{Re} /{ }^{188} \mathrm{Os}$ ratios in the maltene fractions of three oils from the Gela oil field in Italy are lower than in the corresponding asphaltene fractions. Whole oils, on the other hand, display intermediate ${ }^{187} \mathrm{Re} /{ }^{188} \mathrm{Os}$ and ${ }^{187} \mathrm{Os} /{ }^{188} \mathrm{Os}$ ratios, largely because they are physical mixtures of the two fractions. This spread in isotope ratios provided the basis for developing an isochron, potentially eliminating the uncertainty of using multiple oils from different parts of a reservoir that may have undergone different generation, migration or reservoir modification histories (Georgiev et al., 2016). However, these results have not been reproduced by J. Liu et al. (2019) in their study of six geographically distinct oil samples, as they found that no combinations of Re-Os isotopic compositions of fractions of a single crude oil consistently yielded geologically meaningful Re-Os ages. In this thesis, sampling for geochronology followed the initial approach of obtaining geochemically similar oils from different parts of a basin. 
Although it has been demonstrated that Re-Os geochronology in crude oils can yield time constraints for oil generation events, questions remain as to the exact processes that lead to Os homogenisation in oils and the role of secondary alteration processes such as TSR and water washing. Contact experiments between natural crude oils and aqueous solutions have been undertaken to try and establish whether mixing of crude oil with formation waters in the reservoir (water washing) has the potential to overprint Re-Os systematics and reset the Re-Os geochronometer in crude oil and bitumen (Mahdaoui et al., 2015; Hurtig et al., 2019). Mahdaoui et al. (2015) demonstrated that Re and Os can transfer significantly (up to 97\%) and rapidly (within a few days) from the aqueous phase into oils, regardless of temperature, oil composition or element concentration. Hurtig et al. (2019) corroborated these findings and further showed that oil-water interaction is capable of resetting the Re-Os clock in crude oil, provided that $\mathrm{Re}$ is readily transferred from formation waters to oil (Hurtig et al., 2019). However, for oils with low asphaltene content $(\leq 0.0002 \mathrm{wt} \%)$ and petroleum systems with low water to oil ratios $(<150)$, there is a kinetic barrier for Re transfer at the oil-water interface, limiting the likelihood of resetting the Re-Os clock through this mechanism (Hurtig et al., 2019). The findings of these experimental studies have, however, not been observed in natural systems (Liu et al., 2018; Hnatyshin et al., 2019).

\subsubsection{Os isotope fingerprinting of oils to inferred source rocks}

Hydrous pyrolysis experiments simulating processes in natural petroleum systems have suggested that the Re-Os isotope system is not affected by thermal maturation of organic matter, and that the ${ }^{187} \mathrm{Os} /{ }^{188} \mathrm{Os}$ of crude oil at the time of its generation faithfully reflects that of the source rock (Creaser et al., 2002; Rooney et al., 2012; Cumming et al., 2014; Liu et al., 2018). The Re-Os isotope system would thus provide a powerful tool for tracing oil to its source, showing particular promise for biodegraded oils that may have partly or fully lost the commonly used organic tracers (Biomarkers; Killops et al., 1997; Peters et al., 2005; Sykes and Zink, 2008; Sykes et al., 2012). For example, the heavily biodegraded oil sands of the Western Canada Sedimentary Basin were shown to have been predominantly sourced from the Gordondale Formation and not the previously postulated Exshaw Formation using the Os isotope fingerprinting tool (Finlay et al., 2012). This fingerprinting tool has also been successfully applied to lacustrine Green River petroleum system for different phases of petroleum (crude oil, tar sands and gilsonite; Cumming et al., 2014), oils from the UK Atlantic 
margin (Finlay et al., 2011), oils from the Duvernay petroleum system in Western Canada Sedimentary Basin (Liu et al., 2018), bitumen and oil seeps from the Longmen Shan thrust belt, southwest China (Ge et al., 2018) and asphaltites from southern Australia (Corrick et al., 2019; Scarlett et al., 2019).

\subsection{New Zealand source rocks and oils}

A brief overview of key New Zealand organic-rich sedimentary rocks and their related hydrocarbon shows is presented here for background context, with more specific details given in subsequent chapters. Most commercial oil and gas accumulations discovered in New Zealand have been geochemically-typed to late Cretaceous-Eocene coals and coaly mudstones (Killops et al., 1994; King and Thrasher, 1996; Sykes et al., 2012). All of these accumulations are currently restricted to the Taranaki Basin, which is located both onshore and offshore along the western side of New Zealand (Fig. 1.6). This basin formed through continental rifting in the late Cretaceous as Zealandia broke away from Australia and Antarctica, creating deep faultcontrolled sub-basins that were filled with terrestrial and coastal plain sediments (Thrasher, 1992; King, 2000). The coaly source rocks of the Rakopi and North Cape formations were deposited at this time (Fig. 1.7; King and Thrasher, 1996). These rocks are widespread in the basin and comprise coals and coaly mudstones deposited in fluvio-deltaic settings with varying degrees of marine influence. Initially, the Rakopi Formation was considered almost entirely terrestrial (Titheridge, 1977; King and Thrasher, 1996). However, greater marine influence has been recognised within this formation as evidenced by elevated coal sulfur content and the presence of dinoflagellate cysts and glauconite (Sykes et al., 2004; Browne et al., 2008). The predominantly marine North Cape Formation includes the Wainui and Puponga coal measures that were deposited in a lower coastal plain setting (King and Thrasher, 1996; Sykes and Raine,

2008). The distribution of these coal measures is poorly defined because of a complex history of marine transgressive and regressive events within the North Cape Formation (Sykes and Raine, 2008). 


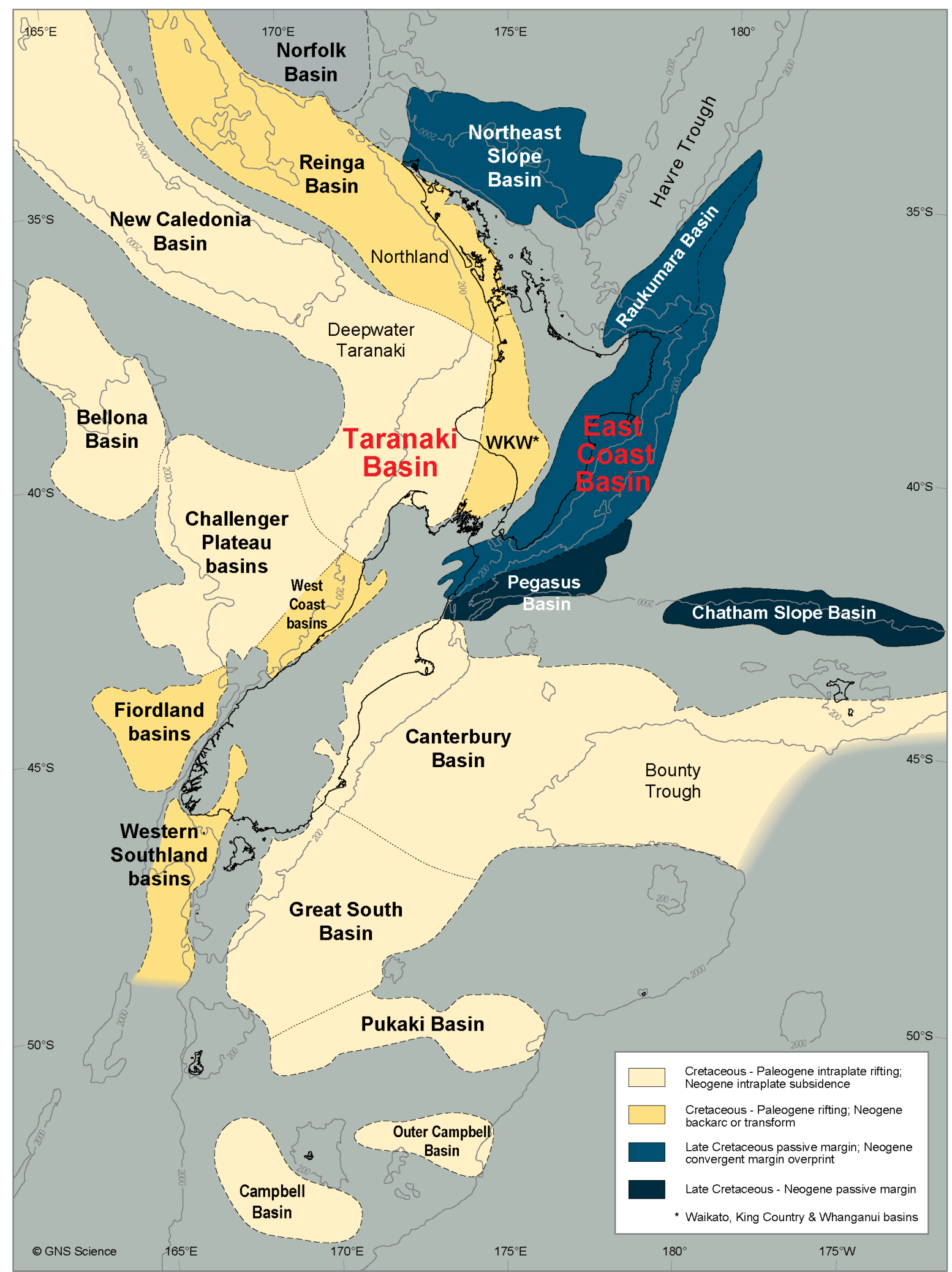

Fig. 1.6. A map showing sedimentary basins of New Zealand. The samples studied in this thesis were obtained from the Taranaki and East Coast basins, highlighted in red font. Adapted from New Zealand Petroleum and Minerals (2014). 


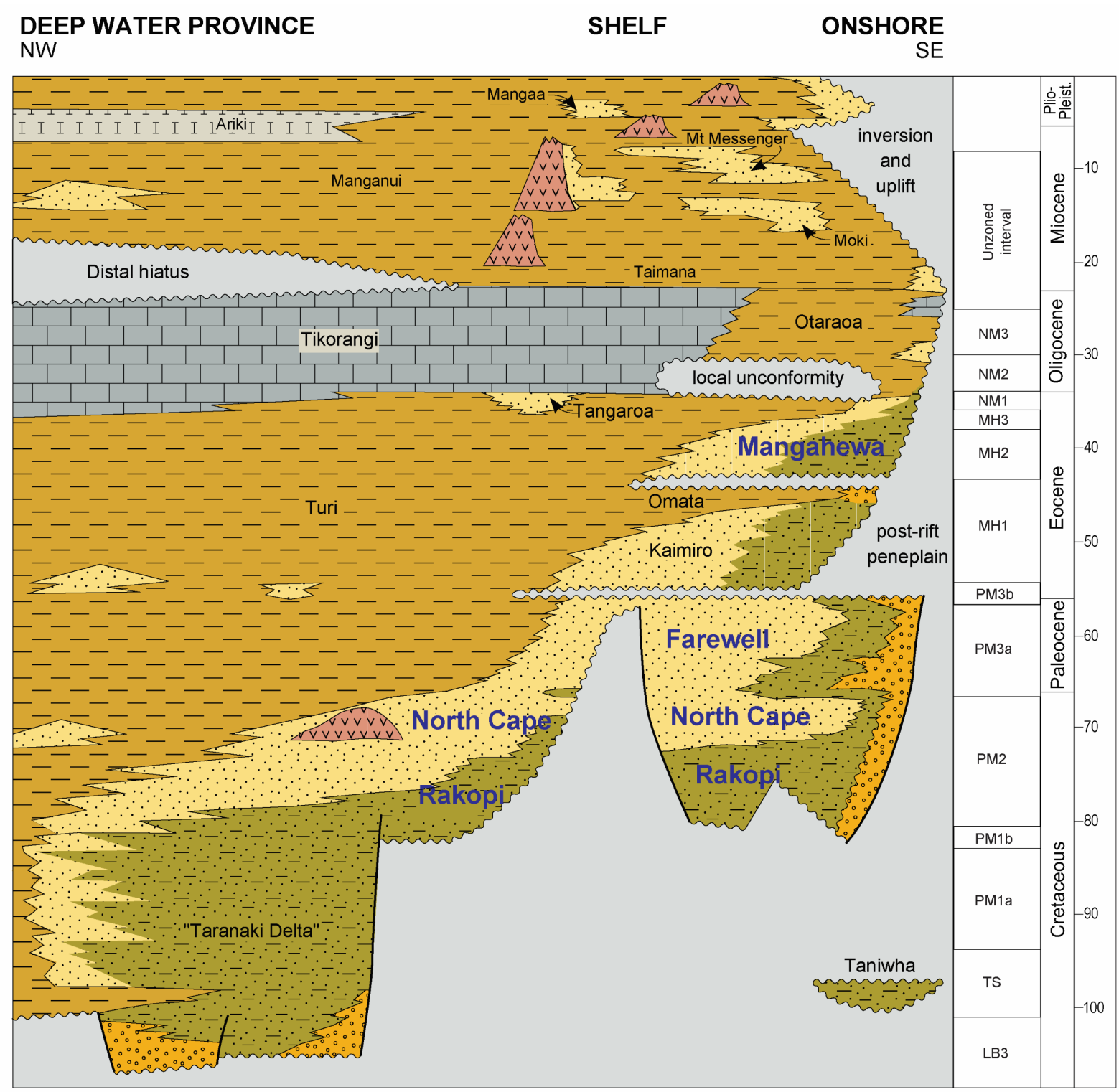

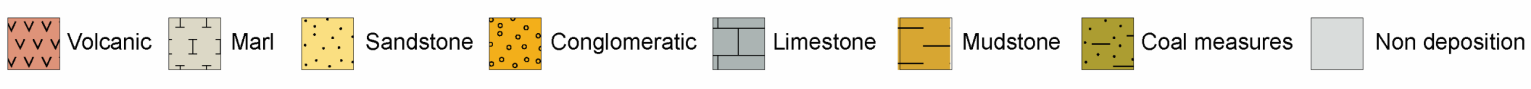

Fig. 1.7. A generalized chronostratigraphic panel of the Taranaki Basin across a northwest-southeast transect. Formations of interest to this thesis are highlighted in blue font. Adapted from New Zealand Petroleum and Minerals (2014).

The Rakopi and North Cape coals and coaly mudstones typically have TOC and hydrogen index (HI) values that range from 2 to $80 \%$ and 200 to $450 \mathrm{mg} \mathrm{HC} / \mathrm{g} \mathrm{TOC}$, respectively, indicating mixed gas- and oil-prone to oil-prone kerogen (King and Thrasher, 1996; Sykes et al., 2004; Browne et al., 2008; Sykes and Raine, 2008; New Zealand Petroleum and Minerals, 2014). They are modelled to be thermally mature for oil and gas generation in a number of kitchens in the southern part of the Taranaki Basin, and are inferred to have sourced oils in the 
Maui, Tui, Maari and Manaia fields (Appendix 1; Killops et al., 1994; Funnell et al., 2001; 2004; Sykes et al., 2012; Kroeger et al., 2016; Reilly et al., 2016). This inference is based on the relative abundance of source-specific terpane biomarkers that are diagnostic of higher plant evolution. A change in dominance of gymnosperm-derived diterpanes (e.g., isopimarane) in the late Cretaceous to angiosperm-derived triterpanes (e.g., oleanane) in the Eocene is noted in the source rocks and oils (Killops et al., 1995).

New Zealand oils are grouped into five genetic oil tribes and 11 families based on similarities of source-specific and maturity biomarkers. Four of these tribes are sourced from terrestrial coal-bearing sequences while the remaining one is sourced from marine rocks. The oils in the Maui, Tui, Maari and Manaia fields are genetically grouped as Family 11 oils and are inferred to have been sourced from the Rakopi and North Cape coals and coaly mudstones. These oils consistently have higher diterpane biomarkers compared to other coaly-sourced oils in the Taranaki Basin, indicating they are sourced from Cretaceous sediments.

Rifting around Zealandia had ceased by the end of the Cretaceous, resulting in continued marine transgression and deposition of Paleocene-Eocene sedimentary successions on broad coastal plains and an extensive shallow marine shelf (King, 2000; Higgs et al., 2012). The distribution of coaly source rocks in the Paleogene strata of the Taranaki Basin reflects the general extent of these marine transgressive events, with terrestrial sediments progressively dominating towards the south-eastern part of the basin (Killops et al., 1994; King and Thrasher, 1996). The Paleocene Farewell Formation generally contains fewer coaly (more clastic) source rocks, with varying degrees of marine influence. The Eocene Mangahewa Formation, on the other hand, is particularly coal-rich and consists of up to $\sim 1000 \mathrm{~m}$ of sediments in two major facies associations (Killops et al., 1994; King and Thrasher, 1996; Higgs et al., 2012; Sykes et al., 2014). The first facies association consists of coal measures deposited on broad, low-lying coastal plain environments. This interfingers to the northwest with the second comprising marginal marine sediments deposited in shoreface, lagoon, fluvio-deltaic and tidal environments (Flores, 2004; Higgs et al., 2012; Sykes et al., 2014). Coals and coaly mudstones are most prominent in the upper part of this formation, where they exhibit intermittent marine influence as evidenced by sparse occurrences of dinoflagellate cysts, common Ophiomorpha (trace fossil burrows), and elevated sulfur contents (0.9-62.6\% dry ash free [daf]; Sykes et al., 1999; 2014; Higgs et al., 2012). The Paleocene to Eocene coaly source rocks are generally 
more gas-prone than those of the late Cretaceous, however, they are inferred to have sourced oils in the McKee, Kupe, Kapuni and other onshore fields (Appendix 1; Killops et al., 1994; Sykes et al., 2012).

Marine organic-rich source rocks that are known to have generated oils in New Zealand are currently restricted to late Cretaceous-Paleocene sediments, the Whangai and Waipawa formations (Fig. 1.8; Hollis and Manzano-Kareah, 2005; Hollis et al., 2006; 2014; Naeher et al., 2019). The Waipawa Formation is a late Paleocene, widespread, thin $(<80 \mathrm{~m})$, massive to poorly bedded marine mudstone that occurs in numerous sedimentary basins around New Zealand and further afield on the eastern margin of Tasmania (e.g., Schiøler et al., 2010; Hollis et al., 2014; Naeher et al., 2019). It is relatively rich in organic matter with average TOC values of $3.6 \mathrm{wt} \%$ (range of $1-5 \mathrm{wt} \%$ ), and has moderate to good potential for generating petroleum (Killops et al., 1994; Field et al., 1997; Hollis and Manzano-Kareah, 2005; Naeher et al., 2019). It is inferred to have sourced sub-commercial oil in the Kora field in the northern part of the Taranaki Basin as well as several oil seeps in the East Coast Basin (Killops et al., 1994; Field et al., 1997; Rogers et al., 1999; Sykes et al., 2012). Despite deposition in a marine setting, the organic matter in the Waipawa Formation was derived mainly from terrestrial plants (Killops et al., 2000; Schiøler et al., 2010; Hollis et al., 2014; Naeher et al., 2019), indicating that unusual climatic and oceanographic conditions likely prevailed during its deposition (Hollis et al., 2014).

The Whangai Formation underlies the Waipawa Formation (Fig. 1.8) and is a late Cretaceous to late Paleocene, thick (up to $1500 \mathrm{~m}$ ) siliceous marine mudstone with widespread glauconitic sandstone and calcareous mudstone facies (Hollis and Manzano-Kareah, 2005). It comprises four members representing different facies and potential for sourcing hydrocarbons: Rakauroa, Te Uri, Porangahau and Upper Calcareous. Overall, this formation has low to moderate source rock potential, with average TOC and HI values of 0.56 (range of $0.1-1.7$ ) wt $\%$ and 159 (range of 16-377) mg HC/g TOC, respectively (Moore, 1988; Field et al., 1997; Hollis and ManzanoKareah, 2005). It is inferred to have sourced oil seeps in the northern and southern parts of the East Coast Basin (Rogers et al., 1999; Sykes et al., 2012). 


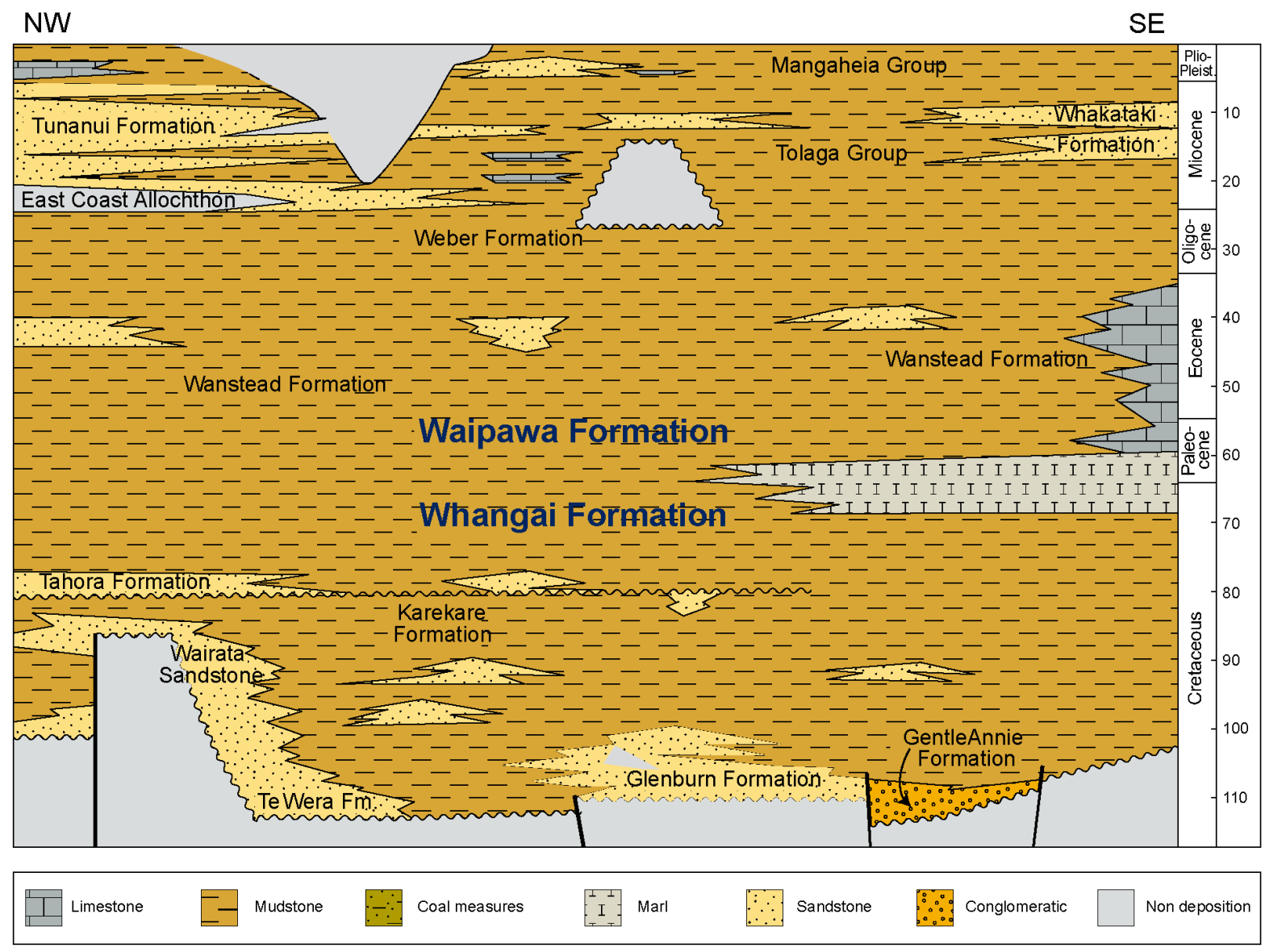

Fig. 1.8. A generalized chronostratigraphic panel of the East Coast Basin across a northwest-southeast transect. The formations of interest (Waipawa and Whangai) are highlighted by the blue font. Adapted from New Zealand Petroleum and Minerals (2014).

\subsection{Thesis overview and rationale}

The research undertaken in this thesis is presented in three main chapters formatted as scientific papers, one of which has been published at the time of thesis submission. These three studies seek to better understand the factors controlling Re-Os systematics in New Zealand's terrestrial and marine organic-rich sedimentary rocks and related oils, and evaluate whether they are amenable to dating using the Re-Os system. The thesis comprises this introductory chapter, a methodology chapter, three main research chapters in the format of journal manuscripts and a conclusion chapter. As the three main chapters have been written as stand-alone studies, some minor repetition exists, particularly in the methodology sections. The contents of the chapters are briefly outlined below. 


\subsubsection{Chapter Two: Methodology}

The methodology chapter of this thesis provides a background to the principles behind the development of the current procedures for Re-Os sampling, sample preparation and isotopic analysis. Rhenium and Os analysis involve complex and time-consuming processes, and it is imperative that careful sampling and sample preparation protocols are followed to ensure data quality and accuracy. The parameters that were considered for obtaining and processing samples for Re-Os analysis are outlined, as well as advances that were made in sample digestion, separation protocols and mass spectrometric analysis to address specific issues and peculiarities of the New Zealand sample suites. The theory and principles behind data reduction steps and regression methods used in the thesis are also outlined in this chapter.

\subsubsection{Chapter Three: Re-Os geochronology and isotope systematics, and organic and sulfur geochemistry of the middle-late Paleocene Waipawa Formation, New Zealand: Insights into early Paleogene seawater Os isotope composition}

This chapter investigates how the dominance of terrestrial organic matter, high sedimentation rates and hypoxic conditions in the Waipawa Formation may have affected its Re-Os uptake and isotope systematics. This formation has attracted increasing scientific interest due to its significance for regional and global reconstructions of the early Paleogene transitional climate as well as for its potential for oil and gas exploration. Re-Os, bulk pyrolysis and sulfur data were obtained for this formation and the underlying Whangai Formation that exhibits contrasting organofacies (dominance of marine organic matter) and depositional conditions (slow sedimentation rates and oxic environment). This study also uses the Re-Os geochronometer to provide the first direct radiometric depositional age for the Waipawa Formation.

Furthermore, determination of Os isotopic composition of seawater at the times of Waipawa and Whangai formation deposition, allowed for an assessment of factors controlling the evolution of seawater Os curve in the early Paleogene. This period records dramatic fluctuations in the Earth's climatic and oceanographic settings, which are reflected in the marine Os record. This chapter presents a composite record of the marine Os curve for the first $10 \mathrm{Ma}$ of the Paleogene, incorporating all data that have been produced since the publication 
of the first synoptic record more than two decades ago and adding the Osi values obtained from the Waipawa and Whangai formations.

\subsubsection{Chapter Four: Depositional influences on Re-Os systematics of late Cretaceous- Eocene fluvio-deltaic coals and coaly mudstones, Taranaki Basin, New Zealand}

This fourth chapter investigates factors controlling Re-Os systematics in sedimentary rocks deposited in freshwater and marine-influenced fluvio-deltaic environments, as typified by the Cretaceous-Eocene coal measures in New Zealand. To date, only three Re-Os studies have been published on fluvio-deltaic (coaly) sedimentary successions. These studies suggest that marine influence may be required for these types of rocks to be amenable to the Re-Os geochronometer, as it may allow significant enrichment of well-mixed (homogenised) Os

needed for precise Re-Os geochronology. It is, however, unclear whether this applies to all marine-influenced coaly sediments or under what conditions. This chapter presents Re-Os, bulk pyrolysis and sulfur data for late Cretaceous-Eocene coals and coaly mudstones from the Rakopi, North Cape, Farewell and Mangahewa formations in the Taranaki Basin, New Zealand. These coals and coaly mudstones were deposited in various fluvial, estuarine and coastal plain environments, and exhibit varying degrees of marine influence.

\subsubsection{Chapter Five: Re-Os systematics in marine and terrestrial oils from the Taranaki and East Coast basins, New Zealand}

This chapter assesses for the first time the behaviour of $\mathrm{Re}$ and Os in oils sourced from terrestrial coals, and the potential of the Re-Os isotope system to date and correlate these oils to their source(s) by targeting oils (oil Family 11) that have been linked to the source rocks evaluated in chapters 4 (Rakopi and North Cape formations). The ${ }^{187} \mathrm{Os} /{ }^{188} \mathrm{Os}$ isotope compositions of the oils and the two formations at the time of oil generation (10 Ma) are compared. In addition, Re-Os analyses were carried out on a family of very distinctive Taranaki Basin oils (Family 41) with biomarker parameters that indicate derivation from a source with both marine and terrestrial organic matter. These oils have been correlated to the Waipawa Formation based on their particularly heavy carbon isotopic signature in comparison to other New Zealand marine oils (Family 42) that seep out on the northern and southern parts of the East Coast Basin. The Family 42 oils were also analysed for their Re-Os concentrations and isotopic compositions. 


\subsubsection{Chapter Six: Conclusions and outlook}

This final chapter summarizes the work undertaken in the main research chapters of this thesis. In addition, this chapter provides insights and suggestions for future work that would build on the research findings presented herein. 


\section{Methodology}

Specific sample processing and analytical methodologies for the marine shales, coaly sediments, and oils, are described in each of the main research chapters (Chapters 3-5), respectively. This chapter provides a brief overview of the methods, together with background context and theoretical considerations. The Re-Os isotopic analyses of organic-rich sedimentary rocks and crude oils are undertaken in four steps: (1) sample preparation which includes rock powdering and asphaltene precipitation, (2) acid dissolution and equilibration of sample and spike, (3) chemical separation and purification of Re and Os from the matrix and each other, and (4) mass spectrometric measurement of Re and Os isotopes (Fig. 2.1).

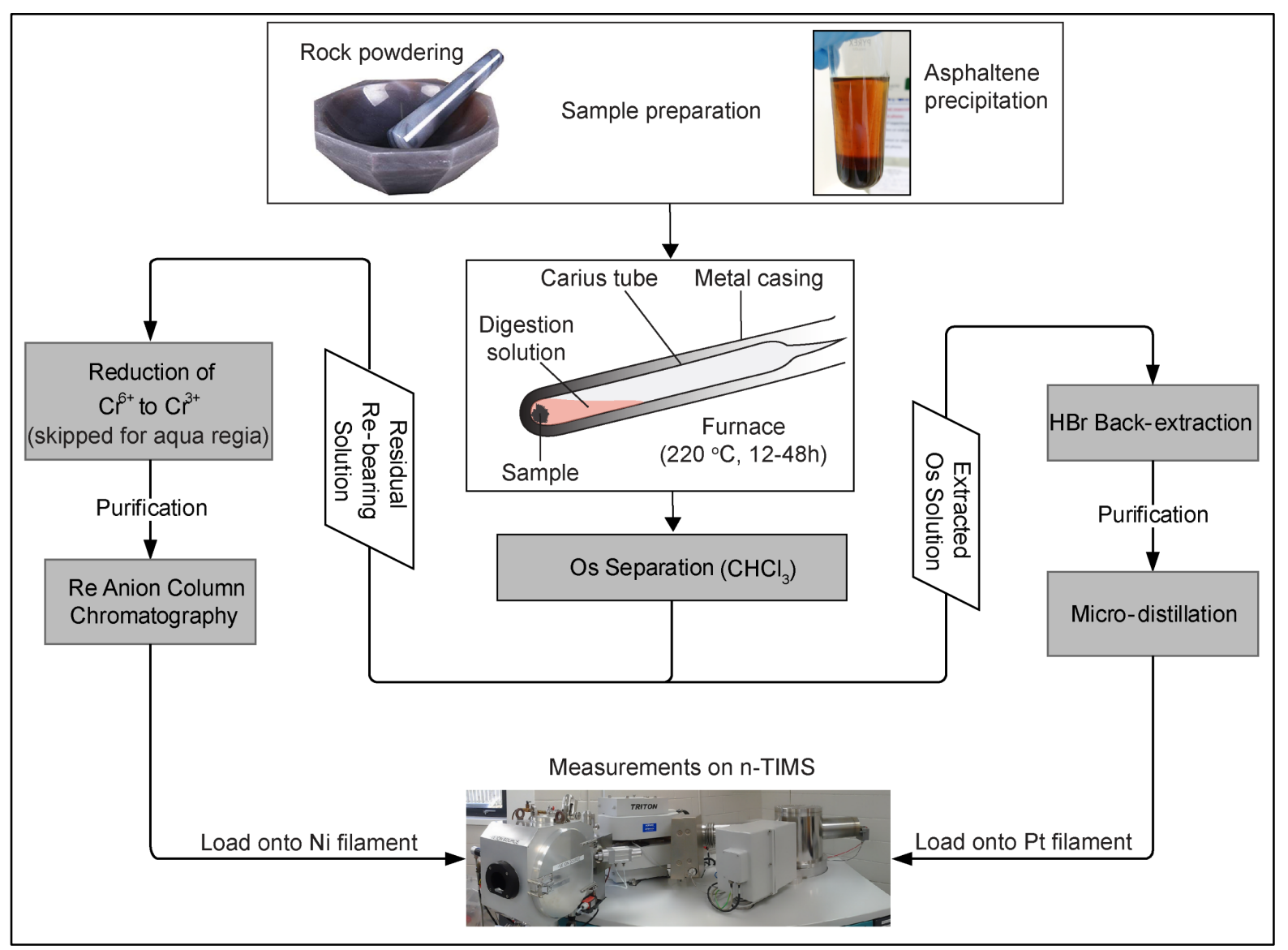

Fig. 2.1. Flowchart summarising the Re-Os methodology from sample preparation to isotopic measurement on a negative thermal ionisation mass spectrometer. 


\subsection{Rock sampling and processing strategy}

Production of precise and accurate Re-Os data is highly dependent on following appropriate sampling protocols. This is especially important for outcrop samples because post-depositional processes such as oxidative weathering have been shown to severely affect the integrity of the Re-Os isotope system (Peucker-Ehrenbrink and Hannigan, 2000; Jaffe et al., 2002; PiersonWickmann et al., 2002; Georgiev et al., 2012). For example, Pierson-Wickmann et al. (2002) showed that oxidative weathering can occur up to $5 \mathrm{~m}$ depth from the surface of an outcrop, even in samples that appear unweathered upon visual inspection. Fresh drill core samples are, therefore, preferred to outcrop samples. There are instances, however, where only outcrop samples are available, as is the case for the Rakopi and North Cape formations in this study. In such cases, care must be taken to remove all weathered material (generally recognized by, for example, lighter colours, friable appearance, jarosite stains or gypsum laths) prior to sampling and during sample preparation. By following such protocols, studies have successfully obtained precise and reliable Re-Os data using outcrop samples (e.g., Xu et al., 2009; Tripathy et al., 2014).

Two sampling strategies were employed while obtaining rock samples for this research project. One strategy addressed the aim of evaluating the behaviour of $\mathrm{Re}$ and Os in different depositional settings. The other addressed the aim of testing the Re-Os isotopic system as a geochronometer for both fluvio-deltaic coal measures and marine shales of Waipawa Formation.

\subsubsection{Sampling to evaluate Re-Os systematics in different depositional settings}

New Zealand sedimentary basins contain rock formations that were deposited in a range of environments including fluvial, estuarine, marginal marine, shallow (shelf-slope) marine and deep-bathyal settings (e.g., King and Thrasher, 1996; Field et al., 1997; Higgs et al., 2012). Core and outcrop samples for these formations in the Taranaki and East Coast basins were available for selection in this project (Fig. 2.2). Rock samples were selected to target intervals with varying depositional environments and levels of marine influence, allowing the impact of these factors on the behaviour of Re and Os to be investigated. The selected coals and coaly mudstones exhibit the full range of marine influence, from purely terrestrial to slightly, moderately and strongly marine-influenced. Some of these coals and coaly mudstones were 
deposited in low-lying swamps that were proximal to active channels and others in distal floodplain lake settings, allowing for the effects of high and low energy clastic sedimentation on Re-Os uptake and fractionation to be evaluated. Targeted sampling also aimed at investigating whether episodic influxes of large amounts of terrestrial material during deposition of the marine Waipawa Formation (Naeher et al., 2019) may have supplied high loads of continental-derived Os and locally influenced seawater Os compositions.
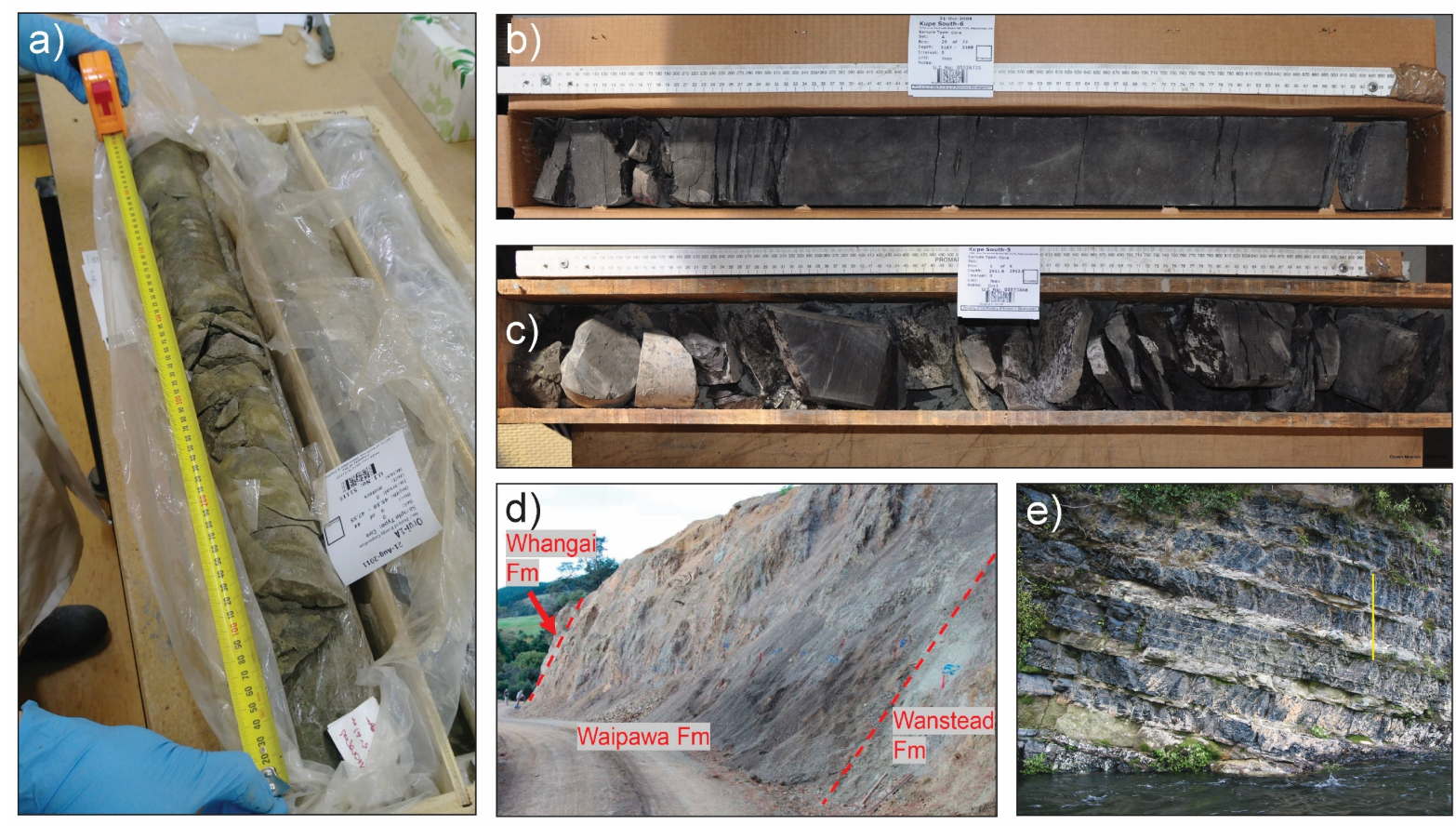

Fig. 2.2. Photographs of some core materials and outcrops that were available for this project a) The Waipawa Formation in Orui-1A core between $45.6 \mathrm{~m}$ and $47.6 \mathrm{~m} . \mathrm{b}$ ) and c) The Farewell Formation in Kupe South-6 (3167-3168 m) and Kupe South-5 (2911.8-2912.9 m), respectively. d) Outcrop photograph of the Taylor White section with approximate boundaries between Whangai, Waipawa, and Wanstead formations (Bland et al., 2014; Naeher et al., 2019). e) Outcrop photograph of the Rakopi Formation in the upper reaches of the Paturau River in northwest Nelson. The yellow scale bar is approximately $1 \mathrm{~m}$ long (Sykes et al., 2004).

\subsubsection{Sampling for Re-Os geochronology}

For geochronology, sampling should be limited to the smallest possible stratigraphic (time) interval to avoid the risks of compromising isochroneity due to potential variations in the $\mathrm{Osi}_{\mathrm{i}}$ composition. Ideally, samples should be obtained from an interval of $<1 \mathrm{~m}$. However, sampling from a very small stratigraphic interval may also increase the risk of capturing small-scale postdepositional isotopic exchange that occurs due to redistribution of organic particles by burrowing organisms at the bottom of the water column (Fig. 2.3; Stein and Hannah, 2014). 


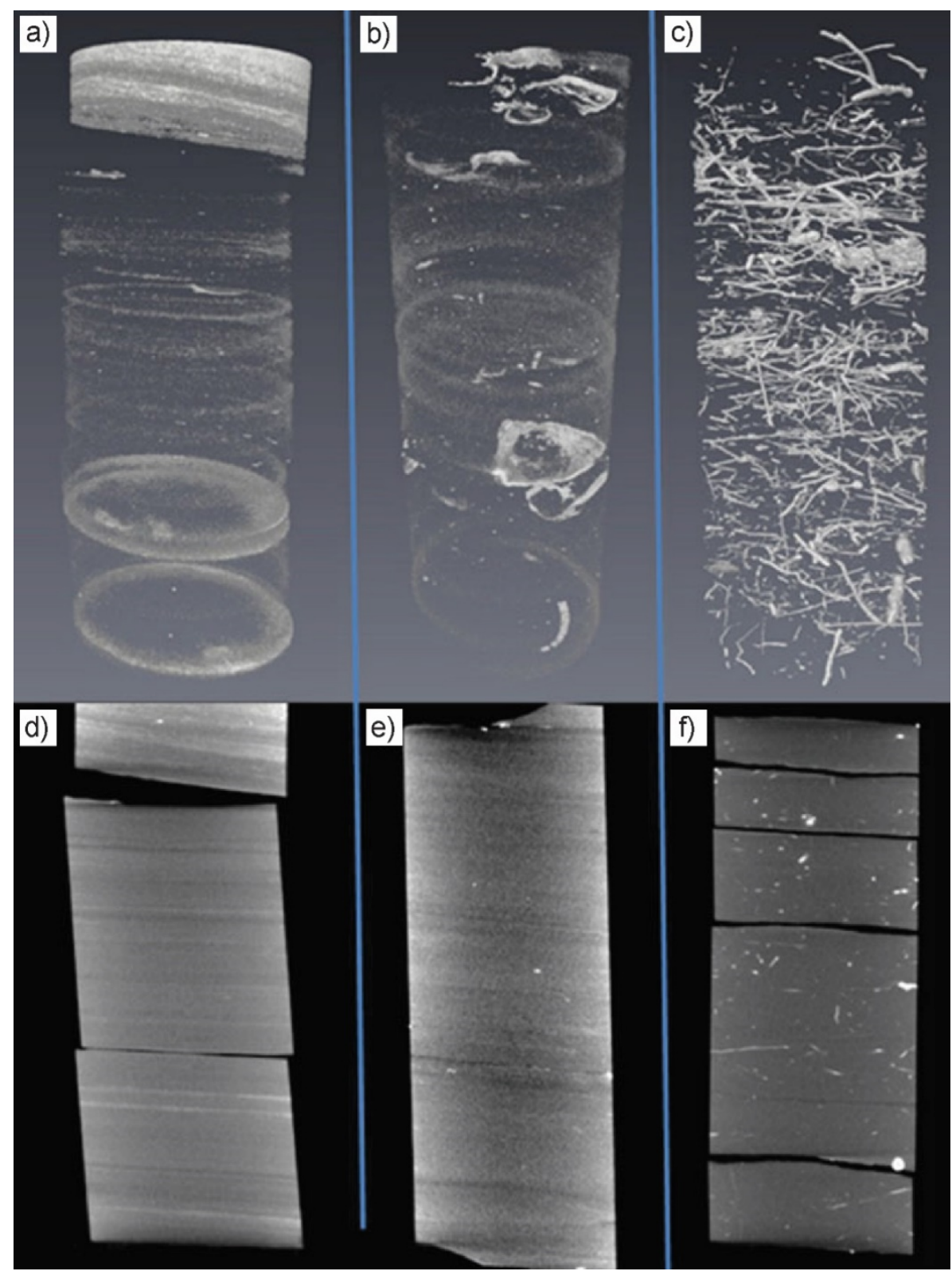

Fig. 2.3. Computerized tomography images $(a, b, c)$ and corresponding photographs $(d, e, f)$ for three intervals from a drill core, showing the importance of carefully selecting samples for Re-Os geochronology (Stein and Hannah, 2014). a, d) Ideal core material with well-laminated organic-rich shale interbedding with siltier, less organic-rich intervals. b, e) Suitable core material with brachiopod fossils and pyritized foraminifera (scattered bright points) indicating stable and anoxic-euxinic depositional conditions, respectively. c, f) Less desirable material as it is intensely bioturbated with a myriad of fossil burrows.

This mainly affects sediments deposited in well-oxygenated bottom waters where burrows thrive (Collinson, 2005). In this thesis, sampling for geochronology was limited to a stratigraphic interval of $\sim 3 \mathrm{~m}$. This larger interval than the recommended $<1 \mathrm{~m}$ was necessary due to small variations in ${ }^{187} \mathrm{Re} /{ }^{188} \mathrm{Os}$ ratios in the sampled intervals. 
Typically, a sample suite of 5 to 8 is required for development of an isochron. However, each sampling interval is different and requires its own strategy to obtain the most precise and accurate ages. Sedimentation rates, variation of Osi ratios, spread in ${ }^{187} \mathrm{Re} /{ }^{188} \mathrm{Os}$ ratios and the abundance of Re and Os are other factors to be taken into consideration. In this thesis, 5 to 9 samples were selected per interval.

\subsubsection{Sample processing}

Once a suitable interval has been sampled, rock samples should be powdered without metal contact. This is because Re and Os are siderophile elements and can therefore be contaminated by conventional rock-crushing steel mills or crushers (Creaser et al., 2002). In this project, samples were first cleaned with ultra-pure water to remove any potential surface contamination for outcrop samples and drilling mud for core samples. Core samples were then polished on a diamond plate lapping machine to remove drilling marks. Clean samples were dried overnight in an oven at $40{ }^{\circ} \mathrm{C}$ and then roughly crushed using a hydraulic press into smaller pieces for easier powdering on an agate mill. Although there is no threshold amount of rock that should be pulverized and homogenized, Kendall et al. (2009b) suggested that sample powders representing $\geq 20 \mathrm{~g}$ of rock are preferable to remove the effect of small-scale diffusion and element decoupling. The final weight of the powdered samples in this project ranged between 20 and $40 \mathrm{~g}$. More detailed processing protocols for the different rock types are provided in Chapters 3 and 4.

For crude oils, the samples were stored in well-labelled glass bottles, with Teflon-lined lids. In addition, the sample bottles were stored in a cool and dark place to reduce evaporative changes or microbial degradation of the oil.

\subsection{Asphaltene precipitation from crude oil}

Crude oil can be classified into two main fractions - maltenes and asphaltenes - based on solubility in excess apolar solvents (e.g., n-pentane, $n$-heptane). Maltenes are the $n$-alkane soluble fraction and are composed of saturates, aromatics and resins. Asphaltenes are the $n$ alkane insoluble high-molecular-weight components of crude oil that are dark brown to black in colour and have no definite melting point, usually forming a carbonaceous residue upon heating (Speight, 2004). The asphaltene fraction is utilised for Re-Os studies because it 
contains the majority (52-98\%) of Re and Os, and in most cases, its ${ }^{187} \mathrm{Re} /{ }^{188} \mathrm{Os}$ and ${ }^{187} \mathrm{Os} /{ }^{188} \mathrm{Os}$ are comparable to those of the whole oil (Selby et al., 2007a; Georgiev et al., 2016). Therefore, separation of the asphaltene fraction from crude oil effectively pre-concentrates Re and Os, allowing for more precise Re-Os analysis.

The asphaltene fraction is typically separated from crude oil using either $n$-pentane or $n$ heptane (Speight, 2004; Selby et al., 2007a). The choice of the solvent can significantly influence the asphaltene yield because, for a given crude oil-solvent dilution ratio, the quantity of asphaltene precipitated decreases with increasing chain length of the $n$-alkane. From this consideration, $n$-pentane would therefore be the preferred precipitant to use rather than $n$ heptane as it leads to greater precipitation of asphaltenes. However, $n$-pentane is highly volatile and thus, more difficult and less safe to handle during experiments, especially when processing high wax oils that require some form of heating during the separation (see below).

For this thesis, the asphaltene separation process involved thoroughly mixing (in a $60 \mathrm{ml}$ glass vial) $1 \mathrm{~g}$ of crude oil in $40 \mathrm{ml}$ of $n$-heptane and gently shaking the mixture on an orbital shaker overnight (at least $12 \mathrm{~h}$ ) at room temperature (Selby et al., 2007a). The contents of the vial were then centrifuged at $3500 \mathrm{rpm}$ for 10-15 minutes to separate the precipitated asphaltene from the soluble maltene fractions (Fig. 2.4). The supernatant (maltene fraction) was decanted to waste while the remaining residue (asphaltene fraction) was transferred into a pre-weighed glass vial using $\leq 1 \mathrm{ml}$ chloroform $\left(\mathrm{CHCl}_{3}\right)$. Use of a small amount of $\mathrm{CHCl}_{3}$ is necessary to keep $\mathrm{Re}$ and Os blank contributions to a minimum. The $\mathrm{CHCl}_{3}$ was then evaporated overnight on a hot plate at $60^{\circ} \mathrm{C}$. All the above sample preparation steps were performed in a fume hood, with all personal protective equipment worn (i.e., gloves, eye goggles and lab coat).

New Zealand crude oils are typically sourced from terrestrial coal measures, which led to two significant analytical challenges in this project. First, many of these oils are solid at room temperature due to their high wax content and abundant terrestrial organic matter (Fig. 2.4; Sykes and Zink, 2012; Sykes et al., 2012). The first step, therefore, required the highly viscous, solid stock oils to be heated to about $50{ }^{\circ} \mathrm{C}$ to obtain a viscous liquid. This was then thoroughly mixed to remove any gravitational segregation of oil fractions during storage. The required amount of the oil was then transferred into a pre-weighed $60 \mathrm{ml}$ vial using a pre-heated transfer pipette. Pre-heating of the transfer pipettes was necessary because the oils immediately solidified on touching cold surfaces. The need to heat the oil samples and vials to about $50{ }^{\circ} \mathrm{C}$ 
before adding the precipitant meant that only the less volatile $n$-heptane with a boiling point of $98.4{ }^{\circ} \mathrm{C}$, compared to the highly volatile $n$-pentane with a boiling point of $36.1{ }^{\circ} \mathrm{C}$ (e.g., Ouellette and Rawn, 2015), could be used for the precipitation process. Further, most of the high-wax oils did not dissolve entirely on addition of the $n$-heptane, requiring an additional step of first dissolving the crude oil in three to four drops of a more polar solvent (dichloromethane) before adding heated $n$-heptane (at $50^{\circ} \mathrm{C}$ ).

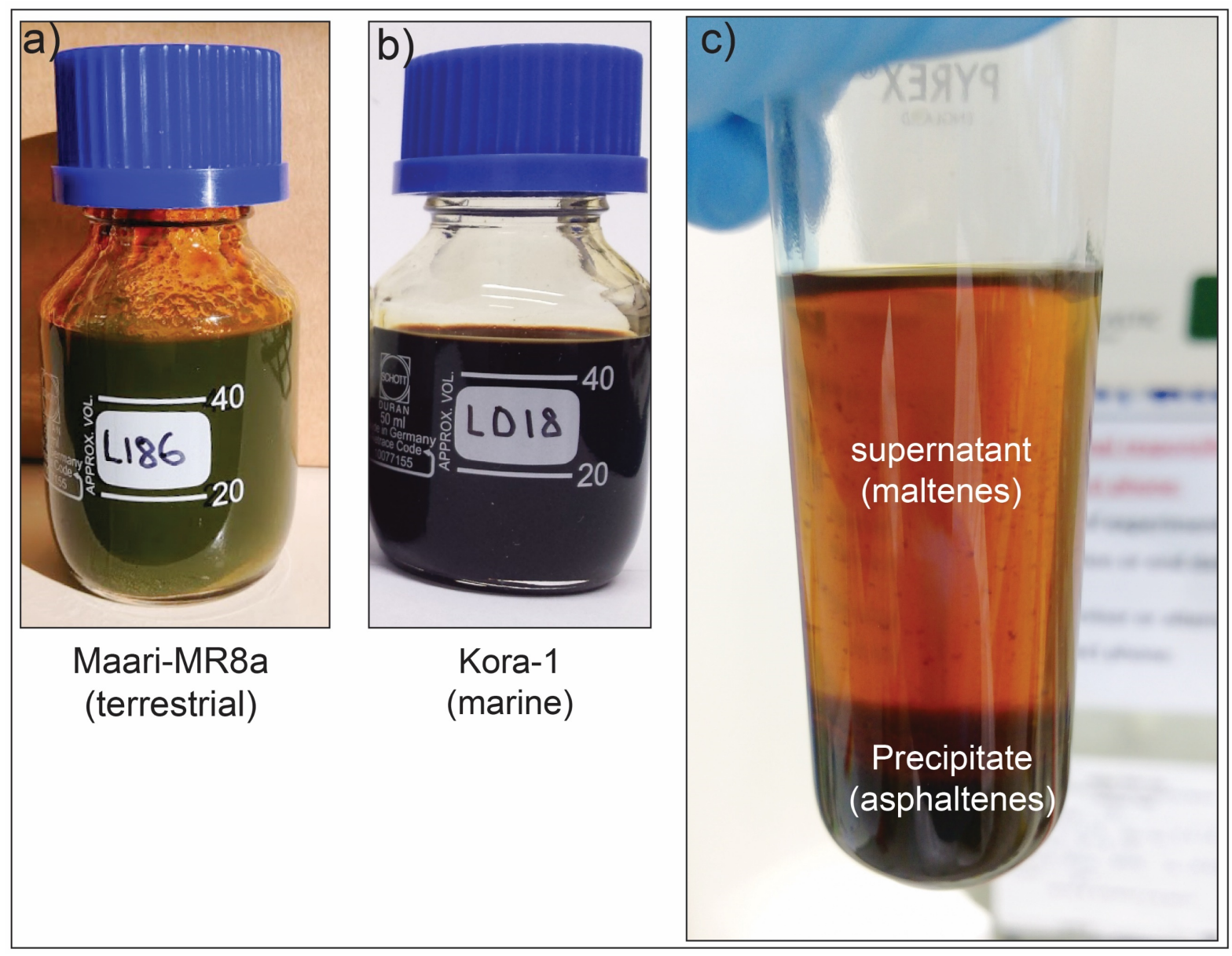

Fig. 2.4. a) and b) Photographs showing examples of terrestrial and marine oils, respectively, from the Taranaki Basin. The terrestrial oil (a) is highly viscous whereas the marine oil (b) is liquid at room temperature. c) A photograph of the asphaltene separation step using $n$-heptane.

The second main challenge with the terrestrial coaly-sourced oils was their low asphaltene contents, which typically range from 0 to $5 \%$ (Sykes and Zink, 2012). This meant that large quantities of the oil samples were required to obtain the required amount ( 250 $\mathrm{mg})$ of asphaltenes for Re-Os analysis (e.g., Liu et al., 2018), consuming a large amount of time and materials. In some cases, up to $30 \mathrm{ml}$ of crude oil was required to yield the $\sim 250 \mathrm{mg}$ of asphaltenes, requiring up to 1.2 litres of the solvent. The abundance of Re and Os in the maltene 
fraction was not determined in this project because the already preconcentrated asphaltene fractions contained very low abundance of the elements.

\subsection{Sample digestion and Re-Os separation techniques}

\subsubsection{Sample digestion}

The digestion techniques available for Re-Os analysis are constrained by the existence of Os in multiple oxidation states in solution (ranging from -2 to +8 ), which prevent complete mixing and equilibration of the spike and sample ratios during isotope dilution analysis (Dickin, 2018). In addition, loss of the highly oxidised and volatile Os species (OsO4; evaporation temperature of $130{ }^{\circ} \mathrm{C}$ at $10^{5} \mathrm{~Pa}$ ) is particularly problematic for open or Teflon-based digestion vessels. There are two broad approaches to overcoming the multiple oxidation states, dissolution methods that are either fully reducing (e.g., NiS fire assay) or highly oxidising in a fully sealed vessel (e.g., Carius tube, high pressure asher; Shirey and Walker, 1995; Shen et al., 1996; Ravizza and Pyle, 1997; Meisel et al., 2001). The Carius tube and high pressure asher methods are preferred for geological samples that typically have low Re and Os concentrations $(<10 \mathrm{ppt}$ to a few hundreds of $\mathrm{ppb}$ ) because they introduce minimal blank of the elements compared to NiS fire assay (Reisberg and Meisel, 2002). Minimal contamination is easily attained in the Carius tube and high pressure asher methods because high purity acids that are used in these methods are generally easier to obtain than high purity fluxes used in the NiS fire assay technique.

The Carius tube method, which was utilised in this study, enables digestion at high temperature and pressure in sealed glass ampoules (Carius tubes; Fig. 2.5a) without the loss of the volatile $\mathrm{OsO}_{4}\left(200-260{ }^{\circ} \mathrm{C}\right.$; Shirey and Walker, 1995). Acid dissolution of samples in sealed Carius tubes under high pressure and temperature is somewhat hazardous due to the risk of explosion. However, this rarely occurs if the tubes are not overfilled. Nonetheless, Carius tubes must always be heated in specifically designed closed steel jackets to minimize the release of the contents in case the tube fails (Fig. 2.5b). The steel jacket is made in such a way that its internal volume is twice the volume of the Carius tube to prevent it from being over-pressured in case the tube explodes. Further, heated Carius tubes must be handled with appropriate personal protective equipment and stored with care because they may be over-pressurized even after cooling to room temperature. Despite these challenges, the Carius tube method remains highly 
advantageous and the most commonly used method for Re-Os work due to its ability to achieve complete equilibration of sample-spike ratios (e.g., Selby and Creaser, 2003; Jones et al., 2018 and references therein).

a)

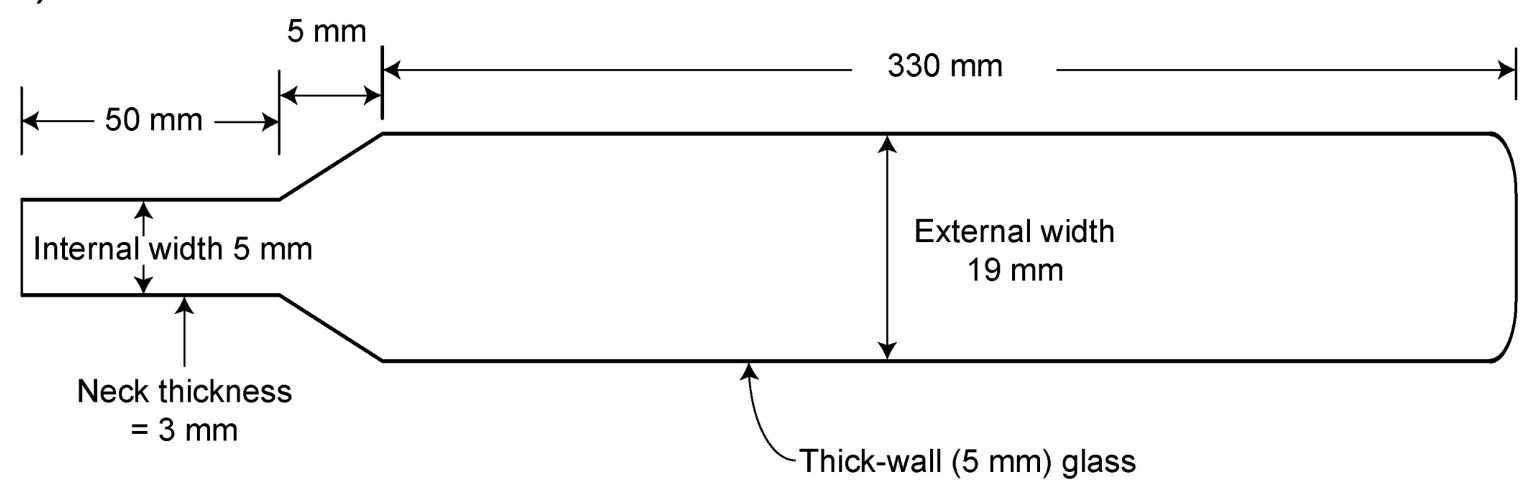

b)

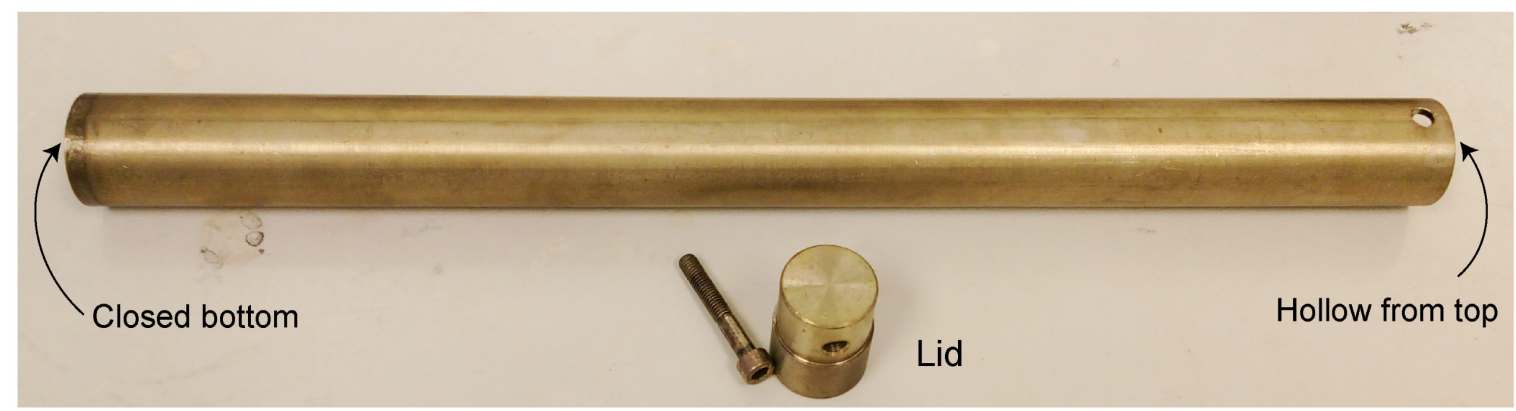

Fig. 2.5. a) Schematic diagram of a Carius tube employed in this thesis, with dimensions of the various components. b) A photograph of a metallic safety jacket used at Durham University for containing sealed Carius tubes during oven heating.

In this project, the asphaltenes were introduced into the thick-walled glass tube via a funnel or modified transfer pipette that is long enough to extend beyond the neck of the tube (Fig. 2.6a). This was necessary to prevent samples or reagents touching the neck, which would prevent tight sealing of the tube. As asphaltenes were transferred in liquid form using $\mathrm{CHCl}_{3}$, the subsequent drying step was undertaken while the top half of the Carius tube was elevated to prevent the liquid contents from flowing back to the neck of the tube (Fig. 2.6b). After the asphaltenes had completely dried, the spike and digestion solutions were added while the lower half of the Carius tube was immersed in a dry ice-ethanol slurry. This allowed the added mixture of acid, sample and spike to freeze, reducing premature reaction of the mixture and also reduces the vapour pressure of the reagents, making the tube sealing process easier and safer (Shirey and Walker, 1995). Liquid nitrogen should not be used to chill the tubes because 
once sealed, the tubes can become over-pressurized at room temperature and may explode when heated (Shirey and Walker, 1995). The combined mixture of acid and sample in the tube filled about one-third to one-half of the tube's volume.

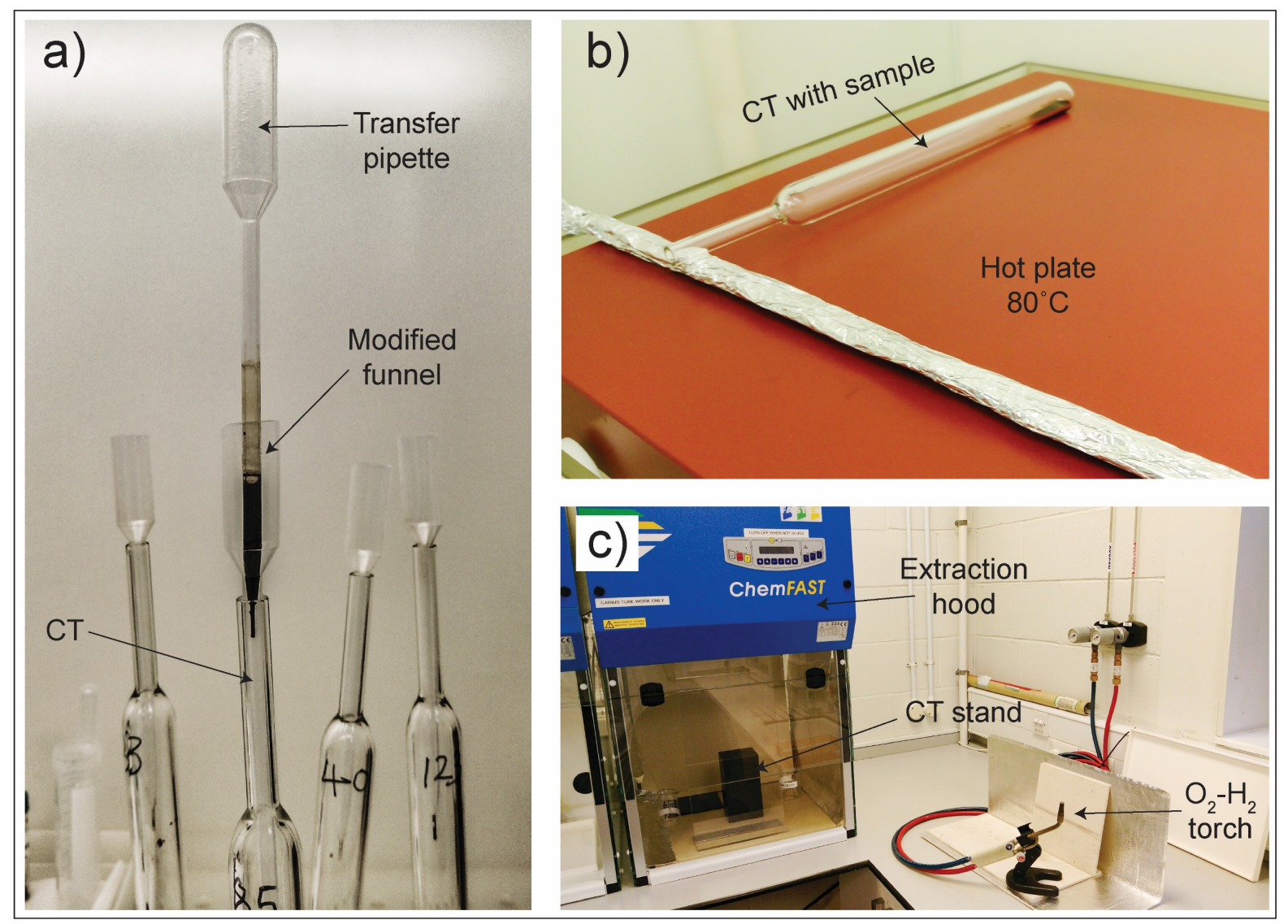

Fig. 2.6. a) A photograph showing the transfer of asphaltene into a Carius tube (CT) using a modified funnel (made by cutting the top a transfer pipette) to prevent samples touching the neck of the CT. b) Drying of the transferred asphaltene on a hot plate. A rolled aluminium foil is used to elevate the top half of the CT, ensuring that the liquid content remained at the bottom of the tube. c) CT sealing and opening station at Durham University's geochemistry laboratory.

At Durham University, where all Re-Os analyses for this thesis were undertaken, the Carius tubes are sealed using an oxygen-hydrogen torch (Fig. 2.6c). After sealing, the tubes were allowed to warm to room temperature, then placed in metallic safety jackets and heated in an oven to $220^{\circ} \mathrm{C}$ for $24-48 \mathrm{hrs}$, depending on sample material and acid combination used (see below). After the digestion was complete, the Carius tubes were opened by etching just below the neck, the contents re-frozen in a dry ice-ethanol slurry, and the tube vented by gently heating the neck with a torch until a small hole forms that releases internal pressure that may have formed. The upper part of the Carius tube was then broken off by placing a hot glass rod on the etched area, which causes a crack to propagate around the full circumference of the tube. 
Depending on sample material, Carius tube digestion involves the use of either inverse aqua regia (3 ml $12 \mathrm{~mol} \mathrm{l}^{-1} \mathrm{HCl}+6 \mathrm{ml} 16 \mathrm{~mol} \mathrm{l}^{-1} \mathrm{HNO}_{3}$ ) or $\mathrm{CrO}_{3}-\mathrm{H}_{2} \mathrm{SO}_{4}$ acid solution (Selby and Creaser, 2003). Re and Os in organic-rich sedimentary rocks are generally of hydrogenous origin being sequestered from a waterbody at the time of deposition (Cohen et al., 1999; Selby and Creaser, 2003). However, a non-hydrogenous (i.e., detrital) component of Os may be incorporated into organic-rich sedimentary rocks at the time of deposition (Creaser et al., 2002; Selby and Creaser, 2003). This detrital component introduces Os with a different timeintegrated history to the hydrogenous Os, and if it is included in the analysis will generally lower the overall accuracy of the data obtained and produce scatter in an isochron, which reduces the precision of the isochron age (Rooney et al., 2011). Selby and Creaser (2003) recommend the use of $\mathrm{CrO}_{3}-\mathrm{H}_{2} \mathrm{SO}_{4}$ solution for sample digestion of organic-rich sediments because this approach preferentially liberates the hydrogenous Re and Os, yielding more precise isochron ages, by effectively leaching the sample rather than a full dissolution.

For the most part, $200-500 \mathrm{mg}$ of rock powder was digested in this study using the $\mathrm{CrO}_{3}-$ $\mathrm{H}_{2} \mathrm{SO}_{4}$ solution. However, some coal samples were found to produce better results using inverse aqua regia, possibly due to the high TOC content in these samples. Details are given in Chapter 4. For oil samples, which are mainly products of the organic matter in sedimentary rocks, detrital material is very low to negligible and thus, inverse aqua regia is the preferred digestion solution (Selby et al., 2007a). Prior to use, both solutions were purified to lower blank contributions. $\mathrm{HCl}$ and $\mathrm{HNO}_{3}$ used in the aqua regia solution were purified by distillation in a closed Teflon system. Rhenium in the $\mathrm{CrO}_{3}-\mathrm{H}_{2} \mathrm{SO}_{4}$ solution was removed by solvent extraction using tetrapropyl ammonium iodide (TPAI), which binds $\mathrm{Re}$ in $4 \mathrm{~N} \mathrm{H}_{2} \mathrm{SO}_{4}$, with the TPAI extracted using $\mathrm{CHCl}_{3}$ (Shen et al., 1996; Selby and Creaser, 2003). For this procedure, $10 \mathrm{ml}$ of $2 \%$ TPAI in $4 \mathrm{~N} \mathrm{H}_{2} \mathrm{SO}_{4}$ and $50 \mathrm{ml} \mathrm{CHCl}_{3}$ was added to $200 \mathrm{ml}$ of $\mathrm{CrO}_{3}-\mathrm{H}_{2} \mathrm{SO}_{4}$ solution in a glass separatory funnel. The contents were shaken for $2 \mathrm{~min}$ before the $\mathrm{CHCl}_{3}$ was removed. Additional $50 \mathrm{ml}$ aliquots of $\mathrm{CHCl}_{3}$ was added until the $\mathrm{CHCl}_{3}$ removed was colourless-pale yellow. To lower levels of $\mathrm{Os}$, the $\mathrm{CrO}_{3}-\mathrm{H}_{2} \mathrm{SO}_{4}$ solution was heated to approximately $100{ }^{\circ} \mathrm{C}$ and then spurged by bubbling clean $\mathrm{N}_{2}$ gas through the solution for $12 \mathrm{~h}$ (Selby and Creaser, 2003). 


\subsubsection{Osmium separation and purification chemistry}

In this project, Os was separated and purified via solvent extraction and micro-distillation methods (Fig. 2.7; Cohen and Waters, 1996; Birck et al., 1997; Selby and Creaser, 2003 and references therein). Once the Carius tube was opened and prior to the sample-acid mixture thawing, $3 \mathrm{ml}$ of $\mathrm{CHCl}_{3}$ was added. The $\mathrm{CHCl}_{3}$ acts as an organic solvent which extracts $\mathrm{OsO}_{4}$ from the digestion mixture (Cohen and Waters, 1996). Once thawed, the content of the tube was transferred into $50 \mathrm{ml}$ centrifuge tubes, sealed, agitated and then placed in a water bath at room temperature for 15 minutes. This step allowed all the $\mathrm{OsO}_{4}$ to be extracted into the $\mathrm{CHCl}_{3}$ solvent. The mixture was then centrifuged and the $\mathrm{CHCl}_{3}$ phase containing the $\mathrm{OsO}_{4}$ was transferred into a $22 \mathrm{ml}$ glass vial containing $3 \mathrm{ml}$ concentrated $(9 \mathrm{~N}) \mathrm{HBr}$ solution for back extraction. The $\mathrm{HBr}$ solution reduces the $\mathrm{OsO}_{4}$ to $\left[\mathrm{OsBr}_{6}\right]^{2-}$ (Birck et al., 1997). The above steps were repeated twice to ensure all the Os had been extracted before the $\mathrm{HBr}-\mathrm{CHCl}_{3}$ mixture was placed on a rocker overnight. The residual acid-sample mixture was kept for Re extraction (Section 2.3.3).

Using a clean pipette tip, the $\mathrm{CHCl}_{3}$ was removed to waste and the Os-bearing $\mathrm{HBr}$ solution was then transferred to a clean $7 \mathrm{ml}$ Savillex ${ }^{\mathrm{TM}}$ Teflon vial and evaporated to dryness overnight at $80{ }^{\circ} \mathrm{C}$. For inverse aqua-regia digested samples, the $\mathrm{CHCl}_{3}$ phase was at the base of the glass vials. For $\mathrm{CrO}_{3}-\mathrm{H}_{2} \mathrm{SO}_{4}$ digested samples, the $\mathrm{CHCl}_{3}$ phase was located along the sides and towards the top of the glass vial. The dry Os was meticulously transferred using $\sim 50 \mu 19 \mathrm{~N} \mathrm{HBr}$ to the lid of a $5 \mathrm{~mL}$ conical Savillex ${ }^{\mathrm{TM}}$ vials micro-distillation vial (Fig. 2.7) and then dried at $80{ }^{\circ} \mathrm{C} .20 \mu \mathrm{l}$ of $9 \mathrm{~N} \mathrm{HBr}$ was placed directly to the base of the $5 \mathrm{~mL}$ conical Savillex ${ }^{\mathrm{TM}}$ vials vial and the vial turned upside down. Surface tension between the vial and $\mathrm{HBr}$ solution allows the $\mathrm{HBr}$ to be held at the tip of the upside-down vial (Fig 2.7). To the dried $\left[\mathrm{OsBr}_{6}\right]^{2-}$ on the lid, $30 \mu \mathrm{l}$ of $\mathrm{CrO}_{3}-12 \mathrm{~N} \mathrm{H}_{2} \mathrm{SO}_{4}$ solution was added, taking care that no air bubbles developed. As the Os is volatilised by the $\mathrm{CrO}_{3}-12 \mathrm{~N} \mathrm{H}_{2} \mathrm{SO}_{4}$ solution, the $5 \mathrm{~mL}$ conical Savillex ${ }^{\mathrm{TM}}$ vials vial was quickly but carefully sealed onto the lid to prevent the loss of the volatile $\mathrm{OsO}_{4}$. The upside-down vial was then placed on a hot plate $\left(80^{\circ} \mathrm{C}\right)$ for $3-4$ hours, taking extreme care not to disturb the $\mathrm{HBr}$ being held by surface tension at the top of the vial. After the distillation process, the vial was removed from the lid, turned back to its normal position, and the Osbearing $\mathrm{HBr}$ was dried down at $60{ }^{\circ} \mathrm{C}$ until only $\sim 1 \mu \mathrm{l}$ (enough for loading on the filament of a mass spectrometer) remained. The content of the lid was discarded. 


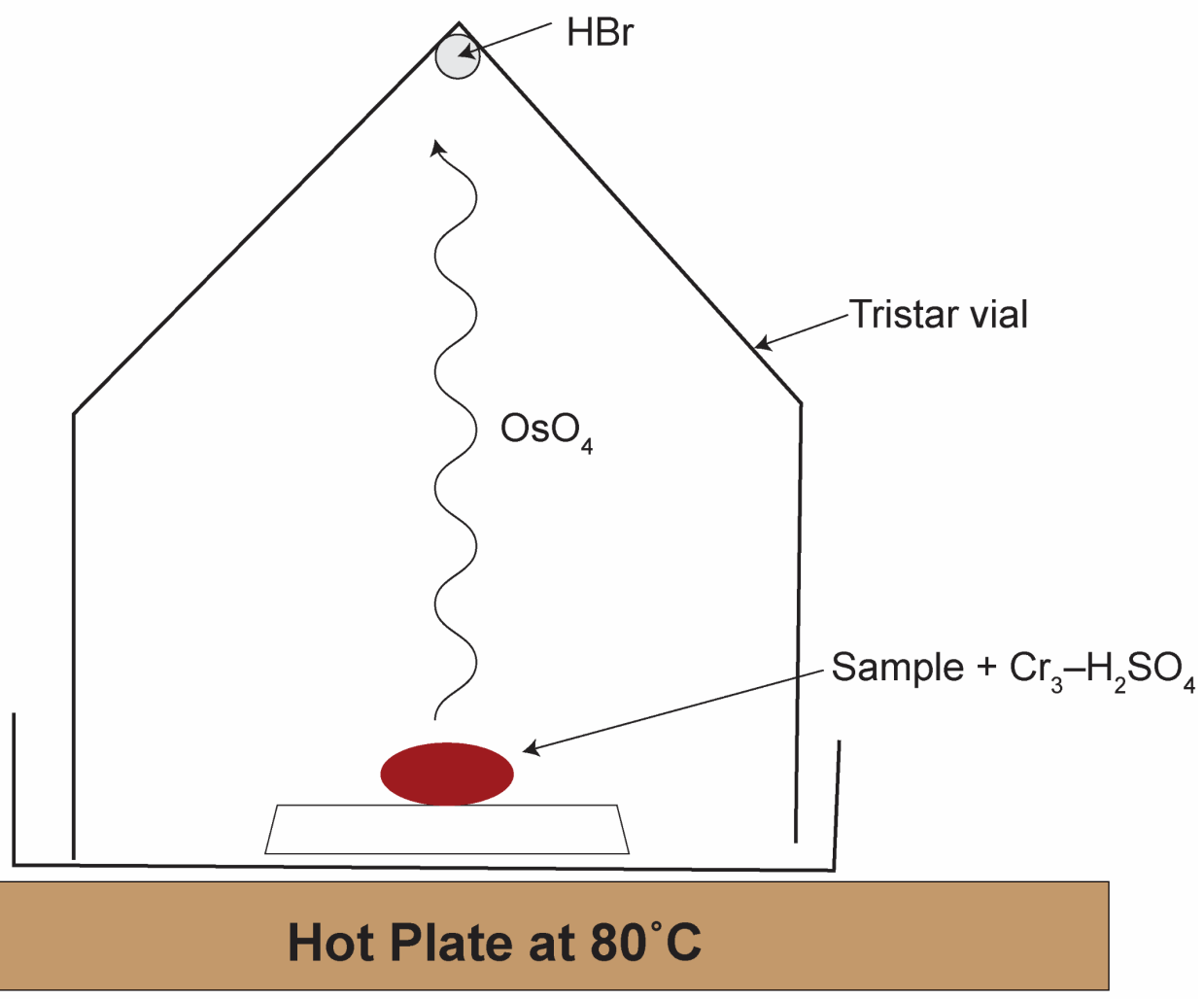

Fig. 2.7. Schematic illustration of Os micro-distillation based on the method of Birck et al. (1997).

\subsubsection{Rhenium separation chemistry}

Following Os solvent extraction, $2 \mathrm{ml}$ of the Re-bearing acid-sample mixture was evaporated to dryness at $100{ }^{\circ} \mathrm{C}$ in a pre-cleaned glass vial. $10 \mathrm{ml} 5 \mathrm{~N} \mathrm{NaOH}$ solution was then added to the dried sample and left to dissolve for 30 minutes. Once the sample had dissolved, the $\mathrm{NaOH}-$ sample solution was transferred to a $15 \mathrm{ml}$ centrifuge tube, $10 \mathrm{ml}$ acetone added and the mixture agitated for 10 minutes and then centrifuged for 1 minute. Acetone (now Re bearing) is less dense than $\mathrm{NaOH}$, which allowed it to be transferred to a cleaned glass vial using a pipette, and then evaporated at $60{ }^{\circ} \mathrm{C}$ overnight. To the dried sample cake, $3 \mathrm{ml}$ of $0.2 \mathrm{~N} \mathrm{HNO}_{3}$ was added in readiness for anion exchange chromatography.

The Re anion exchange chromatography was carried out using disposable pipettes as columns. The top and tip of these pipettes were cut off and a small piece of quartz wool was placed into the pipette to act as a frit. The pipette was then filled with ultra-pure water and any air bubble that may have formed removed. Eichrom ${ }^{\mathrm{TM}} 1 \mathrm{X} 8,100-200$ mesh resin was then added to fill the entire graduate column of the pipette. The resin in the column was washed with $4 \mathrm{ml}$ of $8 \mathrm{~N}$ $\mathrm{HNO}_{3}$ and then equilibrated with $4 \mathrm{ml} 0.2 \mathrm{~N} \mathrm{HNO}_{3}$ ready for sample elution. 
The dissolved sample was loaded onto the column using a clean pipette for each sample. The stem walls were then rinsed four times with $0.25 \mathrm{ml}$ of $0.2 \mathrm{~N} \mathrm{HNO}_{3}$ and twice with $1 \mathrm{ml}$ of $0.2 \mathrm{~N}$ $\mathrm{HNO}_{3}$. The resin was then washed twice with $1 \mathrm{ml}$ of $0.2 \mathrm{~N} \mathrm{HCl}$ and twice again with $2 \mathrm{ml}$ of $6 \mathrm{~N} \mathrm{HNO}_{3}$. At this point, the Re fraction was ready for collection in a pre-cleaned Teflon ${ }^{\mathrm{TM}}$ beaker using $4 \mathrm{ml}$ of $6 \mathrm{~N} \mathrm{HNO}_{3}$. Finally, the collected Re was dried on a hot plate at $\sim 80{ }^{\circ} \mathrm{C}$.

\subsection{Isotope dilution in Re-Os analysis}

Isotope dilution analysis is the benchmark method for determination of high precision $\mathrm{Re}$ and Os concentrations (Schoenberg et al., 2000; Nozaki et al., 2012; Dickin, 2018). In this method, an isotopically enriched solution (commonly referred to as spike or tracer solution) with a known concentration of the element with the artificially enriched isotope is added to the sample being analysed, which contains the natural isotope composition of the element (Figs 2.8, 2.9). During sample digestion, the spike and sample isotopes equilibrate, and the resulting mixed isotopic composition is measured and used to calculate the concentration of the element in the sample. A prerequisite for isotope dilution analysis is that the target element should comprise two or more naturally occurring isotopes.

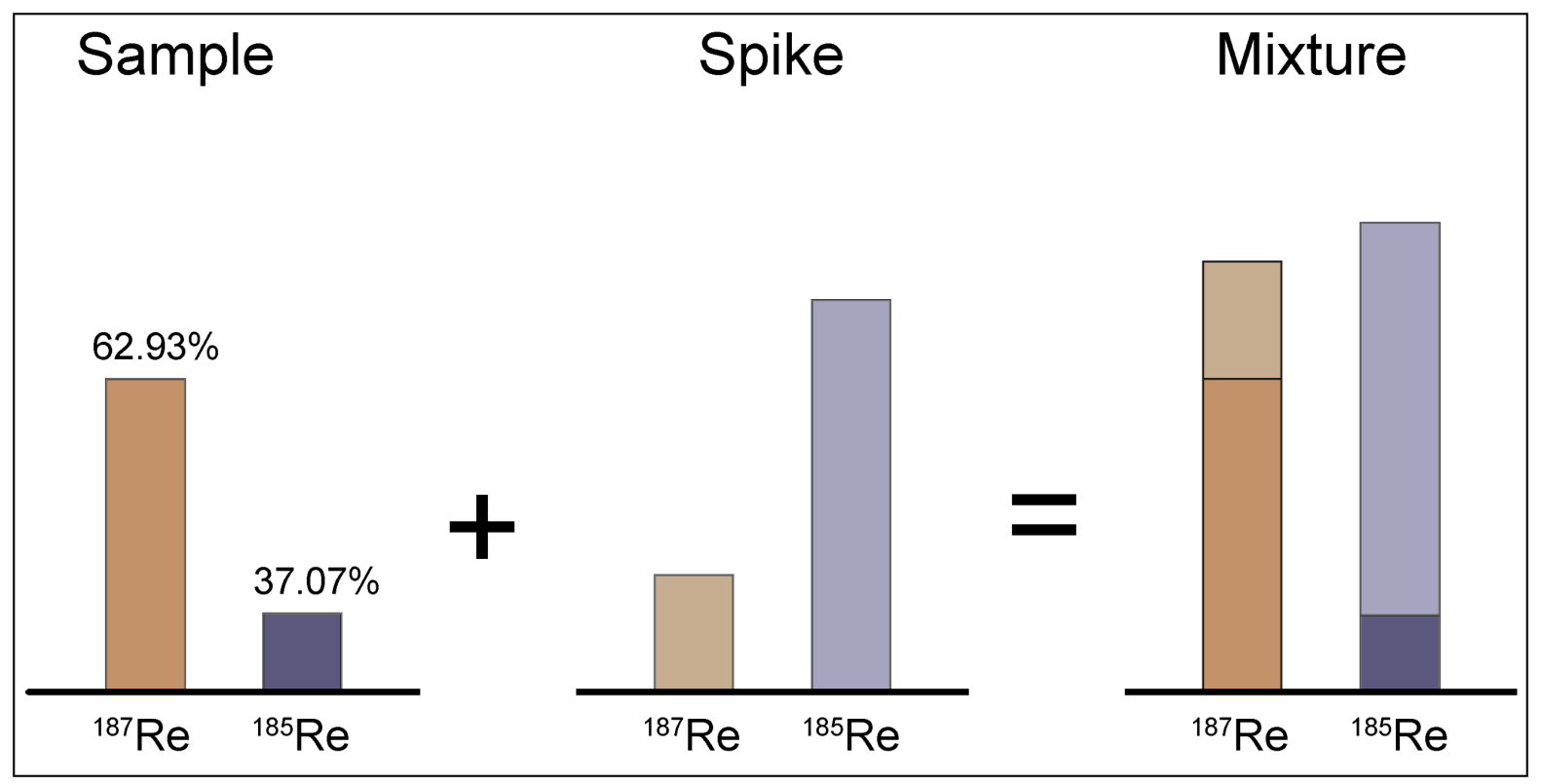

Fig. 2.8. Principles of isotope dilution as shown for Re. The element has two naturally occurring isotopes, ${ }^{185} \mathrm{Re}$ and ${ }^{187} \mathrm{Re}$, with natural abundance of $37.07 \%$ and $62.93 \%$, respectively. In the spike solution, the ${ }^{185} \mathrm{Re}$ isotope is artificially enriched. A known amount of the spike is added to the sample, equilibrated during acid digestion and the ${ }^{187} \mathrm{Re} /{ }^{185} \mathrm{Re}$ isotope ratio measured on a mass spectrometer. 
Taking Re as an example (Fig. 2.8), the Re concentration in the sample can be derived from the measured isotope ratio, $\mathrm{R}_{\mathrm{M}}\left({ }^{187} \mathrm{Re} /{ }^{185} \mathrm{Re}\right)$, which represents a mixture of natural (sample) and spike Re (Patterson et al., 1994; Dickin, 2018):

$$
R_{M}=\frac{M_{N} A 187_{N}+M_{S} A 187_{S}}{M_{N} A 185_{N}+M_{S} A 185_{S}}
$$

where $\mathrm{M}_{\mathrm{N}}$ and $\mathrm{M}_{\mathrm{S}}$ are the moles of natural Re in the sample and spike Re, respectively; and $\mathrm{A}$ indicates the abundance of the ${ }^{187} \mathrm{Re}$ and ${ }^{185} \mathrm{Re}$ isotopes in the natural and spike solutions.

The number of moles of Re in the sample $\left(\mathrm{M}_{\mathrm{N}}\right)$ is first calculated, given that other terms in the equation are known:

$$
M_{N}=M_{S} \frac{A 187_{S}-R_{M} A 185_{S}}{R_{M} A 185_{N}-A 187_{N}}
$$

The number of moles of an element $=$ molarity $x$ mass. Therefore, the molarity of Re in the sample can be expressed as:

$$
\text { Molarity }_{N}=\text { Molarity }_{S} \times \frac{A 187_{S}-R_{M} A 185_{S}}{R_{M} A 185_{N}-A 187_{N}} \times \frac{w t_{S}}{w t_{N}}
$$

where wts and $\mathrm{wt}_{\mathrm{N}}$ are the measured weights of the spike and sample, respectively.

To obtain the concentration of Re in the sample, the molarity is multiplied by atomic weight:

$$
\operatorname{Conc}_{N}=A t \times w t_{N} \times \frac{A 187_{S}-R_{M} A 185_{S}}{R_{M} A 185_{N}-A 187_{N}} \times \frac{w t_{S}}{w t_{N}} \times \frac{\text { Conc }_{S}}{A t \times w t_{S}}
$$

where At $=$ the atomic weight and conc $=$ concentration.

In the case of Os, the spike used is enriched in the stable ${ }^{190}$ Os isotope (Fig. 2.9; Schoenberg et al., 2000; Tessalina et al., 2015). The Os concentration in the sample is then calculated relative to any of the other naturally occurring isotopes of Os. For example, in this thesis the ${ }^{190} \mathrm{Os} /{ }^{188} \mathrm{Os}$ ratio was measured to obtain $\mathrm{R}_{\mathrm{M}}$, which is expressed as: 


$$
R_{M}=\frac{M_{N} A 190_{N}+M_{S} A 190_{S}}{M_{N} A 188_{N}+M_{S} A 188_{S}}
$$

where all terms are similar to those in Eq. 2.1. Subsequent derivation of the Os concentrations follows similar steps as those described above for Re (Dickin, 2018).

Isotope dilution is the preferred method for determining Re and Os concentrations because of several key advantages over other conventional techniques such as external calibration or standard addition. The main advantage of this method is that during the entire analytical procedure, quantitative recovery of the analyte is not necessary once the sample and spike have equilibrated. To make full use of this advantage, the spike is added as early as possible in the analytical procedure, before sample digestion. The second advantage of the isotope dilution method is that it can provide precise and accurate data even for samples with very low concentrations (ppt level) by limiting errors introduced by instrument instability or signal drift, isobaric interferences and matrix effects. Further, isotope dilution is an absolute method that only requires measurement of isotope ratios, unlike other techniques which rely on relative measurements such as standard calibrations of an instrument (Patterson et al., 1994). 


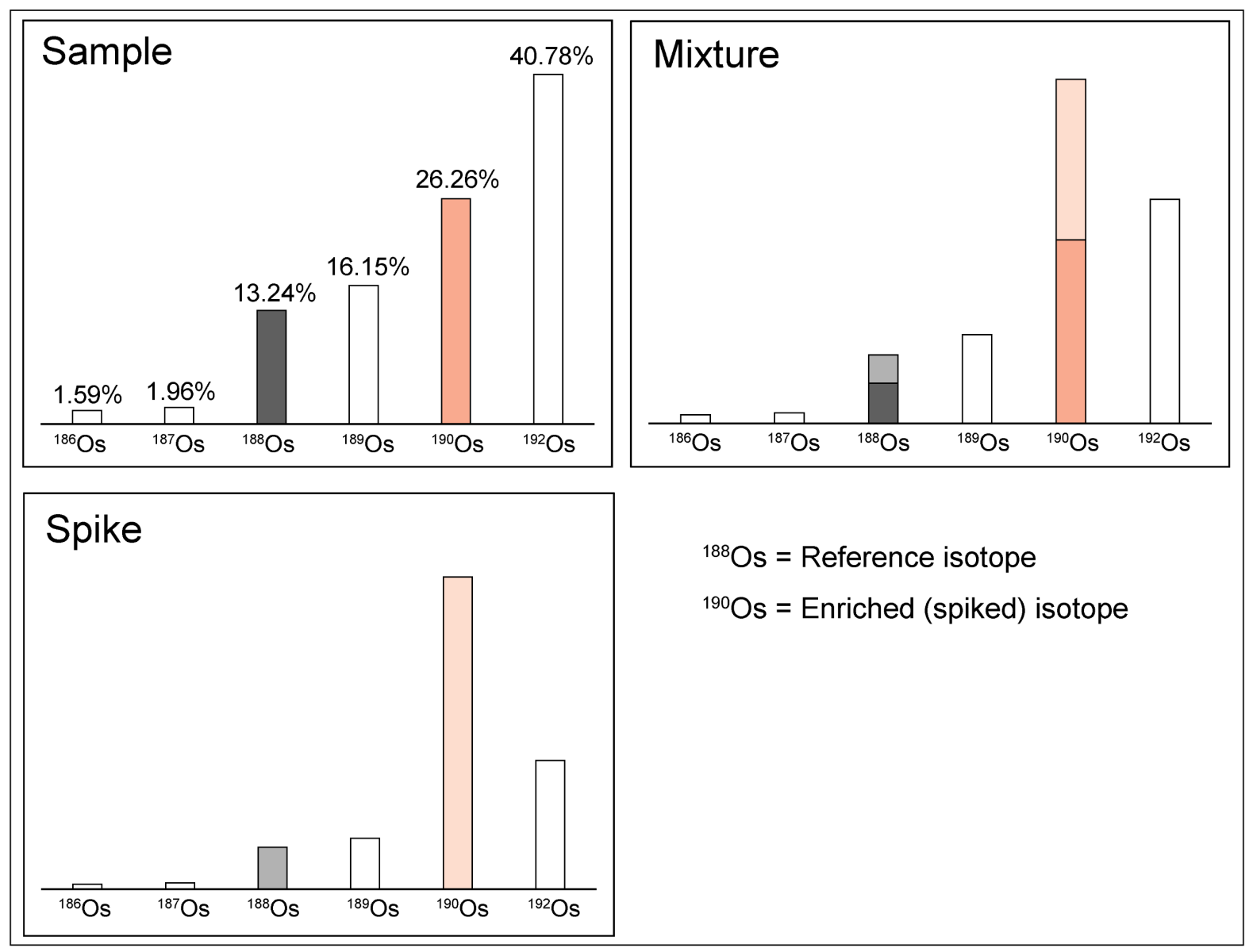

Fig. 2.9. Principles of isotope dilution as shown for Os. Six of the seven (with abundance $>1 \%$ ) naturally occurring isotopes of the element are shown here. In the spike solution, the ${ }^{190} \mathrm{Os}$ isotope is artificially enriched. The ${ }^{188} \mathrm{Os}$ and ${ }^{190} \mathrm{Os}$ are differentiated by the darker and lighter grey and orange colours, respectively, as these are measured for isotope dilution calculation.

A significant drawback for the isotope dilution method is the difficulty in estimating error propagation which changes with spike-sample ratio, becoming exponentially large as the sample-spike ratio approaches the natural ratio (under-spiking) or spike ratio (over-spiking; Fig. 2.10). There is a theoretical optimum when the sample-spike ratio achieves the best precision in the isotopic measurement. This optimum occurs when the measured ratio ( $\left.\mathrm{R}_{\mathrm{M}}\right)$ is equal to the square root of the product of the natural $\left(\mathrm{R}_{\mathrm{N}}\right)$ and spike $\left(\mathrm{Rs}_{\mathrm{S}}\right)$ isotope ratios:

$$
R_{M}=\sqrt{R_{N} \times R_{S}}
$$




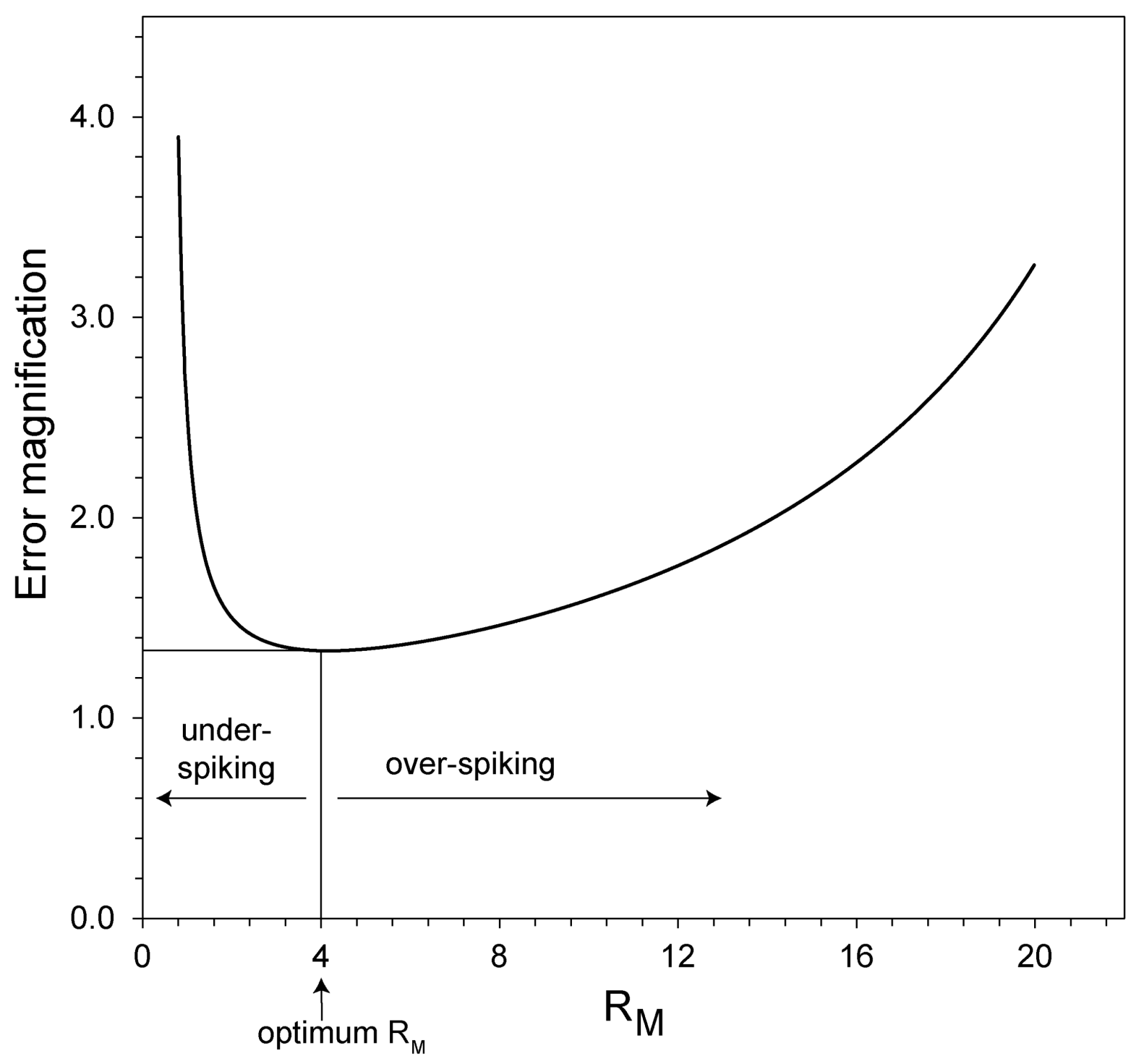

Fig. 2.10. Illustration of error magnification in isotope dilution analysis, using an example from determinations of Re concentrations in rock samples at VUW. This error magnification model was obtained by running Monte Carlo simulations of randomized distribution (uniform) of $\mathrm{R}_{\mathrm{M}}$ values between 0.6 (under-spiked, $\mathrm{R}_{\mathrm{M}} \approx \mathrm{R}_{\mathrm{N}}$ ) and 28.5 (over-spiked, $\mathrm{R}_{\mathrm{M}} \approx \mathrm{R}_{\mathrm{S}}$ ) in Excel using the XLSTAT statistical software. The error magnification is not symmetrical about the optimum, with under-spiking exhibiting a steeper increase in magnification of errors.

To ensure the spike-sample ratio is optimised, initial testing of samples is undertaken to determine the concentration levels of the element of interest and hence guide the amount spike to use. For Re-Os analysis, only Re is typically tested because sample digestion and separation can easily be achieved through conventional Teflon open-beaker methods and column chromatography, respectively, compared to Os which requires the more specialised Carius tube digestion technique and more elaborate separation steps. 
These Re tests were undertaken in the Geochemical Laboratory at Victoria University of Wellington (VUW), on powdered rock samples using $\mathrm{HCl}-\mathrm{HNO}_{3}$ open-beaker dissolution methods and anion exchange chromatography (Appendix 2) following previously published protocols (e.g., Matusiewicz, 2003). The results were used to infer the approximate concentration range for Os in the samples based on the assumption that both elements have similar chelation ability in organic-rich sedimentary rocks. The Re data were also used to select and prioritise samples for analysis at Durham University.

\subsection{Mass spectrometric techniques for Re-Os isotope analysis}

The development of the Re-Os isotopic system as a geochemical tool was initially limited by lack of analytical techniques capable of precisely measuring the low abundances of Re and Os in geological samples. The high ionisation potential $(\sim 9 \mathrm{eV})$ and refractory character exhibited by Os meant that most mass spectrometric techniques such as positive thermal ionisation mass spectrometry (Wasserburg et al., 1969), secondary ion mass spectrometry (SIMS; Allègre and Luck, 1980), accelerated mass spectrometry (AMS; Fehn et al., 1986), resonance ionisation mass spectrometry (RIMS; Walker and Fassett, 1986) and inductively coupled plasma mass spectrometry (ICPMS; Russ et al., 1987) suffered from ionisation inefficiencies. The most significant improvement in Re-Os isotope mass spectrometry was the successful development of negative thermal ionisation mass spectrometry (N-TIMS; Fig. 2.11a; Creaser et al., 1991; Völkening et al., 1991). This technique provided significantly improved ionisation efficiencies

and allowed determination of isotopic ratios with a low detection limit (below $10^{-14} \mathrm{~g}$ ) and an analytical precision that is over an order of magnitude greater than the earlier techniques mentioned above (better than $\pm 2 \%$ ). This improvement in sensitivity and precision of Re and Os analysis using N-TIMS has made it the most commonly used technique, especially for geological samples with low concentrations of these elements (Hattori et al., 1998; Birck, 2001; Luguet et al., 2008; Liu and Pearson, 2014). 


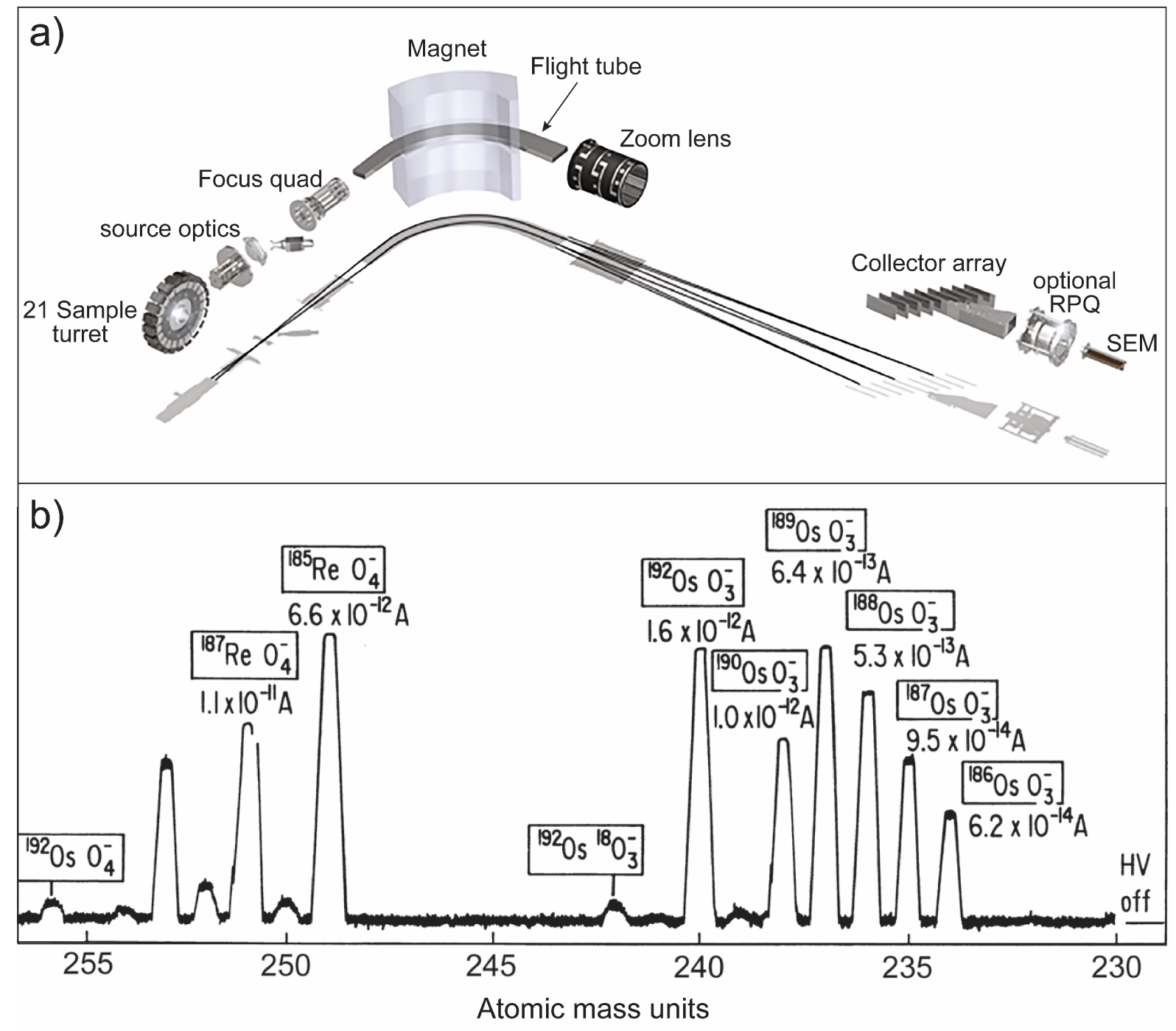

Fig. 2.11. a) Schematic diagram of the Triton negative Thermal Ionisation Mass Spectrometer (NTIMS; Thermo Fisher Scientific Inc., 2018), the instrument used in this study. b) N-TIMS mass spectrum showing the dominant species of $\mathrm{Re}\left(\mathrm{ReO}_{4}{ }^{-}\right)$and $\mathrm{Os}\left(\mathrm{OsO}_{3}{ }^{-}\right)$produced at $770{ }^{\circ} \mathrm{C}$ by a platinum filament loaded with a mixture of $5 \mathrm{ng}$ Os and $3 \mathrm{ng}$ Re. Adapted from Creaser et al. (1991).

In thermal ionization mass spectrometry, a sample is loaded onto a refractory metal filament, which is placed into the ion source of the mass spectrometer. The ion source is then evacuated to create a vacuum and an electric current is applied to the filament to heat it to a point where simultaneous vaporization and ionization of the element of interest occurs (Wieser and Brand, 1999; Birck, 2001; Becker, 2012). The probability that the element of interest is desorbed as negative ions from the hot filament surface depends on the temperature of the filament $(\mathrm{T})$, electron affinity of the element (EA) and work function of the filament (W; Wieser and Brand, 1999). This relationship is described by the Langmuir-Saha equation: 


$$
\frac{N^{-}}{N^{o}} \propto \exp \left[\frac{(E A-W)}{k T}\right]
$$

where $\mathrm{N}^{-}$and $\mathrm{N}^{\circ}$ are the numbers of negative ions and neutral species desorbed from the filament, respectively and $\mathrm{k}$ is the Boltzmann's constant.

If the ionisation energy of the element is low compared to the work function of the filament, a small proportion of the element's atoms are ionised (ionisation efficiencies of $2-6 \%$ for Os and $>20 \%$ for Re; Creaser et al., 1991; Gill, 1997; Wieser and Brand, 1999). Conventionally, this produces positive ions that are then accelerated into the mass spectrometer. In N-TIMS, negative ions are produced due to the reversed polarities of the magnet and ion-acceleration voltage power supplies (Creaser et al., 1991). Rhenium is loaded onto nickel filaments and ionised as the $\mathrm{ReO}_{4}{ }^{-}$species. Osmium is loaded onto platinum filaments and ionised as the $\mathrm{OsO}_{3}{ }^{-}$species (Fig. 2.11b). The filaments are coated with an activator [barium salt; typically $\mathrm{Ba}\left(\mathrm{NO}_{3}\right)_{2}$ or $\left.\mathrm{Ba}(\mathrm{OH})_{2}\right]$ to lower its electron work function and to increase the emission of negative ions (Creaser et al., 1991). The intensities of the beams can be increased by bleeding small quantities of high purity oxygen into the ion source (which is otherwise at vacuum) to promote the formation of $\mathrm{ReO}_{4}{ }^{-}$and $\mathrm{OsO}_{3}{ }^{-}$oxide species. As the $\mathrm{Re}$ and Os analyses are measured as oxides, the effects of variable isobaric interferences involving the heavier oxygen isotopes $\left({ }^{17} \mathrm{O}\right.$ and $\left.{ }^{18} \mathrm{O}\right)$ should be monitored and corrected. For instance, the interference of ${ }^{188} \mathrm{Os}^{16} \mathrm{O}_{2}{ }^{17} \mathrm{O}^{-}$on ${ }^{189} \mathrm{Oss}^{16} \mathrm{O}_{3}{ }^{-}$ion is monitored at mass 239 (e.g., Liu et al., 1997; Nanne et al., 2017).

In this study, the nickel and platinum filaments were first degassed in air for about 30-60 minutes before sample loading. Approximately $1 \mu 1$ of a sample was then loaded onto the filament, coated with a $\mathrm{Ba}\left(\mathrm{NO}_{3}\right)_{2}$ activator and then placed in the ion source of the mass spectrometer. The source was then evacuated for at least $4 \mathrm{~h}$ prior to isotopic measurements. Intense $\mathrm{Re}$ and $\mathrm{Os}$ ion beams were generally observed at temperatures below $800{ }^{\circ} \mathrm{C}$, low enough to prevent emission of electrons. For most of the samples, Re ion beams were large enough to be measured by Faraday cup detectors in static mode (using a multiple detector array). The Faraday cup detectors are highly regarded for accuracy because the number of ions impacting are directly proportional to the measured current (Gill, 1997; Busch, 2011). However, samples with very low Re abundance (low ion beams) were measured on the more sensitive secondary electron multiplier (SEM) detector, in peak-hopping mode (using a single 
detector; e.g., Busch, 2011). All Os measurements were made on the SEM. To ensure highprecision of the data, several isotopic measurements were acquired (100 runs for Re and 50 runs for Os) and fluctuations in the data averaged out. Further, the response of the instrument and quality of the data was monitored by running in-house standards [DROsS for Os (Nowell et al., 2008) and Re-Std for Re (Selby, 2007)]. Procedural blanks were also run at every analytical session.

\subsection{Mass-dependent fractionation in N-TIMS}

On the N-TIMS, instrumental mass fractionation occurs in the ion source during sample evaporation and ionization. The evaporation process favours the removal of lighter isotopes, relative to the heavier ones, due to differences in the strength of chemical bonds (Andreasen and Sharma, 2009; Dickin, 2018). With time, the sample remaining in the filament becomes depleted in the lighter isotopes and produces heavier isotopic ratios, a phenomenon termed 'reservoir effect' (Andreasen and Sharma, 2009). A number of empirical (linear, power and exponential) and theoretical (Rayleigh) laws have been developed to correct for this mass fractionation (Russell et al., 1978; Suzuki et al., 2004; Andreasen and Sharma, 2009). In these laws, an assumption is made that sample evaporation only occurs from a single, homogenous area on the filament. However, this is not always the case since mass fractionation is dependent on temperature, which may not be even across a sample-laden filament (Hart and Zindler, 1989). The linear and power laws are indistinguishable because both assume that mass fractionation is directly proportional to mass difference only and not to the absolute masses of the evaporating species (Dickin, 2018). Mass fractionation, however, varies inversely with the absolute masses of the species, and is therefore more severe for isotope analysis involving elements with lighter masses such as $\mathrm{Ca}$. The exponential law remedies this problem by introducing a fractionation factor that is dependent on the mass of the evaporating species (Russell et al., 1978). The linear law, which was used in this thesis, is normally sufficient to correct for mass fractionation in heavier isotopes such as those of Re and Os (Dickin, 2018).

For elements with two or more non-radiogenic (stable) isotopes, mass fractionation can be corrected by adjusting the measured ratio of interest to a reference ratio comprising two stable isotopes with a known or "true" ratio, a technique known as internal normalization (Moore et al., 1974; Roy-Barman et al., 1998; Platzner, 1999). In the case of Os, the fractionation of 
${ }^{187} \mathrm{Os} /{ }^{188} \mathrm{Os}$ ratio is corrected using the ${ }^{192} \mathrm{Os} /{ }^{188} \mathrm{Os}$ ratio, which has a constant value of 3.08271 (Platzner, 1999). Using the linear law, the deviation of the measured ${ }^{192} \mathrm{Os} /{ }^{188} \mathrm{Os}$ ratio from the "true" value (3.08271) at each point through the analysis is divided by the difference in atomic masses of the two isotopes $\left(\Delta_{\text {mass }}=4.006\right)$ to yield the coefficient of fractionation per mass unit (f) as shown in equation 2.8 .

$$
f=\frac{\frac{\left({ }^{192} O s /{ }^{188} O s\right)_{\text {measured }}}{3.08271}-1}{\Delta_{\text {mass }}}
$$

This coefficient of fractionation is then used to correct the measured ${ }^{187} \mathrm{Os} /{ }^{188} \mathrm{Os}$ ratio, for which $\Delta_{\text {mass }}=1$ :

$$
\left(\frac{{ }^{187} O s}{{ }^{188} O s}\right)_{\text {true }}=\left(\frac{{ }^{187} O s}{{ }^{188} O s}\right)_{\text {measured }} \times\left(1+f \Delta_{\text {mass }}\right)
$$

The internal normalization technique does not work for Re because it only has two isotopes, and one of the isotopes $\left({ }^{185} \mathrm{Re}\right)$ is introduced as a spike. For N-TIMS, Re mass fractionation can be resolved in two ways: 1) making several measurements of a standard and assuming that the obtained average natural ${ }^{187} \mathrm{Re} /{ }^{185} \mathrm{Re}$ ratio will be the same for sample analyses, or 2 ) evaporation of the entire sample in the filament and the signal from each isotope is integrated (Suzuki et al., 2004). The latter effectively eliminates the effects of mass fractionation during the evaporation process, but it only works for very small loads that can be pragmatically exhausted on a filament. In this study, mass fractionation of the Re isotopes was resolved by averaging out several measurements of the Durham University in-house Re standard (Re-Std; Selby, 2007).

\subsection{Linear regression in Re-Os isochron geochronology}

The Re-Os geochronology results of this thesis are presented as isochrons and thus a brief description of the rationale behind data regression and interpretation is warranted. When the radiogenic Os isotope ratio $\left({ }^{187} \mathrm{Os} /{ }^{188} \mathrm{Os}\right)$ is plotted against the parent-daughter ratio $\left({ }^{187} \mathrm{Re} /{ }^{188} \mathrm{Os}\right)$ for samples deposited at the same time (isochronous) and with the same initial ${ }^{187} \mathrm{Os} /{ }^{188} \mathrm{Os}$ at time of deposition, the data points should ideally define a straight line (isochron) defined by equation 2.10 . 


$$
\left(\frac{{ }^{187} O s}{{ }^{188} O s}\right)_{\text {present }}=\left(\frac{{ }^{187} R e}{{ }^{188} O s}\right) \times\left(e^{\lambda t}-1\right)+\left(\frac{{ }^{187} O s}{{ }^{188} O s}\right)_{\text {initial }}
$$

where $t$ is the age of a sample in years and $\lambda$ is the ${ }^{187} \mathrm{Re}$ decay constant. The slope of this line $\left(\mathrm{e}^{\lambda \mathrm{t}}-1\right)$ is then used to determine the age of a sample, whereby older samples have a steeper slope because they have undergone more radioactive decay. The y-intercept is the initial ${ }^{187} \mathrm{Os} /{ }^{188} \mathrm{Os}\left(\mathrm{Os}_{\mathrm{i}}\right)$ at the time of formation, which provides information about the origin or processes of formation of a sample.

As both radiogenic and parent-daughter ratios are measured experimentally, analytical errors (uncertainties) must be considered in isochron regression models together with those that arise from geological factors such as an open system behaviour and variation in Osi values. Potential sources of analytical error include sample weighing, spike calibration, blank Re and Os abundances and isotopic composition, and external reproducibility of measurements (as determined by replicate analyses of the Re and Os standards). These errors cause scatter from the line of best-fit, increasing the uncertainty of an isochron age. One mechanism for assessing the size of these errors is through the error correlation function (rho), which can vary from 0 to 1. A rho value of 0 indicates that there is no correlation between the data in the $\mathrm{x}$ and $\mathrm{y}$ axes, ${ }^{187} \mathrm{Re} /{ }^{188} \mathrm{Os}$ and ${ }^{187} \mathrm{Os} /{ }^{188} \mathrm{Os}$ ratios, respectively. A rho value of 1 indicates a perfect linear correlation between ${ }^{187} \mathrm{Re} /{ }^{188} \mathrm{Os}$ and ${ }^{187} \mathrm{Os} /{ }^{188} \mathrm{Os}$ ratios.

Alternatively, the effects of the analytical and model errors on the Re-Os geochronological results can be quantified through the size of their mean squared weighted deviation (MSWD). MSWD is obtained by summing the squares of the deviation of each point from the isochron line (York, 1968) and dividing it by the degrees of freedom. MSWD will tend to be near unity when the observed deviation from the isochron line is only caused by assigned analytical errors. MSWD values $>>1$ generally indicate either an underestimation of the analytical errors or presence of geological scatter. MSWD values $<<1$ normally indicate an overestimation of the analytical errors (Wendt and Carl, 1991; Ludwig, 2012). To obtain isochrons that yield meaningful ages, the MSWD value is generally required to be $<2.5$ (Wendt and Carl, 1991). A low MSWD, however, does not always indicate the obtained isochron age is precise or accurate because large uncertainties in individual measurements can increase the probability of fit, which lowers the MSWD value. 
In this thesis, the Re-Os geochronological data were regressed using the Microsoft Excel AddIn Isoplot version 4.15 (Ludwig, 2012). The Isoplot program employs three different models to account for propagation of errors and scatter in data, based on the probability of how well the data fit the regression line. If the probability of fit is such that the scatter in the data from the isochron line can be accounted for by individually assigned analytical errors, the program produces a Model 1 age using York's algorithm (York, 1968). If the probability of fit is low $(<0.15)$, the program rejects the Model 1 regression and calculates a Model 3 age, which assumes that scatter in the data is attributable to a combination of the assigned analytical errors and unknown, but normally distributed, geological errors that cause variation in the Osi ratios (Ludwig, 2012). The Isoplot program also offers a Model 2 age which assigns equal weights and zero error correlations to the samples, but this is rarely justified for classical isochrons such as the $\mathrm{Rb}-\mathrm{Sr}, \mathrm{Sm}-\mathrm{Nd}, \mathrm{U}-\mathrm{Pb}$ and Re-Os. A new approach that propagates uncertainties in a consistent manner regardless of the probability of fit has recently been established (https://isochron-beta1.shinyapps.io/isochron/; Li et al., 2019). This new method uses the Monte Carlo sampling for error propagation, giving more realistic estimates of uncertainties, and was therefore applied to the data presented in Chapters 3 and 4.

\subsection{Source rock characterization}

Rock-Eval pyrolysis is used to characterize the type (or quality) and maturity of organic matter (kerogen) in a source rock, which would then provide information on the potential of the rock to produce hydrocarbons. This method involves programmed heating of a small rock sample $(\sim 100 \mathrm{mg})$ in a pyrolysis oven under an inert atmosphere to quantitatively and selectively evaluate (1) the free hydrocarbons present in the sample and (2) the compounds containing hydrocarbons and oxygen $\left(\mathrm{CO}_{2}\right)$ which are volatilized during the cracking of the nonextractable organic matter in the sample (Espitalié et al., 1977; Tissot and Welte, 1984; Peters, 1986).

In summary, Rock-Eval pyrolysis produces four basic parameters (Fig. 2.12; Tissot and Welte, 1984): 
$\mathrm{S} 1=$ the amount of free hydrocarbons present in a sample. It is measured in milligrams of hydrocarbon per gram of rock. If $\mathrm{S} 1$ is greater than $1 \mathrm{mg} \mathrm{HC} / \mathrm{g}$ rock, it likely indicates an oil show. S1 normally increases with depth due to increasing maturity of the source rock. Contamination of samples by drilling fluids and mud should be properly assessed as this can lead to abnormally high S1 values.

$\mathrm{S} 2$ = the amount of hydrocarbons generated through thermal cracking of non-volatile organic matter. This parameter indicates the quantity of hydrocarbons that the rock has the potential of generating when buried to the right temperature and pressure. S2 normally decreases with depths $>1 \mathrm{~km}$.

$\mathrm{S} 3=$ the amount of carbon dioxide (in milligrams $\mathrm{CO}_{2}$ per gram of rock) produced during pyrolysis of the organic matter. This parameter provides an indication of the amount of oxygen in the kerogen and is used to calculate the oxygen index. Abnormally high S3 values may, however, result from contamination of samples or presence of high concentrations of carbonates that break down at lower temperatures than $390^{\circ} \mathrm{C}$.

$\mathrm{T}_{\max }=$ the temperature at which the maximum release of hydrocarbons from cracking of kerogen occurs during pyrolysis (top of S2 peak). This parameter provides an indication of the stage of kerogen maturation.

Rock-Eval pyrolysis can also be used to determine the TOC of a sample. This is done by completely oxidizing (at $600^{\circ} \mathrm{C}$ ) the organic matter remaining in the sample after pyrolysis, often termed residual organic carbon. The TOC is then calculated by adding the residual organic carbon to the pyrolyzed organic carbon. Units for the TOC are usually given as wt \% organic carbon per weight of dry rock 


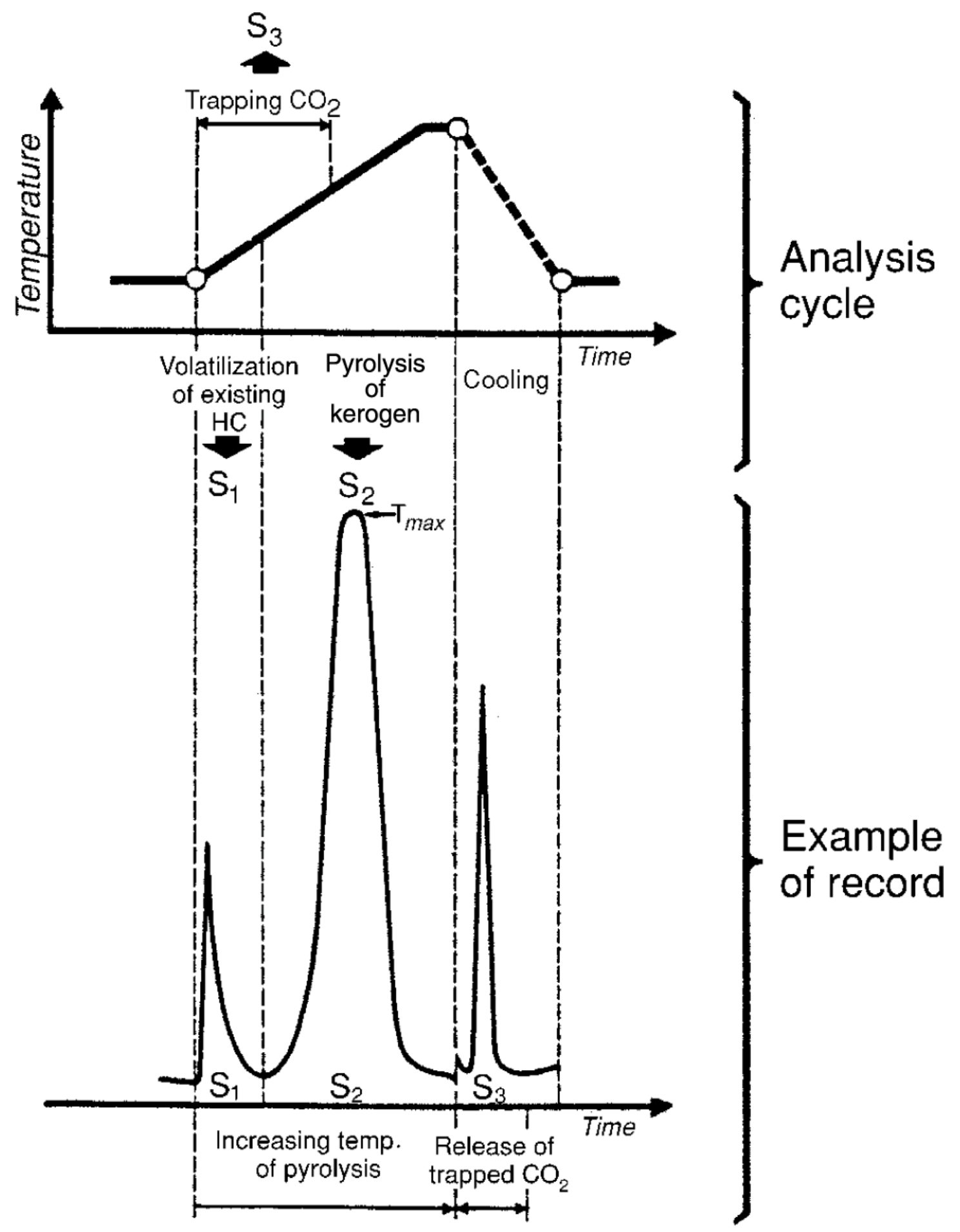

Fig. 2.12. Typical Rock-Eval pyrogram showing cycle of analysis and the corresponding recording (Tissot and Welte, 1984).

\subsubsection{Source rock generative potential}

The TOC content is a measure of the total amount of organic matter present in a source rock and hence, indirectly, its possible petroleum potential. The minimum acceptable TOC content 
for clastic type rocks indicating good source potential is $1.0 \mathrm{wt} . \%$. However, TOC may not always provide a good indication of source rock potential because measurements may include inert carbon that has little or no generative potential (Peters, 1986). Higher TOC values may also not give a good guide to petroleum potential and may not indicate the proportion of gas to oil likely to be generated. This is because the carbon in kerogen is distributed between a number of major chemical component groups which have different carbon contents and varying petroleum potential, depending upon the biomacromolecules from which they are derived. The Rock-Eval S2 parameter is therefore considered a better indicator of generative potential of source rocks, with rocks having S2 greater than 5 and $10 \mathrm{mg} \mathrm{HC} / \mathrm{g}$ rock considered as having good and very good source rock potential, respectively (Peters, 1986). A plot of TOC versus S2 diagram (Fig. 2.13) has been developed to give a visual representation of the quantity of organic matter present and its associated hydrocarbon potential.

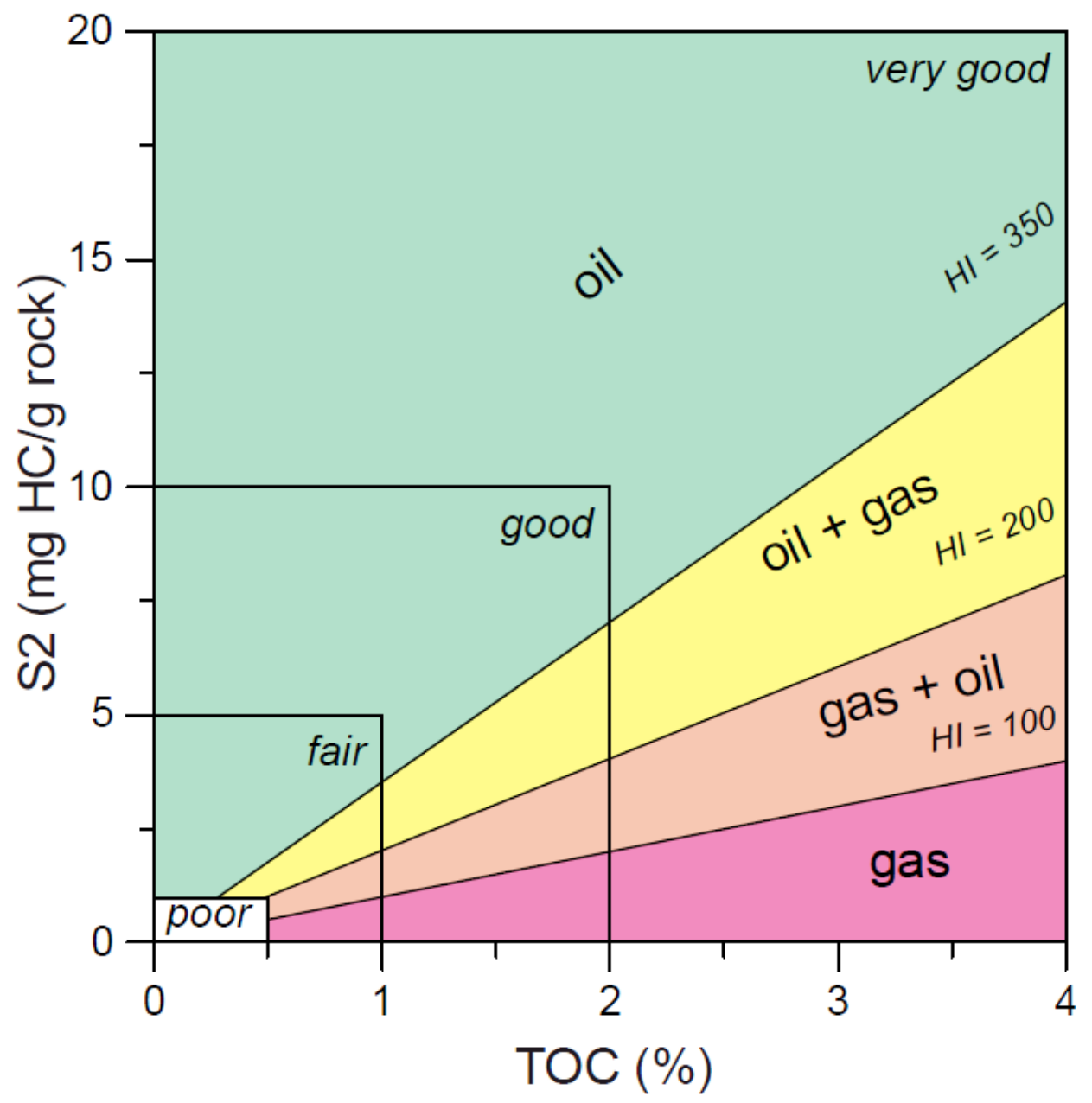

Fig. 2.13. A plot of TOC versus $\mathrm{S} 2$ showing various classifications of hydrocarbon generative potential of a source rock (Peters, 1986; p:IGI programme). 


\subsubsection{Organic matter type}

The type of organic matter present in kerogen determines the amount of hydrocarbons that can be generated by a source rock and also has a major influence relative proportions of oil and gas. Hydrogen index (HI), which represents the S2 parameter normalised to the TOC content $(\mathrm{HI}=[100 \times \mathrm{S} 2] / \mathrm{TOC})$, can be used as a guide to whether a source rock is likely to be oil or gas prone. HI values decrease once hydrocarbon generation commences. Therefore, the total hydrocarbon potential is best measured for samples approaching but not yet within the oil window. Coals and Type III kerogens can present problems when interpreting Rock-Eval data. The HI values appear to increase initially as maturity increases, but subsequently decline with the onset of significant hydrocarbon generation as shown in Fig. 2.14.

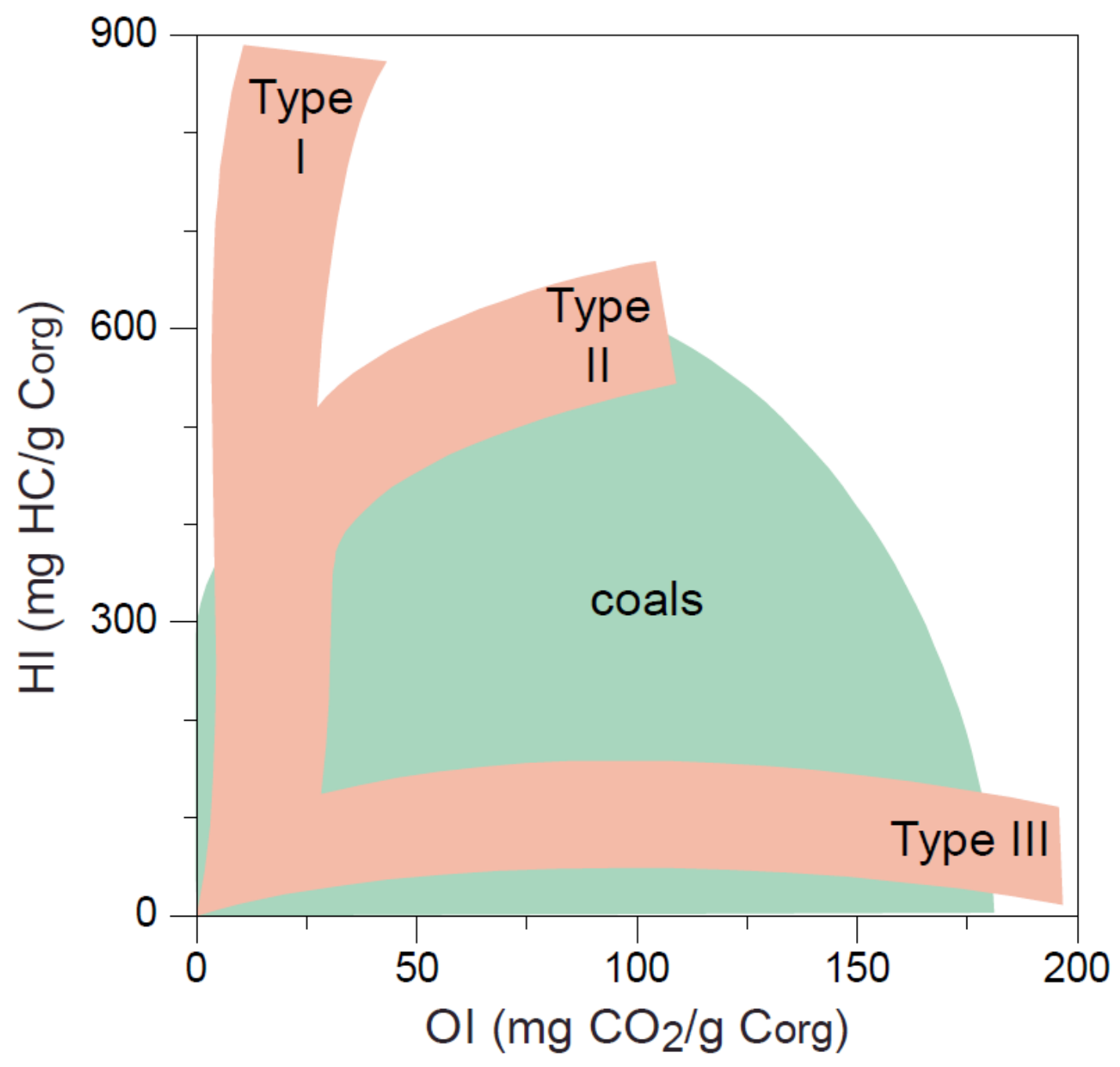

Fig. 2.14. Modified Van Krevelen diagram with indications for diagenetic evolutionary pathways for type I, II and III organic matter (Espitalié et al., 1977; p:IGI programme). 
Although HI changes with increasing maturity once hydrocarbon generation begins, the type of kerogen present in a sample can still be estimated by Rock-Eval pyrolysis using a combination of the S2 and S3 parameters. The S3 parameter can be normalised to TOC to give the Oxygen Index (OI) which can then be plotted against HI to estimate the type of kerogen present using a modified Van Krevelen plot as shown in Fig. 2.14. Kerogens are classified into three main groups: Type I, consisting of mainly algal and amorphous organic matter and highly likely to generate oil; Type II, mixed terrestrial and marine organic matter that can generate waxy oil; and Type III, woody terrestrial organic matter that typically generates gas.

\subsubsection{Thermal maturity}

The thermal maturity of a source rock sample can be estimated by the temperature at which the rate of $\mathrm{S} 2$ pyrolysate generation reaches a maximum $\left(\mathrm{T}_{\max }\right)$. This parameter represents the thermal energy required to break the most abundant chemical bonds in the organic matter associated with the generation of the S2 pyrolysate. The more mature a sample containing a particular kerogen, the higher its $\mathrm{T}_{\max }$ value. However, $\mathrm{T}_{\max }$ is influenced by kerogen type. For instance, an immature type I marine kerogen in which significant amounts of weak C-S bonds are present will have a very low $\mathrm{T}_{\max }$ value compared to a gas-prone Type III kerogen with no such weak bonds. $T_{\max }$ can be used to determine absolute maturities where kerogen composition is uniform and calibration against another maturity parameter like vitrinite reflectance is possible. 


\section{RE-OS GEOCHRONOLOGY AND ISOTOPE SYSTEMATICS,}

AND ORGANIC AND SULFUR GEOCHEMISTRY OF THE Middle-late Paleocene WaipaWa Formation, NeW ZEALAND: INSIGHTS INTO EARLY PALEOGENE SEAWATER OS ISOTOPE COMPOSITION

\section{Preface}

This chapter has been published in the journal Chemical Geology, 536, 119473, 2020, coauthored by Monica Handler (MH, VUW supervisor), Sebastian Naeher (SN, GNS Science cosupervisor), Dave Selby (DS, Durham University), Christopher Hollis (CH, GNS Science) and Richard Sykes (RS, GNS Science).

Selected samples for this study were collected by myself and SN from GNS Science, Lower Hutt and the National Core Store, Featherston, New Zealand, in consultation with RS and CH. All sample preparations, laboratory analyses, data reduction and interpretations, and manuscript preparation were undertaken fully by myself with comments and suggestions from all coauthors. MH, SN, DS and technicians at VUW, GNS Science and Durham University assisted in setting up laboratory protocols and training on instrumentation and data reduction. Sulfur analyses were undertaken by CRL Energy Ltd. Additional unpublished bulk geochemical data were obtained from $R S$ and the GNS Science source rock extracts database. 


\subsection{Introduction}

Marine organic-rich sediments are important archives of past climatic, oceanographic and geodynamic events. The Waipawa Formation is one such organic-rich sedimentary unit that has attracted considerable attention due to its significance for the early Paleogene climate reconstructions of the Southwest Pacific (e.g., Killops et al., 2000; Hollis et al., 2012; 2014; Hines et al., 2019), as well as its potential as a petroleum source rock (Moore, 1988; 1989; Killops et al., 1997; 2000; Schiøler et al., 2010; Field et al., 2018; Naeher et al., 2019). The formation contains a distinctive organofacies, termed the 'Waipawa organofacies', that is dominated by woody phytoclasts despite also showing a high abundance of marine $\mathrm{C}_{30}$ steranes and a heavy carbon isotope $\left(\delta^{13} \mathrm{C}\right)$ signature (values of -24 to $-16 \%$; Killops et al., 2000; Schiøler et al., 2010; Hollis et al., 2014; Field et al., 2018; Naeher et al., 2019). The formation was deposited over a wide geographic extent covering multiple sedimentary basins around New Zealand and reaching as far west as the eastern margin of Tasmania (Fig. 3.1a; Schiøler et al., 2010; Hollis et al., 2014). The widespread deposition of such an unusual organic-rich sediment could only have resulted from a perturbation in Earth's oceanographic and climatic system (Killops et al., 2000; Schiøler et al., 2010; Hollis et al., 2014; Hines et al., 2019). However, it has not been established whether all known occurrences of Waipawa organofacies are the same age. This is because precise dating of the formation through biostratigraphy has so far been complicated by several factors, such as the presence of unconformities, scarcity of agediagnostic fossils, poor age control for dinoflagellate datums, varying geochemical signatures between sections, and lack of reliable paleomagnetic data (Hollis et al., 2014). The Waipawa Formation and correlated units also lack interbedded volcanic beds that could potentially be used for radiometric dating. Available biostratigraphy and magnetostratigraphy from the East Coast Basin, New Zealand, place the timing of deposition of the formation in the late middle to early late Paleocene, spanning the Selandian-Thanetian boundary (Crouch et al., 2014; Hollis et al., 2014). In this chapter, the potential of using the Re-Os isotope system to independently verify this age range and to establish whether correlative units from the other sedimentary basins are coeval is investigated.

Re and Os are organophilic trace metals that become enriched in organic-rich sedimentary rocks (e.g., Ravizza and Turekian, 1989; Cohen et al., 1999; Selby and Creaser, 2003 and references therein). The resulting combination of high and variable Re and Os concentrations 
with variations in ${ }^{187} \mathrm{Re} /{ }^{188} \mathrm{Os}$ positively correlating with ${ }^{187} \mathrm{Os} /{ }^{188} \mathrm{Os}$ values means that the ReOs isotope system can be used to directly obtain precise (in some cases, $<1 \%$ uncertainty) and accurate ages of a wide variety of organic-rich rocks including marine shales (e.g., Ravizza and Turekian, 1989; Cohen et al., 1999; Selby and Creaser, 2005b; Kendall et al., 2006; Selby, 2007; Kendall et al., 2009b; Xu et al., 2009; Georgiev et al., 2011; Georgiev et al., 2017; Tripathy et al., 2018), lacustrine mudstones (Creaser et al., 2008; Poirier and Hillaire-Marcel, 2011; Cumming et al., 2012; Xu et al., 2017), and marine-influenced coals (Tripathy et al., 2015). Previous studies have also shown that post-depositional processes such as greenschist metamorphism and flash pyrolysis associated with igneous intrusions do not appreciably disturb the Re-Os isotope system (Creaser et al., 2002; Kendall et al., 2004; Yang et al., 2009; Rooney et al., 2011; Bertoni et al., 2014). In addition, the Re-Os isotope system remains closed during thermal maturation of organic matter and hydrocarbon generation (Creaser et al., 2002; Selby and Creaser, 2003). Based on these points, the Re-Os system represents an ideal geochronological tool to constrain the age of the Waipawa Formation. However, our current understanding of the exact mechanisms that control enrichment and fractionation of Re and Os in organic-rich sediments remains limited. For instance, despite the strong geochemical affinity that the elements have for organic matter, direct relationships between total organic carbon (TOC) content and Re and Os concentrations are not always evident; some sediments with high TOC may have low Re and Os concentrations and vice versa (Cohen et al., 1999; Kendall et al., 2004; Selby et al., 2009; Rooney et al., 2010). In addition, limited fractionation between $\mathrm{Re}$ and Os, and thus similar ${ }^{187} \mathrm{Re} /{ }^{188} \mathrm{Os}$ and ${ }^{187} \mathrm{Os} /{ }^{188} \mathrm{Os}$, has resulted in production of imprecise ages (e.g., Turgeon et al., 2007; Selby et al., 2009; Rooney et al., 2011; Cumming et al., 2012; Bertoni et al., 2014). To further understand some of the factors controlling uptake and fractionation of Re and Os in sedimentary rocks, the underlying Whangai Formation was included in this study because its organic matter composition and depositional conditions appreciably differ from those of the Waipawa Formation.

In addition to geochronology, the Os isotope composition $\left({ }^{187} \mathrm{Os} /{ }^{188} \mathrm{Os}\right)$ of organic-rich sediments provide useful information about past climatic and oceanographic conditions (Ravizza et al., 2001; Schmitz et al., 2004; Peucker-Ehrenbrink and Ravizza, 2012; Du Vivier et al., 2014; Dickson et al., 2015). The Waipawa Formation was deposited during a prolonged temperature minimum in the mid-Paleocene $(\sim 60-58 \mathrm{Ma})$ prior to the start of progressive warming into the early Eocene. This trend is evident in both high-resolution deep-sea oxygen 
and carbon isotope records (Westerhold et al., 2011; Littler et al., 2014; Barnet et al., 2019) as well as regional sea surface temperatures (58-53 Ma; Bijl et al., 2009; Hollis et al., 2012, 2014). The deposition of the formation also corresponds with the first phase of a positive $\delta^{13} \mathrm{C}$ excursion ( 59-57 Ma; Westerhold et al., 2011; Littler et al., 2014; Barnet et al., 2019), which is interpreted to reflect a time of enhanced carbon burial, either as marine (Corfield and Cartlidge, 1992) or terrestrial organic matter (Kurtz et al., 2003). Shifts in $\delta^{13} \mathrm{C}$ of marine organic-rich rocks have also been linked to changes in the rate of oxidative weathering of organic matter buried in continents (Ravizza, 1993; Ravizza and Esser, 1993; Ravizza et al., 2001; Percival et al., 2016; Them et al., 2017; De Lena et al., 2019). The marine Os isotope record can track globally-averaged variations in continental weathering fluxes because at any point in time, the record reflects the balance between radiogenic Os weathered from the continent $\left({ }^{187} \mathrm{Os} /{ }^{188} \mathrm{Os}=\sim 1.4\right)$ and non-radiogenic Os from hydrothermal activities and extraterrestrial materials $\left({ }^{187} \mathrm{Os} /{ }^{188} \mathrm{Os}=0.12\right.$ ) (Peucker-Ehrenbrink and Ravizza, 2000; Cohen, 2004). The residence time of Os in the water column (10-50 ka range, sometimes $<10 \mathrm{ka})$ is also short enough to respond to short-term variations in input but sufficiently long to allow homogenization of the Os signature in the global ocean (Burton et al., 1999; Cohen, 2004; Du Vivier et al., 2014; 2015; Rooney et al., 2016). The seawater Os record for much of the Paleocene, however, remains poorly resolved (Peucker-Ehrenbrink and Ravizza, 2012). The few available ${ }^{187} \mathrm{Os} /{ }^{188} \mathrm{Os}$ data for this period are also based on analyses of pelagic clay sequences (Pegram and Turekian, 1999) and Fe-Mn crusts (Klemm et al., 2005) which have numerical age models that are not easily correlated with those of marine organic-rich sedimentary rocks because they are based on coarse resolution ichthyolith biostratigraphy and empirical growth rates of hydrogenous cobalt (Peucker-Ehrenbrink and Ravizza, 2012). These analyses are also based on a leaching method that may not have preferentially isolated the hydrogenous component of Os, possibly recording inaccurate seawater Os composition due to the potential presence of detrital Os (Pegram and Turekian, 1999; Peucker-Ehrenbrink and Ravizza, 2012). Nonetheless, the pelagic clay record hints at seawater Os with low ${ }^{187} \mathrm{Os} /{ }^{188} \mathrm{Os}$ values of around $0.26-0.32$ from around 57-61 Ma, but the extent and cause of these nonradiogenic ${ }^{187} \mathrm{Os} /{ }^{188} \mathrm{Os}$ values remain unclear due to the low resolution of the record (Pegram and Turekian, 1999; Peucker-Ehrenbrink and Ravizza, 2012).

In this study, the Re-Os elemental and isotope compositions and bulk pyrolysis and sulfur data for the Waipawa and Whangai formations from the Wairarapa and Hawke's Bay region of the 
East Coast Basin, New Zealand (Fig. 3.1a) are presented. One additional Waipawa Formation sample from the North Slope Basin was included to assess Re-Os abundance and isotopic composition of Waipawa organofacies beyond the East Coast Basin (Fig. 3.1a). These data allow us to constrain the depositional age of the Waipawa Formation and contribute to further understanding of mechanistic controls of Re-Os systematics in organic-rich sedimentary rocks. In addition, the Os isotope data of the Waipawa and Whangai formations are included in a compilation of initial ${ }^{187} \mathrm{Os} /{ }^{188} \mathrm{Os}(\mathrm{Osi})$ ratios for sedimentary rocks that span the latest Cretaceous to early Eocene (70-50 Ma), enabling us to evaluate temporal trends in Os geochemical cycle during this period.

\subsection{Geological setting}

The focus of this study is on the Waipawa and Whangai formations from the East Coast Basin, New Zealand, which extends from East Cape in North Island to the Kaikoura Peninsula in South Island, and about half of which is offshore (Fig. 3.1a). The basin occupied a site on the Pacific subduction margin of Gondwana during the Triassic to early Cretaceous time when rocks of Torlesse Terrane (greywacke) were accreted to form the now weakly metamorphosed and deformed mudstone and sandstone basement (Field et al., 1997; King, 2000). From the mid-Cretaceous, the basin transitioned into a northward-prograding, passive continental margin (Fig. 3.1b) that allowed thick units of Late Cretaceous to Paleogene sediments, eroded from axial ranges, to be deposited onto the continental shelf and slope (King, 2000). The propagation of the modern plate boundary in the early Miocene transformed the setting of the basin from a passive margin to a convergent forearc basin (King, 2000). The additional one sample (Blacks Quarry) from Northland is from an allochthonous sequence, which is inferred to have originated in the North Slope Basin (Fig. 3.1a, b; Hollis et al., 2006). 

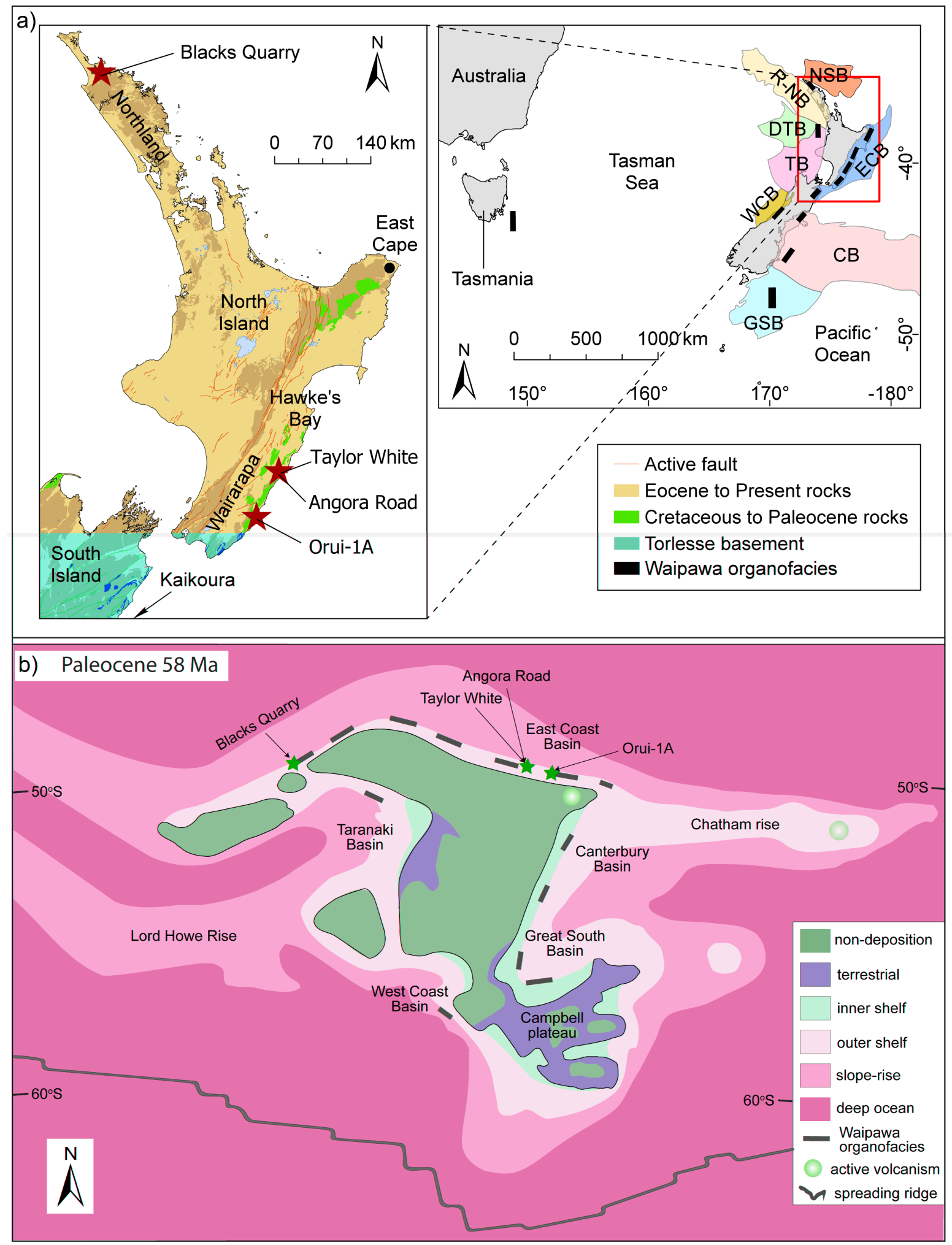

Fig. 3.1. Map of the study area showing sample locations and distribution of Waipawa organofacies in the Southwest Pacific. Geological data extracted from QMAP 1:250K data set (Heron, 2014). Abbreviations: R-NB $=$ Reinga-Northland Basin; NSB $=$ North Slope Basin; DTB $=$ Deepwater Taranaki Basin; $\mathrm{TB}=$ Taranaki Basin; $\mathrm{ECB}=$ East Coast Basin; $\mathrm{WCB}=$ West Coast Basin; $\mathrm{CB}=$ Canterbury Basin; GSB = Great South Basin. b) Paleogeographic reconstruction of the New Zealand region in the late Paleocene (58 Ma; after King, 2000; Hollis et al., 2014). 
The Waipawa Formation is a poorly bedded, brownish-black, organic-rich marine sedimentary unit that ranges in thickness from $\sim 2$ to $80 \mathrm{~m}$ (Moore, 1988; Hollis et al., 2014; Field et al., 2018; Naeher et al., 2019). Foraminiferal paleobathymetry indicates that the formation was deposited in an upper- to mid-slope setting (Field et al., 2018; Naeher et al., 2019). Palynological and palynofacies data suggest that the Waipawa Formation and correlated units were deposited during a sea-level fall (Schiøler et al., 2010; Hollis et al., 2014 and references therein) within a relatively short period of time in the late Paleocene (ca. 0.7 Myr; Fig. 3.2; Hollis et al., 2014). The fall in sea-level is thought to have resulted in increased delivery of terrestrial organic matter to continental slope settings as the exposed nearshore and shelfal areas were largely bypassed (Hines et al., 2019; Naeher et al., 2019). Oscillations in key geochemical parameters (e.g., TOC, hydrogen index $[\mathrm{HI}]$, oxygen index $[\mathrm{OI}], \delta^{13} \mathrm{C}$ and palynofacies) through the formation indicate that influxes of terrestrial organic matter occurred episodically (Naeher et al., 2019). For the most part, the Waipawa Formation conformably overlies the Whangai Formation and the transition into Waipawa organofacies is gradational (Moore, 1989; Naeher et al., 2019). There is, however, an unconformity in some sections where the Waipawa Formation is condensed (Wilson and Moore, 1988; Hollis et al., 2014). The top of the formation is commonly marked by an unconformity (Schiøler et al., 2010; Hollis et al., 2014).

The Whangai Formation is a thick (typically 300-500 m), poorly bedded, variably calcareous and regionally extensive mudstone that consists of the Upper Calcareous, Rakauroa, Te Uri, Porangahau and Kirks Breccia members (Moore, 1988; Field et al., 1997). The Whangai Formation samples analysed in this study are all from the Upper Calcareous Member, which commonly underlies the Waipawa Formation in the East Coast Basin. 


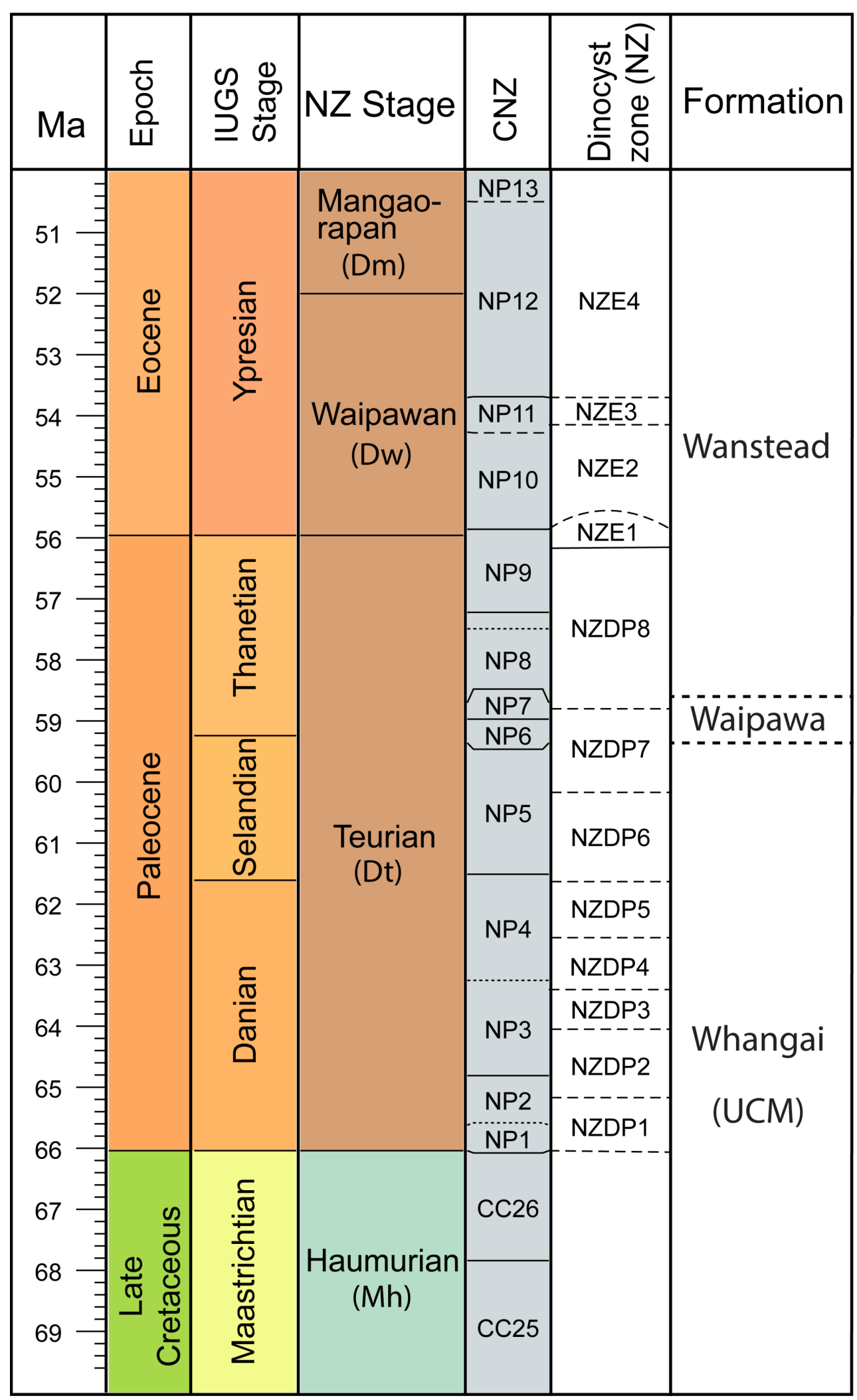

Fig. 3.2. Chronostratigraphic column of the East Coast Basin from the Late Cretaceous to early Eocene (after King, 2000). Nannofossil assemblages are correlated to the biostratigraphic zonation scheme of Martini (1971), with absolute ages for events from Gradstein et al. (2012). Calcareous nannofossils zonation (CNZ) after Crouch et al. (2014). IUGS = International Union of Geological Sciences; UCM $=$ Upper Calcareous Member. 


\subsection{Samples and analytical methodology}

\subsubsection{Samples and sample preparation}

Waipawa Formation samples were collected from the Orui-1A drill core, housed at the New Zealand National Core Store in Featherston, and archived outcrop samples from Taylor White section and Blacks Quarry held at GNS Science. The Orui-1A stratigraphic drill hole was drilled in 2011 to a total depth of 117.3 m near Riversdale, coastal Wairarapa (41 ${ }^{\circ} 3^{\prime} 54.36^{\prime \prime} \mathrm{S}$, 176 ${ }^{\circ} 16.44 "$ E; Fig. 3.1a; Field et al., 2018). Taylor White section is a road-side section exposed on Angora Road (40²7'46.9” S, 176 $28^{\prime} 24.9^{\prime \prime}$ E), near the small settlement of Wimbledon, southern Hawke's Bay (Fig. 3.1a; Bland et al., 2014). The Taylor White section was freshly exposed when road construction was undertaken to widen the Angora Road, allowing for fresh samples to be collected after several meters of rock had been removed (Bland et al., 2014; Naeher et al., 2019). Blacks Quarry is located in the Doubtless Bay, in the Northland region of North Island (345' $40.62^{\prime \prime}$ S, $173^{\circ} 23^{\prime} 54.24^{\prime \prime}$ E, Fig. 3.1a). Whangai Formation samples were also obtained from a collection of archived outcrop samples held at GNS Science, previously collected from the lower part of Angora Road (40 $27^{\prime} 43.2^{\prime \prime} \mathrm{S}$, 176 28'42.2" E; Tayler, 2011; Hollis et al., 2014), termed the 'Angora Road section' in the present study. Stratigraphically, the Whangai Formation samples are located ca. $17-35 \mathrm{~m}$ below the base of Waipawa Formation (Tayler, 2011; Hollis et al., 2014). The Orui-1A samples were obtained from a $3.2 \mathrm{~m}$ stratigraphic interval between the depths of 48.4 and $51.6 \mathrm{~m}$, with sample spacing ranging from 13 to $72 \mathrm{~cm}$ and each sample representing a stratigraphic interval of approximately 3 to $5 \mathrm{~cm}$. Care was taken to avoid zones of faulting, brecciation and calcite veining that were present in the core. In contrast, the Taylor White samples are from a much thicker stratigraphic interval of approximately $50 \mathrm{~m}$ and were not collected specifically for ReOs geochronology which requires that sampling to be done over a small stratigraphic interval to minimize possible variation in initial ${ }^{187} \mathrm{Os} /{ }^{188} \mathrm{Os}$ ratios (Cohen et al., 1999; Selby and Creaser, 2003; Kendall et al., 2009b). The Taylor White samples were included to examine how varying depositional conditions within the Waipawa Formation, as shown by stratigraphic oscillations in key geochemical parameters (Naeher et al., 2019), may have affected the Re-Os systematics. A total of 23 samples are examined in this study; 18 from the Waipawa Formation and 5 from Whangai Formation. Sample processing was undertaken in the rock crushing facility at Victoria University of Wellington (VUW). Rock samples (80-100 g) were polished to remove any surface contamination and drilling marks from the core material following the 
protocols of Kendall et al. (2009a). The samples were then dried in an oven overnight at $40{ }^{\circ} \mathrm{C}$, broken into small pieces without direct metal contact, and powdered using an agate mill.

\subsubsection{Bulk pyrolysis and sulfur analyses}

Bulk pyrolysis and sulfur data obtained by this study were used in conjunction with published geochemical and palynofacies datasets (Naeher et al., 2019) to assess the variation in organic matter type and depositional conditions within the Waipawa and Whangai formations. Bulk pyrolysis analyses for the Orui-1A and Angora Road samples were undertaken at GNS Science following published methods (Naeher et al., 2019). In brief, powdered samples were analysed using a Weatherford Laboratories TOC/TPH Source Rock Analyser (SRA) to obtain key geochemical parameters that include TOC, total volatile (S1) and pyrolysable (S2) hydrocarbons, temperature of maximum pyrolysis yield $\left(\mathrm{T}_{\max }\right), \mathrm{HI}$ and $\mathrm{OI}$. The temperature programme used was $300{ }^{\circ} \mathrm{C}$ isothermal for $3 \mathrm{~min}$, then increased at a rate of $25^{\circ} \mathrm{C} \mathrm{min}-1$ to $650{ }^{\circ} \mathrm{C}$ (isothermal at $650{ }^{\circ} \mathrm{C}$ for $1 \mathrm{~min}$ ) to pyrolyse the kerogen, followed by oxidation at $630{ }^{\circ} \mathrm{C}$ for $20 \mathrm{~min}$. A standard (Institut Français du Pétrole [IFP] standard 160000) was run at the start and finish of each sample sequence, and after every 8 samples to check for data quality. Bulk pyrolysis data for the Taylor White samples were obtained from Naeher et al. (2019). Bulk pyrolysis data for the Blacks Quarry sample were obtained previously from the Geological Survey of Canada, Calgary, using a Rock-Eval 6 instrument.

Analyses of total sulfur content ( $\mathrm{S}_{\text {tot }}$ ) and forms of sulfur for the Orui-1A, Angora Road and Blacks Quarry samples were undertaken by CRL Energy Ltd in Lower Hutt, New Zealand, using standard procedures (Naeher et al., 2019). In brief, Stot was determined by hightemperature $\left(1350{ }^{\circ} \mathrm{C}\right)$ tube furnace combustion, based on the ASTM Standard D4239 technique, using a Leco Truspec Sulfur Analyser model 630-100-700 (Naeher et al., 2019). The different forms of sulfur were determined according to Australian Standard AS1038.112002 where sulfate sulfur $\left(\mathrm{S}_{\text {sul }}\right)$ is extracted by $10 \% \mathrm{HCl}$ and determined gravimetrically after purification and precipitation. The $\mathrm{HCl}$ extracted residue is then decomposed with nitric acid and oxidised using $\mathrm{H}_{2} \mathrm{O}_{2}$ to obtain pyritic iron. The pyritic iron is measured by atomic absorption spectroscopy and used to calculate pyritic sulfur $\left(\mathrm{S}_{\mathrm{pyr}}\right)$. Organic sulfur ( $\left.\mathrm{S}_{\mathrm{org}}\right)$ is obtained by subtracting the sum of $S_{\text {sul }}$ and $S_{\text {pyr }}$ from $S_{\text {tot. }}$ Sulfur data for the Taylor White samples were available from Naeher et al. (2019) and were also analysed by CRL Energy Ltd using the same procedures and instrumentation. 


\subsubsection{Re-Os analyses}

Full Re-Os isotope analyses of the samples were carried out at Durham University's laboratory for source rock and sulfide geochronology and geochemistry following previously published protocols for isolation, purification and measurement by isotope dilution - negative thermal ionization mass spectrometry (e.g., Cumming et al., 2012; Jones et al., 2018). In brief, a known amount of rock powder (300-500 mg) was spiked with a known amount of ${ }^{190} \mathrm{Os}+{ }^{185} \mathrm{Re}$ mixed tracer solution and digested using $\mathrm{CrO}_{3}-\mathrm{H}_{2} \mathrm{SO}_{4}$ solution in a sealed Carius tube at $220{ }^{\circ} \mathrm{C}$ for $48 \mathrm{~h}$. Osmium was separated and purified from the solution using solvent extraction and microdistillation methods. The residual Re bearing solution was evaporated to dryness at $80{ }^{\circ} \mathrm{C}$ and purified by $\mathrm{NaOH}$-acetone solvent extraction and further purified using $\mathrm{HCl}-\mathrm{HNO}_{3}$ anion exchange chromatography.

The resulting Re and Os fractions were loaded onto nickel and platinum wire filaments, respectively, and their isotopic compositions measured on a Thermo Scientific TRITON Negative Thermal Ionisation Mass Spectrometer housed at the Arthur Holmes Laboratory, Durham University. Rhenium isotopic composition was measured via static collection mode in Faraday cups, with Os measured via ion-counting through a secondary electron multiplier (SEM) in a peak-hopping mode. Total procedural blanks during this study were $14.6 \pm 0.16 \mathrm{pg}$ Re and $50 \pm 0.01 \mathrm{fg}$ Os, with an ${ }^{187} \mathrm{Os} /{ }^{188} \mathrm{Os}$ value of $0.22 \pm 0.08(\mathrm{n}=4)$. In-house standards for both Re (Restd; Selby, 2007) and Os (DROsS; Nowell et al., 2008) were run with every batch of samples to monitor instrument reproducibility and ensure data quality. The Re standard solution yields an average ${ }^{185} \mathrm{Re} /{ }^{187} \mathrm{Re}$ ratio of $0.59843 \pm 0.00193(\mathrm{n}=7)$ that is similar to the established natural ${ }^{185} \mathrm{Re} /{ }^{187} \mathrm{Re}$ ratio of $0.59739 \pm 0.00039$ (Gramlich et al., 1973), and was used to correct for mass fractionation in the measured Re data. The average ${ }^{187} \mathrm{Os} /{ }^{188} \mathrm{Os}$ ratio of the in-house Durham Romil Osmium Standard (DROsS) was $0.16089 \pm 0.00056$ ( $\mathrm{n}=$ 7), consistent with values reported in previous studies from the same lab (e.g., Cumming et al., 2012; Liu and Selby, 2018, and references therein), and also in excellent agreement with values reported by other laboratories $(0.16078 \pm 0.00024$, Liu and Pearson, 2014; $0.16091 \pm 0.00015$, van Acken et al., 2019). The Re-Os isotopic data, calculated $2 \sigma$ uncertainties for ${ }^{187} \mathrm{Re} /{ }^{188} \mathrm{Os}$ and ${ }^{187} \mathrm{Os} /{ }^{188} \mathrm{Os}$ and the associated error correlation function (rho; Ludwig, 1980), were regressed using the beta version of the Isochron program (Li et al., 2019) which incorporates the benchmark Isoplot algorithm (Ludwig, 2012) and a new approach that employs the Monte 
Carlo sampling method for error propagation. In the Isoplot program, a Model 1 age assumes that the assigned $2 \sigma$ uncertainties and calculated error correlations are the only reason the datapoints scatter from the regression line whereas a Model 3 age assumes that the scatter about the isochron line may be linked to both the assigned analytical errors and other geological factors that produce variation in the initial ${ }^{187} \mathrm{Os} /{ }^{188} \mathrm{Os}$ values (Ludwig, 2012). The Isoplot program separates the two scenarios based on the probability of how well the data fit the regression line. In contrast, the Monte Carlo method makes no prior assumption about the possible causes of scatter in the geochronological results and propagates uncertainties $(2 \sigma)$ from both analytical measurements and model assumptions in a consistent manner irrespective of the probability of fit (Li et al., 2019).

\subsection{Results and discussion}

\subsubsection{Kerogen type and maturity}

The bulk pyrolysis data for the Orui-1A, Taylor White and Angora Road samples (Table 3.1; Fig. 3.3) are consistent with published results for the Waipawa and Whangai formations in the East Coast Basin (Tayler, 2011; Field et al., 2018; Naeher et al., 2019). The TOC within the Waipawa Formation in the Orui-1A core and Taylor White section is highly variable, generally ranging from $0.4-5 \mathrm{wt} \%$ with a mean of $2.2 \mathrm{wt} \%$ (Fig. 3.3a; Naeher et al., 2019). TOC is very high $(\sim 10 \mathrm{wt} \%)$ in the Blacks Quarry sample from the North Slope Basin (Fig. 3.3a). In contrast, the Whangai Formation at Angora Road exhibits low TOC content (mostly $<1 \mathrm{wt} \%$, Fig. 3.3a).

All samples presented in this study are thermally immature as indicated by $\mathrm{T}_{\max }$ values ranging from $396-428^{\circ} \mathrm{C}$ (Fig. 3.3b; Tissot and Welte, 1984), and thus, the observed kerogen types within the two formations are representative of the initial kerogen types which, based on the modified van Krevelen diagram (Fig. 3.3c), varies from Type II to Type III. The wide variations in $\mathrm{T}_{\max }, \mathrm{HI}$ and $\mathrm{OI}$ values displayed by the Waipawa Formation samples in the complete Taylor White sample set in Figure 3.3b and c are a result of stratigraphic variations in kerogen type, in part resulting from changing redox conditions within the depositional environment rather than variations in maturity or sample weathering (Field et al., 2018; Naeher et al., 2019). 


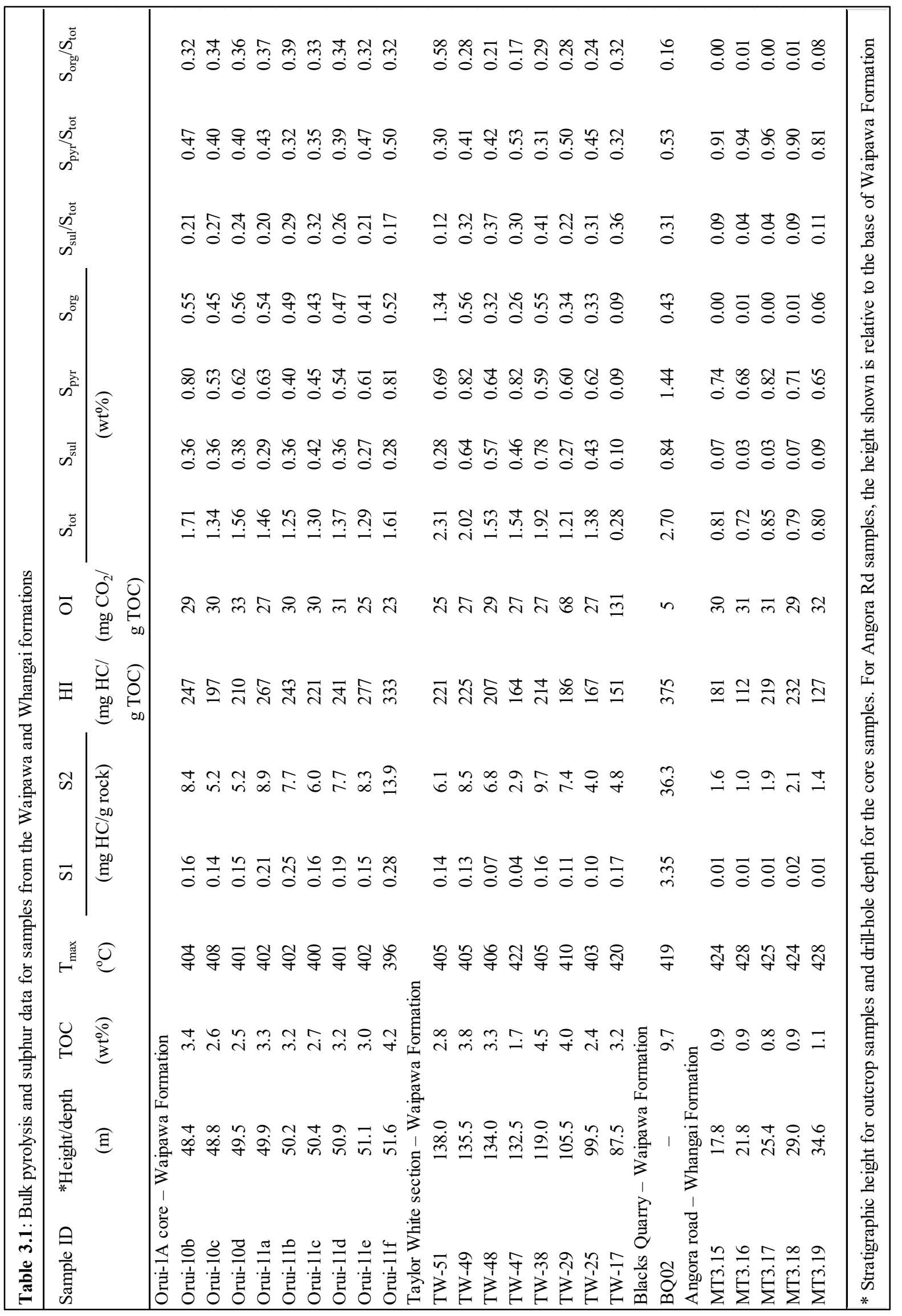


Similar stratigraphic variations in kerogen type also occur within the Waipawa Formation in the Orui-1A core (Field et al., 2018), but are not reflected amongst the Orui-1A samples because these were selected from a thin $(3.2 \mathrm{~m})$ stratigraphic interval with relatively uniform $\mathrm{T}_{\max }, \mathrm{HI}$ and OI values. OI is consistently low ( $\leq 50 \mathrm{mg} \mathrm{CO}_{2} / \mathrm{g}$ TOC) despite high variability in TOC, S2, and HI in the upper part of the Waipawa Formation in both the Taylor White Section and Orui-1A core (Field et al., 2018; Naeher et al., 2019). Palynofacies analyses indicate that the organic matter in the Waipawa Formation is mainly terrestrial woody plant matter whereas marine-sourced amorphous organic matter dominates in the Whangai Formation (Field et al., 2018; Naeher et al., 2019).

a)

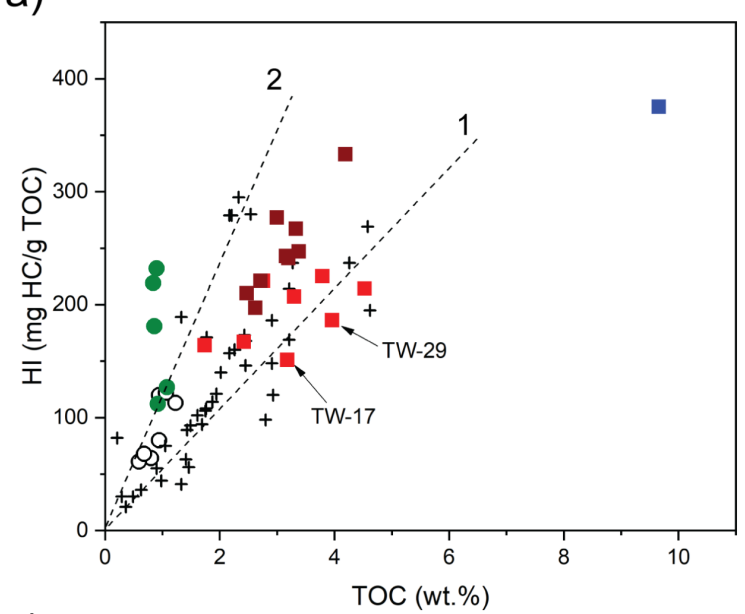

c)

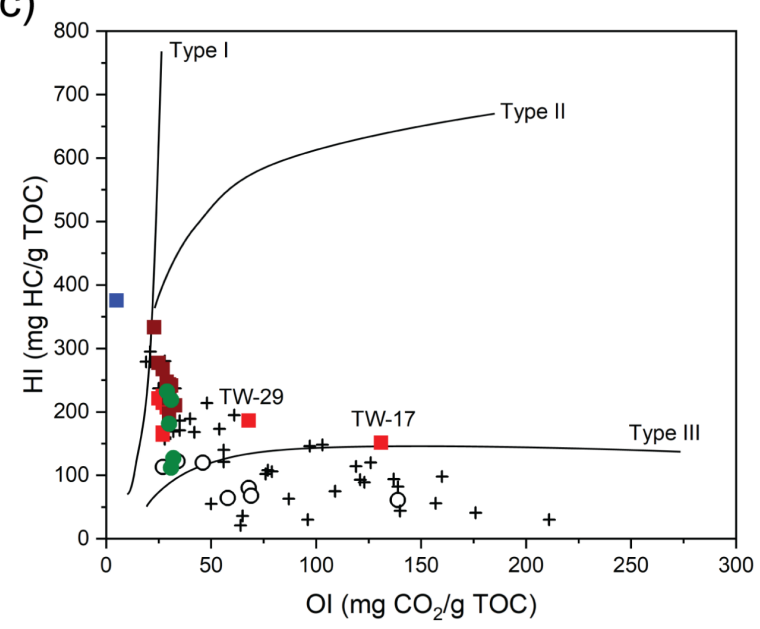

b)

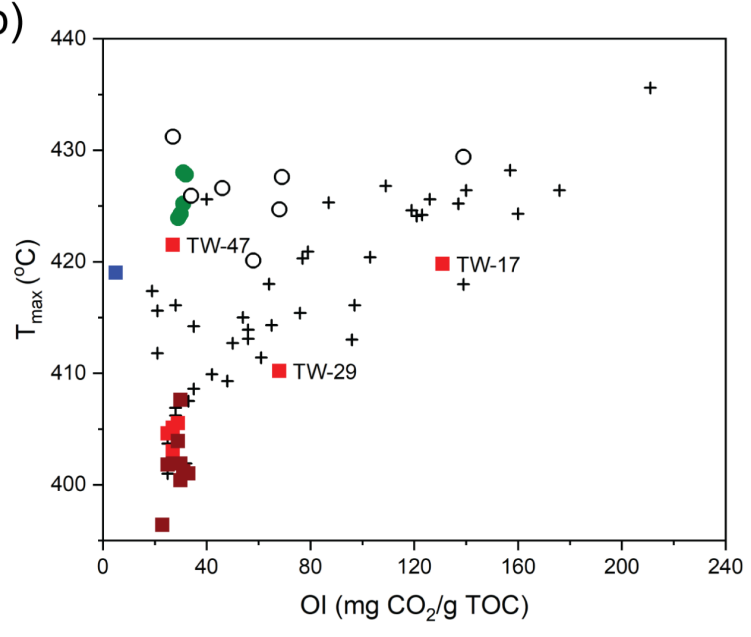

Fig. 3.3. Cross-plots of bulk pyrolysis data identifying the type and maturity of organic matter present in the Waipawa and Whangai formations. a) TOC vs HI, with the oil- (1) and gas-prone (2) trends of Naeher et al. (2019). b) OI vs Tmax. c) Modified van Krevelen diagram showing the general maturation pathways for the main types of organic matter (after Hunt, 1995). 


\subsubsection{Re and Os concentrations}

The Waipawa Formation samples from Orui-1A core, Taylor White section and Blacks Quarry are all enriched in both Re (22.5-85.9 ppb) and Os (397.9-598.6 ppt [ ${ }^{192}$ Os 153.8-254.9 ppt]; Table 3.2) relative to the average concentrations of these elements in the UCC (0.2-2 ppb Re and 30-50 ppt Os; Esser and Turekian, 1993; Sun et al., 2003). The Whangai Formation samples from the Angora Road section are only slightly enriched in Re (3.6-11.8 ppb) and Os (141.8-228.1 ppt [ ${ }^{192}$ Os 55.4-89.0 ppt]) compared to the UCC.

Three Waipawa Formation samples from the Taylor White section are particularly enriched in $\operatorname{Re}(\mathrm{TW}-17=85.9 \mathrm{ppb}, \mathrm{TW}-29=72.9 \mathrm{ppb}$ and TW-51 = 56.3 ppb). The high and variable Re concentrations in the Taylor White samples (Table 3.2) may indicate some form of water mass restriction with varying degrees of replenishment of the water column by Re-rich water from the open ocean (McArthur et al., 2008; van Acken et al., 2019). This is supported by biomarker evidence which suggests that there was persistent water column stratification during the deposition of the formation in the East Coast Basin (Naeher et al., 2019), as well as suggestions of restricted shallow marine depositional setting for Waipawa organofacies in other sedimentary basins such as the Great South and Canterbury basins (Schiøler et al., 2010). Sample TW-17 and TW-29 also show elevated OI values of $131 \mathrm{mg} \mathrm{CO} / \mathrm{g}$ TOC and $68 \mathrm{mg}$ $\mathrm{CO}_{2} / \mathrm{g}$ TOC, respectively, compared to the other Taylor White samples studied here, which are very consistent between 25 and $29 \mathrm{mg} \mathrm{CO}_{2} / \mathrm{g}$ TOC (Table 3.1, Fig. 3.3c). This suggests that these two samples either contain different types of organic matter or they have been affected by oxidative weathering. The latter appears more likely since Re-Os isotope data for the two samples also yield negative initial ${ }^{187} \mathrm{Os} /{ }^{188} \mathrm{Os}\left(\mathrm{Os}_{\mathrm{i}}\right)$ values (Table 3.2), which has been suggested to indicate disturbance of the Re-Os system through oxidative weathering (Jaffe et al., 2002; Georgiev et al., 2012). These two samples are therefore treated as outliers and exclude them from further discussion. 


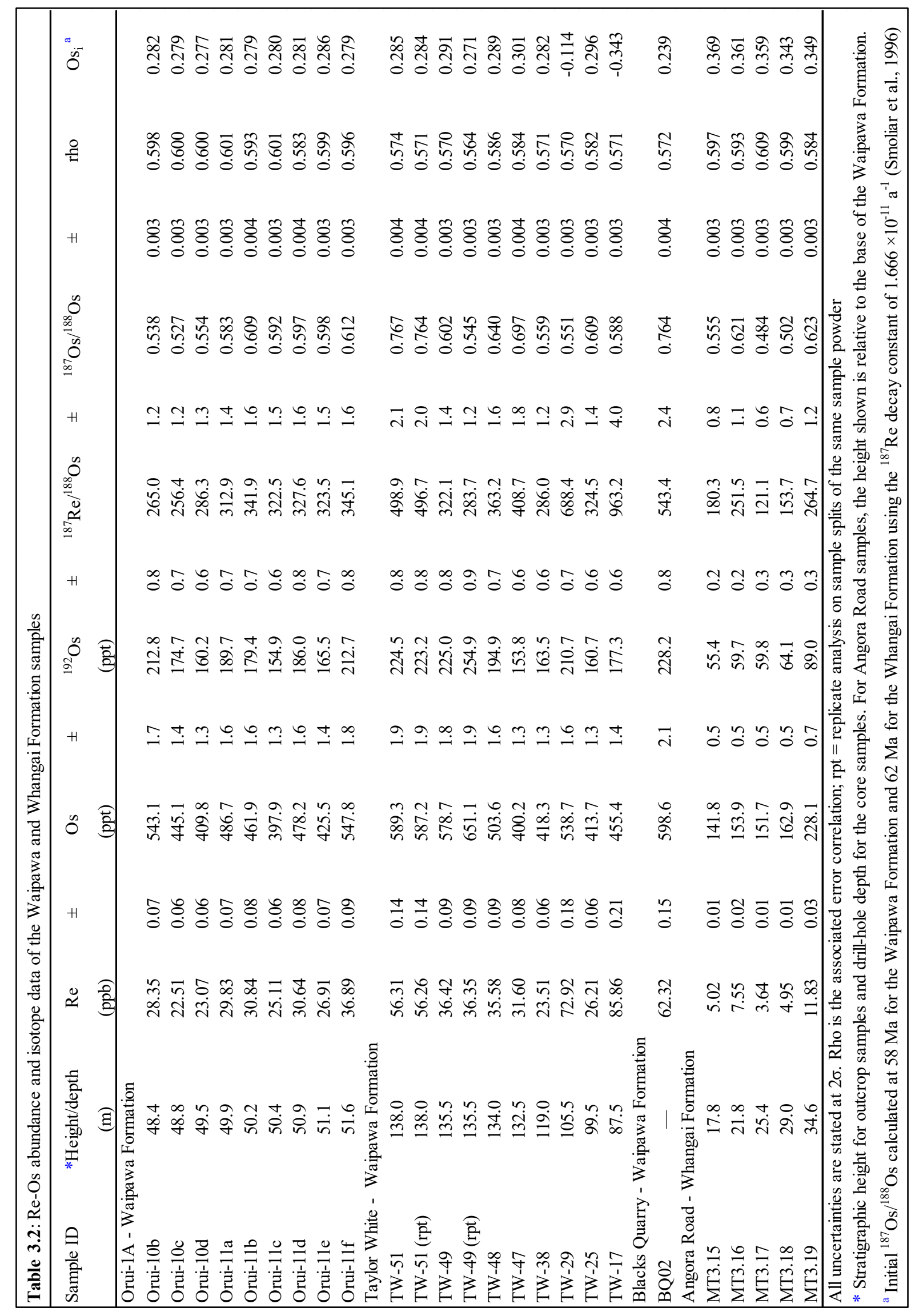




\subsubsection{Re and Os uptake and fractionation in the Waipawa and Whangai formations}

The association of Re and Os with TOC and sulfur may provide useful information on the depositional conditions that enhance uptake of these elements into organic-rich sediments (Cohen et al., 1999; Cumming et al., 2012; Georgiev et al., 2012; Rooney et al., 2012). Strong positive correlations exist between TOC and $\operatorname{Re}\left(\mathrm{R}^{2}=0.91\right)$ and ${ }^{192} \mathrm{Os}\left(\mathrm{R}^{2}=0.73\right)$ in the Orui1A samples (Fig. 3.4a, b), suggesting an uptake mechanism that is linked to the abundance of organic matter (e.g., Georgiev et al., 2012; Rooney et al., 2012 and references therein). The ${ }^{192}$ Os isotope is plotted to avoid the effects of radiogenic in-growth of ${ }^{187} \mathrm{Os}$, allowing for direct comparison of hydrogenous Os concentrations in the different samples. Samples from the Taylor White section show no significant correlation between TOC and $\operatorname{Re}\left(R^{2}=-0.04\right)$ and ${ }^{192} \mathrm{Os}\left(\mathrm{R}^{2}=0.08\right.$; Fig. 3.4a, b), which may indicate possible effects of surficial weathering in these outcrop samples. The apparent correlations between TOC and Re and Os concentrations in the Angora Road samples may be spurious because they rely on one sample that plots away from the other clustered samples (Fig. 3.4a, b). In general, the Waipawa Formation, with higher TOC content, exhibits higher concentrations of Re and Os than the Whangai Formation (Fig. $3.4 a, b)$.
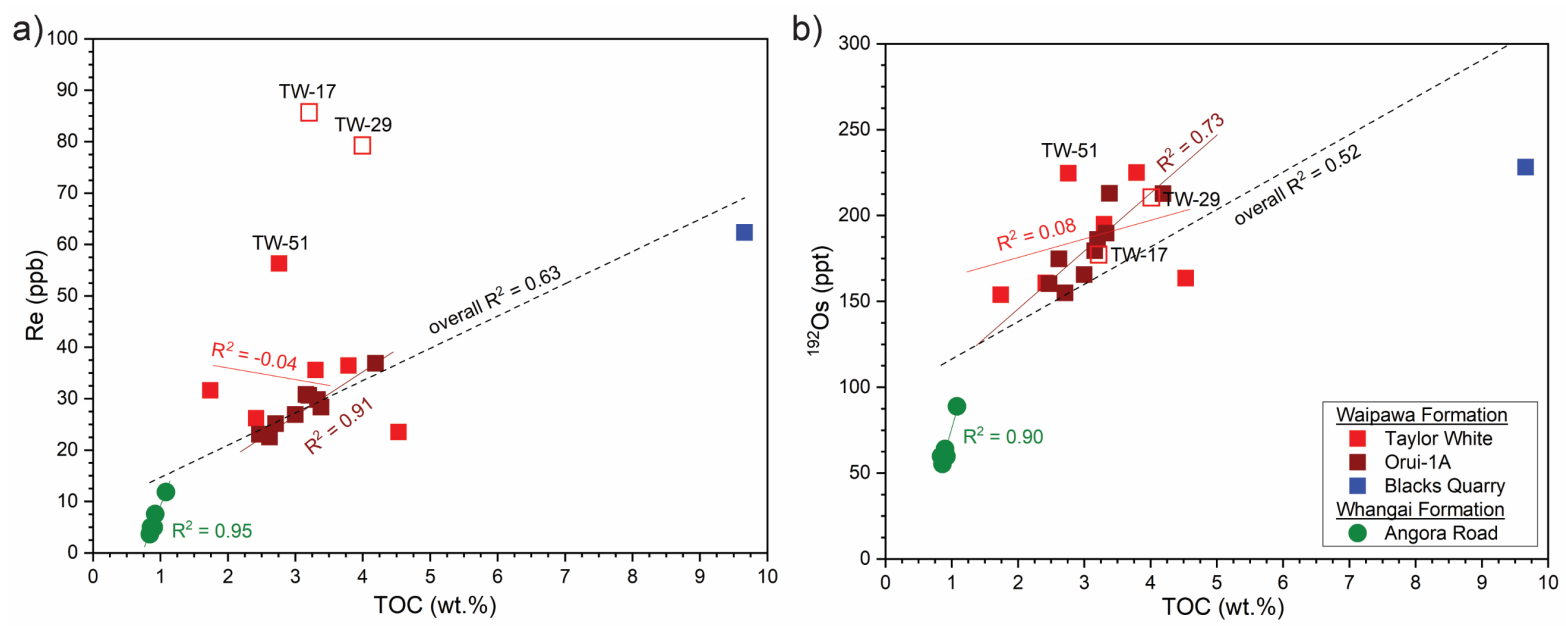

Fig. 3.4. Cross-plots of TOC vs Re (a) and ${ }^{192} \mathrm{Os}$ (b) concentrations in the Waipawa and Whangai formations. Separate trendlines and correlation coefficients are shown by locality. Outlier samples TW17 and TW-29 (unfilled red squares) are excluded from the trend.

The large proportion of terrestrial organic matter (66 to $98 \%$ degraded phytoclasts) in the Waipawa Formation (Schiøler et al., 2010; Hollis et al., 2014; Field et al., 2018; Naeher et al., 2019) does not appear to have impacted the uptake of Re and Os, supporting previous 
interpretations that chelating precursors or fundamental binding sites responsible for uptake of Re and Os are present in all types of organic matter (Cumming et al., 2012; Harris et al., 2013; $\mathrm{Du}$ Vivier et al., 2015). The difference in concentrations of Re and Os in the two formations could also be a factor of the abundance, variability and preservation of organisms such as macroalgae that are components of sedimentary organic matter and which have recently been shown to accumulate Re (up to several hundreds of ppb) and Os (Racionero-Gómez et al., 2016; Rooney et al., 2016; Racionero-Gómez et al., 2017; Ownsworth et al., 2019; Sproson et al., 2020). The accumulation of Re by macroalgae is syn-life and unidirectional i.e. once the living macroalgae absorbs Re, it does not release it back to the water (Racionero-Gómez et al., 2016). However, the study also suggests that Re may be released back to the water column once the macroalgae die and break down, and that depositional conditions which prevent macroalgal degradation (such as anoxia) may be required for much of the accumulated Re to be incorporated into sediments (Racionero-Gómez et al., 2016). The hypoxic conditions during the deposition of the Waipawa Formation, compared to the oxic conditions in the Whangai Formation (Naeher et al., 2019), might therefore be the reason for its higher Re and Os concentrations.

The Waipawa Formation is enriched in $S_{\text {tot }}(1.25-2.31 \mathrm{wt} \%)$ compared to the Whangai Formation (0.72-0.85 wt\%; Fig. 3.5a, b), suggesting deposition under less oxic conditions (Didyk et al., 1978; Georgiev et al., 2012; Naeher et al., 2019). Stot is also positively correlated with the abundance of $\operatorname{Re}\left(\mathrm{R}^{2}=0.84\right)$ and ${ }^{192} \mathrm{Os}\left(\mathrm{R}^{2}=0.72\right)$, as well as the ${ }^{187} \mathrm{Re} /{ }^{188} \mathrm{Os}$ ratio $\left(\mathrm{R}^{2}\right.$ $=0.66$ ) in samples from this study (Fig. 3.5a, b, c). This may suggest that the uptake and fractionation of Re and Os are linked to the redox conditions of the depositional environment (Colodner et al., 1993; Crusius et al., 1996; Cohen et al., 1999; Morford and Emerson, 1999; Crusius and Thomson, 2000; Yamashita et al., 2007; Georgiev et al., 2011). However, no simple correlation can be established between Re and Os concentrations and other indicators of redox conditions such as HI and OI (Table 3.1).

The sulfur speciation data [normalised to $\mathrm{S}_{\text {tot }}$ as there is a high variation in $\mathrm{S}_{\text {tot }}$ within the Waipawa Formation (Naeher et al., 2019)] also show that only organic sulfur ( $\mathrm{S}_{\text {org }} / \mathrm{S}_{\text {tot }}$ ) exhibits strong positive correlations with $\mathrm{Re}\left(\mathrm{R}^{2}=0.75\right.$; Fig. 3.6a) and ${ }^{192} \mathrm{Os}\left(\mathrm{R}^{2}=0.77\right.$; Fig. 3.6b) concentrations compared to pyritic sulfur $\left(\mathrm{S}_{\mathrm{pyr}} / \mathrm{S}_{\mathrm{tot}}\right)$ and sulfate sulfur $\left(\mathrm{S}_{\mathrm{sul}} / \mathrm{S}_{\mathrm{tot}}\right)$, which exhibit negative $\left(\operatorname{Re} \mathrm{R}^{2}=-0.68 ;{ }^{192} \mathrm{Os} \mathrm{R}^{2}=-0.80\right.$; Fig. $\left.3.6 \mathrm{c}, \mathrm{d}\right)$ and weak-positive $\left(\mathrm{Re} \mathrm{R}^{2}=0.25 ;{ }^{192} \mathrm{Os}\right.$ 
$\mathrm{R}^{2}=0.39$; Fig. 3.6e, f) correlations, respectively. This supports previous findings that suggested that Re and Os have a greater affinity for organic chelating sites than sulfides (Cohen et al., 1999; Rooney et al., 2012).
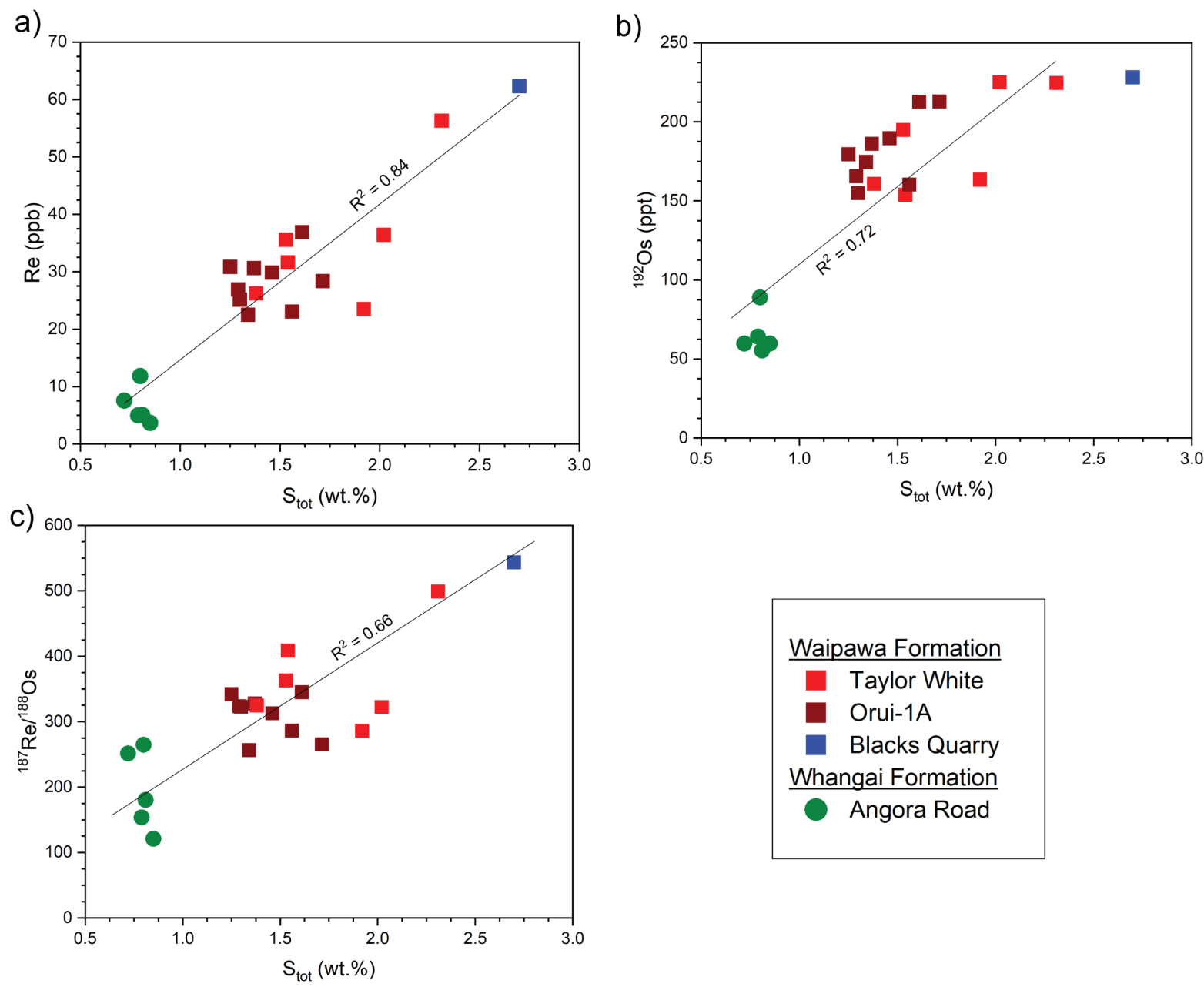

Fig. 3.5. Cross-plots of total sulfur vs a) Re concentrations, b) ${ }^{192}$ Os concentrations and c) ${ }^{187} \mathrm{Re} /{ }^{188} \mathrm{Os}$ for the Waipawa and Whangai formations samples. Outlier samples TW-17 and TW-29 are excluded from these plots. 
a)

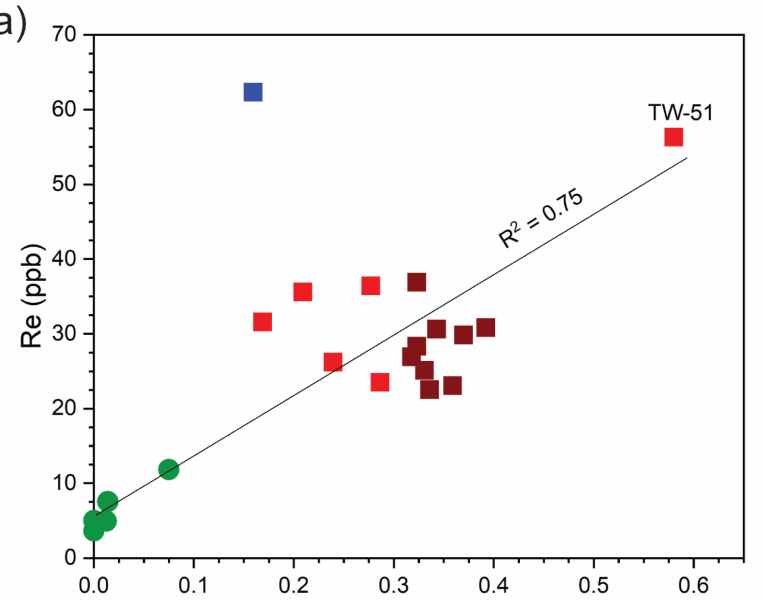

c)

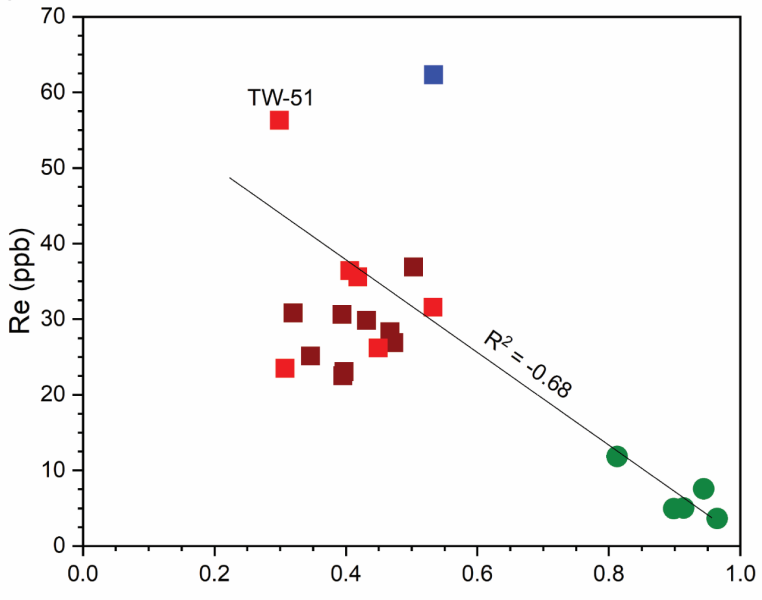

e)

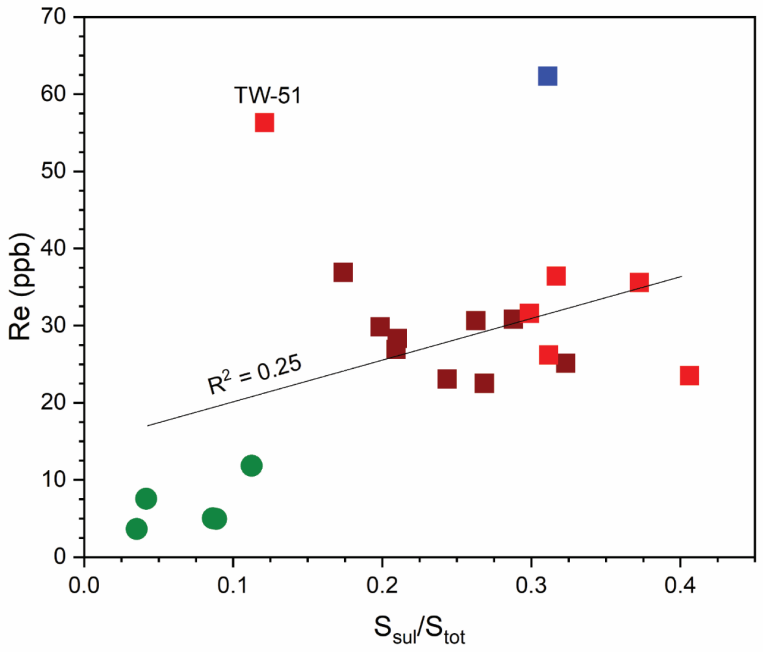

b)

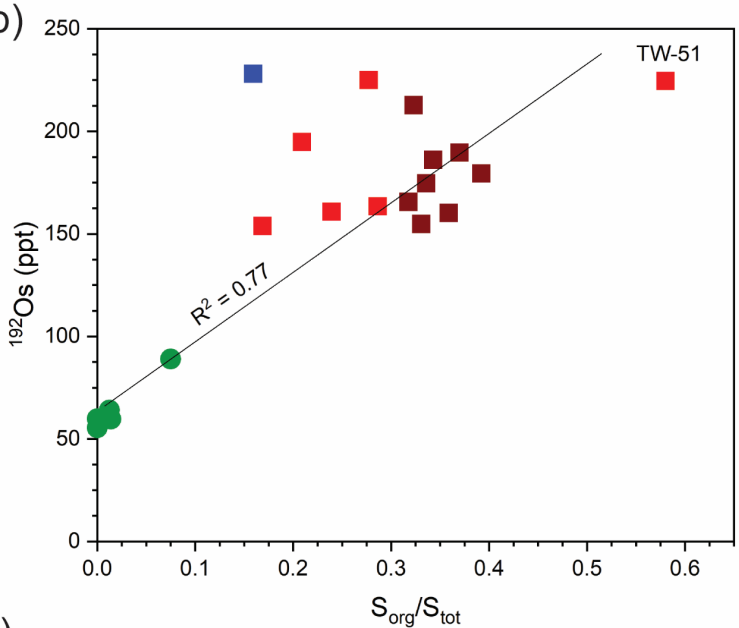

d)
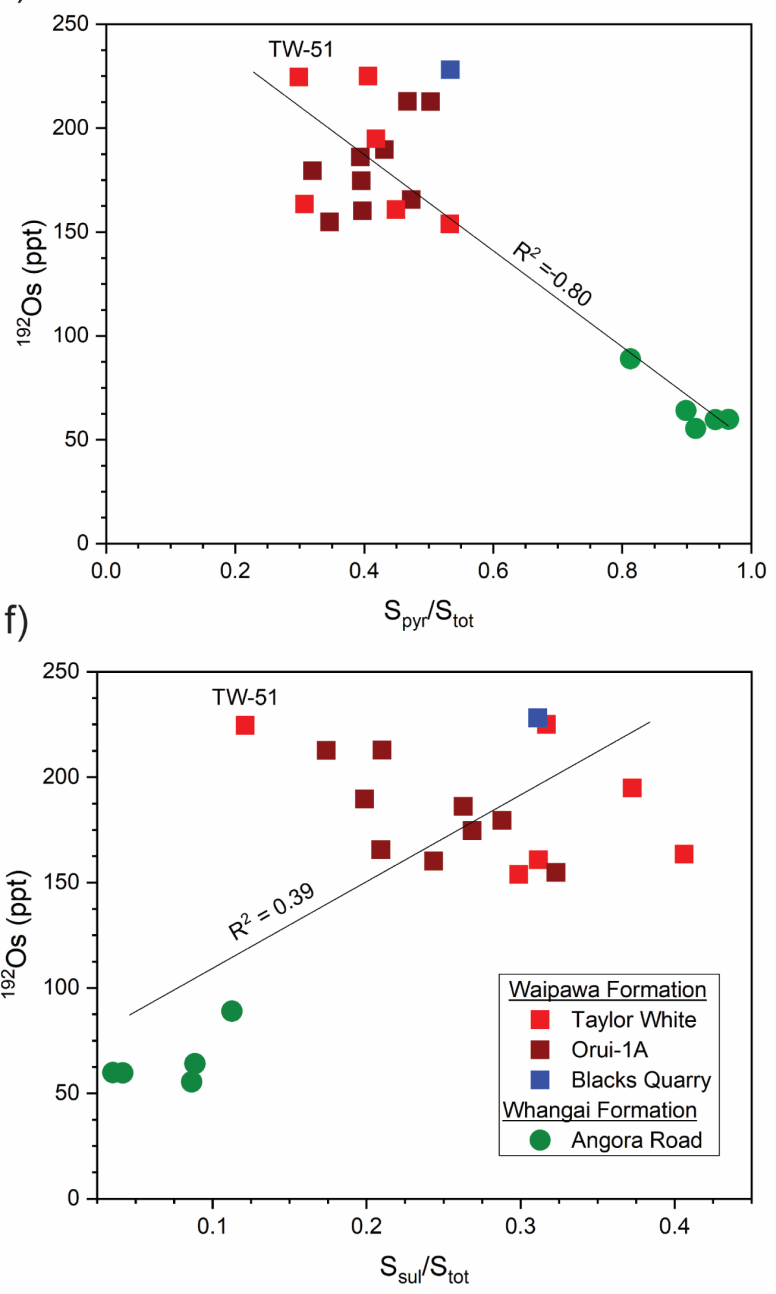

Fig. 3.6. Cross-plots of normalised forms of sulfur and Re and ${ }^{192}$ Os concentrations. Organic sulfur $\left(\mathrm{S}_{\text {org }} / \mathrm{S}_{\text {tot }}\right)$ vs Re (a) and ${ }^{192} \mathrm{Os}(\mathrm{b})$; pyritic sulfur $\left(\mathrm{S}_{\mathrm{pyr}} / \mathrm{S}_{\mathrm{tot}}\right)$ vs Re $(\mathrm{c})$ and ${ }^{192} \mathrm{Os}(\mathrm{d})$; sulfate sulfur $\left(\mathrm{S}_{\mathrm{sul}} / \mathrm{S}_{\mathrm{tot}}\right)$ vs $\operatorname{Re}(\mathrm{e})$ and ${ }^{192} \mathrm{Os}(\mathrm{f})$. Outlier samples TW-17 and TW-29 are excluded from these plots. The legend shown in Figure 3.6f is used for all plots. 
Slow sedimentation, which increases the time of exposure of organic matter to the sedimentwater interface, is another factor that has been considered to play an important role in enhanced uptake of Re and Os in organic-rich sedimentary rocks (Lewan and Maynard, 1982; Selby et al., 2009; Cumming et al., 2012; Rooney et al., 2012). The average sedimentation rate for the Waipawa Formation $(\sim 10.6 \mathrm{~cm} / \mathrm{ky})$ is almost an order of magnitude higher than that of the Whangai Formation ( $\sim 1.1 \mathrm{~cm} / \mathrm{ky}$; Naeher et al., 2019), and also significantly higher than those of several other marine organic-rich rocks previously dated with the Re-Os isotope system $(<2$ cm/ky; e.g., Kendall et al., 2009b; Georgiev et al., 2017; Tripathy et al., 2018). Therefore, if slow sedimentation rate plays a primary control in Re and Os uptake in organic-rich sediments, then the Waipawa Formation would be expected to record low levels of Re and Os. Instead, the average concentrations of $\operatorname{Re}(38.9 \mathrm{ppb})$ and ${ }^{192} \mathrm{Os}(192.6 \mathrm{ppt})$ in the Waipawa Formation are approximately 6 and 3 times higher, respectively, than those of Whangai Formation $(\mathrm{Re}=$ $\left.6.6 \mathrm{ppb} ;{ }^{192} \mathrm{Os}=65.6 \mathrm{ppt}\right)$, indicating that, in this instance, slower sedimentation rates have not had a significant impact on sequestration of Re and Os.

The relatively low levels of Re and Os in the Whangai Formation compared to the Waipawa Formation may simply be a factor of its low TOC content and different depositional environment. However, the $\mathrm{Re}$ and $\mathrm{Os}$ concentrations in the Waipawa Formation are comparable to those reported for other marine shales with similar characteristics, albeit with much lower sedimentation rates. For example, the Upper Jurassic Hekkingen Formation with TOC of 3.08-10.9 wt\%, Type II/III kerogen, anoxic depositional conditions and a sedimentation rate of $1.83 \mathrm{~cm} / \mathrm{ky}$ has average Re and ${ }^{192} \mathrm{Os}$ abundances of $46 \mathrm{ppb}$ and 158.4 ppt, respectively, similar to those of the Waipawa Formation (Langrock and Stein, 2004; Georgiev et al., 2017; Tripathy et al., 2018). Therefore, based on these results, it appears that a slow sedimentation rate does not play a significant role in Re and Os enrichment in organicrich sediments, at least in the range from $1.1 \mathrm{~cm} / \mathrm{ky}$ to $10.6 \mathrm{~cm} / \mathrm{ky}$. This argument is supported by previous studies which suggested that the oxidation effects brought about by a slow sedimentation rate lead to poor preservation of organic matter (Ingall and Cappellen, 1990) and subsequent re-immobilization (precipitation) of Re (Crusius and Thomson, 2000).

\subsubsection{Re-Os geochronology of the Waipawa Formation}

The ${ }^{187} \mathrm{Re} /{ }^{188}$ Os values of samples from the Orui-1A core range from 256.4 to 345.1 and are positively correlated to the ${ }^{187} \mathrm{Os} /{ }^{188} \mathrm{Os}$ values, which range from 0.527 to 0.612 (Fig. 3.7a). 
Regression of the isotope data using the Isoplot algorithm yields a Model 3 isochron age of $58.1 \pm 3.9 \mathrm{Ma}(\mathrm{n}=9$; Mean Square of Weighted Deviates $[\mathrm{MSWD}]=4.1)$, with an Osi of 0.28 \pm 0.02 (Fig. 3.7a). When the Monte Carlo method is used, the age $(58.1 \pm 4.4 \mathrm{Ma})$ and $\mathrm{Osi}_{\mathrm{i}}$ $(0.28 \pm 0.02)$ are the same as those of the Isoplot method, except that the age uncertainty is slightly greater in the Monte Carlo method due to a higher percentage of the uncertainties (61\%) being related to the model age calculation (Li et al., 2019; Fig. 3.7b). Precise Re-Os dating of sedimentary rocks requires that: 1) the initial ${ }^{187} \mathrm{Os} /{ }^{188} \mathrm{Os}$ ratios $\left(\mathrm{Osi}_{\mathrm{i}}\right)$ are identical, 2) there is a sufficient spread in ${ }^{187} \mathrm{Re} /{ }^{188}$ Os ratios of at least a few hundred units, and 3) the ReOs system remains undisturbed (Cohen et al., 1999; Selby and Creaser, 2005a). The obtained Osi values for Orui-1A samples are all nearly identical, ranging from $0.277-0.286$, with a mean of $0.280 \pm 0.002$ (1SD; Table 3.2). Only sample Orui-11e shows a slightly larger deviation from the mean $\mathrm{Osi}_{\mathrm{i}}(0.006)$ and as a result, exhibits the largest standard error of prediction $(0.92 \%)$ from the line of best-fit through the data (Fig. 3.7a). Analytical uncertainties, variation in the composition of Os during deposition and post-depositional processes such as brecciation and calcite veining that were present in the core are some of the possible reasons for the variance in the Osi of sample Orui-11e. Regression of the Re-Os data without this sample (Orui11e) using the Isoplot program yields a more precise Model 1 age of $57.4 \pm 1.7 \mathrm{Ma}\left(\mathrm{Os}_{\mathrm{i}}=0.28\right.$ $\pm 0.01, \mathrm{n}=8, \mathrm{MSWD}=1.5$; Fig. 3.7c). In comparison, the Monte Carlo method yields an age of $57.5 \pm 3.5 \mathrm{Ma}\left(\mathrm{Os}_{\mathrm{i}}=0.28 \pm 0.02\right.$; Fig. $\left.3.7 \mathrm{~d}\right)$, which indicates that the Model 1 scenario of the Isoplot program underestimated the age uncertainties by $\sim 50 \%$. The larger uncertainties in the obtained Re-Os age is suggested to have resulted from the limited spread in both ${ }^{187} \mathrm{Re} /{ }^{188} \mathrm{Os}$ (80 units) and ${ }^{187} \mathrm{Os} /{ }^{188} \mathrm{Os}(0.085$ units) displayed by the Orui-1A samples, in addition to uncertainties from analytical measurements (Selby et al., 2009; Rooney et al., 2017; Li et al., 2019). Small ranges in Re-Os ratios have also been reported for other marine and lacustrine organic-rich rocks (Turgeon et al., 2007; Selby et al., 2009; Finlay et al., 2010; Cumming et al., 2012; Zhu et al., 2013; Tripathy et al., 2018) where a lack of variability in organic matter type coupled with a relatively homogenous depositional environment (Cumming et al., 2012) and redox conditions that drawdown Re relative to Os (Turgeon et al., 2007) were suggested as the main reasons for the low spread in Re-Os ratios. A lack of variability in organic matter type is a feature of the Waipawa Formation, especially in the Orui-1A samples, possibly explaining the observed similarity in the Re-Os ratios of these samples. 

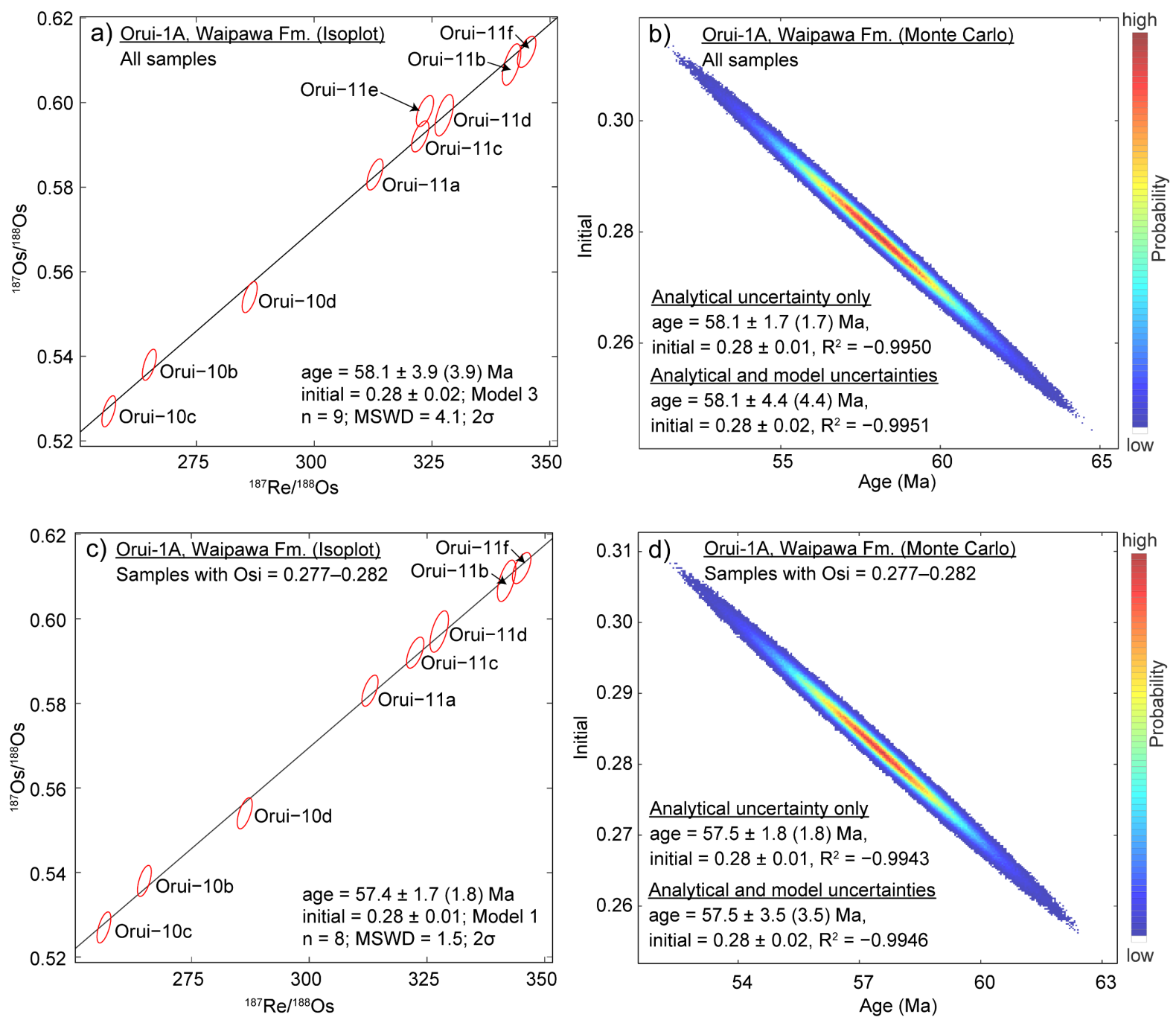

Fig. 3.7. Re-Os geochronological results for the Waipawa Formation samples from Orui-1A core. All samples are plotted in (a) and (b) whereas sample Orui-11e is excluded in (c) and (d). Regression of the Re-Os isotope data together with the $2 \sigma$ uncertainties in the isotope ratios and the associated error correlation functions (rho) were done using the beta version of Isochron program (Li et al., 2019), which incorporates the Isoplot algorithm (a and c; Ludwig, 2012) and the Monte Carlo method (b and d). The Re-Os dates and $\mathrm{Os}_{\mathrm{i}}$ are very similar in both methods, except that the uncertainties from the Monte Carlo method are higher, especially for the Model 1 scenario of the Isoplot program (c; Li et al., 2019). See text for discussion. The bracketed age uncertainty includes the uncertainty in the ${ }^{187} \operatorname{Re}$ decay constant $(\lambda)$, where $\lambda=1.666 \pm 0.0031 \times 10^{-11} \mathrm{yr}^{-1}$ (Smoliar et al., 1996; Selby et al., 2007b).

The ${ }^{187} \mathrm{Re} /{ }^{188}$ Os ratios of samples from the Taylor White section range from 283.7 to 498.9 (excluding the two outliers discussed in Section 3.4.2) and are positively correlated with their corresponding ${ }^{187} \mathrm{Os} /{ }^{188} \mathrm{Os}$ values, which range from 0.551 to 0.767 (Fig. 3.8a). Regression of the isotope data yields a Re-Os date of $58.3 \pm 7.7 \mathrm{Ma}\left(\mathrm{Os}_{\mathrm{i}}=0.29 \pm 0.05 ; \mathrm{MSWD}=28.8\right)$ using the Isoplot algorithm and $58.3 \pm 6.5 \mathrm{Ma}\left(\mathrm{Os}_{\mathrm{i}}=0.29 \pm 0.04 ; \mathrm{n}=6\right)$ using the Monte Carlo method (Fig. 3.8a, b). These Re-Os dates agree well with those derived from the Orui-1A samples, but with larger uncertainties. The high MSWD value associated with the Re-Os date 
from the Isoplot program, relative to the ideal value of $\sim 1$, indicates that the final age uncertainty is controlled by both analytical and geological uncertainties (Ludwig, 2012). This might be expected given the large stratigraphic interval that the samples represent. However, the whole $\sim 80 \mathrm{~m}$ of Waipawa Formation in the Taylor White section is estimated to have been deposited within a relatively short period of time ( 700 ky; Hollis et al., 2014; Naeher et al., 2019 ) and thus, temporal variation in Osi is deemed unlikely to fully explain the uncertainty in the obtained Re-Os date. An alternative explanation is that the episodic influxes of large amounts of terrestrial material during deposition of the formation in the Taylor White section (Naeher et al., 2019) may have supplied high loads of continental-derived Os that introduced localised variation in seawater Osi. Further, the oxidative weathering that affected the Re-Os systematics of the two outlier samples discussed above (Section 4.2) may have also caused subtle effects in the other Taylor White samples.

\subsubsection{Comparison of the Waipawa Formation Re-Os and biostratigraphic ages}

Dating the Waipawa Formation through biostratigraphy has been challenging mainly due to poor preservation and scarcity of age-diagnostic fossils. For example, the boundaries of dinoflagellate (NZDP) or calcareous nannofossil (NP) zones were not precisely located in the Orui-1A core due to the scarcity of fossils (Field et al., 2018). The interval of the Orui-1A core sampled in the present study (48.4 to $51.6 \mathrm{~m}$ ) is also barren of nannofossils and thus, lacks precise age constraints. However, the base of the overlying Wanstead Formation, at $36.84 \mathrm{~m}$, is assigned to Zones NZDP8 and NP8 while the top of Zone NZDP7 is placed at $75.88 \mathrm{~m}$ (Fig. 3.2), which indicates that the Orui-1A samples studied here are of earliest late Paleocene age ( $59 \mathrm{Ma})$. Dinocyst assemblages from several sections in eastern New Zealand indicate that the Waipawa Formation can be correlated with an interval that extends from upper Zone NZDP7 to lower NZDP8 (Crouch et al., 2014; Hollis et al., 2014). Nannofossil biostratigraphy for the Angora Road section also indicates that the Waipawa Formation is correlated with an interval that spans lowermost Zone NP6 to upper Zone NP7 (Fig. 3.2; Hollis et al., 2014). This biostratigraphy was combined with age control from other sections to infer that the Waipawa Formation was deposited over a short period of time ( $\sim 700 \mathrm{ky})$ during the late middle to early late Paleocene (59.4 to $58.7 \mathrm{Ma}$; Hollis et al., 2014). The Re-Os depositional age for the Waipawa Formation obtained from the Orui-1A samples $(57.5 \pm 3.5 \mathrm{Ma})$ overlaps with this 
biostratigraphic age, effectively providing the first direct radiometric age constraint for this formation.
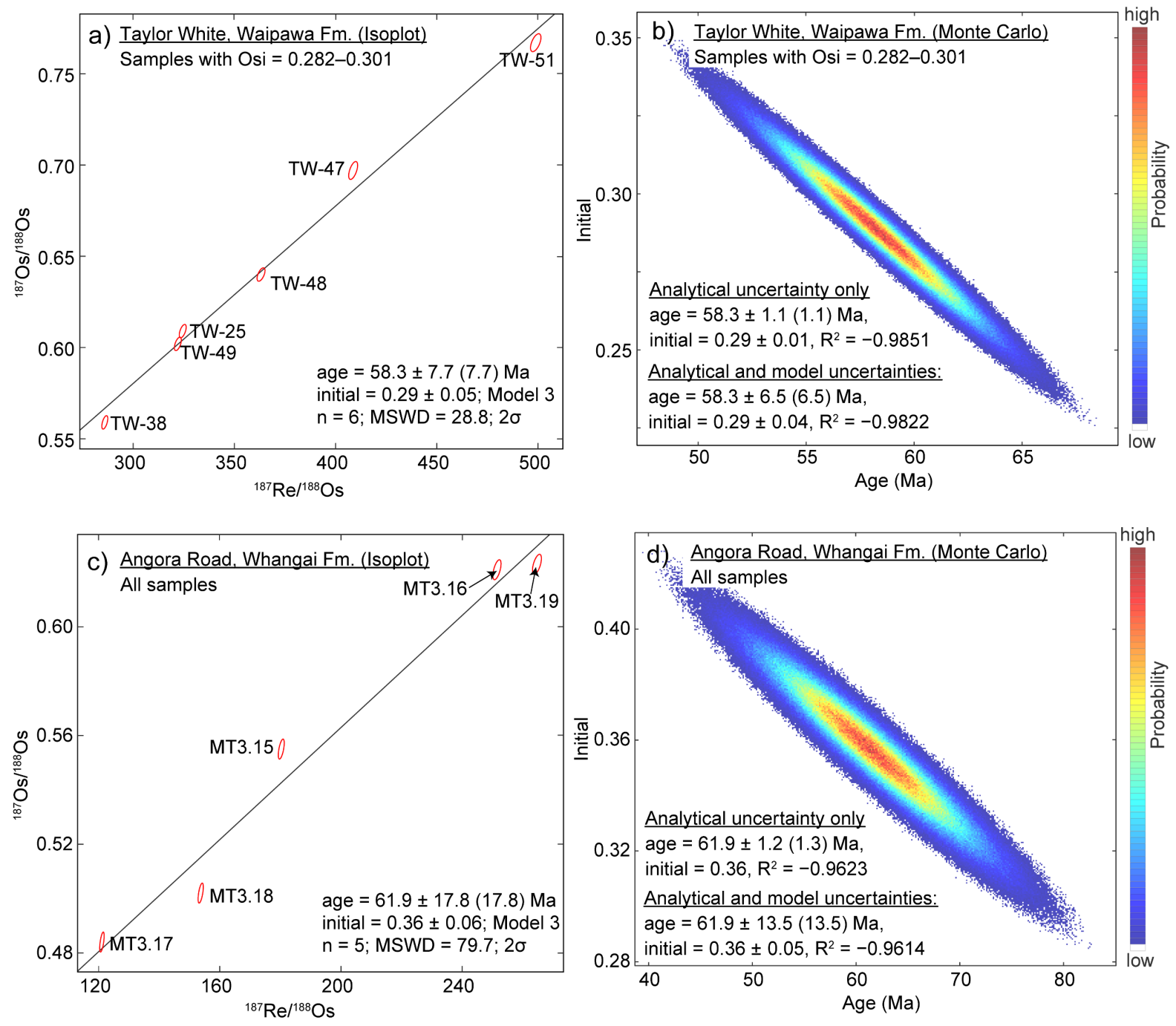

Fig. 3.8. Re-Os geochronological results for the Waipawa Formation samples from the Taylor White section ( $a$ and $b$ ) and Whangai Formation samples from the Angora Road section (c and d). The data were regressed using the beta version of the Isochron program (Li et al., 2019) as described in Fig. 3.7 and in the text. Outlier samples TW-17 and TW-29 are not included in the regression. Note that the uncertainties from the Monte Carlo method are lower than those of the Model 3 scenario of the Isoplot method. See text for discussion. The bracketed age uncertainty includes the uncertainty in the ${ }^{187} \mathrm{Re}$ decay constant $(\lambda)$, where $\lambda=1.666 \pm 0.0031 \times 10^{-11} \mathrm{yr}^{-1}$ (Smoliar et al., 1996; Selby et al., 2007b).

\subsubsection{Re-Os depositional age of the Whangai Formation}

The ${ }^{187} \mathrm{Re} /{ }^{188}$ Os ratios in the Whangai Formation samples from the Angora Road section range from 121.1 to 264.7 and correlate positively with the ${ }^{187} \mathrm{Os} /{ }^{188} \mathrm{Os}$ ratios, which range from 0.484 to 0.633 (Fig. 3.8c). Regression of the isotope data yields imprecise Re-Os date of $61.9 \pm 17.8$ 
$\mathrm{Ma}\left(\mathrm{Osi}_{\mathrm{i}}=0.36 \pm 0.06 ; \mathrm{n}=5 ; \mathrm{MSWD}=79.7\right)$ using the Isoplot algorithm (Fig. 3.8c) and 61.9 $\pm 13.5 \mathrm{Ma}\left(\mathrm{Os}_{\mathrm{i}}=0.36 \pm 0.05\right)$ using the Monte Carlo method (Fig. 3.8d). The large uncertainties in the Re-Os date for these samples reflect the likely variations in Osi over the relatively large time period ( $\sim 2 \mathrm{Ma}$ ) represented by the $\sim 17 \mathrm{~m}$ stratigraphic thickness covered by the Angora Road samples (Kendall et al., 2009b). Over this interval, Osi varies from 0.343-0.369 and tends to become lower up-section, consistent with an overall decreasing trend in Osi from the lowest Whangai Formation sample (0.369) to the highest Waipawa Formation sample in the Taylor White section (0.285). Despite the Re-Os date for the Angora Road samples being imprecise, the mean value of 61.9 Ma is in good agreement with the published age model for the section (Hollis et al., 2014), which combines both calcareous nannofossil and dinoflagellate biostratigraphy (Crouch et al., 2014; Kulhanek et al., 2015), to infer an age range of $\sim 62$ to 61 Ma for the sampled interval.

\subsubsection{Middle-late Paleocene seawater Os isotope composition}

The Osi ratio derived from regression of Re-Os data is interpreted to record the Os isotope composition of the seawater at the time of deposition (Ravizza and Turekian, 1989; Cohen et al., 1999; Selby and Creaser, 2003; Selby et al., 2009). The isochron-derived Osi values for the Waipawa Formation in Orui-1A $(0.28 \pm 0.02)$ and Taylor White $(0.29 \pm 0.04)$ samples, and the Whangai Formation in Angora Road samples $(0.36 \pm 0.05)$ are non-radiogenic, and significantly lower than the present-day seawater ${ }^{187} \mathrm{Os} /{ }^{188} \mathrm{Os}$ value of $\sim 1.02$ to 1.06 (Woodhouse et al., 1999; Peucker-Ehrenbrink and Ravizza, 2000; Gannoun and Burton, 2014). The Osi value (calculated at $58 \mathrm{Ma}$ ) for the one additional Waipawa Formation sample from Blacks Quarry in North Slope Basin $(\sim 0.24 \pm 0.01$; Table 3.2$)$ is also non-radiogenic but lower than those of the Waipawa Formation samples from the Orui-1A and Taylor White sections in the East Coast Basin ( 0.28-0.29). If this sample represents a closed Re-Os system that has not been affected by post-depositional processes, then it would suggest that the Waipawa Formation in the North Slope Basin is either slightly younger or was deposited in a more open marine setting with less radiogenic Osi. The latter appears more likely because the sample is from an allochthonous sequence that was emplaced from an everted marine basin with oceanic volcanic basement, at least $350 \mathrm{~km}$ north of its present location (Ballance and Spörli, 1979; Hollis et al., 2006). The Osi values for the Waipawa Formation samples from the East Coast Basin are broadly similar to those of time correlative units from the central Pacific Ocean 
(0.26-0.33; Fig. 3.9a), suggesting that the basin was mixing with, at least, the wider Pacific Ocean. In addition, the observed Osi values may be indicative of the global seawater ${ }^{187} \mathrm{Os} /{ }^{188} \mathrm{Os}$ values at the time given that paleo-ocean circulation patterns show that the Pacific, Atlantic and Indian oceans were all connected 59 Ma ago (Fig. 3.9a; Haq, 1981; Barron and Peterson, 1991; Thomas et al., 2003; Batenburg et al., 2018).

A composite record of ${ }^{187} \mathrm{Os} /{ }^{188} \mathrm{Os}$ values from sedimentary rocks spanning the period between 70 and $50 \mathrm{Ma}$ and including the ${ }^{187} \mathrm{Os} /{ }^{188} \mathrm{Os}$ values for the Waipawa and Whangai formations in the present study (Fig. 3.9b), provides a broader temporal context in which the source of the non-radiogenic Os in the Paleocene can be examined. The ${ }^{187} \mathrm{Os} /{ }^{188} \mathrm{Os}$ value for the Waipawa Formation is based on the mean Osi values [calculated at $59 \mathrm{Ma}$ - the established biostratigraphic age (Hollis et al., 2014); Table 3.2] for the Orui-1A samples which, unlike the Taylor White samples, demonstrate an isochronous Re-Os relationship (Fig. 3.7). The obtained composite record highlights a progressive shift to low ${ }^{187} \mathrm{Os} /{ }^{188} \mathrm{Os}$ ratios from a value of $\sim 0.61$ in the latest Cretaceous ( $\sim 68 \mathrm{Ma}$ ) to $\sim 0.28$ in late Paleocene (Fig. 3.9b). In particular, the ${ }^{187} \mathrm{Os} /{ }^{188} \mathrm{Os}$ value of $\sim 0.28$ at ca. $59 \mathrm{Ma}$ during the deposition of the Waipawa Formation coincides with the minimum before the marine ${ }^{187} \mathrm{Os} /{ }^{188} \mathrm{Os}$ trend increases leading up to the Paleocene-Eocene boundary. Superimposed upon the overall decreasing trend in ${ }^{187} \mathrm{Os} /{ }^{188} \mathrm{Os}$ values from the latest Cretaceous to the late Paleocene is the abrupt negative excursion at ca. $66 \mathrm{Ma}$ associated with the Cretaceous-Paleogene (K-Pg) impact event (Fig. 3.9b; Pegram and Turekian, 1999; Ravizza and VonderHaar, 2012). Extra-terrestrial impact events are distinguished in the marine Os record by abrupt shifts to low ${ }^{187} \mathrm{Os} /{ }^{188}$ Os values (resulting from a sudden influx of non-radiogenic Os), followed by recovery to pre-impact values over a short duration of time (Paquay et al., 2008; Ravizza and VonderHaar, 2012). In contrast, the gradual nature of the long-term decline in ${ }^{187} \mathrm{Os} /{ }^{188} \mathrm{Os}$ values from the latest Cretaceous to late Paleocene indicates either a progressive increase in the supply of non-radiogenic Os or diminished supply of radiogenic Os from continental weathering. 


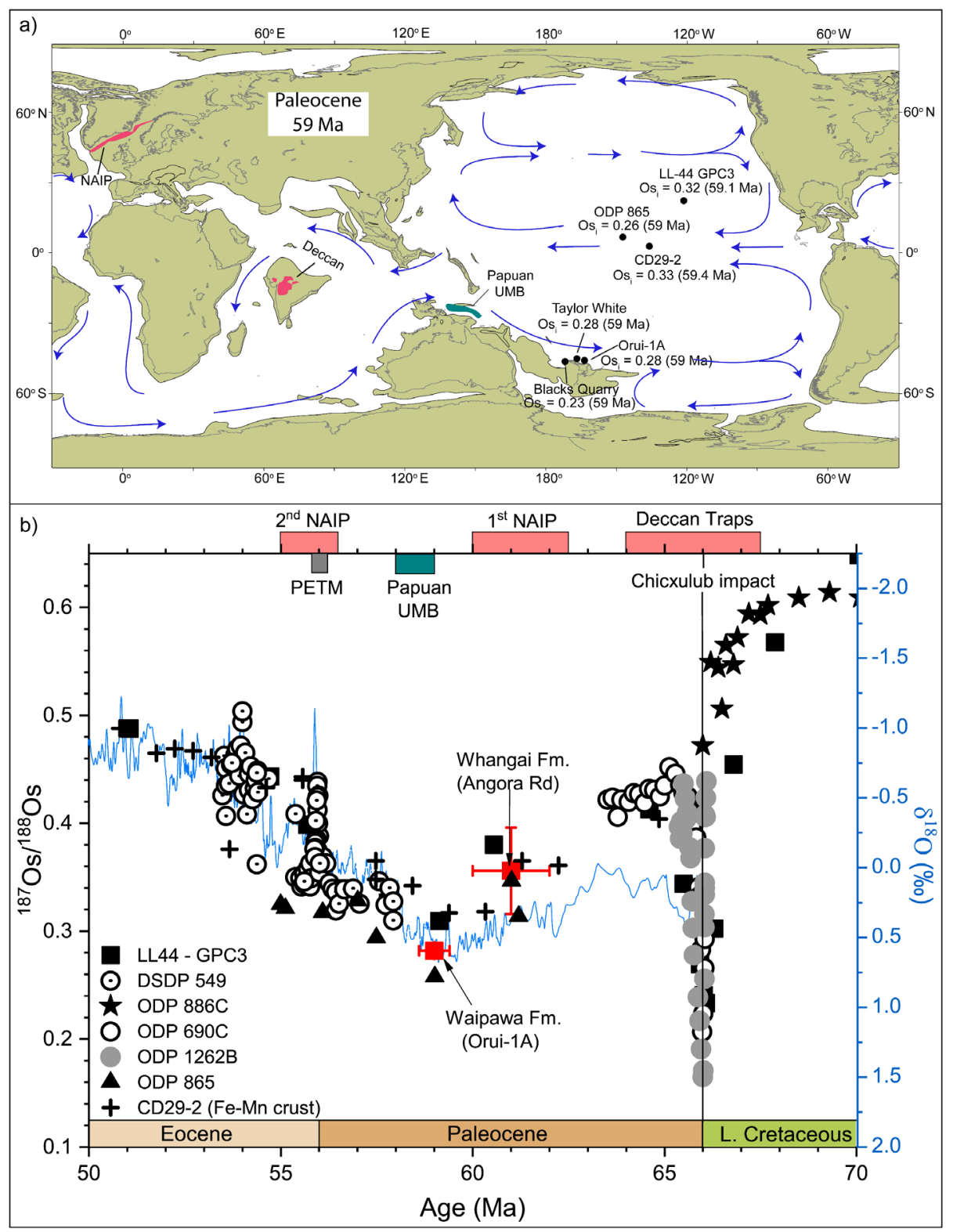

Fig. 3.9. a) A GPlates global paleogeographic reconstruction of the late Paleocene (59 Ma) using the relative finite rotations, continental polygons and coastlines from Matthews et al. (2016), placed in the global paleomagnetic reference frame of Torsvik et al. (2012). Ocean circulation patterns are inferred from Haq (1981), Barron and Peterson (1991), Thomas et al. (2003) and Batenburg et al. (2018). b) composite ${ }^{187} \mathrm{Os} /{ }^{188} \mathrm{Os}$ record of the latest Cretaceous to early Eocene period $(70-50 \mathrm{Ma})$. The ${ }^{187} \mathrm{Os} /{ }^{188} \mathrm{Os}$ value for the Waipawa Formation is based on mean Osi ratios (calculated at $59 \mathrm{Ma}$ ) from Orui-1A samples (see text for discussion) whereas that of the Whangai Formation is derived from the Angora Road samples isochron. The benthic foraminiferal oxygen isotope $(\delta 18 \mathrm{O})$ record from ODP site 1209 is shown for the period between 66 and $50 \mathrm{Ma}$ (Westerhold et al., 2017; 2018). Os isotope data sources: LL44-GPC3 = Pegram and Turekian (1999); DSDP 549 = Ravizza et al. (2001); CD29-2 $=$ Klemm et al. (2005); ODP 886C = Ravizza (2007); ODP 690C and ODP 1262B = Ravizza and VonderHaar (2012) and ODP $865=$ Rolewicz (2013). Not shown are initial ${ }^{187} \mathrm{Os} /{ }^{188} \mathrm{Os}$ ratios for late Maastrichtian (65.5 to 68.5 Ma) sediments from ODP 690, DSDP 577 and DSDP 525 (Robinson et al., 2009) that agree well with the data from Ravizza (2007) and Ravizza and VonderHaar (2012). NAIP = North Atlantic Igneous Province; Papuan UMB = Papuan Ultramafic Belt; PETM = Paleocene-Eocene Thermal Maximum.A number of events have been proposed as potential sources of the non- 
radiogenic Os during this period. These include emplacement of large igneous provinces such as the Deccan Traps (Ravizza and Peucker-Ehrenbrink, 2003a; Robinson et al., 2009) and the first eruptive phase of the North Atlantic Igneous Province (Peucker-Ehrenbrink and Ravizza, 2012), as well as weathering of exhumed large ophiolites such as the Papuan Ultramafic Belt (Fig. 3.9b; Lus et al., 2004; Peucker-Ehrenbrink and Ravizza, 2012). It is unclear, however, how much these events contributed to the ${ }^{187} \mathrm{Os} /{ }^{188} \mathrm{Os}$ decline given the significant time gaps between them and the long duration of the decline. Interestingly, though, the ${ }^{187} \mathrm{Os} /{ }^{188} \mathrm{Os}$ record is correlated with global temperature proxies $\left(\delta^{18} \mathrm{O}\right.$, TEX 86$)$ and the carbon isotope $\left(\delta^{13} \mathrm{C}\right)$ record for much of the Paleocene and into the early Eocene (Figs 3.9b, 3.10). The temperature records indicate moderate cooling between 64 and 58 Ma followed by a more pronounced warming trend (by $\sim 10^{\circ} \mathrm{C}$ ) to around $53 \mathrm{Ma}$ (Bijl et al., 2009; Hollis et al., 2012; Hollis et al., 2014; Westerhold et al., 2018). The temperature minimum at around 58.7 Ma coincides with the ${ }^{187} \mathrm{Os} /{ }^{188}$ Os minimum and deposition of the Waipawa Formation (Fig. 3.10b, c).

The simplest interpretation for these observations is that the gradual shift to low ${ }^{187} \mathrm{Os} /{ }^{188} \mathrm{Os}$ values in the middle-late Paleocene relates to a reduction in global rates of continental weathering as temperature (and thus, the hydrological cycle) decreased, whereas the positive shift from the late Paleocene to early Eocene relates to increased rates of continental weathering in response to rising temperatures and an accelerated hydrological cycle (Zachos et al., 2008). This interpretation, although based on an assumption that fluxes of non-radiogenic Os to the ocean remain relatively constant during this period, is supported by previous findings that attributed the positive excursion in the ${ }^{187} \mathrm{Os} /{ }^{188} \mathrm{Os}$ ratios at the Paleocene-Eocene Thermal Maximum (PETM) to increased chemical weathering from an accelerated hydrological cycle, albeit on a much shorter time scale and a more severe climatic perturbation (Ravizza et al., 2001; Wieczorek et al., 2013; Dickson et al., 2015).

The $\delta^{13} \mathrm{C}$ record follows a similar trend to those of the $\delta^{18} \mathrm{O}$ and TEX 86 records, but with a maximum at around $58 \mathrm{Ma}, \sim 1$ my later than the temperature and ${ }^{187} \mathrm{Os} /{ }^{188} \mathrm{Os}$ minimum (Fig. 3.10a). Temporal variations in the stable carbon isotope composition have been attributed to many factors, the discussion of which is beyond the scope of this study. However, the broad correlation of ${ }^{187} \mathrm{Os} /{ }^{188} \mathrm{Os}$ with $\delta^{13} \mathrm{C}$ is consistent with the inference that continental weathering patterns may have contributed to the observed middle Paleocene to early Eocene ${ }^{187} \mathrm{Os} /{ }^{188} \mathrm{Os}$ record. This is because enhanced weathering of isotopically evolved organic-rich rocks would 
have driven the seawater ${ }^{187} \mathrm{Os} /{ }^{188} \mathrm{Os}$ ratios to more radiogenic values and shifted the carbon isotope composition to lighter values (Ravizza and Esser, 1993). This explanation, however, requires that there was exposure of large amounts of significantly older organic-rich rocks.

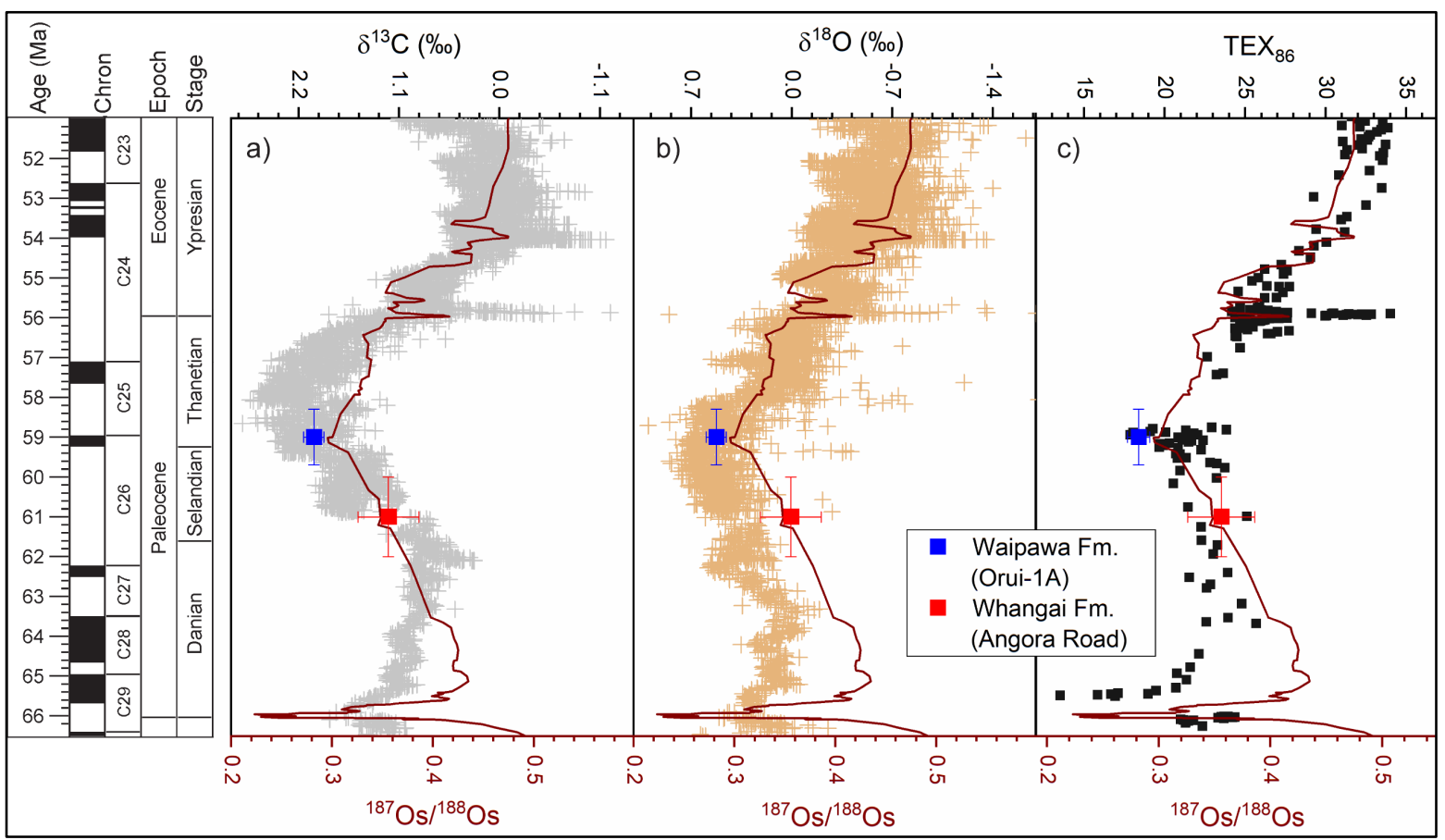

Fig. 3.10. Trends in benthic stable carbon isotope values $\left(\delta^{13} \mathrm{C}\right)(\mathrm{a})$, benthic oxygen isotope values $\left(\delta^{18} \mathrm{O}\right)(\mathrm{b})$ and TEX $\mathrm{X}_{86}$ values $(\mathrm{c})$, overlain by a smoothing of the composite ${ }^{187} \mathrm{Os} /{ }^{188} \mathrm{Os}$ data from Figure 3.9 (brown line). Data were smoothed using Origin Pro 2018 v9.5.1 software, employing the 5-point adjacent averaging method. $\mathrm{TEX}_{86}$ data are from Hollis et al. (2014); benthic foraminiferal $\delta^{18} \mathrm{O}$ and $\delta^{13} \mathrm{C}$ records are from ODP sites 1209,1262 and 1263 (Westerhold et al., 2017, 2018), calibrated to the age model of Westerhold et al. (2017). The ${ }^{187} \mathrm{Os} /{ }^{188} \mathrm{Os}$ data points for Waipawa and Whangai formations are shown by the purple and blue squares, respectively, with the legends shown applying to all plots.

It has been suggested that large quantities of neo-Tethyan (Triassic to Cretaceous) marine sediments were exhumed, oxidised and eroded during the initial stages of Indian-Asian collision causing the late Paleocene to early Eocene shift to lighter $\delta^{13} \mathrm{C}$ values and the coeval global warming via the greenhouse effect (Beck et al., 1995). Using an estimated average ${ }^{187} \mathrm{Re} /{ }^{188} \mathrm{Os}$ value of 517 for organic-rich shales (calculated from a geochemical database of organic-rich sediments from 42 different sources with a bootstrapped $95 \%$ confidence interval of 468 to 582; Dubin and Peucker-Ehrenbrink, 2015) and seawater ${ }^{187} \mathrm{Os} /{ }^{188}$ Os values ranging from $\sim 0.2$ to 0.9 for the Triassic to Cretaceous period (100-250 Ma; Cohen and Coe, 2007; Porter et al., 2013; Dubin and Peucker-Ehrenbrink, 2015; Them et al., 2017; van Acken et al., 2019), these exhumed marine sediments would have contained ${ }^{187} \mathrm{Os} /{ }^{188} \mathrm{Os}$ values ranging from 
1.1 to 3.1 , with an average of 2.1 , significantly more radiogenic than the average ${ }^{187} \mathrm{Os} /{ }^{188} \mathrm{Os}$ value of 1.4 for the upper continental crust. Further, the high abundance of Re and Os in organic-rich sediments coupled with the propensity of the Os hosted in these sediments to be readily mobilized during chemical weathering compared to the Os in typical granitic upper continental crust (Peucker-Ehrenbrink and Hannigan, 2000; Jaffe et al., 2002; PiersonWickmann et al., 2002; Georgiev et al., 2012; Dubin and Peucker-Ehrenbrink, 2015) supports the interpretation that exposure of the neo-Tethyan organic-rich sediments in the late Paleocene may have disproportionately drive seawater ${ }^{187} \mathrm{Os} /{ }^{188} \mathrm{Os}$ to more radiogenic values.

\subsection{Conclusions}

The Re-Os geochronology of the Waipawa Formation has yielded ages of $57.5 \pm 3.5 \mathrm{Ma}$ from the Orui-1A drill hole and $58.3 \pm 6.5 \mathrm{Ma}$ from the Taylor White section that are in close agreement with the available biostratigraphic age range for the formation (59.4 to $58.7 \mathrm{Ma}$; Hollis et al., 2014). These are the first radiometric ages for this formation and demonstrate the potential of the Re-Os method for future dating of correlative units identified in other sedimentary basins across the Southwest Pacific. However, the large uncertainties in the ReOs dates make it difficult to refine the age control and establish whether all known occurrences of the Waipawa Formation are coeval. Future Re-Os studies on the formation should target intervals where the spread in Re-Os ratios is likely to be larger based on variation in organic matter type and other geochemical features, especially in the upper part of the formation. The results from the Taylor White section also highlight the benefit of using fresh core material for such work given the apparent high susceptibility of the Waipawa Formation to oxidative weathering that may disturb the Re-Os system.

The initial ${ }^{187} \mathrm{Os} /{ }^{188}$ Os values for the Waipawa $(\sim 0.28)$ and Whangai $(\sim 0.36)$ formations are non-radiogenic and broadly similar to ${ }^{187} \mathrm{Os} /{ }^{188} \mathrm{Os}$ values from coeval pelagic sediments, further constraining the low-resolution marine Os record for the Paleocene. The broad correlation between the ${ }^{187} \mathrm{Os} /{ }^{188}$ Os record and the global temperature proxies $\left(\delta^{18} \mathrm{O}\right.$, TEX 86$)$ and carbon isotope $\left(\delta^{13} \mathrm{C}\right)$ records from the middle Paleocene to early Eocene suggests that changes in global weathering patterns may have been the main driver of the Os geochemical cycle during this time. 


\section{DEPOSITIONAL INFLUENCES ON RE-OS SYSTEMATICS OF LATE CRetaceous-EOCENE FLUVio-deltaic COALS AND COALY MUDSTONES, TARANAKI BASIN, NEW ZEALAND}

\section{Preface}

$A$ version of this chapter will be submitted to the International Journal of Coal Geology, coauthored by $M H, R S, D S$ and $S N$.

Some of the samples used in this study were provided by RS from a collection of outcrop samples held at GNS Science, Lower Hutt. The rest of the samples were obtained by myself and $S N$ from the National Core store in Featherston, New Zealand. All sample preparations, laboratory analyses, data interpretations and manuscript preparation were undertaken by myself with comments and suggestions from all co-authors. MH, SN, DS and technicians at VUW, GNS Science and Durham University assisted in setting up laboratory protocols and training on instrumentation and data reduction. Sulfur analyses were undertaken by CRL Energy Ltd. 


\subsection{Introduction}

Fluvio-deltaic systems are important components of a sedimentary basin. Their presence, thickness, stacking patterns and geochemical features represent a multi-faceted interplay between changing tectonic regimes, sediment supply and sea-level variations, which is integral to the understanding of the structural and thermal evolution of a basin (Sherwood et al., 1992; Flores and Sykes, 1996; Higgs et al., 2012). Fluvio-deltaic coals and coaly mudstones are also important economically as sources of energy (coal, oil and gas). The late Cretaceous-Eocene coal measures and coaly mudstones of the Taranaki Basin, New Zealand, for example, are the primary source of most the 588.2 million barrels (MMBbls) oil and 1401.9 million barrels of oil equivalent (MMBOE) gas reserves that have been discovered in the basin (Sykes et al., 2004; Sykes and Raine, 2008; New Zealand Petroleum and Minerals, 2014; Energy and Building Trends, 2017). Establishing the depositional ages of these sedimentary successions is essential for basin-wide, regional and global correlations of time-equivalent facies. Generally lacking interbedded igneous units for radiometric dating, biostratigraphy has been the main tool for assigning relative ages to the Taranaki coals and coaly mudstones (Cooper et al., 2004; Raine, 2004; Browne et al., 2008; Crouch and Raine, 2012; Higgs et al., 2012). However, scarcity of fossils for palynological analysis and heterogeneity of coal seams limit precise determinations of the biostratigraphic ages (Browne et al., 2008; Crouch and Raine, 2012; Higgs et al., 2012).

Over the last three decades, the Re-Os isotope system has become established as a powerful tool for directly determining the depositional age for organic-rich sedimentary rocks (e.g., Ravizza et al., 1991; Selby and Creaser, 2005b; Tripathy et al., 2018). Due to the organophilic nature of Re and Os, organic-rich rocks are significant crustal reservoirs of these elements, which are largely sequestered from the water column during deposition (Cohen et al., 1999; Peucker-Ehrenbrink and Ravizza, 2000; Creaser et al., 2002; Selby and Creaser, 2003; Cohen, 2004). The high Re and Os concentrations combined with the $\beta$-decay of ${ }^{187} \operatorname{Re}$ to ${ }^{187} \mathrm{Os}$ allow for precise and accurate radiometric dating of the organic-rich rocks. Previous Re-Os studies have mainly focused on marine black shales where the source of Re and Os is primarily from seawater (e.g., Cohen et al., 1999; Selby et al., 2009; Rooney et al., 2010; Bertoni et al., 2014; Tripathy et al., 2018; Washburn et al., 2019). Only three studies have investigated factors controlling Re-Os systematics and geochronology in coastal and fluvial deposits (Baioumy et 
al., 2011; Tripathy et al., 2015; Goswami et al., 2018). Two of these studies analysed fully terrestrial coals and shales and reported low average Re and Os concentrations $(0.5 \pm 0.4 \mathrm{ppb}$ and $23 \pm 26 \mathrm{ppt}$, respectively) and ${ }^{187} \mathrm{Re} /{ }^{188} \mathrm{Os}$ and ${ }^{187} \mathrm{Os} /{ }^{188} \mathrm{Os}$ ratios that lack isochroneity, suggesting Re-Os geochronology may not be applicable to these types of rocks (Baioumy et al., 2011; Goswami et al., 2018). However, a study of the Matewan coal bed, central Appalachian basin, USA, raised the possibility that the Re-Os geochronometer could be successfully applied to near-shore sedimentary rocks (Tripathy et al., 2015). This study demonstrated that marine-influenced coals may have Re (108 $\pm 16 \mathrm{ppb})$ and Os (516 $\pm 90 \mathrm{ppt})$ concentrations and Re-Os isotope ratios that are a few orders of magnitude higher than those of coals deposited in fully terrestrial environments, and in this case, yielding a relatively precise and accurate depositional age of $325 \pm 14 \mathrm{Ma}\left(\mathrm{Osi}_{\mathrm{i}}=0.86 \pm 0.88 ; \mathrm{MSWD}=12\right)$. It is, however, unclear whether all marine-influenced coals are amenable to Re-Os geochronology and what degree of marine transgression into peat-forming mires is required for substantial sequestration and homogenisation of seawater-derived Re and Os. In addition, it is unclear whether the timing (syn-sedimentary vs diagenetic) of such marine influence plays a role.

In this study, Re and Os concentration and Os isotopic composition, bulk pyrolysis and sulfur data are presented for geochemically well-characterized late Cretaceous-Eocene coals and coaly mudstones from the Taranaki Basin, New Zealand (Fig. 4.1), to investigate factors controlling Re-Os systematics in terrestrial and near-shore sedimentary rocks. These coals and coaly mudstones are from the late Cretaceous Rakopi and North Cape formations, Paleocene Farewell Formation and Eocene Mangahewa Formation. This study explores whether the documented slight to very strong marine influence in these rocks enabled significant uptake of marine Re and Os. In addition, the suitability of fluvio-deltaic sediments to Re-Os geochronometry is further evaluated. 

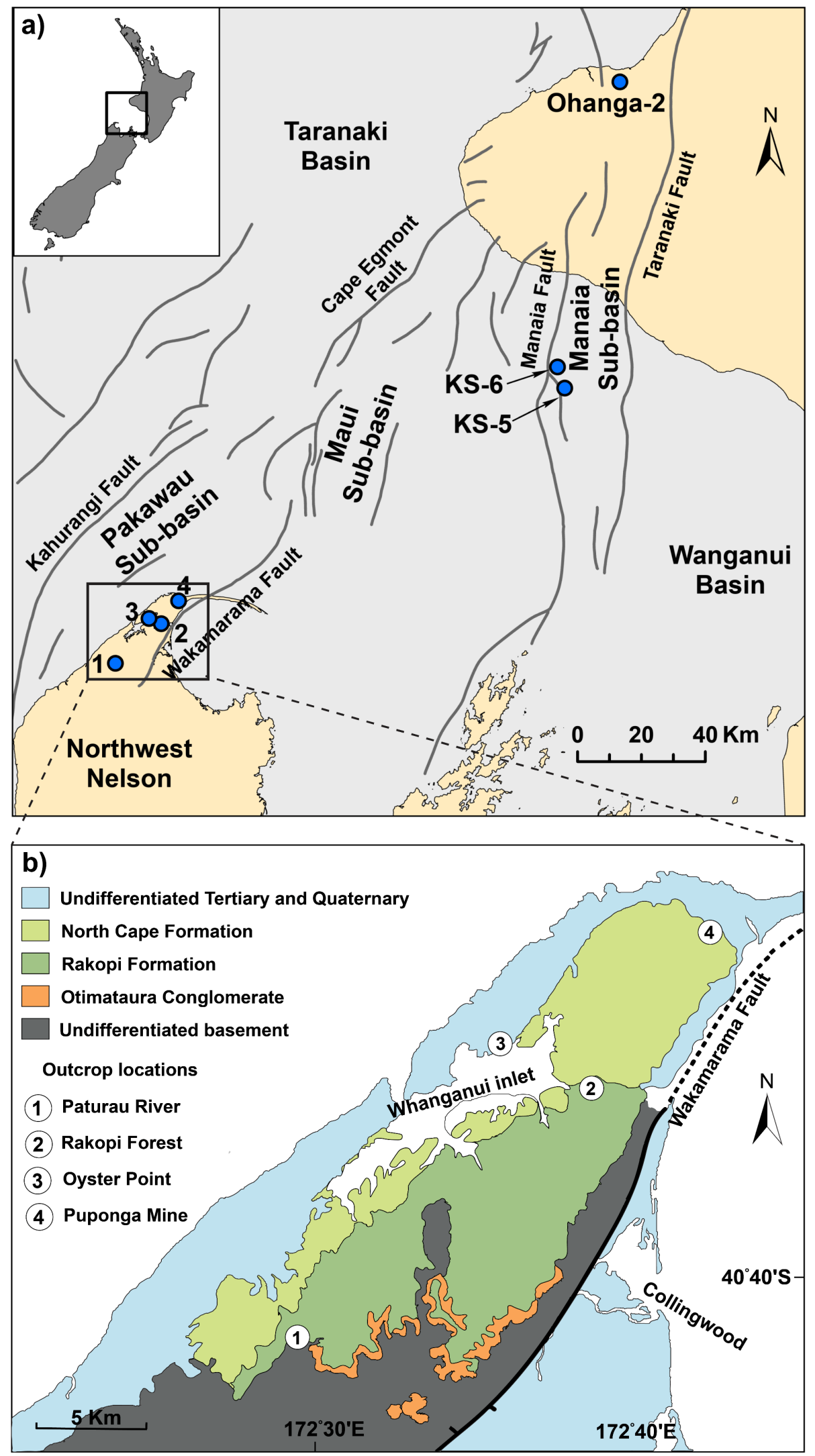

Fig. 4.1. a) Map of the Taranaki Basin showing the main structural features and locations of the studied samples. b) Simplified geological map of the northwest Nelson region (modified after Browne et al., 2008). KS-5 and $-6=$ Kupe South -5 and -6 drillholes. Ohanga- 2 is also a drillhole. 


\subsection{Geological setting}

The Taranaki Basin is a mainly offshore Cretaceous-Cenozoic sedimentary basin located along the west coast of North Island and top of South Island, New Zealand (Fig. 4.1a). At present, the basin is defined by the Taranaki Fault to the east, the Challenger Plateau in the southwest and extends northwest into the Deepwater Taranaki and New Caledonia basins (Uruski, 2008; Strogen et al., 2017). A detailed description of the evolution and stratigraphy of the Taranaki Basin can be found in King and Thrasher (1996). In brief, the basin was formed by rifting during the breakup of eastern Gondwana and opening of Tasman Sea in the Cretaceous period. The basement is dominated by Median Batholith rocks and metasediments of the Buller and Takaka Terrane. In the late Cretaceous, rapid deposition occurred in a series of half-grabens and sub-basins controlled by north to northeast striking normal faults. A passive margin had developed by the end of the Cretaceous which, together with changing sea level, allowed for the deposition of Paleocene-Eocene sediments on coastal plains and an expansive shallow marine shelf (King, 2000).

The Late Cretaceous Rakopi and North Cape formations are terrestrial and marginal marine sedimentary units that were deposited in several isolated and inter-connected depocenters and sub-basins. These formations crop out onshore around the Whanganui Inlet and Puponga area of northwest Nelson (Fig. 4.1b). The Rakopi Formation is the lowest stratigraphic unit in the basin (Fig. 4.2) and consists mainly of sandstone-dominated coal measures that are interbedded with coaly siltstones and mudstones, and with minor conglomerates (Sykes et al., 2004). The formation was deposited in a low-gradient coastal plain environment crossed by a series of rivers and transgressed occasionally by marine incursions (Browne et al., 2008). In the outcrop area, from which all samples studied here were collected, the Rakopi Formation sits on Buller and Takaka Terrane metasedimentary rocks with the Otimataura Conglomerates at its base (Rattenbury et al., 1988). The North Cape Formation overlies the Rakopi Formation and is mainly distinguished from the latter by its greater marine depositional influence (Fig. 4.2; King and Thrasher, 1996). Coal measures are less common in the North Cape Formation than in the Rakopi Formation but are the main lithotype of its Puponga and Wainui members.

The Paleocene Farewell Formation is a fluvio-deltaic unit consisting mainly of sandstones, which are interbedded by coaly mudstones and minor coals. In the vicinity of the Kupe South 
oil and gas field where the samples of this study were taken from, the formation was deposited in a north-draining fluvial system that was controlled by the Manaia Fault, an active fault at the time of deposition (King and Thrasher, 1996). The Farewell Formation generally coarsens upwards in this area, indicating a broadly regressive depositional regime. However, the presence of fining-upwards, sandstone dominated intervals within the formation suggest that there were also transgressive episodes (King and Thrasher, 1996).

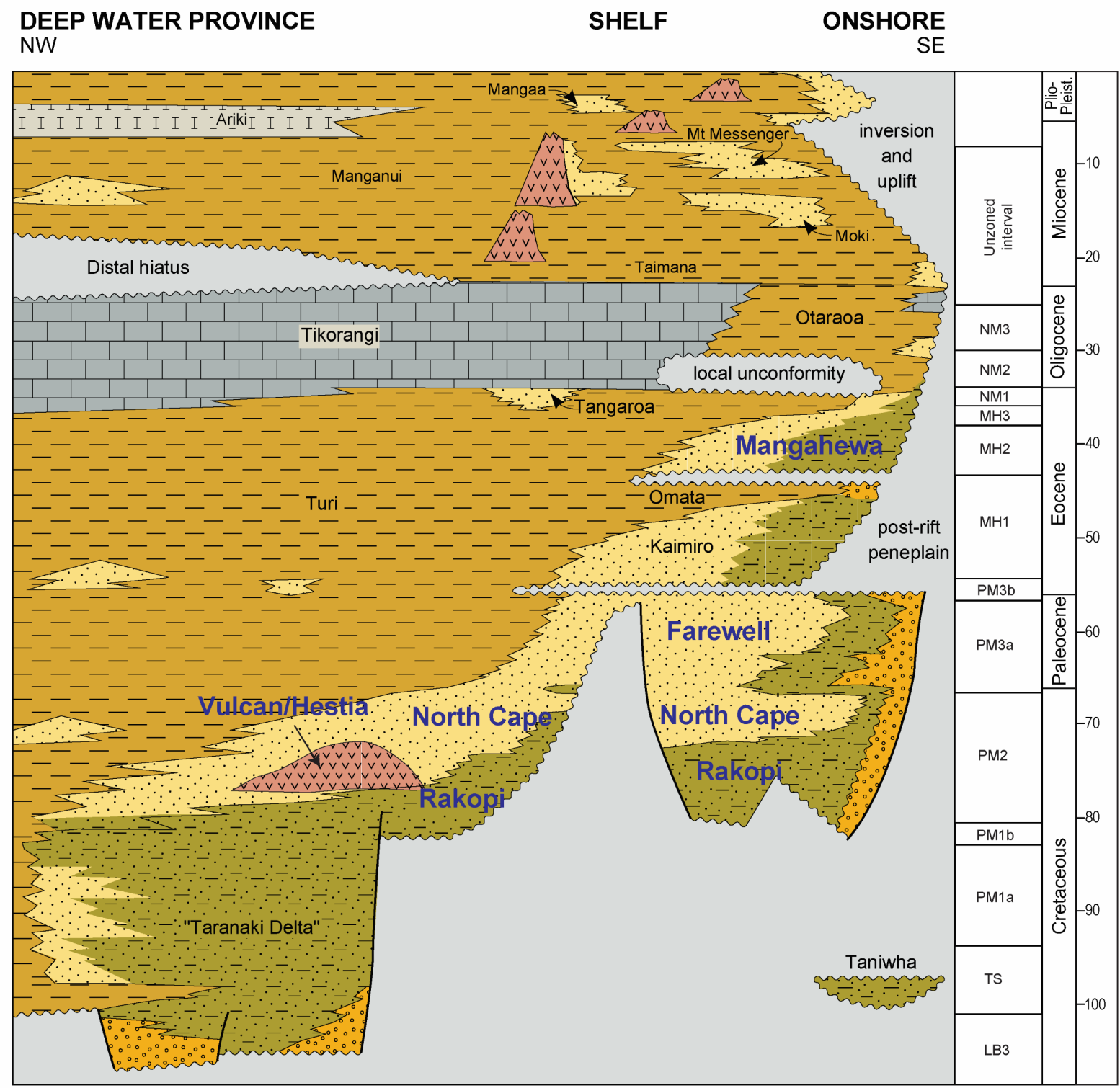

$\vee \vee \vee$
$\vee \vee v$
$\vee \vee v$

Fig. 4.2. Generalized chronostratigraphic panel for the Taranaki Basin across a northwest-southeast transect. Sampled formations are highlighted by bold blue font. The stratigraphic location of the Vulcan/Hestia volcano is from Uruski (2020). Modified from New Zealand Petroleum and Minerals (2014). 
The middle-late Eocene Mangahewa Formation consists of coal measures and marginal marine, shoreline sediments (King and Thrasher, 1996; Sykes et al., 2014). The coal intervals are intermittently developed throughout the formation and are best developed in the southeastern part of the basin where samples of this study were obtained from, becoming thinner and more sporadic towards the north-west, coincident with a changing coastal plain to shallow marine depositional environment (Higgs et al., 2009; 2012). Coal seams are thick and numerous in the upper section of the Eocene succession compared to the lower part, reflecting a change from transgressive to regressive depositional phases (Higgs et al., 2012).

\subsection{Materials and methods}

\subsubsection{Samples}

Eight outcrop samples from the Rakopi and North Cape formations and twenty core samples from the Farewell and Mangahewa formations, Taranaki Basin, New Zealand, were selected for this study. Coaly mudstones, shaly coals, and coals form a continuum with increasing TOC and decreasing mineral content from coaly mudstones to coals (Sykes and Snowdon, 2002). For practical purposes, this continuum can be divided into three broad lithologies using TOC thresholds: coaly mudstones with $\leq 20 \%$ TOC, shaly coals with $20-50 \%$ TOC, and coals with $\geq 50 \%$ TOC (Sykes and Raine, 2008). In the present study, almost all samples have either $\leq 20 \%$ TOC and can, therefore, be classed as coaly mudstones, or $\geq 50 \%$ TOC and can be classed as coals. Two of the samples are more strictly shaly coals with TOC values of $24.2 \%$ and $49.8 \%$ (Tables 4.1 and 4.2), but for simplicity are grouped with the coaly mudstones and coals, respectively.

\subsubsection{Rakopi and North Cape formations}

Samples from the Rakopi and North Cape formations were obtained from a collection of archived outcrop samples held at GNS Science, previously collected from different outcrop locations in the Collingwood district of northwest Nelson (Fig. 4.1b). Prior to sampling, the outer $10-20 \mathrm{~cm}$ of the outcrop was removed to mitigate for potential effects of weathering (Sykes et al., 2004; Sykes and Raine, 2008). The samples showed no visible signs of degeneration from being in storage and had little to no veining or brecciation. Three of the Rakopi samples (PR/1, PR/6 and PR/10) are from a single outcrop on the west bank of the Paturau River, approximately $100 \mathrm{~m}$ downstream from the Thompson Creek (Fig. 4.3; Sykes 
et al., 2004). The outcrop contains a series of 10 closely stacked, gently dipping, thin $(<0.5 \mathrm{~m})$ coal seams within a total thickness of ca. $5 \mathrm{~m}$ (Fig. 4.3). The coals contain bright vitrain (wood) lenses and have sharp, planar contacts with interbedded $\sim 0.1$ to $0.5 \mathrm{~m}$ thick dark brown coaly mudstone and grey-brown siltstones (Sykes et al., 2004). Spore-pollen and dinoflagellate cyst biostratigraphy suggest that the Rakopi Formation outcropping in this location is no older than Upper Campanian ( $\sim 80 \mathrm{Ma})$ at its base and is probably of early-late Maastrichtian $(\sim 72 \mathrm{Ma})$ at its top (Browne et al., 2008). The Paturau River outcrop samples studied here are stratigraphically close to the base of the formation. The fourth Rakopi sample (RF/4) is from an outcrop at a forestry block section, approximately $1 \mathrm{~km}$ south of Pakawau Bush Road. The age of the Rakopi Formation at this locality, as well as its relationship with the overlying North Cape Formation, is uncertain but it is believed to represent the uppermost part of the formation (Browne et al., 2008).

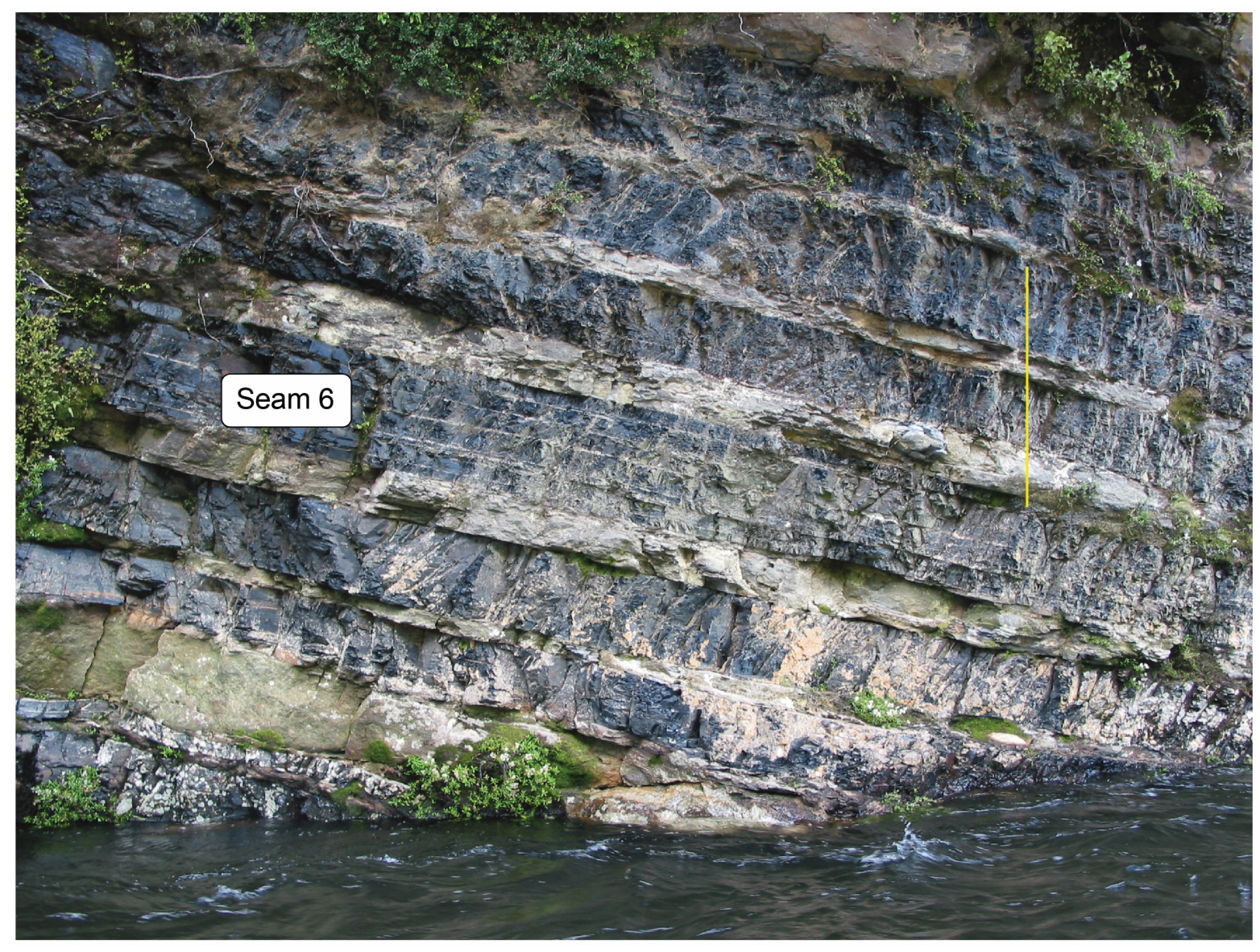

Fig. 4.3. Outcrop of thin, planar mire coal beds and intervening coaly mudstone-siltstone beds within the Rakopi Formation exposed in the upper reaches of the Paturau River (see Fig. 4.1 for location). Seam 6, containing distinctive thin mudstone laminae, and from which sample PR/6 was collected is labelled. Seams 1 and 10 from which samples PR/1 and 10 were collected are out of the field of view. Yellow scale bar is approximately $1 \mathrm{~m}$ long (Sykes et al., 2004). 
Three of the North Cape Formation samples $(\mathrm{PM} / 3 \mathrm{M}, \mathrm{PM} / 3 \mathrm{~N}$, and $\mathrm{PM} / 3 \mathrm{O})$ are from the base of a $2 \mathrm{~m}$ thick full channel coal seam exposed in the adit of the abandoned Puponga coal mine (Fig. 4.1b; Sykes and Raine, 2008). Based on biostratigraphy, this coal seam is estimated to be of upper PM2 zone age (latest Maastrichtian, $67 \mathrm{Ma}$ ), very close to the top of the North Cape Formation (Raine, 2004). The fourth North Cape sample (OP/1) is from a thin, $15 \mathrm{~cm}$ thick coal seam at Oyster Point (Fig. 4.1b). Like the Puponga Mine samples, sample OP/1 is also latest Maastrichtian in age (ca. $67 \mathrm{Ma})$.

\subsubsection{Farewell Formation}

The Farewell samples were obtained from two exploration drillhole cores, Kupe South-5 and Kupe South-6, housed at the National Core Store in Featherston, New Zealand. The Kupe South-5 (3954'55.72" S, 17408'47.51" E) and Kupe South-6 (3951'09.63" S, 174º7'11.09" E) drill-holes are approximately $7 \mathrm{~km}$ apart, in offshore Taranaki Basin (Fig. 4.1a). Both cores were in good condition and the recommended protocols for selecting and sampling organicrich sediments for precise Re-Os geochronology (i.e., a relatively small stratigraphic interval with no alteration or veining and large sample material $[\geq 20 \mathrm{~g}]$ ) were followed (Kendall et al., 2009b; Stein and Hannah, 2014). In the Kupe South-5 core, seven samples were obtained from a $2.7 \mathrm{~m}$ interval between $2911.5 \mathrm{~m}$ and $2914.2 \mathrm{~m}$ (Fig. 4.4a). Five of these samples are closely spaced in a $0.5 \mathrm{~m}$ interval between $2912.8 \mathrm{~m}$ and $2913.3 \mathrm{~m}$ whereas the other two samples were selected to target a slight marine incursion at $2911.5 \mathrm{~m}$ (indicated by rare dinoflagellate cysts) and a $1 \mathrm{~cm}$ thick vitreous coal bed at $2914.2 \mathrm{~m}$ (Fig. 4.4a; Morgans and Pocknall, 1991; Flores et al., 1998; Crouch and Raine, 2012). The sampled interval comprises mainly coaly mudstones and is interpreted to have been deposited in vegetated, low-lying swamps that were prone to influx of clastic sediments during fluvial floods (Flores et al., 1998). Palynological studies indicate that this interval is close to the top of Paleocene (Teurian) PM3a zone and therefore, the Kupe South-5 samples are considered to be late Paleocene in age (i.e. 59.2 to $56.0 \mathrm{Ma}$; Crouch and Raine, 2012). In Kupe South-6, five samples were obtained from a $0.8 \mathrm{~m}$ interval between $3167.2 \mathrm{~m}$ and $3168.0 \mathrm{~m}$ (Fig. 4.4b). This interval comprises coaly mudstones that were deposited in a more distal floodplain lake setting and is also considered to be late Paleocene in age (59.2 to $56.0 \mathrm{Ma}$ ) based on wireline logs (Constantine, 2008). 


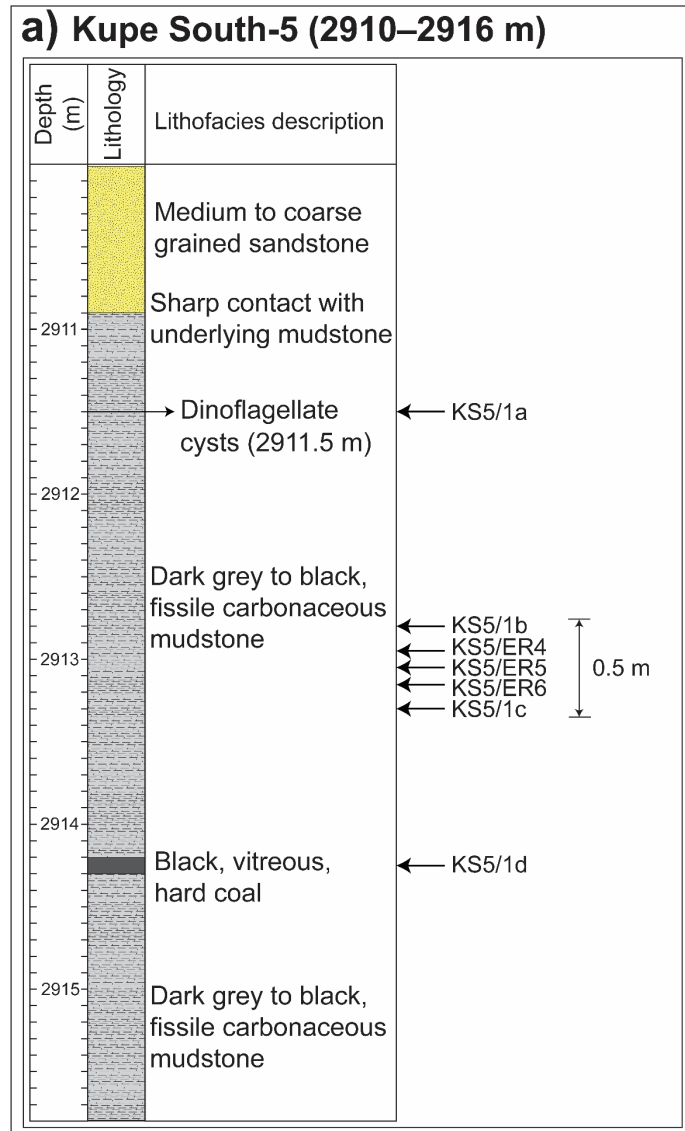

b) Kupe South-6 (3166-3169 m)
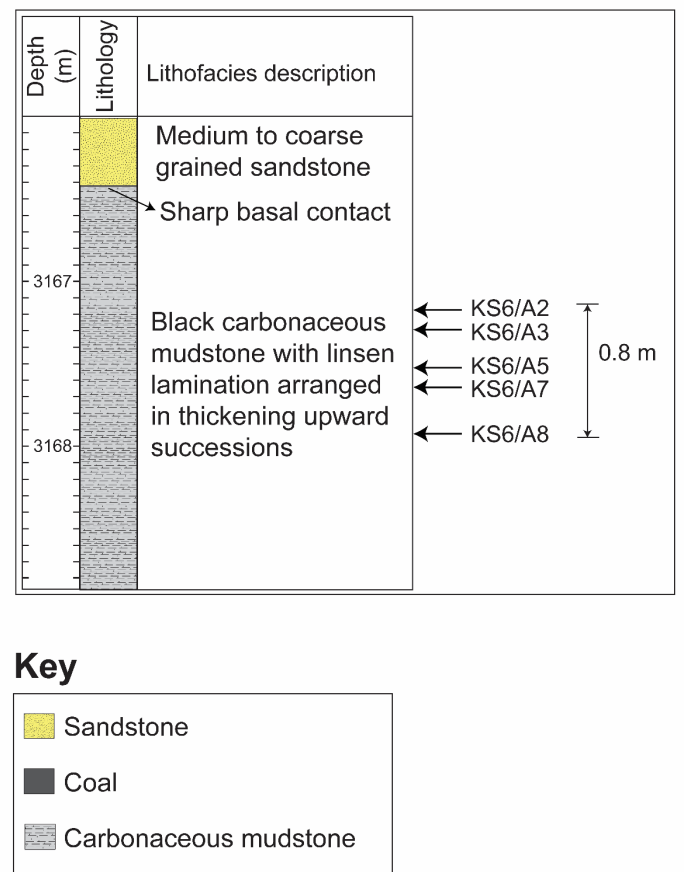

\section{c) Kupe South-5 picture}

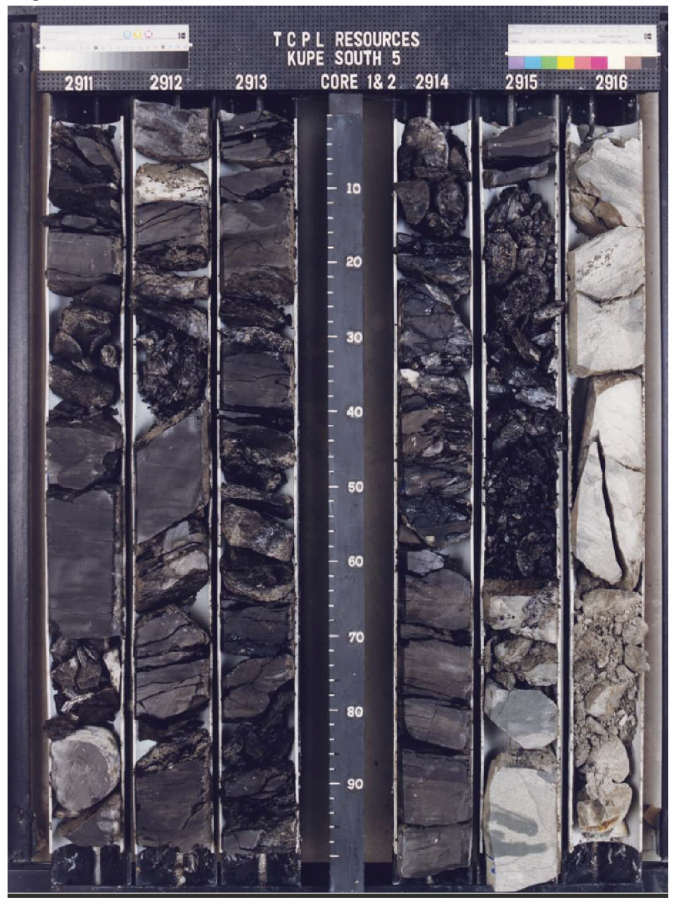

d) Kupe South-6 picture

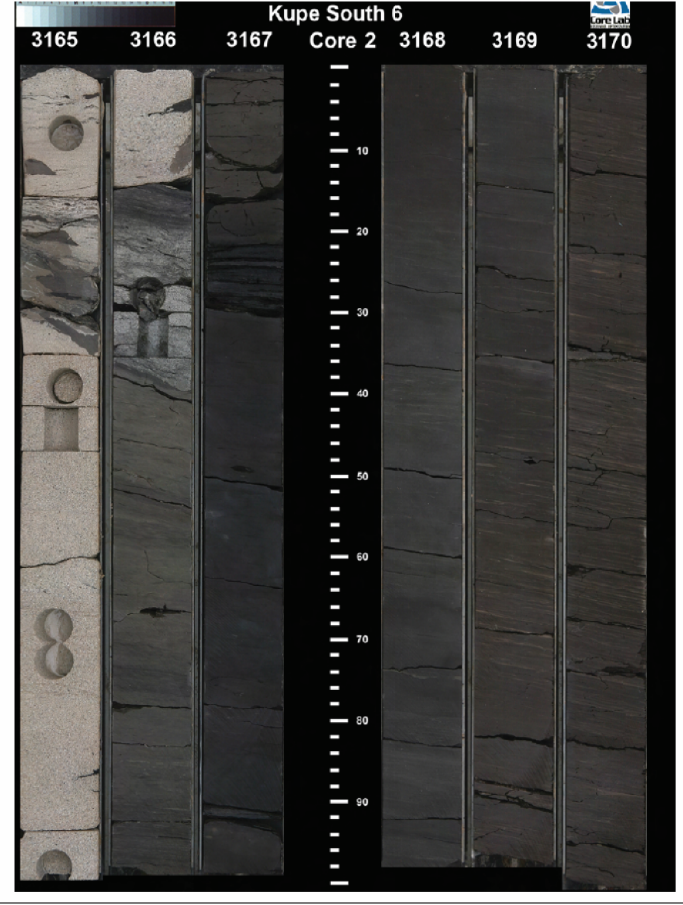

Fig. 4.4. Core description $(a, b)$ and photographs $(c, d)$ of the sampled intervals of the Farewell Formation in Kupe South-5 and -6, respectively. The pictures show that the Kupe South-5 core is very coaly with coarse sediments compared to the sampled interval in Kupe South-6, which is laminated and lacks coarse sediments. 


\subsubsection{Mangahewa Formation}

Samples from the Mangahewa Formation were obtained from archived core samples held at GNS Science, previously obtained from three, closely spaced, isorank coal seams (named seam 10, 11 and 12) from the Ohanga-2 drill-hole core (Fig. 4.5; Sykes et al., 1999). The Ohanga-2 drill-hole is located onshore Taranaki basin (3901'43.43" S, 174²1'07.32" E; Fig. 4.1a). The three seams cover a total stratigraphic thickness of $5 \mathrm{~m}$, with seams 10 and 11 being 0.39 and $0.91 \mathrm{~m}$ thick, respectively. Seam 12 is estimated from electric logs to have a thickness of $1 \pm$ $0.15 \mathrm{~m}$, however, only $0.67 \mathrm{~m}$ was recovered because of jamming of the core barrel (Murray and McGregor, 1998; Sykes et al., 1999). The two upper coals seams (10 and 11) differ from the lower seam (12) in their chemical and petrographic properties, the most prominent of which is a strong marine influence in the two upper seams, as reflected by higher sulfur contents and microfossils (Fig. 4.5a; Sykes et al., 1999). In total, six coals from the three seams and two coaly mudstones that border the lower and upper part of seam 10 were selected for this study. The Mangahewa Formation samples are stratigraphically located towards the top of the Mangahewa Formation and are estimated to be latest Eocene in age (37 Ma; Murray and McGregor, 1998).

\subsubsection{Analytical methods}

\subsubsection{Bulk pyrolysis and sulfur analyses}

For most of the Rakopi, North Cape and Mangahewa samples, bulk pyrolysis and sulfur data had previously been obtained by GNS Science and are publicly available in a geochemical database and related reports (e.g., Sykes et al., 2012) from its Petroleum Basin Explorer (PBE) online data portal (https://data.gns.cri.nz/pbe/). The total sulfur (TS) data were used together with palynological information (Sykes et al., 1999; 2004; Browne et al., 2008) to target intervals within the three formations that have varying degrees of marine influence. Total organic carbon (TOC) and Rock-Eval data for all Farewell Formation samples (except KS5/1a, b, c and d) were obtained in this study following protocols outlined in Naeher et al. (2019). TS content for all the Farewell Formation samples (except KS5/1d), as well as samples PM/3M, Oh2/MM10a and Oh2/MM10i, were also obtained in this study by CRL Energy Ltd, following standard procedures that were used previously for all other samples - ASTM D4239 for TS (ASTM International, 2018) and AS1038.11-2002 for forms of sulfur (Standards Australia, 2002). Forms of sulfur (organic, pyritic and sulfate) were determined for the Mangahewa 
samples to provide more information about the source and processes of sulfur incorporation in these samples.

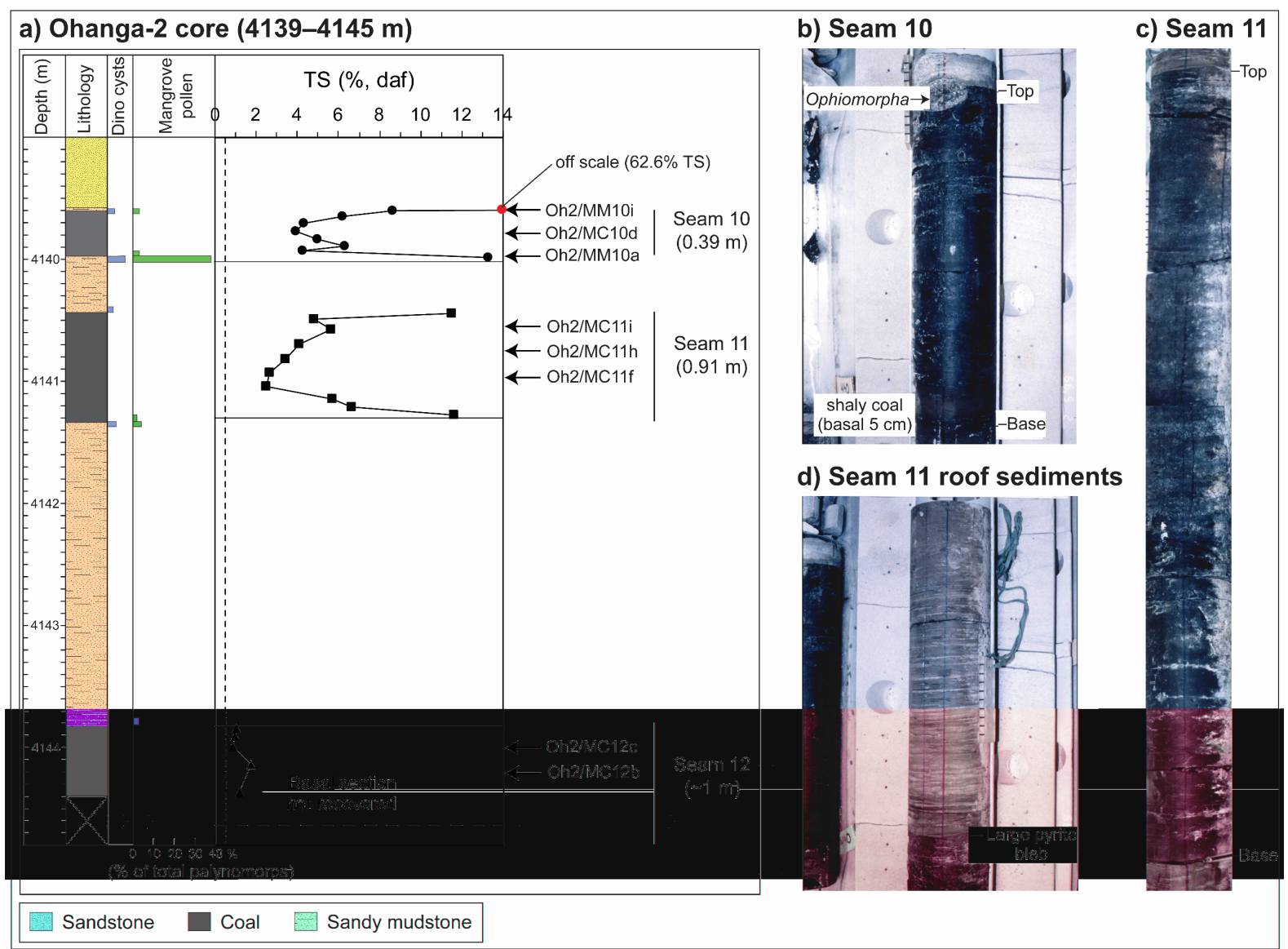

Fig. 4.5. a) Core description of the sampled interval of the Mangahewa Formation in Ohanga-2 well showing coal seams 10,11 and 12 (modified after Sykes et al., 1999). High sulfur concentrations are recorded in intervals containing dinoflagellate cysts and mangrove pollen, indicating syn-sedimentary (rather than late diagenetic) emplacement of sulfur as a result of marine influence. The vertical dashed line in the sulfur panel is at $0.5 \%$ total sulfur (TS; dry ash-free [daf]), which is the inferred approximate upper limit for entirely freshwater coals (e.g., Sykes et al., 2004; 2014). Also shown are photographs of b) coal seam 10, c) coal seam 11 and d) the roof sediments of seam 11. An Ophiomorpha burrow in the roof of seam 10, a large pyrite bleb at the top of seam 11 and inferred tidal-laminated sediments directly overlying seam 11 are clear evidence of marine influence in the coal-forming environment.

\subsubsection{Re-Os analysis}

In preparation for Re-Os analysis, all samples were processed at Victoria University of Wellington following the protocols of Kendall et al. (2009b). The samples were polished to remove cutting and drilling marks, dried in an oven overnight at $40{ }^{\circ} \mathrm{C}$, broken into small chips with no metal contact, and crushed to a fine powder $(\sim 30 \mu \mathrm{m})$ using an agate mill. Re-Os analyses were carried out at the Laboratory for Source Rock and Sulfide Geochemistry and 
Geochronology, Durham University, UK. The Farewell samples were digested and purified using established protocols for Re-Os geochronology in sedimentary rocks (e.g., Rooney et al., 2011; Jones et al., 2018 and references therein). In brief, $\sim 1 \mathrm{~g}$ of powdered rock was spiked with a known amount of ${ }^{190} \mathrm{Os}+{ }^{185} \mathrm{Re}$ mixed tracer solution and digested with $\mathrm{Cr}^{\mathrm{VI}}-\mathrm{H}_{2} \mathrm{SO}_{4}$ in a sealed Carius tube at $220^{\circ} \mathrm{C}$ for $48 \mathrm{~h}$. Os was separated and purified using chloroform solvent extraction and $\mathrm{HBr}$ micro-distillation methods whereas $\mathrm{Re}$ was purified using $\mathrm{NaOH}$-acetone solvent extraction and anion exchange chromatography. The $\mathrm{Cr}^{\mathrm{VI}}-\mathrm{H}_{2} \mathrm{SO}_{4}$ solution digestion method is preferred for Re-Os analysis because it preferentially liberates hydrogenous Re and Os (Selby and Creaser, 2003; Rooney et al., 2011). However, digestion with the $\mathrm{Cr}^{\mathrm{VI}}-\mathrm{H}_{2} \mathrm{SO}_{4}$ solution appeared to be incomplete for most of the samples from the Mangahewa, North Cape and Rakopi formations, based on the green colour of partially reduced $\mathrm{Cr}^{\mathrm{III}}$ species in the digest, very weak analyte signal on the mass spectrometer and negative Os isotope compositions for some of the samples, which is a likely indicator of inadequate spike-sample equilibration. Increasing the acid to sample ratio by up to a factor of three did not improve the digestion outcome. As a result, all samples from the Rakopi, North Cape and Mangahewa formations (except sample Oh2/MM10i) were digested using a stronger oxidizing solution, inverse aquaregia ( $\left.3 \mathrm{ml} \mathrm{HCl}: 6 \mathrm{ml} \mathrm{HNO}_{3}\right)$. It has been shown for moderately low TOC marine samples that the inverse aqua-regia digestion method can yield Re and Os concentrations, Re-Os ages and Osi ratios that are identical within uncertainty to those produced using the $\mathrm{Cr}^{\mathrm{VI}}-\mathrm{H}_{2} \mathrm{SO}_{4}$ method, albeit with lower ${ }^{187} \mathrm{Re} /{ }^{188} \mathrm{Os}$ and ${ }^{187} \mathrm{Os} /{ }^{188} \mathrm{Os}$ ratios (15\% and $9 \%$ lower, respectively) and lower precision in the Re-Os ages, which has been attributed to incorporation of detrital Os component (Selby and Creaser, 2003; Kendall et al., 2004; Rooney et al., 2011). Given that the Rakopi, North Cape and Mangahewa samples were not specifically selected for Re-Os geochronology and that the mineral component in these samples would likely be low due to their relatively high TOC content (average of 59.3\%), it is considered that any nonhydrogenous contribution to the Re-Os analysis by employing the inverse aqua-regia digestion method would have been minimal and would not affect the objectives of this study.

In the inverse aqua-regia method, $200 \mathrm{mg}$ of powdered rock was spiked with a known amount of ${ }^{190} \mathrm{Os}$ and ${ }^{185} \mathrm{Re}$ mixed tracer solution and digested with an inverse aqua-regia solution in a sealed Carius tube at $220^{\circ} \mathrm{C}$ for $24 \mathrm{~h}$. Digestion of larger amounts of sample powder (up to 1 g) for improved analytical precision was attempted, however, $200 \mathrm{mg}$ proved to be the safe 
limit to avoid rupturing the Carius tube during digestion. Separation and purification of Re and Os followed similar procedures to those outlined for the Farewell Formation samples.

The purified Re and Os fractions from all samples were loaded onto nickel and platinum filaments, respectively (Selby, 2007). The Re and Os isotopic measurements were then obtained using a Thermo Scientific TRITON Negative Thermal Ionisation Mass Spectrometer housed at the Arthur Holmes Laboratory, Durham University. Rhenium measurements were obtained via static Faraday collection mode whereas ion-counting on a secondary electron multiplier (SEM) was used for Os measurements. Total procedural blanks for the $\mathrm{Cr}^{\mathrm{VI}}-\mathrm{H}_{2} \mathrm{SO}_{4}$ method were $15.2 \pm 0.2 \mathrm{pg}$ Re and $0.45 \pm 0.01 \mathrm{pg}$ Os, with an ${ }^{187} \mathrm{Os} /{ }^{188} \mathrm{Os}$ value of $0.23 \pm 0.06$ $(\mathrm{n}=4)$. Total procedural blanks for the inverse aqua-regia method were $2.3 \pm 0.02 \mathrm{pg} \operatorname{Re}$ and $0.19 \pm 0.01 \mathrm{pg}$ Os, with an ${ }^{187} \mathrm{Os} /{ }^{188} \mathrm{Os}$ value of $0.28 \pm 0.17(\mathrm{n}=4)$. Instrument reproducibility and data quality were monitored by repeated measurement of the Durham Romil Osmium Standard (DROsS) solution, which yielded an average ${ }^{187} \mathrm{Os} /{ }^{188} \mathrm{Os}$ of $0.16067 \pm 0.00036(\mathrm{n}=$ $8)$, in good agreement with values reported by other laboratories $(0.16078 \pm 0.00024$, Liu and Pearson, 2014; 0.16091 \pm 0.00015 , van Acken et al., 2019). Uncertainties for the reported ${ }^{187} \mathrm{Re} /{ }^{188} \mathrm{Os}$ and ${ }^{187} \mathrm{Os} /{ }^{188} \mathrm{Os}$ ratios were determined by error propagation of the uncertainties in mass spectrometer measurements, tracer solution calibrations, blank concentrations and isotopic compositions, and the reproducibility of standard Re and Os isotopic ratios.

\subsubsection{Multivariate analysis}

Multivariate analysis was used to assess statistical relationships between Re-Os data and bulk geochemical parameters. Hierarchical cluster analysis (HCA) and principal component analysis (PCA) were applied to 10 parameters from the entire sample set using the Pirouette ${ }^{\circledR}$ (Infometrix Inc.) statistical software. This analysis employed autoscale pre-processing (HCA and PCA), Euclidean metric distance, and incremental linkage (HCA) approaches. In autoscaling, the data for each parameter is mean-centred and their variance scaled. This step standardises all parameters so that they exhibit an equal weighting in the chemometric analysis. The incremental linkage approach was used because it tends to increase distances, and thus, provides a better distinction between clusters (Naeher et al., 2019). 


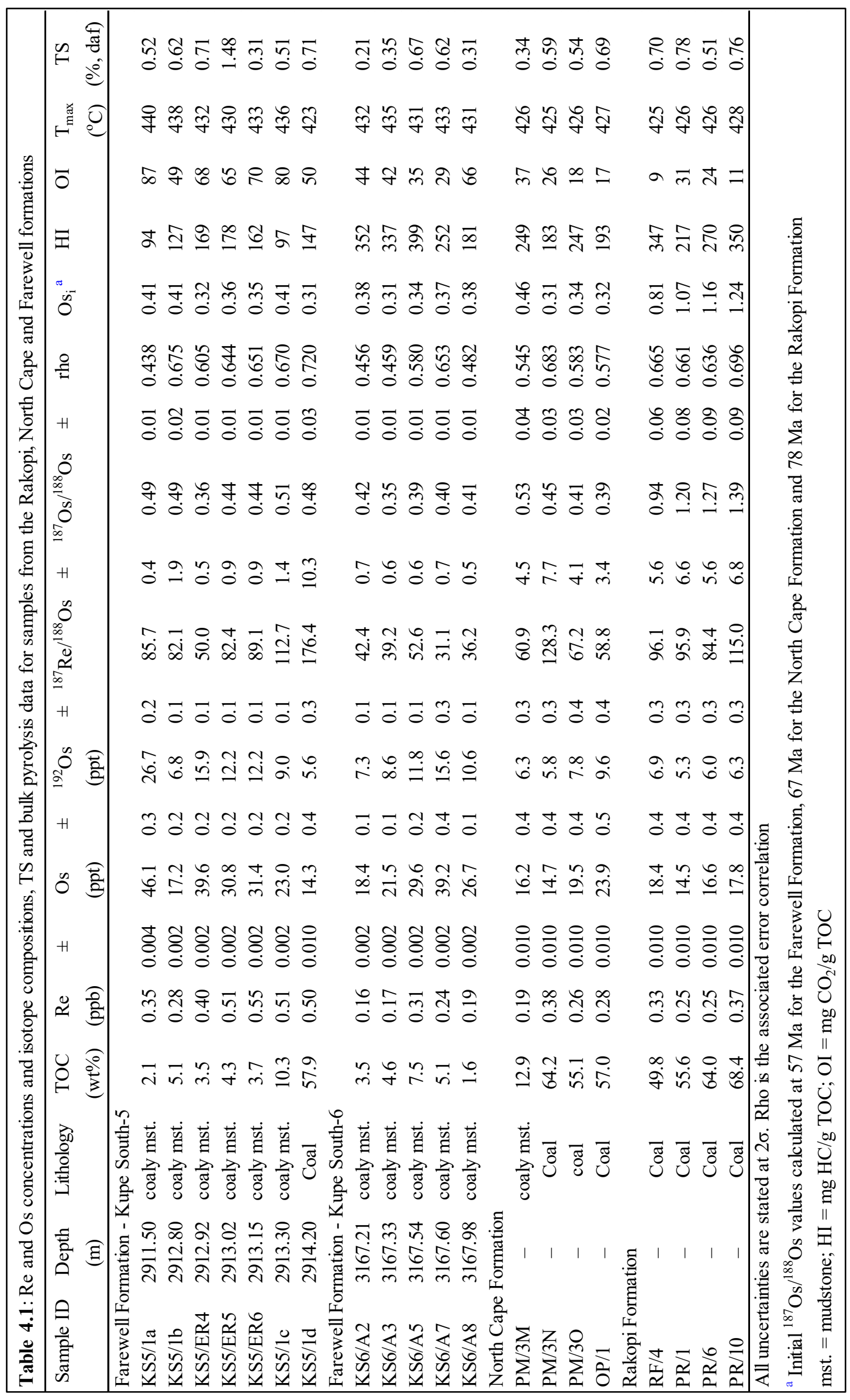




\begin{tabular}{|c|c|c|c|}
\hline 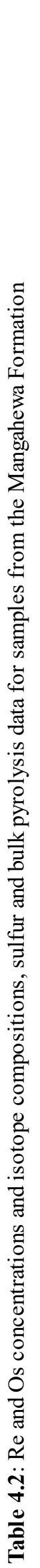 & 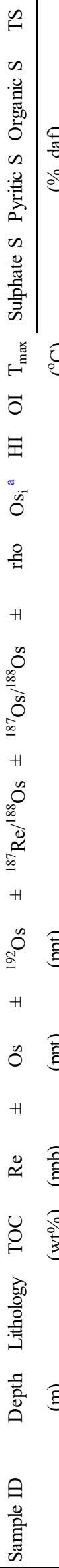 & 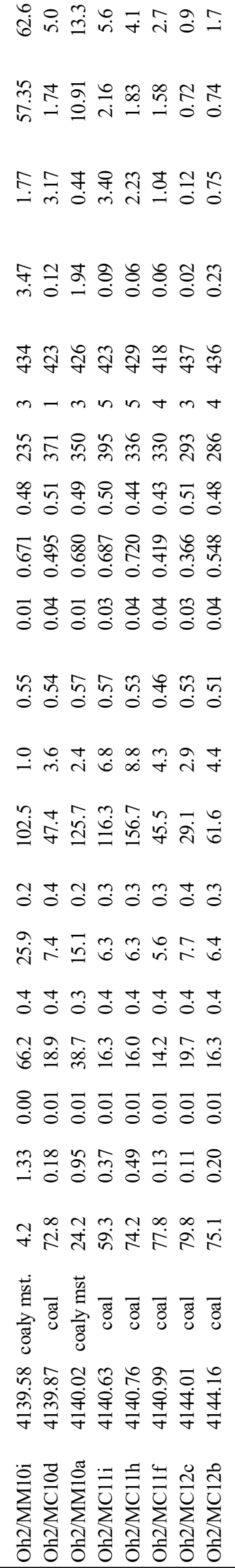 & 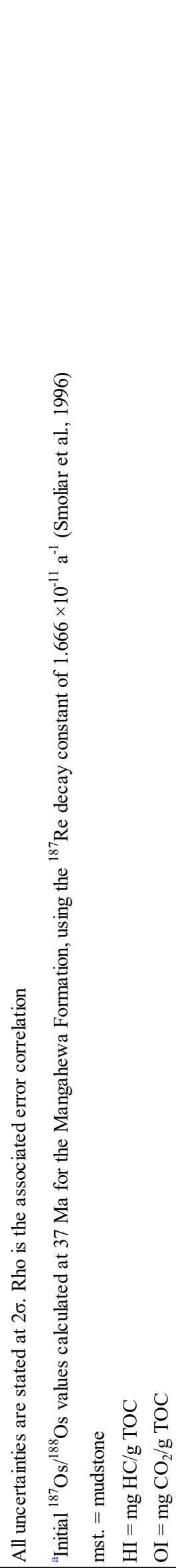 \\
\hline
\end{tabular}




\subsection{Results}

The bulk pyrolysis, TS, sulfur species, Re and Os data for all samples are provided in Tables 4.1 and 4.2. The bulk pyrolysis data are used to evaluate the thermal maturity and type of the organic matter present in the samples (Espitalié et al., 1977; Peters, 1986). TOC content varies widely in samples from the four formations (Fig. 4.6a), reflecting the two different lithologies present i.e. coaly mudstones $(\leq 20 \mathrm{wt} \%)$ and coals $(\geq 50 \mathrm{wt} \%)$. Like TOC, hydrogen index (HI) varies widely in samples from the four formations (94-399 mg HC/g TOC; Fig. 4.6a), indicating variation in either type or thermal maturity of the kerogen. The latter is unlikely given that $\mathrm{T}_{\max }$ values (temperature of maximum pyrolysis yield; $418-440{ }^{\circ} \mathrm{C}$ ) and inferred vitrinite reflectance $\left(0.5\right.$ to $\left.0.7 \% \mathrm{R}_{\mathrm{o}}\right)$ indicate that the kerogen is immature to marginally mature in all samples (Fig. 4.6d, e).

Oxygen index (OI), which is a proxy for the amount of oxygen present in the kerogen (Peters, 1986), is very low in the Mangahewa Formation samples, ranging from $1-5 \mathrm{mg} \mathrm{CO}_{2} / \mathrm{g}$ TOC compared to $9-87 \mathrm{mg} \mathrm{CO} / \mathrm{g}$ TOC in samples from the other three formations (Fig. 4.6b, c). The OI values for the Kupe South-5 samples are highest in the sample set (49-87 mg CO $2 / \mathrm{g}$ TOC) and correlate positively with $\mathrm{T}_{\max }$ values (except for KS5/1b, Fig, 4.6c). Based on the modified van Krevelen diagram (OI vs HI; Fig. 4.6c) and HI vs $T_{\max }$ (Fig. 4.6d) plot, the Farewell samples from Kupe South-5 contain mainly Type III kerogen whereas those from Kupe South-6, as well as those from the Rakopi, North Cape and Mangahewa formations, contain mixed Type II and III kerogen. The low thermal maturity of these samples suggests that the observed kerogen types are representative of the initial organofacies composition.

TS content ranges from 0.2 to $1.5 \%$, daf, in samples from the Rakopi, North Cape and Farewell formations (Fig. 4.6f), indicating entirely terrestrial to slightly marine-influenced depositional conditions. In contrast, TS contents range to considerably higher values in the Mangahewa Formation samples (0.9-62.6, daf; Fig. 4.6f), indicating slight to very strong marine influence. Organic sulfur is very high in the two Mangahewa Formation coaly mudstones compared to pyritic and sulfate sulfur (Table 4.2). In contrast, pyritic sulfur in the Mangahewa Formation coals is relatively high compared to the other forms of sulfur (except for Oh2/MC12c; Table $4.2)$. 

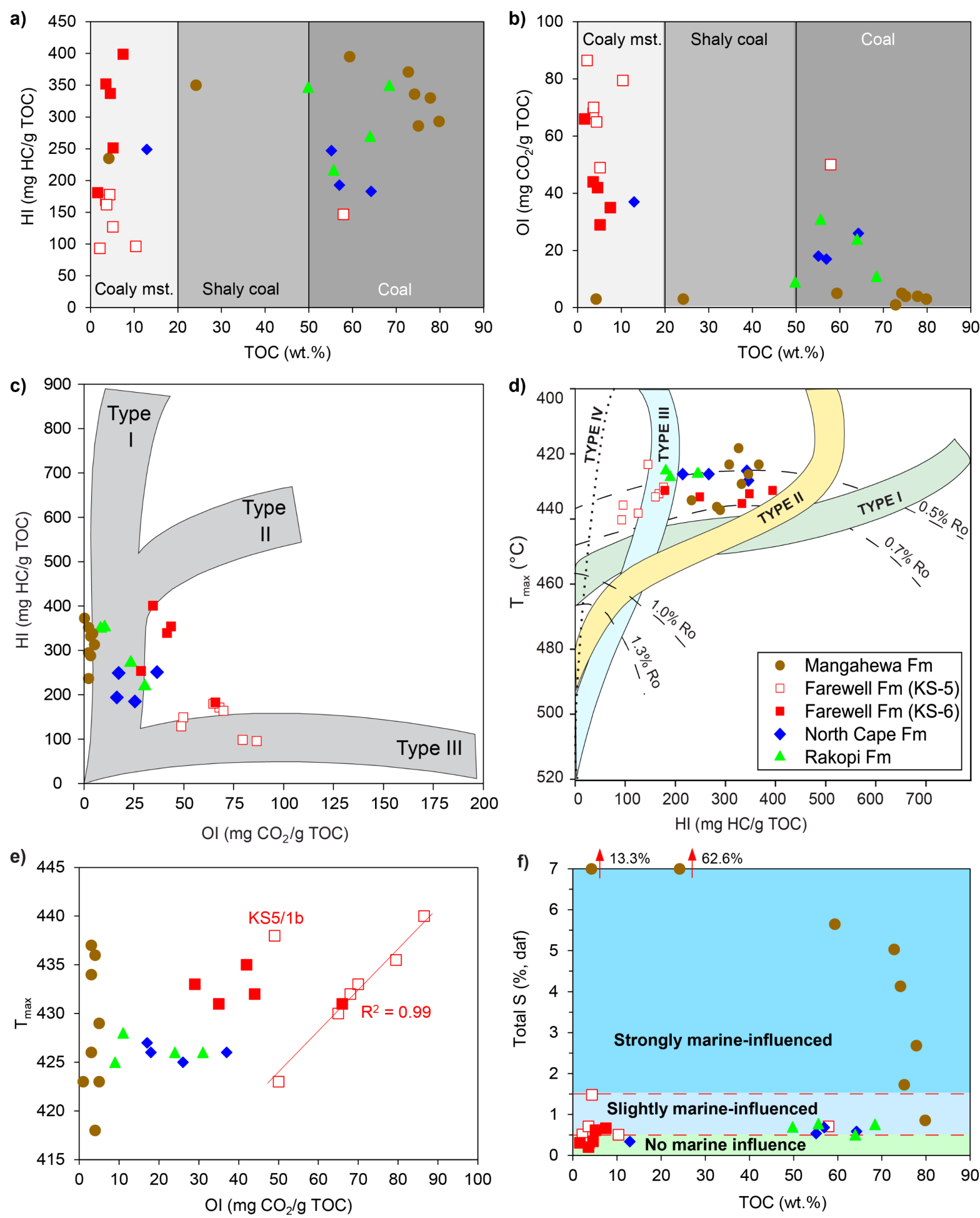

Fig. 4.6. Cross-plots of a) TOC vs HI, b) TOC vs OI, c) OI vs HI, d) HI vs $T_{\max }$, e) OI vs $T_{\max }$, and f) TOC vs TS, for coaly rocks in the sampled formations. TOC thresholds for coaly lithologies are from Sykes and Raine (2008). TS thresholds for the degree of marine-influence are from Sykes $(2001,2004)$. In d), the trends in vitrinite reflectance $\left(\mathrm{R}_{\mathrm{O}}\right)$ and kerogen types are from Peters (1986) and the p:IGI (program for Integrated Geochemical Interpretation) software. In f), samples Oh2/MM10a (13.3\%S) and Oh2/MM10i $(62.6 \% \mathrm{~S})$ are off scale. The legend in d) is used for all plots. KS-5 and $-6=$ wells Kupe South-5 and -6 . 
Re and Os concentrations in all samples range from 0.11 to $1.33 \mathrm{ppb}$ and 14.2 to $66.2 \mathrm{ppt}$ (5.3$26.7 \mathrm{ppt}{ }^{192} \mathrm{Os}$ ), respectively (Fig. 4.7). These concentration ranges are comparable to those reported for the UCC (0.2-2 ppb Re and 30-50 ppt Os; Esser and Turekian, 1993; PeuckerEhrenbrink and Jahn, 2001; Sun et al., 2003) and fully terrestrial coals and shales (0.1-1.4 ppb Re and 8-104 ppt Os; Baioumy et al., 2011; Goswami et al., 2018). There is no relationship between TOC content and Re and ${ }^{192} \mathrm{Os}$ (an estimate of Os chelated at the time of deposition i.e. without radiogenic ingrowth of ${ }^{187} \mathrm{Os}$ ) concentrations across the entire sample set, but strong negative correlations exist between TOC and $\mathrm{Re}\left(\mathrm{R}^{2}=0.94\right)$ and ${ }^{192} \mathrm{Os}\left(\mathrm{R}^{2}=0.89\right)$ concentrations for the Mangahewa Formation samples (Fig. 4.7a, b). Like TOC, there are no simple correlations between TS content and Re and ${ }^{192}$ Os concentrations in samples from the Rakopi, North Cape and Farewell formations (Fig. 4.7c, d). However, the Mangahewa Formation samples show strong correlations between TS and $\mathrm{Re}\left(\mathrm{R}^{2}=0.76\right)$ and ${ }^{192} \mathrm{Os}\left(\mathrm{R}^{2}=\right.$ 0.91) concentrations (Fig. 4.7c, d). The two Mangahewa Formation samples with the highest TS content (Oh2/MM10a [13.3\%, daf] and Oh2/MM10i [62.6\%, daf]) exhibit the highest Re (0.95 and $1.33 \mathrm{ppb}$, respectively) and ${ }^{192} \mathrm{Os}$ (15.1 and $25.9 \mathrm{ppt}$, respectively) concentrations.

The ${ }^{187} \mathrm{Re} /{ }^{188}$ Os values are very low for all samples $(29.1-176.4)$, similar to published ${ }^{187} \mathrm{Re} /{ }^{188}$ Os values for fully terrestrial coals (Baioumy et al., 2011; Goswami et al., 2018), but significantly lower than those reported for the marine-influenced Matewan coals $\left({ }^{187} \mathrm{Re} /{ }^{188} \mathrm{Os}\right.$ = 3299-5134; Tripathy et al., 2015). The Farewell Formation samples from Kupe South-6 exhibit notably lower ${ }^{187} \mathrm{Re} /{ }^{188}$ Os values (31.1-52.6), compared to those from the Kupe South5, which range from 50.0 to 176.4 . The present-day ${ }^{187} \mathrm{Os} /{ }^{188} \mathrm{Os}$ values for samples from the North Cape, Farewell and Mangahewa formations are moderately radiogenic (0.35-0.57), whereas those from the Rakopi Formation are radiogenic (0.94-1.39).

The results of the PCA was used to assess statistical relationships between bulk geochemical parameters (TOC, HI, OI and TS) and Re and Os concentrations and ${ }^{187} \mathrm{Re} /{ }^{188} \mathrm{Os}$ ratios for samples from the four formations (Fig. 4.8). In this analysis, PCA Factor 1 accounted for $45.0 \%$ of the variance while Factor 2 accounted for 30.6\%, showing a trend of changing redox conditions and marine influence within the samples (Fig. 4.8). The PCA score plot (Fig. 4.8a) indicates that Farewell Formation samples were deposited in the most oxidising conditions whereas the Mangahewa Formation samples were deposited in the most reducing conditions. The Re and Os concentrations and ${ }^{187} \mathrm{Re} /{ }^{188} \mathrm{Os}$ ratios appear to be independent of these 
changing redox conditions, consistent with previous findings (Selby et al., 2009; Chapter 3 of this thesis). Rhenium and Os concentrations have a positive loading on Factor 1 and are mainly associated with TS content, which is an indicator of the degree of marine influence.
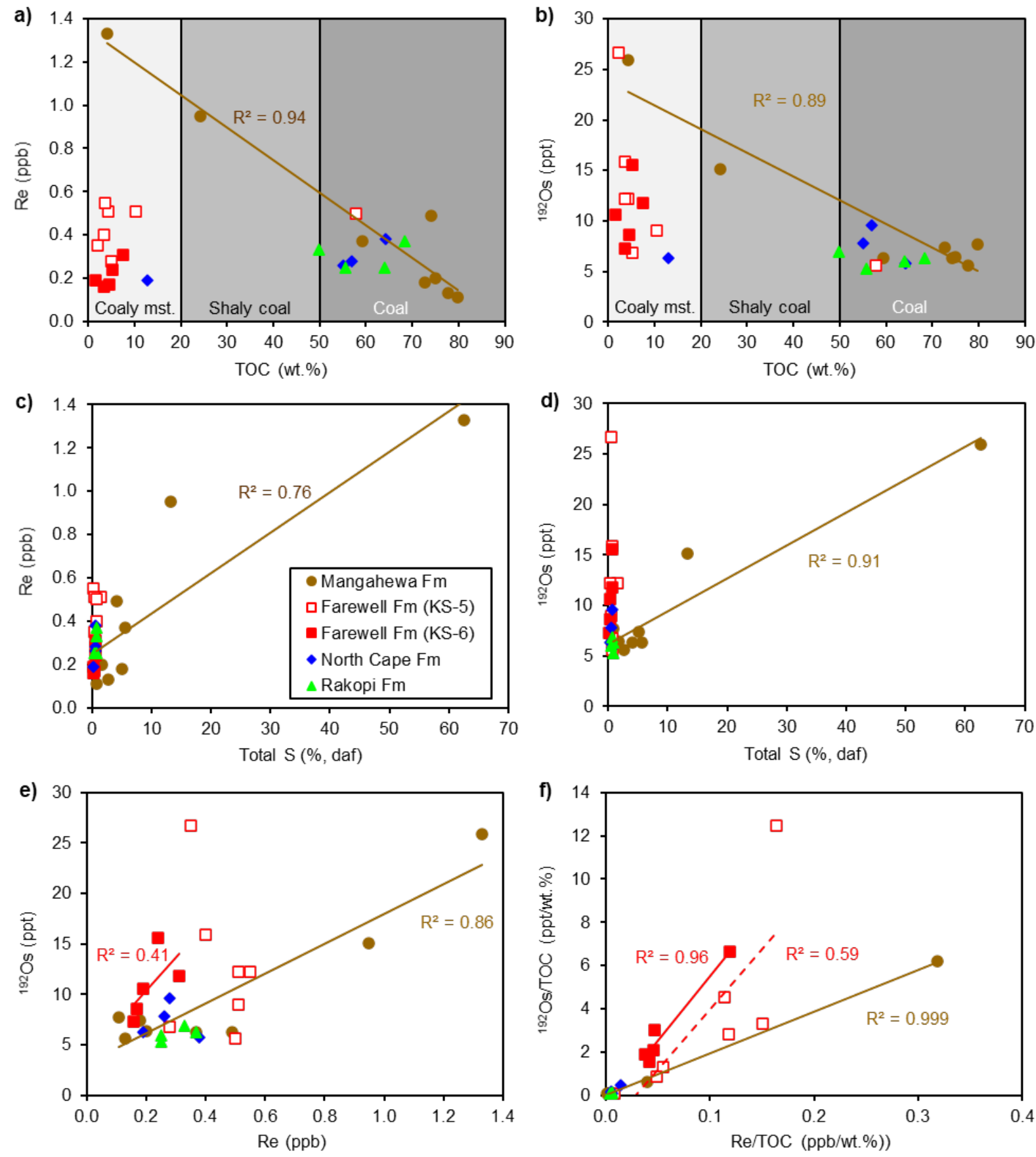

Fig. 4.7. Cross-plots of a) TOC vs Re, b) TOC vs ${ }^{192} \mathrm{Os}$, c) TS vs Re, d) TS vs ${ }^{192} \mathrm{Os}$, e) Re vs ${ }^{192} \mathrm{Os}$ and f) $\mathrm{Re} / \mathrm{TOC}$ vs ${ }^{192} \mathrm{Os} / \mathrm{TOC}$ for coaly rocks in the sampled formations. KS-5 and -6 = wells Kupe South5 and -6 . TOC thresholds for coaly lithologies are from Sykes and Raine (2008). In c) and d), the two Mangahewa Formation samples with the highest TS content (Oh2/MM10a and Oh2/MM10i) exhibit the highest concentrations of $\mathrm{Re}$ and ${ }^{192} \mathrm{Os}$, respectively. 
a)

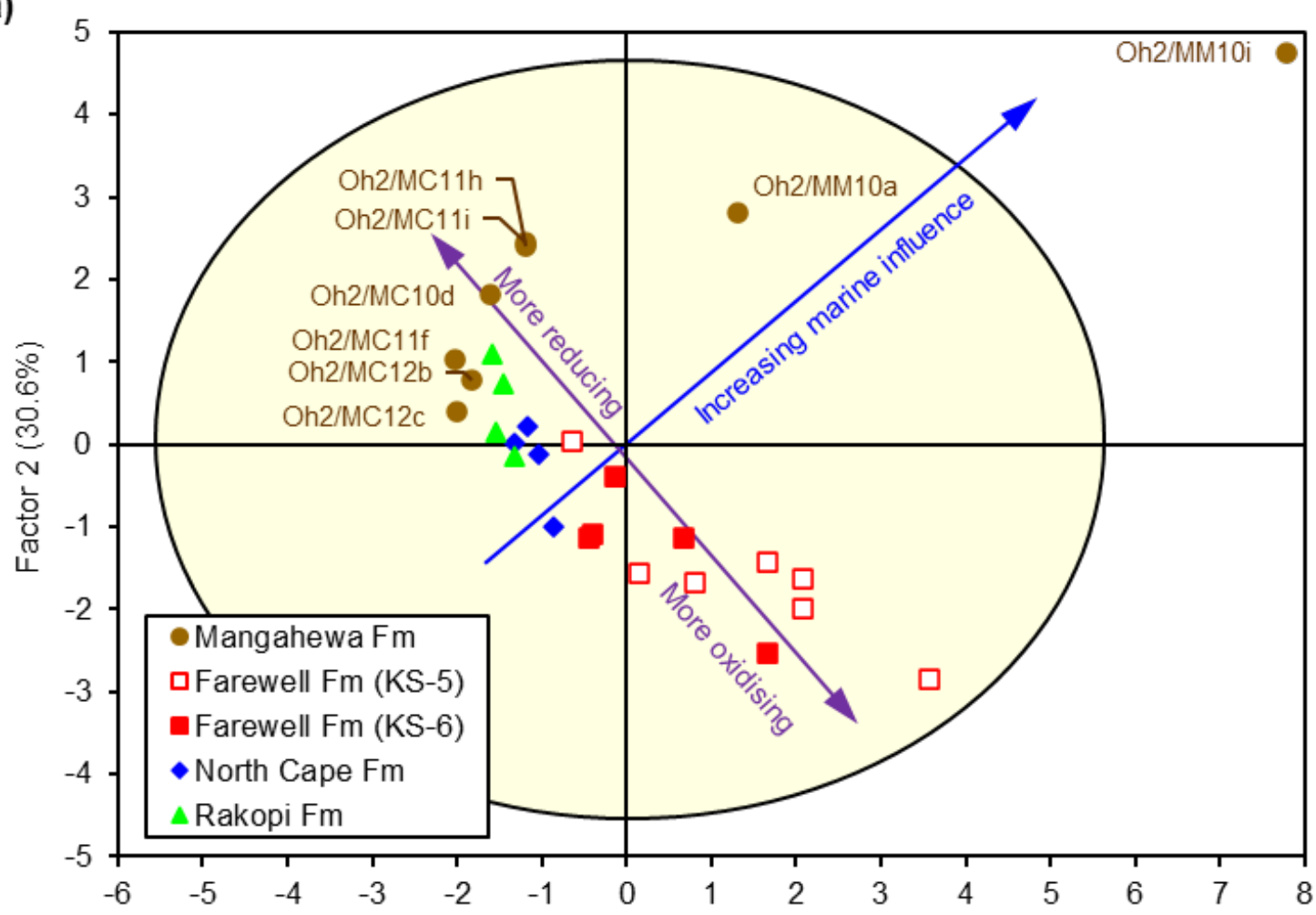

Factor $1(45.0 \%)$

b)

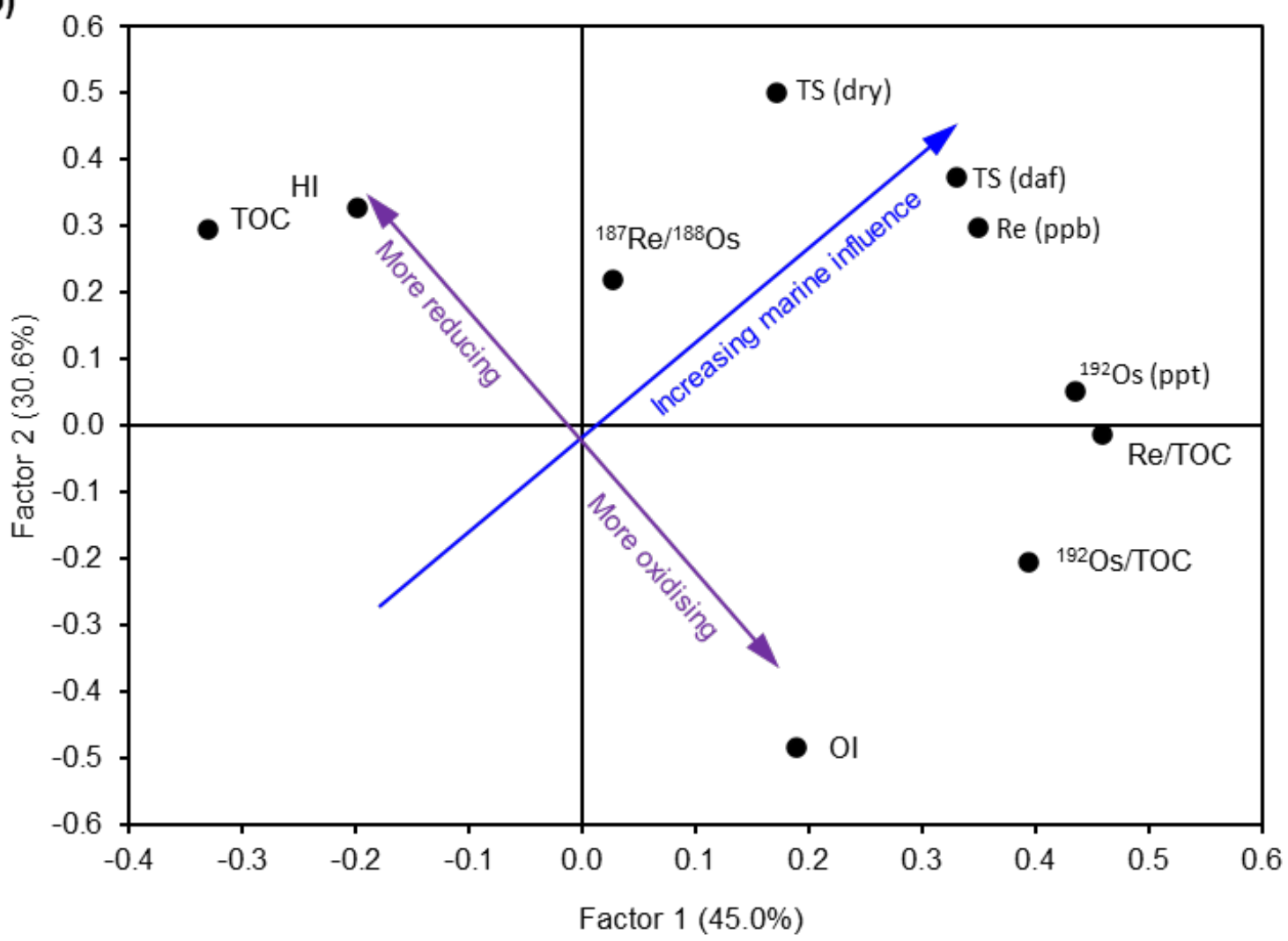

Fig. 4.8. a) Sample scores and b) parameter loadings on principal components 1 and 2 for the sample set. In a), the shaded ellipse represents $95 \%$ confidence limit. KS-5 and $-6=$ Kupe South-5 and -6 . 


\subsection{Discussion}

\subsubsection{Sources of Os in Taranaki coals and coaly mudstones}

The Osi values for sedimentary rocks reflect the ${ }^{187} \mathrm{Os} /{ }^{188} \mathrm{Os}$ of a water body at the time of deposition (Peucker-Ehrenbrink and Ravizza, 2000; Selby and Creaser, 2003; Cohen, 2004), which in turn results from the balance of Os sourced from two end-members: 1) rivers that drain older continental strata contributing to the radiogenic end-member $\left({ }^{187} \mathrm{Os} /{ }^{188} \mathrm{Os} \approx 1.4\right)$, and 2) magmatic activities, juvenile ocean crust and extra-terrestrial materials contributing to the non-radiogenic end-member $\left({ }^{187} \mathrm{Os} /{ }^{188} \mathrm{Os} \approx 0.12\right.$; Peucker-Ehrenbrink and Ravizza, 2000). The Osi values for samples from the Mangahewa, Farewell and North Cape formations are all moderately radiogenic, ranging between 0.31 and 0.51 (Fig. 4.9a). In contrast, samples from the Rakopi Formation are characterized by distinctly more radiogenic Osi values (0.81-1.24). Two possible explanations for the more radiogenic Osi of the Rakopi Formation compared to the younger formations are: 1) that seawater Os dominates for all formations but was more radiogenic in the late Cretaceous, or 2) the Os in this formation is dominated by Os from eroding continental strata rather than from seawater. Whilst the late Cretaceous seawater was more radiogenic than during deposition of the younger formations $(\sim 0.6$, compared with $\sim 0.3-$ 0.5), the Rakopi Formation Osi values are significantly more radiogenic (Fig. 4.9b). This suggests that deposition occurred in restricted mires that mainly source Os from weathering of the surrounding upper continental strata. This interpretation is consistent with paleogeographic reconstructions that indicate a mainly terrestrial depositional setting for the formation (Fig. 4.10). A slight marine influence is documented in the Rakopi coals, however, particularly in the uppermost interval where rare dinoflagellate cysts and glauconite grains are observed (Fig. 4.6f; Wizevich, 1994; King and Thrasher, 1996; Sykes et al., 2004; Browne et al., 2008). Sample RF/4 from this uppermost interval exhibits an Osi value (0.8) that is less radiogenic than those of the other samples from lower intervals of the formation (1.1-1.2; Fig. 4.9a). This suggests there may have been mixing of Os from the radiogenic continental run-off and the less radiogenic contemporaneous seawater in the uppermost interval. A similar mixing of terrestrial- and marine-sourced Os has been observed in modern macroalgae living in coastal (brackish) waters (Sproson et al., 2018).

The Osi values for samples from the Farewell and Mangahewa formations are very similar to ${ }^{187} \mathrm{Os} /{ }^{188} \mathrm{Os}$ of seawater at the time of their deposition ( $\sim 0.35$ and 0.50 , respectively; Fig. $4.9 \mathrm{~b}$ ), 
suggesting likely derivation of the Os from seawater. These Osi values also show very little variation over a wide range of Os concentrations (Fig. 4.9a), consistent with the samples deriving their Os signature from a single reservoir with well-mixed (homogeneous) ${ }^{187} \mathrm{Os} /{ }^{188} \mathrm{Os}$, such as the open ocean. This interpretation is further supported by palynological and geochemical data that show variable but significant marine influence in these samples (Section 4.5.3).

The Osi values (0.31-0.46) for the North Cape Formation sediments are slightly lower than the ${ }^{187} \mathrm{Os} /{ }^{188} \mathrm{Os}$ of seawater at the time of deposition ( $\sim 0.50 ; 67 \mathrm{Ma}$; Fig. 4.9$)$, and much lower than those of the Rakopi Formation sediments deposited within the same epoch (late Cretaceous). This indicates either a significant change in the nature of detrital sediment flux or localised changes in seawater ${ }^{187} \mathrm{Os} /{ }^{188} \mathrm{Os}$. The most likely source of less radiogenic Os is a large $(60 \mathrm{~km}$ across) subaerial volcanic centre named Vulcan/Hestia that erupted at the present-day Taranaki slope between 78 and $72 \mathrm{Ma}$ after the deposition of the Rakopi Formation, which they overlie (Fig. 4.2; Uruski, 2020). Seismic imaging of the geometry and stratigraphy of this volcanic centre shows a flat crest which has been interpreted to indicate that it was at least partially subaerial and eroding in the latest Cretaceous, sourcing sediments to the surrounding region for about 10 Myr (Uruski, 2020). The composition of the extrusives from this volcanic centre is currently unknown but is likely to be broadly similar to mafic rocks extruded at about the same time further north in the Deepwater Taranaki Basin (Romney volcano; Rad, 2015; Uruski, 2020). Weathering of such newly erupted mafic rocks has previously been shown to lower ${ }^{187} \mathrm{Os} /{ }^{188} \mathrm{Os}$ of sediments deposited in close vicinity, either directly as a sediment source or by lowering the Os composition of surrounding localised seawater (e.g., Rooney et al., 2016; Z. Liu et al., 2019b; Schröder-Adams et al., 2019). The presence of dinoflagellates, moderate to high total sulfur contents in the coals, and marine sedimentological structures such as mud drapes, burrows and bimodal ripples in sediments from the North Cape Formation suggest that brackish conditions were common throughout the deposition of this formation (Wizevich et al., 1992; King and Thrasher, 1996; Sykes and Dow, 2000). Therefore, it is likely that weathering of the mafic rocks lowered the ${ }^{187} \mathrm{Os} /{ }^{188} \mathrm{Os}$ of the series of complex waterways that characterised the southern part of the Taranaki Basin at $\sim 68 \mathrm{Ma}$ (Fig. 4.10c), which in turn influenced the deposition of the North Cape Formation. These waterways were likely to have been partially or fully restricted from the open ocean because the Osi values for the North Cape Formation samples are lower than that of global seawater at the time of deposition. 

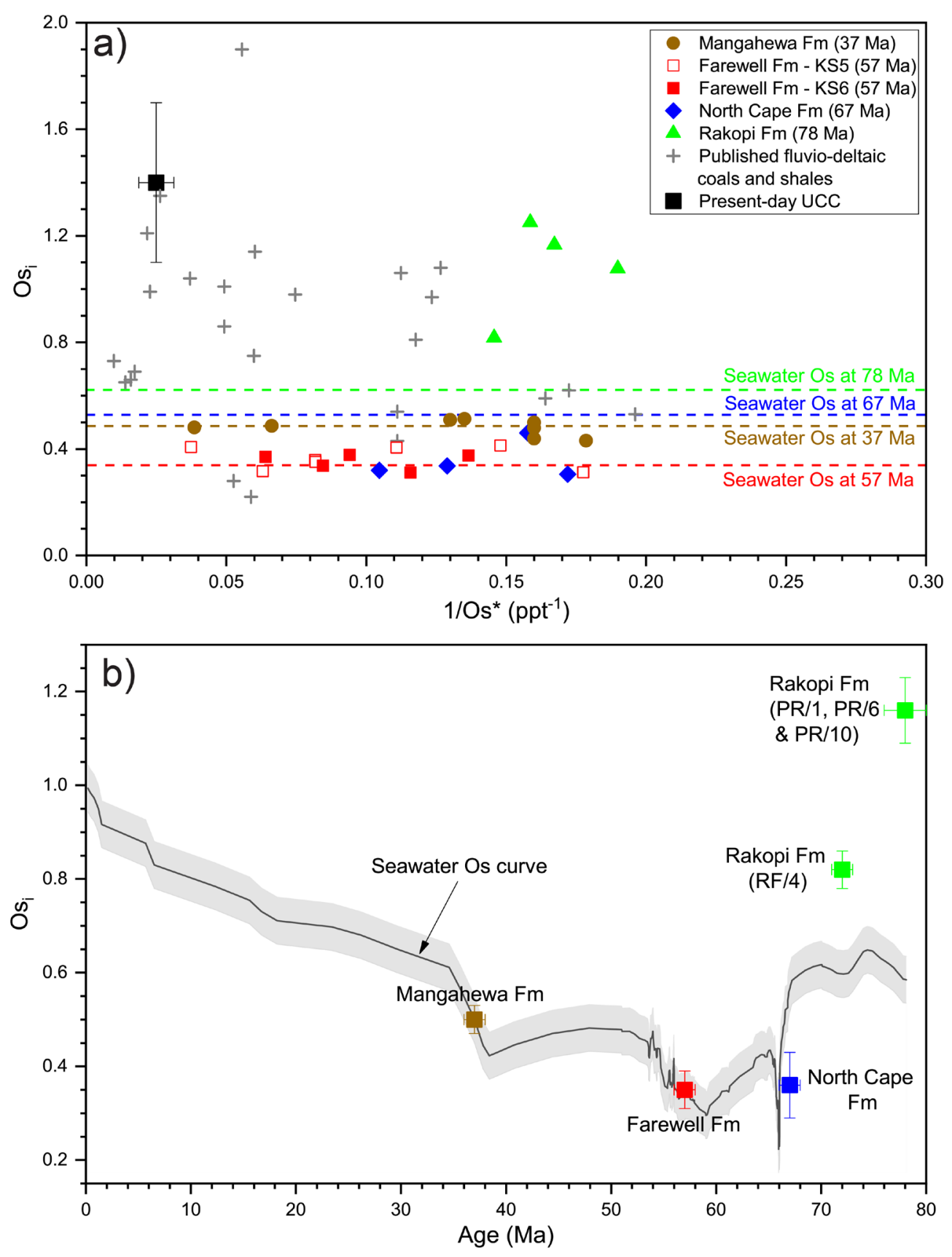

Fig. 4.9. a) A plot of initial ${ }^{187} \mathrm{Os} /{ }^{188} \mathrm{Os}\left(\mathrm{Os}_{\mathrm{i}}\right)$ versus $1 / \mathrm{Os}^{*}$ (reciprocal of common Os [Os corrected for radiogenic ingrowth of ${ }^{187} \mathrm{Os}$ ]) for samples from the Mangahewa, Farewell, North Cape and Rakopi formations. Published data for fluvio-deltaic coals and shales are included for comparison (Baioumy et al., 2011; Goswami et al., 2018). The seawater ${ }^{187} \mathrm{Os} /{ }^{188} \mathrm{Os}$ at the time of deposition of the four formations as well as that of the modern-day upper continental crust (UCC) are from PeuckerEhrenbrink and Ravizza (2012) and references therein. b) The Os $s_{i}$ for the Mangahewa, Farewell, North Cape and Rakopi formations plotted on the seawater Os curve for the past $80 \mathrm{Ma}$ (Peucker-Ehrenbrink and Ravizza, 2012). The shaded region represents a standard deviation of 0.1 in the Os curve. 
a) Mangahewa Formation

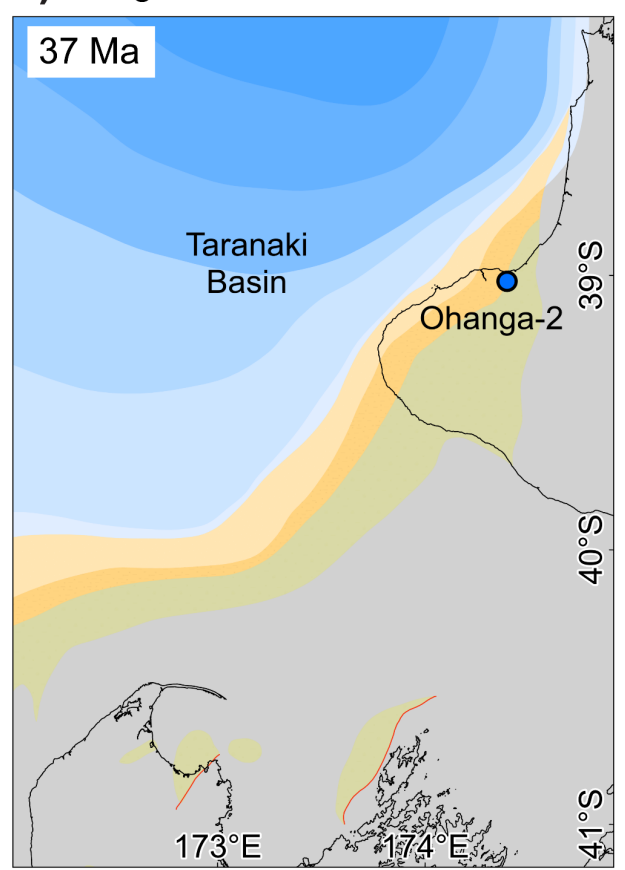

c) North Cape Formation

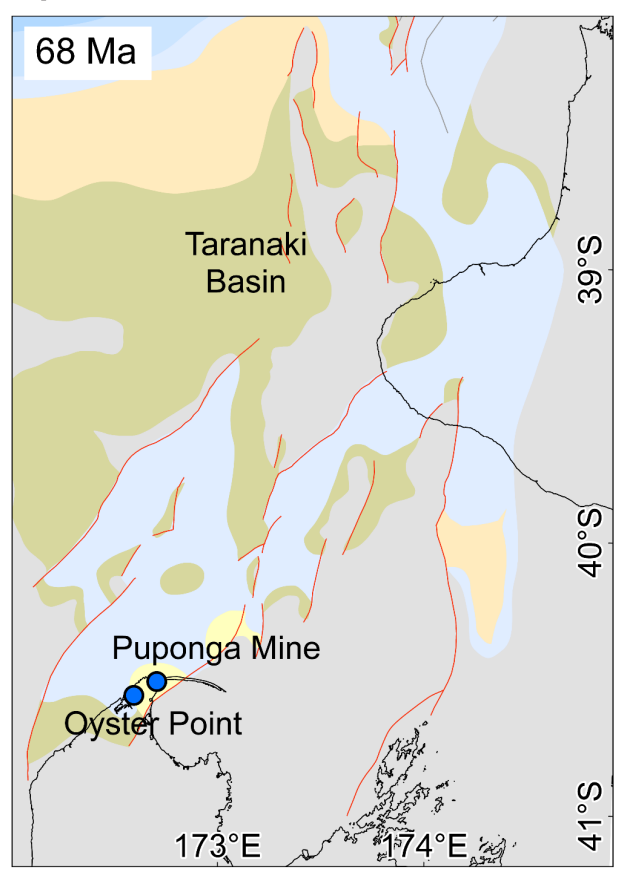

\section{b) Farewell Formation}

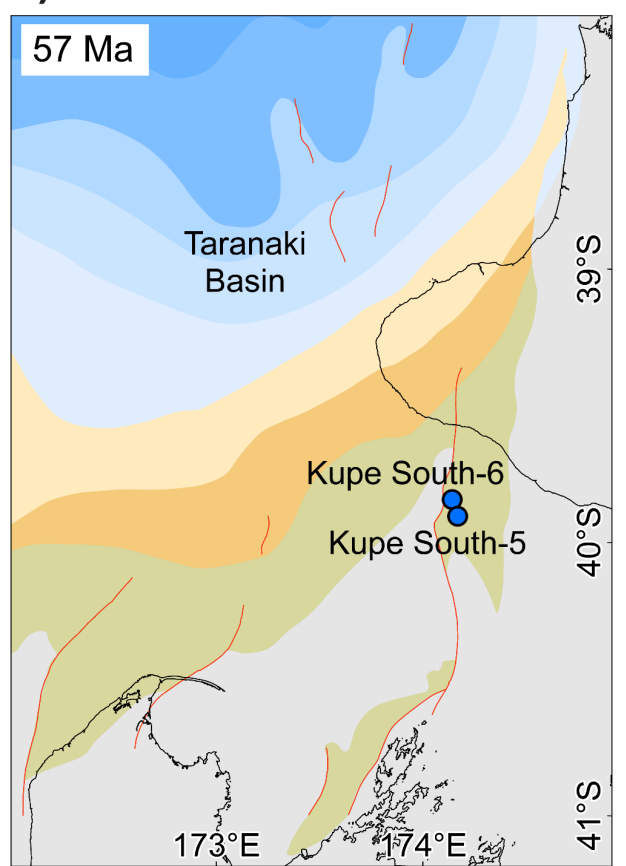

d) Rakopi Formation

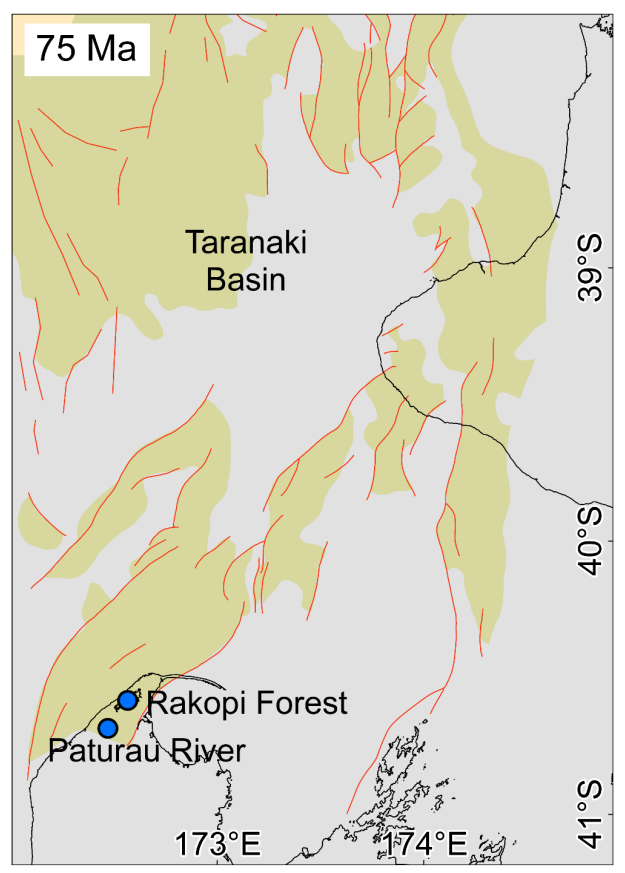

$\frac{d}{2}$

\begin{tabular}{|c|c|c|}
\hline Coastal plain, swamp, fluvial & Shoreface & Shelf \\
\hline Marginal marine & Alluvial fan & Slope \\
\hline Eroded/non-deposition & Present-day coastline & \\
\hline Active fault & Sample locations & \\
\hline
\end{tabular}

Fig. 4.10. Paleogeographic reconstructions of the Taranaki Basin during the deposition of the a) Mangahewa, b) Farewell, c) North Cape and d) Rakopi formations. Modified after Strogen (2011). 


\subsubsection{Effects of coal petrofacies on Re-Os systematics}

There are two main types of coal seam facies represented amongst New Zealand humic coaly rocks: 1) planar mire coals and coaly mudstones, and (2) raised mire coals (Sykes et al., 2014). Planar mire coals are thin and were formed from peats that accumulated under relatively high rates of accommodation space increase in a syn-rift setting. Groundwater levels within these mires were consistently high and the vegetation was well fed via nutrients within the underlying mineral substrate and frequent inundation of clastics during flooding (Edbrooke et al., 1994; Sykes et al., 2004). These mires are therefore rheotrophic (flow-fed) or eutrophic (nutrientrich). The Rakopi, North Cape and Farewell coals and coaly mudstones are all planar mire facies.

In contrast, raised mire coals, which are represented by the Mangahewa Formation coals in this study, formed in a passive margin setting under more moderate rates of accommodation space increase and under an ever-wet climate that allowed for the development of thick coal seams (Edbrooke et al., 1994; Sykes et al., 2004). The upper surface of raised mire coals is normally above river flood levels and vegetation roots no longer have access to the underlying mineral substrate. The only nutrients are therefore from rainwater. These peat mires are thus oligotrophic (nutrient-poor) and ombrotrophic(rain-fed), and the resulting coal facies can be very low in trace element concentrations due to the leaching that takes place when rainwater drains down through the peats.

These differences in coal petrofacies might, therefore, explain the lower Re concentrations in four Mangahewa Formation coals compared with the majority of the Rakopi, North Cape and Farewell coals (Fig. 4.7a). The likely leaching of elements in ombrotrophic coals may also partly explain why the Mangahewa Formation coals are low in Re and Os, while the Mangahewa Formation coaly mudstones have higher concentrations of these elements. An alternative explanation for this is that the Mangahewa Formation coaly mudstones are from the base and roof of the seam 10, where marine influence is greatest (Sykes et al., 1999; Section 4.5.3). 


\subsubsection{Effects of marine influence on Re-Os systematics of fluvio-deltaic sediments}

Total sulfur content is a sensitive indicator of marine influence in fluvio-deltaic sediments, with coals having high TS content commonly occurring immediately below marine sediments (Williams and Keith, 1963; Banerjee and Goodarzi, 1990; Diessel, 1992; Gayer et al., 1999; Sykes, 2001; Chou, 2012; Sykes et al., 2014). The high TS content in these coals originates from bacterial reduction of seawater sulfate to elemental $\mathrm{S}, \mathrm{H}_{2} \mathrm{~S}$ and polysulfides, which then react with ferrous iron and organic matter to produce pyrite and organic S compounds, respectively (Calkins, 1994; Chou, 2012; Zheng et al., 2014). Based on extensive analyses of New Zealand coals, $0.5 \%$ TS (daf) is taken as the approximate upper limit for coals deposited in entirely terrestrial environments (e.g., Suggate, 1959; Edbrooke et al., 1994; Sykes et al., 1999; 2014; Sykes, 2001; 2004; Browne et al., 2008; Higgs et al., 2012). Accordingly, samples exhibiting $0.5-1.5 \%$ (daf) TS content are classified as slightly marine-influenced and those with TS content $>1.5 \%$ (daf) are classified as strongly marine-influenced (Sykes, 2001; 2004; Sykes et al., 2014). In this study, I also adopt these classifications and consider all samples with TS content above $0.5 \%$ (daf) to indicate at least some degree of marine influence (Fig. 4.6f).

The TS content for samples from the Rakopi, North Cape and Farewell formations range from 0.2 to $1.5 \%$ (daf), indicating entirely terrestrial to moderately marine-influenced depositional conditions. Marine influence has been suggested to cause significant enrichment of Re and Os in coals (up to $\sim 2$ orders of magnitude higher than freshwater coals; Tripathy et al., 2015). However, none of the samples from the three formations are significantly enriched in Re and Os, with concentrations levels that are comparable to those of fully terrestrial fluvio-deltaic coals and coaly mudstones (Baioumy et al., 2011; Goswami et al., 2018). In the Farewell Formation samples from Kupe South-5 core, Os concentrations increase stratigraphically upwards (except sample KS5/1b), reaching the highest concentration (46 ppt) in sample KS5/1a, which contains rare dinoflagellate cysts (Table 4.1; Fig. 4.4a; Morgans and Pocknall, 1991; Flores et al., 1998). This may indicate a progressive increase in the degree of brackishness of the mire and subsequently, the amount of Os derived from the seawater. However, this increase in Os concentrations is not commensurate with variations in Re concentrations and shows no relationship with TS (Table 4.1). Therefore, the observed trend in Os content is unlikely to have directly resulted from the increasing marine influence in these 
samples. There are two likely explanations for the lack of significant Re and Os enrichment in samples from the Rakopi, North Cape and Farewell formations: 1) the documented marine influence may have been too slight or too late (post-depositional) to enable substantial sequestration of these elements from the seawater or 2) the prevailing depositional conditions were not suitable for chelation of the elements to the organic matter.

The Mangahewa Formation samples offer an opportunity to evaluate the impact of strong synsedimentary marine influence on Re-Os systematics of fluvio-deltaic sediments. In these samples, TS content range from 0.9 to $62.6 \%$ (daf), indicating slight to very strong marine influence (Sykes et al., 1999). This is consistent with common occurrences of dinoflagellate cysts, Ophiomorpha burrows and mangrove pollen in these samples, and interpretations of a tidally-influenced coastal plain to marginal marine depositional setting (Figs 5a, 10a; Sykes et al., 1999; Flores, 2004; Higgs et al., 2006; Sykes et al., 2014). Other conspicuous signs of marine influence within the Mangahewa coals include diminished vitrinite reflectance, enhanced vitrinite fluorescence and stratigraphic correlations between HI and TS content (Sykes et al., 1999; 2014). The marine influence within the Mangahewa coals has been shown to be syn-sedimentary based on moderately strong, positive correlations between TS and aromatic compounds of sulfur, such as dibenzothiophene (Volk et al., 2008). Despite the strong syn-sedimentary marine influence, none of the Mangahewa Formation samples is significantly enriched in Re and Os, with concentrations that range from 0.11 to $1.33 \mathrm{ppb}$ and 14.2 to 66.2 ppt, respectively (Fig. 4.7). Strong positive correlations between TS content and Re and Os concentrations in these samples suggest probable derivation of the elements from the seawater. However, these concentrations are up to two orders of magnitude lower than the average Re (108 ppb) and Os (520 ppt) concentrations in the marine-influenced Matewan coals, despite apparent comparable levels of marine influence (Geboy et al., 2015; Tripathy et al., 2015). This suggests that depositional conditions necessary for chelation of Re and Os in the Mangahewa Formation coals and coaly mudstones may have been limited.

The factors controlling Re and Os chelation into organic-rich sedimentary rocks are currently not well understood, making it difficult to establish exact conditions that may have limited Re and Os uptake in the Mangahewa Formation samples. However, it is unlikely to relate to redox conditions of the water column (e.g., Crusius et al., 1996; Crusius and Thomson, 2000; Yamashita et al., 2007) because the average OI values are very low in both the Matewan (2 mg 
$\mathrm{CO}_{2} / \mathrm{g}$ TOC; Tripathy et al., 2015) and Mangahewa samples (4 $\mathrm{mg} \mathrm{CO}_{2} / \mathrm{g}$ TOC), indicating similar oxygen-poor depositional conditions; possibly sulfidic due to the high sulfur content and abundant pyrite in both sets of samples. It is also unlikely that organic matter type (Type II/III; Fig. 4.6c, d; Rooney et al., 2012) and maceral composition (vitrinite, inertinite and liptinite $=85.5,8.4$ and 6.1 vol. $\%$, mineral-matter-free [mmf], respectively) of the Mangahewa Formation samples limited the uptake of Re and Os because they are comparable to those of the Matewan coals (Type II/III and vitrinite, inertinite and liptinite composition of 79.1, 14.7 and 6.2 vol.\%, mmf, respectively; Geboy et al., 2015; Tripathy et al., 2015), and thus may have similar Re and Os organic-chelating capabilities. Basin restriction and low seawater renewal frequency are other factors that may cause inefficient replenishment and subsequent depletion of Re and Os in peat-forming mires (Turgeon et al., 2007; McArthur et al., 2008). However, these factors are unlikely to have caused the low Re and Os concentrations in the Mangahewa coals because the Osi values $(0.43-0.51 ; \overline{\mathrm{x}}=0.48)$ for these samples overlap that of the seawater at the time of deposition ( $\sim 0.5$; Fig. $4.10 \mathrm{~b})$. Such a similarity suggests that either (1) the Mangahewa paleomire was interconnected to the global ocean or (2) the isotopic composition of Os flux into the mire from the weathering of the surrounding continental mass was extremely similar. The latter is highly unlikely because there are no major late Eocene igneous extrusions in the Taranaki Basin that could lower this value and the radiogenic samples from the Rakopi Formation (mean Osi value of 1.1) imply that the New Zealand continental mass must have been contributing radiogenic Os flux.

The key difference between the Matewan and Mangehewa coals, and indeed the other coal formations that have been analysed for Re and Os (Baioumy et al., 2011; Goswami et al., 2018), is that the Matewan coalbed is directly overlain by a marine shale (Betsie Shale Member; Geboy et al., 2015; Tripathy et al., 2015). This may have allowed Re- and Os-rich seawater to percolate down and be fixed by reduction into the Matewan coals. Seams 10 and 11 of the Mangahewa coals show increases in total and pyritic sulfur towards the top and bottom of seams, and these trends finish with the presence of dinoflagellate cysts and mangrove pollen in the roof and floor sediments, as well as an Ophiomorpha burrow in the roof of seam 10 (Sykes et al., 1999). These trends indicate marginal marine, brackish conditions, but not fully marine conditions and thus, represent lowstand deposits at the turnaround of short regressivetransgressive cycles within an overall transgressive regime. A similar upward decreasing then increasing trend of sulfur content is seen across the Rakopi coal seams in the Paturau River 
section (Sykes et al., 2004), which again indicates a change in shoreline retreat to advance, but in this case at a far greater distance from the actual shoreline. With very low gradient coastal plains, freshwater-brackish water interfaces can extend several tens of kilometres inland from the coast, to impart elevated sulfur contents in peat mires (Bohacs and Suter, 1997; Sykes et al., 2014). However, these are not fully marine conditions which may explain limited enrichment and homogenisation of Re and Os in the Rakopi and Mangahewa coals. Such enrichment and homogenisation of Re and Os in coals might occur only in the rare situation where coal seams are directly overlain by truly marine organic-rich shales, such as is the case for the Matewan coals.

\subsubsection{Re-Os geochronology in fluvio-deltaic coals and coaly mudstones}

The Farewell Formation samples from Kupe South-5 and -6 were analysed with the aim of constraining their Re-Os depositional age. Regression of the ${ }^{187} \mathrm{Re} /{ }^{188} \mathrm{Os}$ and ${ }^{187} \mathrm{Os} /{ }^{188} \mathrm{Os}$ ratios for these samples, however, shows significant scatter with no discernible linear trend (Fig. 4.11). The only previous attempt at producing Re-Os depositional ages for fluvio-deltaic sediments also reported large scatter in ${ }^{187} \mathrm{Re} /{ }^{188} \mathrm{Os}$ and ${ }^{187} \mathrm{Os} /{ }^{188} \mathrm{Os}$ ratios for entirely nonmarine coals and shales from the Soldogg Formation, Finnmark Platform (Goswami et al., 2018). The lack of an isochron fit in the Soldogg Formation sediments was attributed to heterogeneity in the Osi, which varied by 0.5 units. However, unlike the Soldogg Formation sediments, samples from the Farewell Formation exhibit Osi values that vary by only 0.1 units (Table 4.1). Published Re-Os data for several marine and terrestrial sample suites also show more variability in the Osi values than both the Farewell and Soldogg Formation samples, but still yield reliable Re-Os depositional ages. For example, comparably aged Douglas Creek Member of the lacustrine Green River Formation, USA, exhibits Osi values that vary by $\sim 0.2$ units but still produce an accurate, albeit imprecise, depositional age of $49.8 \pm 4.8 \mathrm{Ma}$ (Cumming et al., 2012). Similarly, the marine-influenced Matewan Coal bed, USA, and Kaimur shales, India, yielded accurate but imprecise Re-Os depositional ages (325 $\pm 14 \mathrm{Ma}$ and $1210 \pm 52 \mathrm{Ma}$, respectively) despite Osi values that varied by 0.7 and 1.1 units, respectively (Tripathy et al., 2015; Tripathy and Singh, 2015). The key feature that allowed the Douglas Creek Member shales, Matewan coals and Kaimur shales to develop reliable isochrons despite significant variation in Osi ratios appears to relate mainly to large variations in the ${ }^{187} \mathrm{Re} /{ }^{188} \mathrm{Os}$ ratios (ranges of $\sim 1500,2400$ and 850 units, respectively) compared to those in the Soldogg 
and Farewell Formation samples (93 and 145 units, respectively). However, sample suites with similarly limited variations in ${ }^{187} \mathrm{Re} /{ }^{188}$ Os have developed reliable isochrons (e.g., Selby et al., 2009; Rooney et al., 2014; Sperling et al., 2014). For instance, the Aptian-Albian boundary interval in northwest Germany exhibits ${ }^{187} \mathrm{Re} /{ }^{188}$ Os values that vary by only $\sim 70$ units, but yields a Re-Os age $(108.9 \pm 6.2 \mathrm{Ma})$ that is within uncertainty of the Geological Time Scale 2016 determination (Selby et al., 2009). In this interval, however, the Osi values vary by only 0.002 units, thereby permitting an isochron fit despite the low spread in ${ }^{187} \mathrm{Re} /{ }^{188} \mathrm{Os}$ ratios. Therefore, either very low Osi variability or large ranges in ${ }^{187} \mathrm{Re} /{ }^{188} \mathrm{Os}$ values is required, but not necessarily both. The Farewell Formation samples lack both features, hindering the development of an isochron.

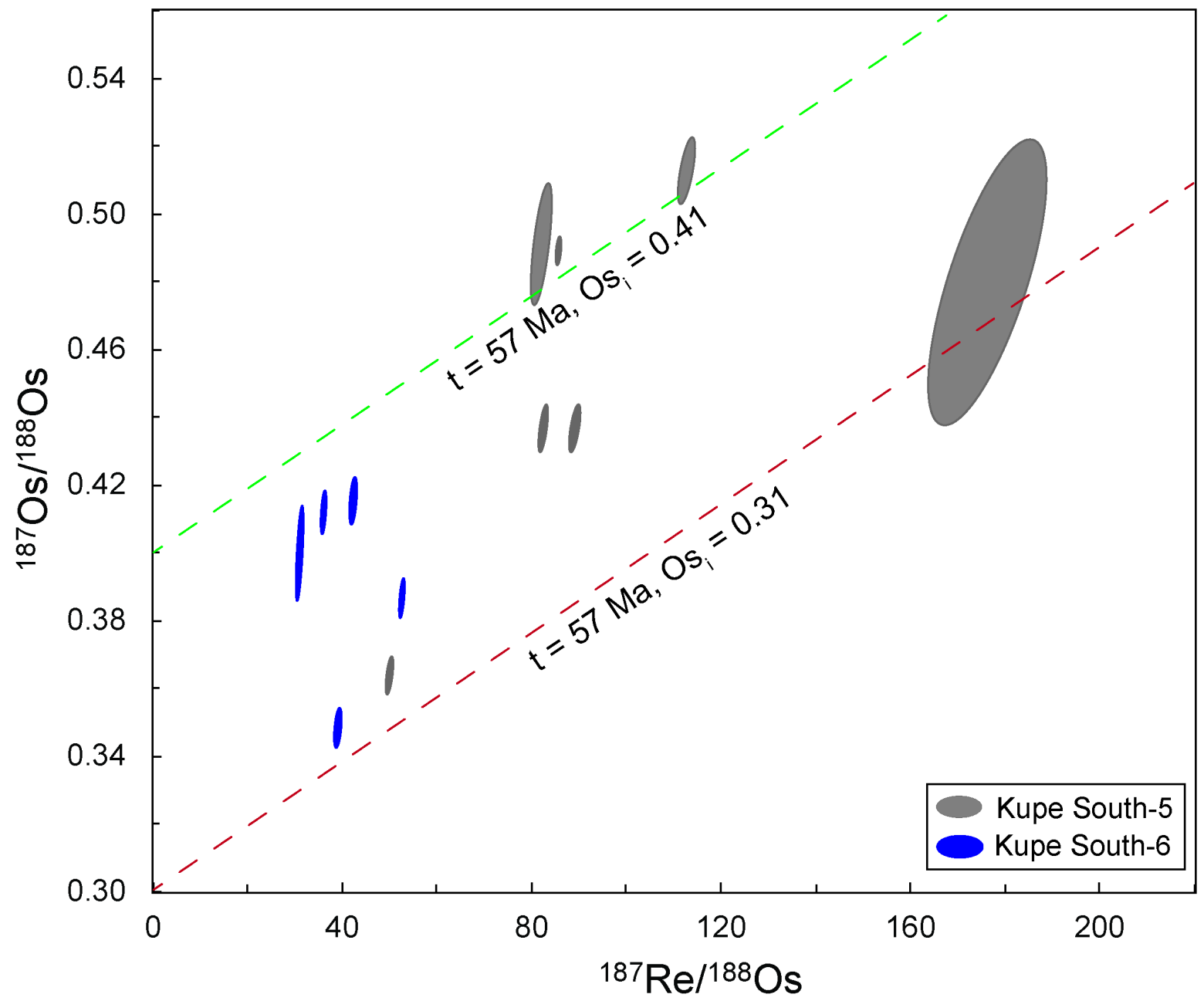

Fig. 4.11. Re-Os isochron diagram for the Farewell samples from Kupe South-5 and -6. There is a large scatter in the data without any noticeable linear trend. Reference lines with slopes equivalent to the approximate age of the samples (57 Ma; Crouch and Raine, 2012) and $\mathrm{Os}_{\mathrm{i}}$ values of 0.31 and 0.41 (minimum and maximum values) are shown by the red and green dashed lines, respectively. 
Fluvio-deltaic sediments appear to rarely attain the levels of Osi homogeneity commonly seen in marine shales. There are two likely explanations, which are not mutually exclusive: 1) the source materials for these sediments (detrital silicates and plant debris) are typically heterogeneous, or 2) deposition occurs in restricted basins that prevent complete mixing and homogenisation of the Os signature before sequestration into the sedimentary record. Of the organic-rich sample suites studied here, the coals and coaly mudstones of the Mangahewa Formation are the most marine-influenced (Section 4.5.3; Sykes et al., 1999). The Osi values for these samples are also identical to that of seawater at the time of deposition, indicating that the Mangahewa paleomire and the open ocean were interconnected, allowing for homogenisation of the Os signature (Tripathy et al., 2015). It is, therefore, unsurprising that six of the eight samples from this formation exhibit Osi values that vary by only 0.03 units (Table 4.2) and appear to form a linear trend on an isochron diagram (Fig. 4.12a). The produced isochron age of $28 \pm 16 \mathrm{Ma}$ (Mean Square of Weighted Deviates [MSWD] $=1.5 ; \mathrm{Os}_{\mathrm{i}}=0.50 \pm$ 0.03 ) is, however, imprecise and younger than, albeit within uncertainty of, the estimated biostratigraphic age (37 Ma) for the samples (Fig. 4.12a). A $37 \mathrm{Ma}$ reference line with an assumed Osi value of 0.48 passes through all the data points (Fig. 4.12a), implying that the large uncertainties in the measured Re-Os data for these samples are the main reason for the imprecise Re-Os age. A Monte Carlo simulation of the uncertainties (Fig. 4.12b; Li et al., 2019) shows that $69 \%$ of the total uncertainties in the isochron age come from analytical uncertainties, which likely resulted from the low Re and Os concentrations in the Mangahewa Formation samples and small amounts of rock powder $(0.2 \mathrm{~g})$ used for analysis (Section 4.3.2). The samples also represent a wider stratigraphic interval $(\sim 5 \mathrm{~m})$ which may have introduced variations in Osi values. As with the Farewell Formation samples, a lack of spread in ${ }^{187} \mathrm{Re} /{ }^{188} \mathrm{Os}$ ratios (97 units) is also a feature of the Mangahewa Formation samples that may have further contributed to the imprecision of the Re-Os age. This low spread in ${ }^{187} \mathrm{Re} /{ }^{188} \mathrm{Os}$ ratios is in stark contrast to that of the similarly marine-influenced Matewan coal ( 1800 units), further highlighting the importance of understanding the factors that may have controlled Re and Os uptake and fractionations in the two formations if sampling for geochronology purposes. 

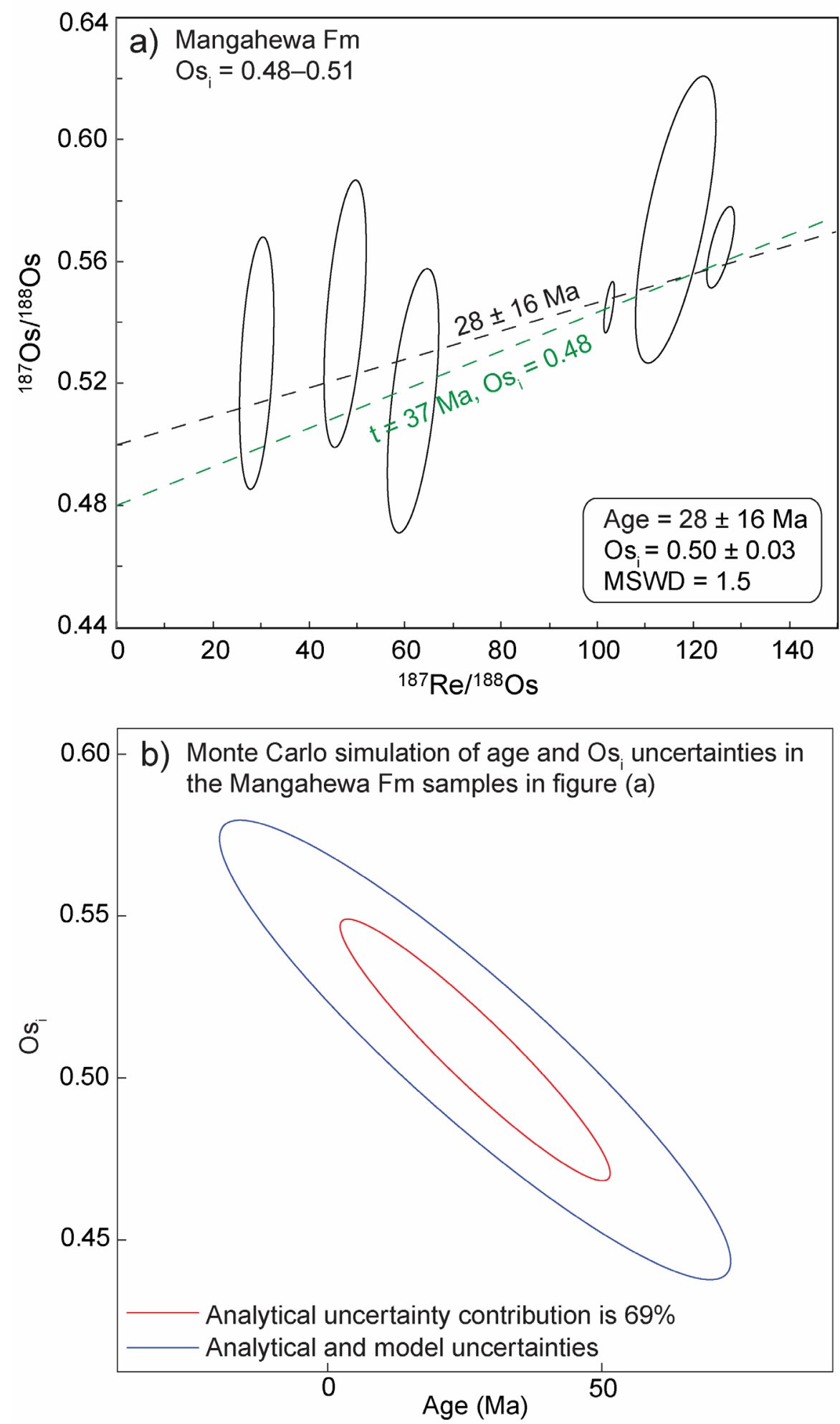

Fig. 4.12. a) Re-Os isochron diagram for Mangahewa samples with $\mathrm{Os}_{\mathrm{i}}$ values between 0.48 and 0.51 (six of the eight samples analysed). The data appear to form an isochron (black dashed line), but the age produced is imprecise and inaccurate $(28 \pm 16 \mathrm{Ma})$. A reference line with a slope equivalent to the biostratigraphic age of the samples (37 Ma) and an $\mathrm{Os}_{\mathrm{i}}$ value of 0.48 passes through all the data points (green dashed line). b) Monte Carlo simulation of uncertainties in the Os $\mathrm{s}_{\mathrm{i}}$ value and age of the six Mangahewa samples in Figure 4.12a (Li et al., 2019), showing that $69 \%$ of the total uncertainties are derived from analytical uncertainties. See text for further discussion. 
Many instances of low spread in ${ }^{187} \mathrm{Re} /{ }^{188} \mathrm{Os}$ ratios have been reported for both marine and terrestrial sediments (e.g., Turgeon et al., 2007; Selby et al., 2009; Zhu et al., 2013; Rooney et al., 2014; Sperling et al., 2014; Liu et al., 2018). The Re-Os isotope data for the Farewell Formation samples from Kupe South-5 and -6 cores support previous findings that the stability and variability of the depositional environment exert some control on Re-Os fractionation (Cumming et al., 2012; Harris et al., 2013; Liu et al., 2020). The Kupe South-5 samples exhibit six times larger spread in ${ }^{187} \mathrm{Re} /{ }^{188}$ Os ratios (126 units) than those from the Kupe South-6 (21 units), potentially due to the difference in their depositional conditions. The Kupe South-5 samples are interpreted as paleosols, deposited in low-lying swamps that were proximal to an active channel (Flores et al., 1998) and thus, generally susceptible to fluctuating high and low energy clastic sedimentation during fluvial floods. These samples contain abundant but variable vitrain (wood) lenses and streaks, leaves and macerated plant fragments (Flores et al., 1998) which may have provided variable chelating surfaces for complexation of Re and Os, leading to increased ${ }^{187} \mathrm{Re} /{ }^{188}$ Os fractionation (Cumming et al., 2012). In contrast, the sampled interval in Kupe South-6 lacks coarse sediments and paleosols, and exhibits a thickening upward linsen lamination that is characteristic of sediments deposited in a distal floodplain lake setting (Constantine, 2008). This more stable depositional setting may have allowed a more homogeneous uptake of Re and Os leading to the lower spread in ${ }^{187} \mathrm{Re} /{ }^{188} \mathrm{Os}$ ratios of the Kupe South-6 samples, consistent with the findings of Cumming et al. (2012).

\subsection{Conclusions}

This study presents Re-Os, S and bulk pyrolysis data for coals and coaly mudstones from the Taranaki Basin, New Zealand, to investigate factors controlling Re-Os systematics and geochronology in fluvio-deltaic sediments. Samples from the late Cretaceous Rakopi Formation exhibit radiogenic Osi values ranging from 0.8 to 1.2 , expected for fully terrestrial coals deposited in restricted mires, and which mainly source Os from the weathering of surrounding older continental strata. In contrast, samples from the progressively younger North Cape, Farewell and Mangahewa formations record non-radiogenic Osi values (0.3-0.5), which is attributed to variable proportions of Os derived from seawater (Farewell and Mangehewa) and contributions from nearby freshly erupted mafic extrusives (North Cape). 
The Re (0.11-1.33 ppb) and ${ }^{192} \mathrm{Os}(5.6-29.5 \mathrm{ppt})$ concentrations for the strongly marineinfluenced coals from the Eocene Mangahewa Formation are very low in comparison with similarly marine-influenced coals from the US Matewan coal bed. The Matewan coal bed is directly overlain by a marine shale, suggesting that such a juxtaposition of depositional environments may be required for enhanced Re and Os enrichment, for example through postdepositional percolation of $\mathrm{Re}$ and Os-rich seawater from the overlying marine shale. Therefore, Re and Os enrichment in coals does not simply correlate with the level of marine influence, the timing and nature of the marine influence as well as the presence of conditions necessary for chelation of the elements are equally important. Despite the low Re and Os concentrations, the strongly marine-influenced Mangahewa Formation samples exhibit Os isotopic compositions that are better equilibrated compared to those of the Farewell Formation with a slight marine influence. These samples develop a linear trend on an isochron diagram, producing an age that, although imprecise, is within uncertainty of the samples' biostratigraphic age. In contrast, the ${ }^{187} \mathrm{Re} /{ }^{188} \mathrm{Os}$ and ${ }^{187} \mathrm{Os} /{ }^{188} \mathrm{Os}$ of the slightly marineinfluenced samples from the Farewell Formation display significant scatter without any linear relationship.

The factors controlling Re-Os fractionation in organic-rich sediments were evaluated for Farewell Formation samples from the Kupe South-5 and -6 cores. The Kupe South-5 samples exhibit six times more spread in ${ }^{187} \mathrm{Re} /{ }^{188}$ Os ratios (126 units) than those from the Kupe South6 (21 units), which is attributed to differences in stability and variability of the depositional environments of the two sample sets. The Kupe South-5 samples were deposited in low-lying swamps that were proximal to an active channel with fluctuating high and low energy clastic sedimentation. In addition, these samples comprise variable vitrain (wood) lenses and streaks, leaves and macerated plant fragments that may have provided variable chelating surfaces for complexation of Re and Os, leading to higher ${ }^{187} \mathrm{Re} /{ }^{188} \mathrm{Os}$ fractionation. In contrast, samples from the Kupe South-6 lack coarse sediments and paleosols, and were deposited in a distal, more stable floodplain lake setting. Such a stable depositional setting has previously been suggested to allow a more homogeneous uptake of Re and Os, leading to the lower spread in ${ }^{187} \mathrm{Re} /{ }^{188}$ Os ratios. 


\section{RE-OS SYSTEMATICS AND OS ISOTOPE FINGERPRINTING IN CRUDE OILS SOURCED FROM TERRESTRIAL COALS AND MARINE SHALES, NEW ZEALAND}

\section{Preface}

A version of this chapter will be submitted into Journal of Petroleum Geology, co-authored by Monica Handler (MH), Richard Sykes (RS), David Selby (DS) and Sebastian Naeher (SN).

Most of the samples were provided by RS from a library of oils held at GNS Science, Lower Hutt. Additional samples were obtained by myself and SN from the National Core Store in Featherston. Sample preparation, laboratory analyses, data interpretation and manuscript preparation were undertaken by myself with suggestions and comments from all co-authors. MH, SN, DS and technicians at Durham University, Victoria University of Wellington and GNS Science assisted in setting up laboratory protocols and training on Re-Os mass spectrometry. Sulfur analyses were undertaken by CRL Energy Ltd. Complementary biomarker data were available from PBE online data portal, as part of New Zealand oils and oil show databases. 


\subsection{Introduction}

The Re-Os isotope system has shown great potential for dating and tracing crude oils to their source in marine and lacustrine petroleum systems (Selby et al., 2005a; 2007; Selby and Creaser, 2005a; Finlay et al., 2011; 2012; Lillis and Selby, 2013; Cumming et al., 2014; Georgiev et al., 2016; 2019; Ge et al., 2018; Liu et al., 2018; Corrick et al., 2019; Scarlett et al., 2019; Hurtig et al., 2020). These studies show that Re-Os geochronology of crude oils can yield isochron ages that match the timing of oil generation, as confirmed independently by other techniques such as basin modelling and ${ }^{40} \mathrm{Ar} /{ }^{39} \mathrm{Ar}$ dating of fluid inclusions. In addition, the ${ }^{187} \mathrm{Os} /{ }^{188} \mathrm{Os}$ of crude oil has been shown in both hydrous pyrolysis experiments and field studies to reflect that of the source rocks at the time of oil generation, providing a powerful inorganic geochemical tracer for correlating the oils to their source (Selby et al., 2005a; Finlay et al., 2011; Rooney et al., 2012; Cumming et al., 2014; Liu et al., 2018). The potential of the Re-Os isotope system to date and provide insights into oil-source correlation in coaly-sourced petroleum systems has, however, not been explored. Recent studies show that the Re-Os isotope system may not provide meaningful geochronological information for terrestrial coals due to the heterogeneity of initial ${ }^{187} \mathrm{Os} /{ }^{188} \mathrm{Os}$ ratios and the similarity in ${ }^{187} \mathrm{Re} /{ }^{188} \mathrm{Os}$ ratios (Goswami et al., 2018; Chapter 3 of this thesis). However, the processes that lead to Os isotope homogenisation in marine and lacustrine crude oils, such as primary migration (Liu et al., 2018), may also apply to coaly-sourced oils. In addition, Os isotopes may offer valuable source information for these oils, provided estimates of oil generation dates are available through other means such as basin modelling.

This study explores the applicability of the Re-Os isotopic system to crude oils sourced from late Cretaceous coaly source rocks of the Rakopi and North Cape formations, Taranaki Basin, New Zealand (Sykes et al., 2012). These source rocks have previously been characterised for their Re-Os isotopic compositions in Chapter 4 of this thesis, allowing for an assessment of the suitability of the Os isotope fingerprinting tool to trace coaly-sourced oils. In addition, this study explores the behaviour of Re and Os in a family of distinctive marine oils from the Kora1 well in the northern part of the Taranaki Basin. These oils have unusual organic geochemical signatures which include: a high proportion of terrestrial organic matter as indicated by abundant terrestrial plant biomarkers, heavy carbon isotope $\left(\delta^{13} \mathrm{C}\right)$ signatures of total saturate $(>-26 \%)$ and aromatic $(>-23 \%)$ hydrocarbon fractions, and exceptionally high $\mathrm{C}_{30}$ sterane 
content. These properties correlate to those of the late Paleocene Waipawa Formation (Sykes et al., 2012; Naeher et al., 2019), which has also been characterised for its Re-Os isotope composition (Chapter 3). Lastly, this study investigates the utility of the Os isotope fingerprinting technique in constraining the source of two marine oils that seep out on the northern and southern parts of New Zealand's East Coast Basin whose origin remains enigmatic (Rogers et al., 1999; Sykes et al., 2012).

\subsection{Geological setting}

New Zealand oils are grouped into five genetic oil tribes and 11 families, which are primarily represented in the Taranaki Basin but with members found in other sedimentary basins (Fig. 5.1; Sykes et al., 2012). Four of these tribes are sourced from terrestrial, coal-bearing sequences and are mainly distinguished by variations in the degree of marine influence, anoxicity and relative inputs of angiosperms and gymnosperms to the source kerogen (Sykes et al., 2012). Within the Taranaki Basin, the genetic oil families tend to be geographically constrained, which has been useful in identifying likely source kitchens and migration pathways (Sykes et al., 2012). This study investigates oil accumulations genetically grouped under oil families 11, 41 (Taranaki Basin) and 42 (East Coast Basin; Fig. 5.1).

\subsubsection{Taranaki petroleum system}

The Taranaki Basin is the only hydrocarbon producing basin in New Zealand, with estimated recoverable reserves of $\sim 600 \mathrm{MMbbl}$ oil and $1400 \mathrm{MMBOE}$ gas (Energy and Building Trends, 2017). This basin is located both onshore and offshore along the west coast of New Zealand's North Island with an area of approximately $330,000 \mathrm{~km}^{2}$ (Fig. 5.1), and comprises up to $10 \mathrm{~km}$ of mid-Cretaceous to recent sedimentary rocks (e.g., King and Thrasher, 1996). The main petroleum source rocks are late Cretaceous-Eocene in age and were deposited in broad, extensive proximal shelf and coastal plain environments. These source rocks were buried in the Neogene to depths where significant oil and gas generation occurred (King and Thrasher, 1996; New Zealand Petroleum and Minerals, 2014). Although commercial petroleum accumulations have been encountered at every stratigraphic level from the Paleocene to Pliocene, trapping mechanisms (structural, stratigraphic or diagenetic) are not well understood (New Zealand Petroleum and Minerals, 2014). Most of the petroleum accumulations are trapped in fault-related anticlines that formed in the last 30 to $40 \mathrm{Myr}$ following compression 
associated with subduction of the Pacific Plate to the east of New Zealand (King and Thrasher, 1996; Stagpoole and Nicol, 2008; Reilly et al., 2015; 2016).

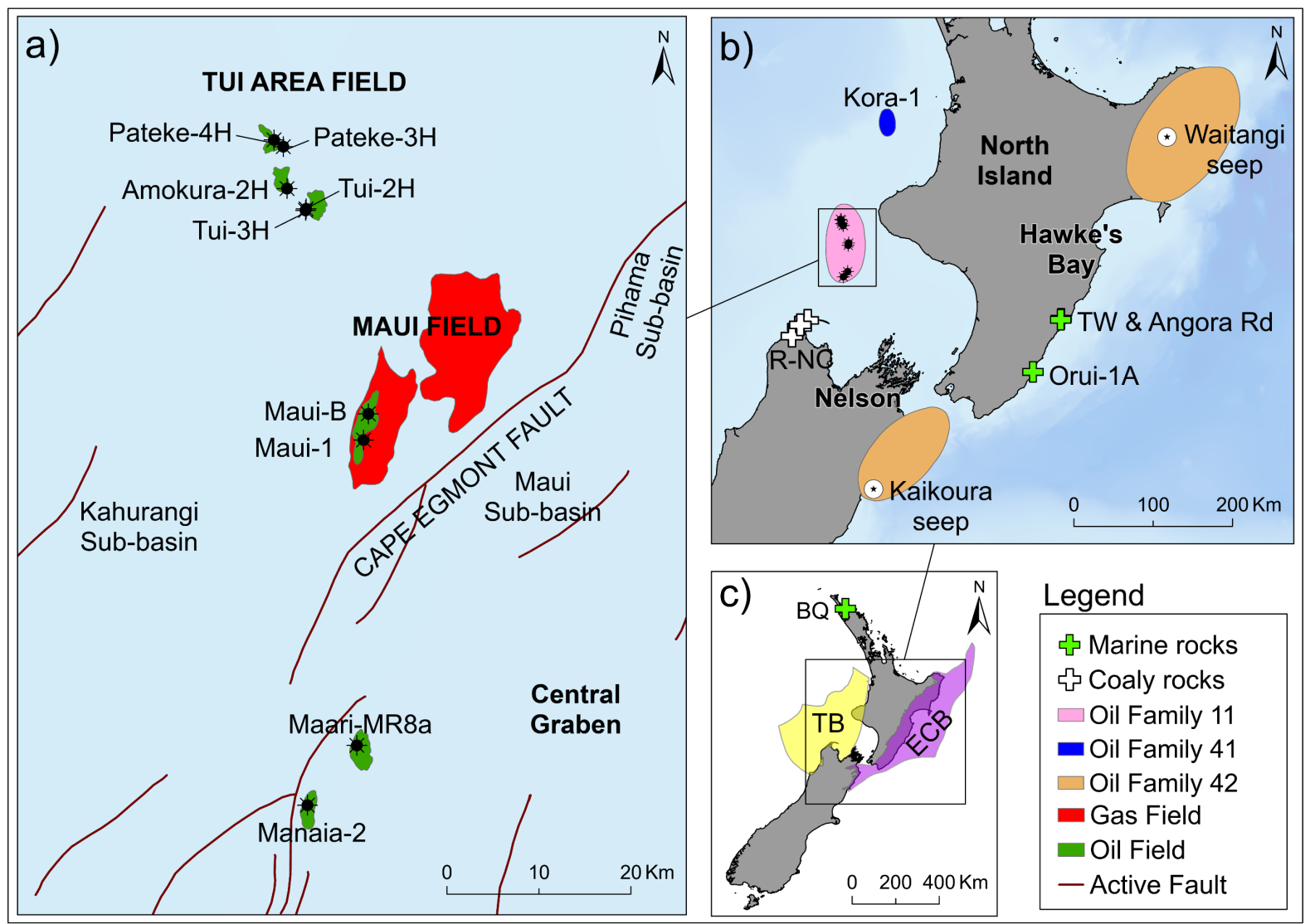

Fig. 5.1. Map of the study area showing locations of the studied oils in the Taranaki and East Coast basins. Also shown are oil and gas fields and major faults bounding late Cretaceous grabens where deposition of the Rakopi and North Cape formations likely occurred (New Zealand Petroleum and Minerals, 2014; Kroeger et al., 2016). TB = Taranaki Basin; ECB = East Coast Basin; R-NC = Rakopi and North Cape formations; TW = Taylor White; $\mathrm{BQ}=$ Blacks Quarry. The white and green crosses indicate locations of samples of the inferred source rocks that have been analysed in Chapter 3 and 4.

There are currently 16 producing oil fields in the Taranaki Basin, both offshore and onshore (King and Thrasher, 1996; New Zealand Petroleum and Minerals, 2014). The Family 11 oils studied here are from the Maui, Tui and Maari-Manaia offshore oil fields (Fig. 5.1a) and are inferred to have been sourced from late Cretaceous coaly rocks of the Rakopi and North Cape formations (Killops et al., 1994; Funnell et al., 2004; Sykes et al., 2012). Regional geological models and seismic facies analyses indicate that these formations are present in the depocenters of the Maui and Pihama sub-basins, which are the potential source kitchens for the Maui, Maari, Manaia and Tui oil fields (Funnell et al., 2004; Kroeger et al., 2016). Further, maturity modelling indicates that the Rakopi and North Cape formations have been generating and 
expelling significant quantities of hydrocarbons from the Late Miocene (20 Ma) to present (Killops et al., 1994; Funnell et al., 2004; Harrison et al., 2013; Kroeger et al., 2016; Pierpont et al., 2017). These hydrocarbons have been discovered in Paleocene-Miocene, stacked terrestrial and marine sandstone reservoirs that are normally referred to as F, D, C and B sands (King and Thrasher, 1996; Funnell et al., 2004). The Paleocene F sands (Farewell Formation) and early Eocene D sands (Kaimiro Formation) were deposited in near-shore, paralic and fluvio-estuarine environments. The middle-late Eocene $\mathrm{C}$ sands (Maui and Mangahewa formations) consist of coastal plain, shoreface, tidal channel and inner shelf sediments, whereas the middle Miocene B sands (Moki Formation) comprise basal floor, slope turbidite, and channel sandstones (King and Thrasher, 1996; Funnell et al., 2004). The Family 11 oils analysed in this study are from the F, D and B sands.

In the northern part of the Taranaki Basin, sub-commercial oils have been discovered in the Kora-1 well that was drilled to target a Miocene aged submarine volcano (King and Thrasher, 1996; Brett, 2005). These oils are genetically grouped under oil Family 41 and are inferred to have been sourced from the late Paleocene Waipawa Formation whose organic matter comprises both marine algae and terrestrial land plants (Reed, 1992; Killops et al., 1994; Clayton, 2011; Sykes et al., 2012; Naeher et al., 2019). The Waipawa Formation is mainly gascondensate-prone because of high contributions of terrestrial organic matter but is inferred to have sourced the Kora oils and a number of oil seeps in the East Coast Basin (Fig. 5.1; Rogers et al., 1999; Naeher et al., 2019). The main reservoirs at the Kora field are the Miocene volcanic rocks and Eocene Tangaroa sandstones, which represent a deep-water submarine fan deposit (Killops et al., 1994; Brett, 2005; Sykes et al., 2012).

\subsubsection{East Coast Basin petroleum system}

The East Coast Basin is located both onshore and offshore on the eastern margin of New Zealand's North Island with an area of about $120,000 \mathrm{~km}^{2}$ (Fig. 5.1a). The basin has numerous onshore hydrocarbon seeps indicating presence of an active petroleum system (Magoon and Dow, 1994; Field et al., 1997). Late Cretaceous-Paleocene sediments of the Whangai and Waipawa formations have been identified as the most promising petroleum source rocks in this basin, but commercial accumulations of oil and gas have not been discovered to date (Field et al., 1997; Hollis and Manzano-Kareah, 2005). The Waipawa Formation is up to $80 \mathrm{~m}$ thick in the basin and has relatively high total organic carbon (TOC; mean of $3.6 \mathrm{wt} \%$ ) and moderate 
hydrogen index values (HI; 22-550 mg HC/g TOC [mean of 245]), indicating a high potential to generate mainly gas or condensate as it lacks oil-prone organofacies (Hollis and ManzanoKareah, 2005; Sykes et al., 2012; Naeher et al., 2019). The formation is typically thermally immature to marginally mature at several locations throughout the basin (Moore, 1989; Field et al., 1997; Hollis and Manzano-Kareah, 2005). The Whangai Formation, on the other hand, is up to $1500 \mathrm{~m}$ thick and comprises four members (Upper Calcareous, Porangahau, Te Uri and Rakauroa) with distinct differences in source rock potential (Hollis and Manzano-Kareah, 2005). The Porangahau and Te Uri members have low oil and gas potential, with average total organic carbon (TOC) and hydrogen index (HI) of $\leq 0.4 \mathrm{wt} \%$ and $\leq 120 \mathrm{mg} \mathrm{HC} / \mathrm{g}$ TOC, respectively. The Rakauroa Member has a higher potential with mean TOC of $0.69 \%$ and mean HI of $164 \mathrm{mg} \mathrm{HC/g}$ TOC. The Upper Calcareous Member exhibits the highest source rock potential, with average TOC and $\mathrm{HI}$ values of $0.56 \%$ and $195 \mathrm{mg} \mathrm{HC} / \mathrm{g}$ TOC, respectively (Hollis and Manzano-Kareah, 2005). The two seep oils studied here (Waitangi and Kaikoura) are genetically grouped under oil family 42, in which the source organofacies exhibit characteristics similar to those of the Whangai Formation (Rogers et al., 1999; Sykes et al., 2012). However, the generally low oil generation potential of this formation does not preclude the possibility that these oils were sourced from a separate Cretaceous source rock with organofacies characteristics similar to those of the Whangai Formation (commonly referred to as 'Whangai-type'; Hollis and Manzano-Kareah, 2005; Sykes et al., 2012; Burton et al., 2019).

\subsection{Methodology}

\subsubsection{Samples}

Thirteen oil samples that are representative of the coaly-sourced Family 11 oils and marinesourced Family 41 and 42 oils were selected from a collection of oils held at GNS Science, Lower Hutt (Table 5.1). Nine samples represent Family 11 oils from the Maui, Tui, Maari and Manaia fields, which broadly represent a $\sim 75 \mathrm{~km}$ sample site across the offshore Taranaki Basin (Fig. 5.1a). The wax contents of these oils range from low $(<19 \%)$ to very high $(>50 \%)$, based on relative abundance of total $n$-alkanes (Manzano-Kareah and Sykes, 2008). These oils show no or slight degree of biodegradation $(\leq 1)$ based on the biodegradation scale of Peters et al. (2005), whereas moderate to high contributions of $\mathrm{C}_{6}$ to $\mathrm{C}_{8}$ branched and cyclic hydrocarbons relative to aromatic hydrocarbons (0.6-1.0) indicates signs of water washing (Sykes and Zink, 2008; Sykes et al., 2012). Two oil samples originate from two different 
reservoirs in the Kora-1 well (Fig. 5.1b). The Kora-1(A) oil is from the Eocene Tangaroa sandstone reservoir whereas the Kora-1(B) oil is from the Miocene volcanic reservoir. These oils are non-waxy $(<19 \%$ total $n$-alkanes) and show a slight level of biodegradation ( $\leq 1$; Peters et al., 2005; Sykes et al., 2012). Additional samples of the Kora-1(A) and Kora-1(B) oils were taken using aliquots from different bottles held at the New Zealand National Core Store in Featherston (Table 5.1). Two oil seep samples originate from the northern (Waitangi) and southern (Kaikoura) parts of the East Coast Basin (Fig. 5.1b). The Waitangi oil has a medium wax content (29-40\%) with slight to moderate levels of biodegradation (biomarker scale of 12; Peters et al. 2005), whereas the Kaikoura oil is heavily biodegraded (biomarker scale of 3+) and contains no wax (Rogers et al., 1999; Peters et al., 2005; Sykes et al., 2012).

Re and Os concentrations and isotopic compositions of samples representative of potential source rocks that generated the oils studied here (Rakopi, North Cape, Whangai and Waipawa formations) are provided in Chapters 3 and 4 (Tables 3.2 and 4.1). The samples from Rakopi and North Cape formations comprise fluvio-deltaic coals and coaly mudstones that were collected at outcrop locations in Collingwood district, northwest Nelson (Fig. 5.1b). The marine mudstones of the Waipawa and Whangai formations were obtained from a drill core (Orui-1a) in coastal Wairarapa and outcrop locations (Taylor White section, Angora Road and Blacks Quarry) in the southern Hawke's Bay and Northland regions of New Zealand's North Island (Fig. 5.1b).

\subsubsection{Asphaltene precipitation}

The asphaltene fraction contains the majority (up to 98\%) of Re and Os in crude oil, and in most cases possesses Re-Os isotopic compositions that are representative of the whole oil (Selby et al., 2007a; Georgiev et al., 2016; DiMarzio et al., 2018; J. Liu et al., 2019). Separation of this fraction, therefore, pre-concentrates the elements and allows for more precise Re and Os analyses to be undertaken. This is especially important for the New Zealand coaly-sourced oils, which typically have very low asphaltene contents $(<3 \%)$.

Asphaltenes were precipitated from the bulk oil samples using methods reported in Speight (2004) and Selby et al. (2007a). Stock oils were thoroughly mixed to remove heterogeneities due to storage density segregation. Many of the coal-sourced crude oils from New Zealand are solid at room temperature due to their medium to high wax content (29- $\geq 50 \%)$ and abundant 
terrestrial organic matter (Sykes et al., 2012) Therefore, the first step in the separation process involved heating the solid stock oils to about $50^{\circ} \mathrm{C}$ to obtain a viscous liquid that was thoroughly mixed prior to sub-sampling. The subsamples $(\sim 1 \mathrm{~g})$ were placed in pre-heated glass vials where an excess of $n$-heptane $(40 \mathrm{ml})$ was added and thoroughly mixed before continuous, gentle agitation overnight (at least $12 \mathrm{~h}$ ) at room temperature using an orbital shaker. The contents of the vials were then centrifuged at $3500 \mathrm{rpm}$ for 10-15 minutes to allow complete separation of precipitated asphaltene and soluble maltene fractions. Maltene fractions were decanted to waste while the remaining residues (asphaltene fractions) were transferred into pre-weighed glass vials and dried overnight on a hot plate at $60{ }^{\circ} \mathrm{C}$. Depending on asphaltene content, the above steps were repeated 2 to 30 times per sample to obtain at least $\sim 250 \mathrm{mg}$ of asphaltene required for Re-Os analysis.

\subsubsection{Re-Os analysis}

Rhenium and Os analysis of the asphaltene fractions was undertaken at Durham University's laboratory for Source Rock and Sulfide Geochronology and Geochemistry following previously published protocols (e.g., Selby et al., 2007a; J. Liu et al., 2019). In brief, asphaltene fractions (150-200 mg) were transferred into Carius tubes using small amounts of chloroform $\left(\mathrm{CHCl}_{3} ; \leq 1 \mathrm{ml}\right)$ and dried on a hot plate at $60{ }^{\circ} \mathrm{C}$ overnight to evaporate the $\mathrm{CHCl}_{3}$. After complete solvent removal, a known amount of a mixed ${ }^{190} \mathrm{Os}+{ }^{185} \mathrm{Re}$ tracer solution and inverse aqua-regia digestion mixture $\left(3 \mathrm{ml} 12 \mathrm{~N} \mathrm{HCl}+6 \mathrm{ml} 15.5 \mathrm{~N} \mathrm{HNO}_{3}\right)$ were sequentially added, the tube sealed and then placed in an oven at $220^{\circ} \mathrm{C}$ for $24 \mathrm{~h}$. The Os fraction was extracted and purified from the inverse aqua-regia solution using solvent extraction $\left(\mathrm{CHCl}_{3}\right.$ and back extraction in $\mathrm{HBr}$ ) and micro-distillation methods, respectively, with the Re fraction purified from the Os-extract inverse aqua-regia solution using $\mathrm{NaOH}$-acetone solvent extraction and anion exchange chromatography $\left(\mathrm{HCl}-\mathrm{HNO}_{3}\right)$. The obtained Re and Os fractions were then loaded onto nickel and platinum wire filaments, respectively, and their isotopic compositions measured on the Thermo Scientific TRITON negative thermal ionisation mass spectrometer (N-TIMS; Creaser et al., 1991) housed at the Arthur Holmes Laboratory, Durham University. The total procedural blanks in the course of this study were $2.41 \pm 0.07 \mathrm{pg}$ Re and $0.12 \pm 0.05$ pg Os, with an average ${ }^{187} \mathrm{Os} /{ }^{188} \mathrm{Os}$ of $0.26 \pm 0.01(2 \mathrm{SD}, \mathrm{n}=4)$. The average ${ }^{185} \mathrm{Re} /{ }^{187} \mathrm{Re}$ value for the Re standard solution (125 pg aliquot) was $0.5989 \pm 0.0016$ ( $2 \mathrm{SD}, \mathrm{n}=87$ ), which is in excellent agreement with previously published data (e.g., Cumming et al., 2014; J. Liu et al., 
2019). The average ${ }^{187} \mathrm{Os} /{ }^{188}$ Os value for the in-house Durham Romil Osmium Standard (DROsS -50 pg aliquot) was $0.16084 \pm 0.00043(2 \mathrm{SD}, \mathrm{n}=8)$, consistent with previously published data from other laboratories $(0.16078 \pm 0.00024$, Liu and Pearson, 2014; $0.16091 \pm 0.00015$, van Acken et al., 2019). The uncertainties reported here include those from asphaltene weighing, blank elemental and isotopic compositions, spike calibration, and the precision of the repeated standard measurements.

\subsection{Results}

Twelve of the thirteen oil samples analysed in this study have low asphaltene contents, ranging between 0.9 to $2.4 \%$. The asphaltene content is notably higher $(15.5 \%)$ in the Kaikoura Seep oil. For all samples, there is no direct relationship between the asphaltene content and Re and Os concentrations in the asphaltene, which range from 0.18 to $5.90 \mathrm{ppb}$ Re, 3.4 to $400.2 \mathrm{ppt}$ Os and 1.3 to $149.9 \mathrm{ppt}{ }^{192} \mathrm{Os}$ (a measure of Os concentration unaffected by radiogenic ingrowth of ${ }^{187} \mathrm{Os}$; Fig. 5.2a; Table 5.1). Rhenium and ${ }^{192}$ Os concentrations in the coaly-sourced Family 11 oils range from 0.18 to $0.45 \mathrm{ppb}$ and 1.3 to $12.7 \mathrm{ppt}$, respectively, mirroring the low concentrations of these elements in their potential Rakopi and North Cape source rocks $(0.19$ $0.37 \mathrm{ppb}$ Re and 5.3-9.6 ppt ${ }^{192} \mathrm{Os}$; Fig. 5.2b). The low Re and Os concentrations in these samples coupled with limited asphaltene contents made precise measurements difficult, with blank corrections ranging from 3.8 to $10.5 \%$ for Re and 1.2 to $21.1 \%$ for Os. In contrast, the asphaltene fractions for the marine oils (Families 41 and 42) exhibit Re (0.17-5.90 ppb) and ${ }^{192}$ Os (5.3-149.9 ppt) concentrations that range to much higher values (Fig. 5.2a). The aliquots of the Kora-1 oils from different bottles show variations in Re and Os concentrations, with the oils from the bottles at GNS Science having lower Re and Os content than those from the New Zealand National Core Store (Fig. 5.2a; Table 5.1). The ${ }^{187} \mathrm{Os} /{ }^{188}$ Os for these replicate analyses are, however, reproducible within uncertainty (Table 5.1). The ${ }^{187} \mathrm{Re} /{ }^{188}$ Os values for the Kora(B) replicates are also reproducible within uncertainty, but those of the Kora-1(A) oil show a larger variation (Table 5.1). 


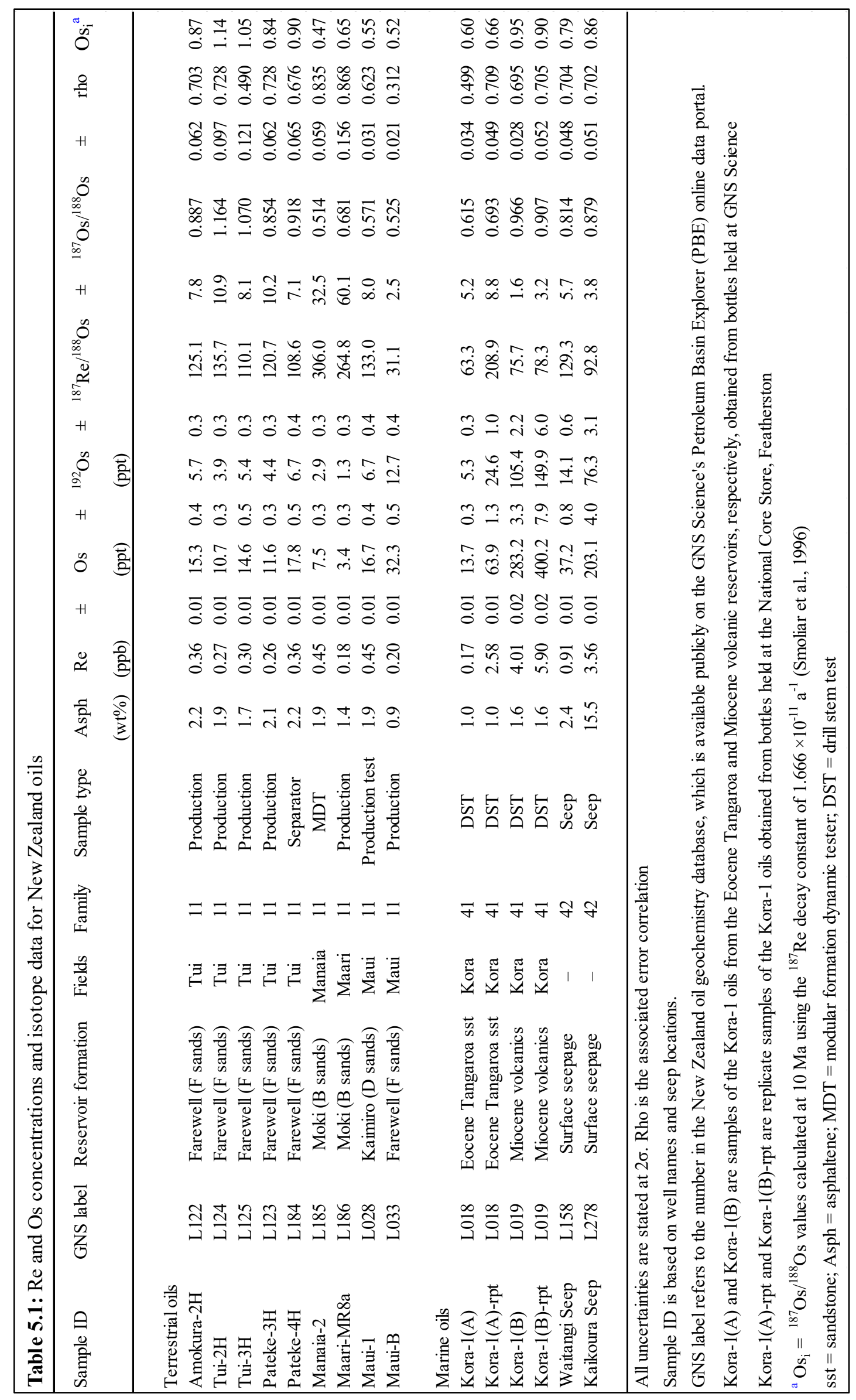




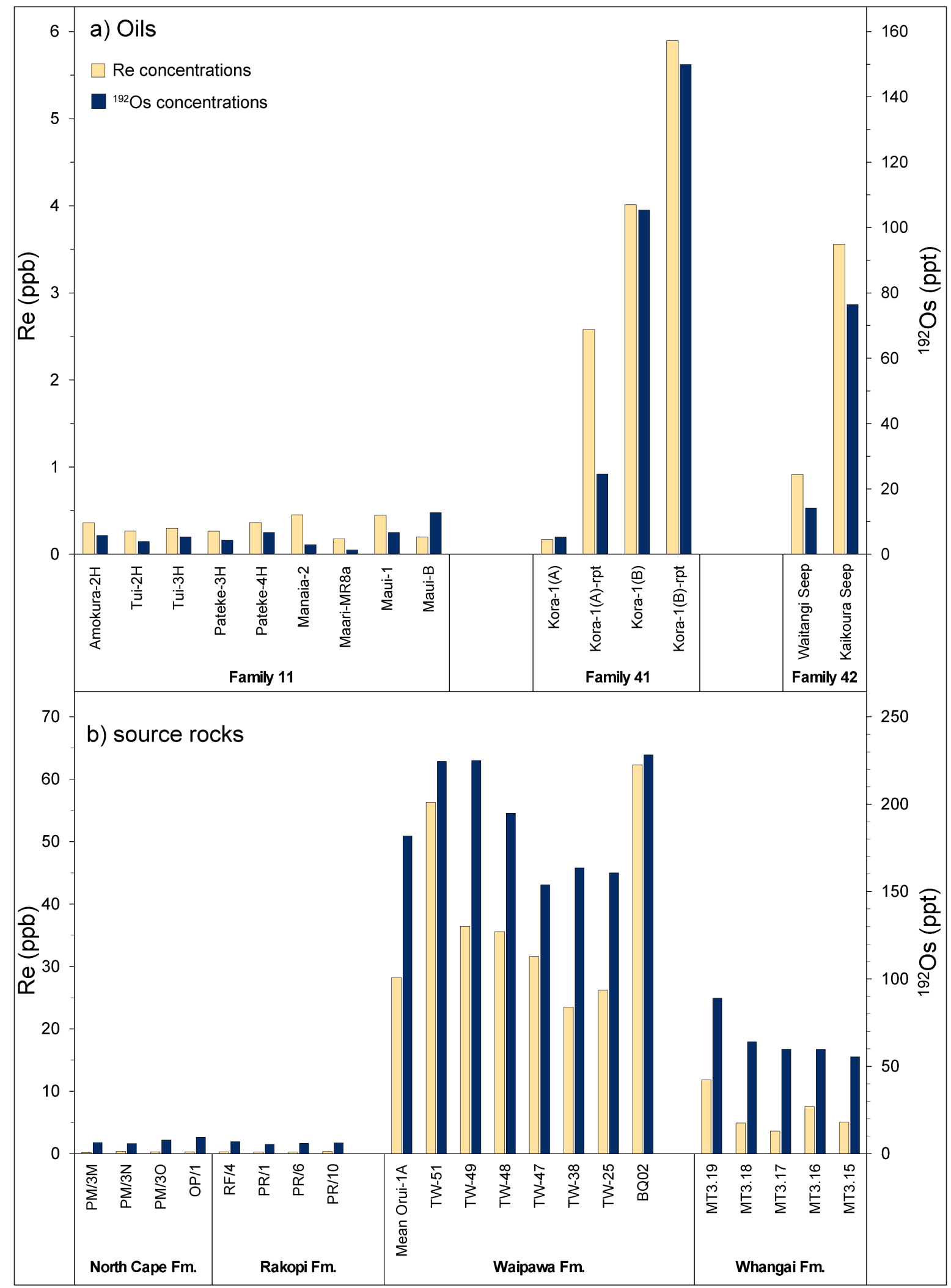

Fig. 5.2. Re and Os concentrations in a) the asphaltene fractions of Families 11,41 and 42 oils and b) their potential source rocks from the Taranaki and East Coast basins. The concentrations of Re and Os in the oils appear to reflect those of the source units. Note the difference in scale between the two plots. The legend in Figure 5.2a is used for both plots. 
The asphaltene fractions of Family 11 oils have ${ }^{187} \mathrm{Re} /{ }^{188}$ Os ratios ranging from 31.1 to 306.0 and ${ }^{187} \mathrm{Os} /{ }^{188} \mathrm{Os}$ ratios ranging from 0.51 to 1.16 . These ratios show no recognisable linear trend on an isochron diagram (Fig. 5.3). Within this oil family, the Tui area oils exhibit higher (i.e., more radiogenic) ${ }^{187} \mathrm{Os} /{ }^{188} \mathrm{Os}$ ratios $(0.85-1.16)$ and a smaller range in ${ }^{187} \mathrm{Re} /{ }^{188} \mathrm{Os}$ ratios (108.6-135.7) compared to those from the Maui and Manaia-Maari fields (0.51-0.68 and 31.1306.0, respectively; Fig. 5.3). The ${ }^{187} \mathrm{Re} /{ }^{188} \mathrm{Os}$ and ${ }^{187} \mathrm{Os} /{ }^{188} \mathrm{Os}$ values for the asphaltene fractions of the marine oils from Families 41 and 42 oils range from 63.3 to 208.9 and 0.62 to 0.97 , respectively.

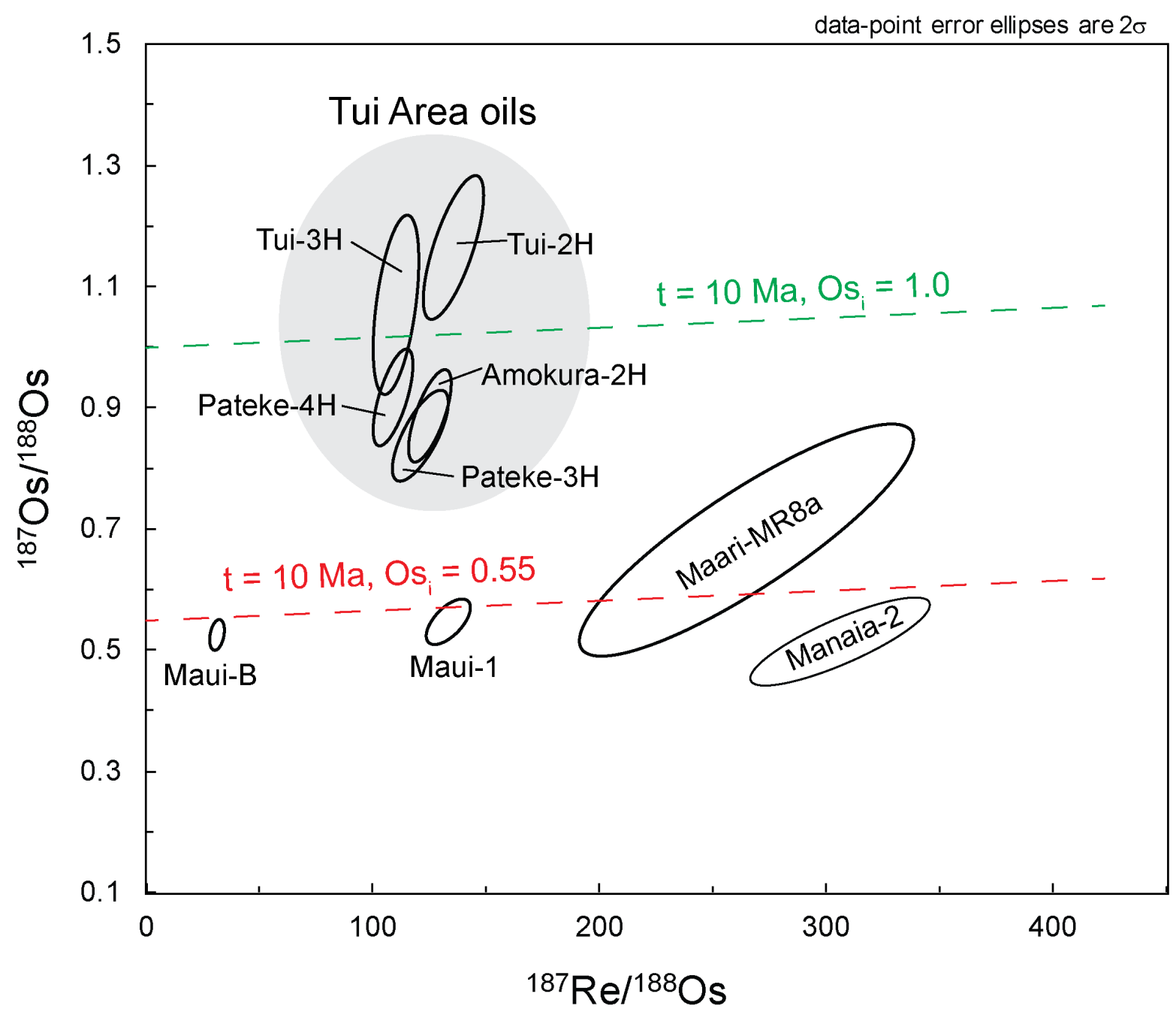

Fig. 5.3. ${ }^{187} \mathrm{Re} /{ }^{188} \mathrm{Os}$ versus ${ }^{187} \mathrm{Os} /{ }^{188} \mathrm{Os}$ for the asphaltene fractions of Family 11 oils. The Tui area oils cluster together with relatively higher ${ }^{187} \mathrm{Os} /{ }^{188} \mathrm{Os}$ ratios and a small range in ${ }^{187} \mathrm{Re} /{ }^{188} \mathrm{Os}$ ratios. Reference lines with slopes equivalent to the approximate generation age of the oil samples (10 Ma) and $\mathrm{Os}_{\mathrm{i}}$ values of 0.55 and 1.0 are shown by the red and green dashed lines, respectively. 


\subsection{Discussion}

\subsubsection{Re and Os systematics of coaly-sourced Family 11 oils}

The Family 11 oils were analysed to evaluate if Re-Os geochronology could be applied to oils generated from terrestrial coals, and if their initial Os isotope compositions can be traced back to their source rocks. The ${ }^{187} \mathrm{Re} /{ }^{188} \mathrm{Os}$ and ${ }^{187} \mathrm{Os} /{ }^{188} \mathrm{Os}$ ratios in these samples do not show any linear relationship on an isochron diagram (Fig. 5.3), indicating a more complex relationship. Three criteria must be met before reliable Re-Os isochron dates can be produced: 1) the Re-Os isotopic systematics must remain in a closed system, 2) the Osi must be similar, and 3) there must be a sufficient spread in ${ }^{187} \mathrm{Re} /{ }^{188}$ Os ratios to define an isochron (Cohen et al., 1999).

Experimental studies have demonstrated that $\mathrm{Re}$ and Os can readily transfer from aqueous solutions to oils, raising the possibility that mixing of oil with formation waters in petroleum reservoirs may disturb Re-Os systematics of the oils (Mahdaoui et al., 2015; Hurtig et al., 2019). If this process occurs in natural systems, low Re- and Os-bearing oils such as those of Family 11 are likely to be adversely affected because water-oil ratios of less than 100 (based on estimated concentration levels of $4 \mathrm{ppt} \mathrm{Re}$ and $0.07 \mathrm{ppt}$ Os in groundwaters) would be required to completely overprint their original Os isotopic signature (e.g., Colodner et al., 1993; Paul et al., 2010; Mahdaoui et al., 2015). Such water-oil ratios are very likely in petroleum systems because reservoir rocks are often fully saturated with formation waters prior to migration of oil (Magoon and Dow, 1994; Levorsen, 2001). However, recent field studies have suggested that the behaviour of Re and Os in natural oil-water systems may differ from observations in laboratory experiments (Liu et al., 2018; Hnatyshin et al., 2019). Samples of deep (118-3310 m), natural groundwaters from major aquifers of the Williston Basin in the Western Canada Sedimentary Basin show evidence that isotopic equilibrium between oil and water has not been reached (Hnatyshin et al., 2019). Similarly, crude oils from the Duvernay petroleum system in the Western Canada Sedimentary Basin, with asphaltene, Re and Os contents comparable to those of Family 11 oils (10 out of 14 samples have $<6 \%$ asphaltene, $<1$ ppb Re and $<8$ ppt ${ }^{192} \mathrm{Os}$ ) were shown to have been largely unaffected by interaction with reservoir waters (Liu et al., 2018). The ${ }^{187} \mathrm{Os} /{ }^{188} \mathrm{Os}$ in these oils are much lower than estimated ${ }^{187} \mathrm{Os} /{ }^{188} \mathrm{Os}$ values for formation fluids in the basin, and thus, likely reflect those of potential source rock at the time of generation (Liu et al., 2018). A kinetic barrier that inhibits Re transfer at the water-oil interface has also been identified in aqueous solutions with low $\mathrm{Re}$ 
concentrations $(<2.2 \mathrm{ppb})$, such as is the case for most reservoir waters (Hurtig et al., 2019). Below this concentration, Re transfer between water and oil is not experimentally observed. The existence of a similar kinetic barrier for Os transfer was not observed within the experimental conditions of Hurtig et al. (2019). However, it is unlikely that the Os systematics of the Family 11 oils were severely impacted by interaction with reservoir waters because oils produced from the same reservoir interval exhibit different ${ }^{187} \mathrm{Os} /{ }^{188} \mathrm{Os}$ (e.g., Tui area oils vs Maui-B [F sands]; Table 5.1), and oils produced from different reservoirs show comparable Os isotope compositions (Maui-B [F sands] vs Maui-1 [D sands] vs Manaia-2 [B sands]; Table 5.1). Therefore, despite the low concentrations, it appears that the Re-Os systematics of Family 11 oils likely reflect those inherited from their source rocks.

If the Re-Os systematics of Family 11 oils have remained closed, then the scatter on the isochron diagram could be attributed to heterogeneous Osi. Two factors may have caused variation in Osi of Family 11 oils: 1) the generally heterogeneous nature of the Rakopi and North Cape coaly sediments that potentially sourced these oils and/or 2) a complex and lengthy oil generation history. The Rakopi and North Cape formations are vertically extensive (up to $3000 \mathrm{~m}$ thick) with varying lithofacies associations (Killops et al., 1994; Sykes et al., 2004; Browne et al., 2008; Higgs et al., 2010). Given that at least $1000 \mathrm{~m}$ of a source rock unit can lie within the oil window at any given time (Killops et al., 1994), it is possible that these formations have generated oils with varying Osi values. The Taranaki Basin also records a complex basin evolution history from the break-up of Gondwana in the Cretaceous to convergence of plate boundaries in the Neogene (King and Thrasher, 1996; Kroeger et al., 2016). This had a significant impact on thermal maturation of source rock kerogen and generation of petroleum in the basin (Kroeger et al., 2016; Reilly et al., 2016). Maturity modelling of two major regional kitchens that likely sourced the Family 11 oils (Maui and Pihama sub-basins) predicts that expulsion of oil from the coaly source rocks started in the Miocene (20 Ma) and continues until the present day, with significant expulsion happening in the last 10 Ma (Funnell et al., 2001; 2004; Reilly et al., 2016; Pierpont et al., 2017). Such a lengthy oil generation history would be expected to produce variations in Osi values (Lillis and Selby, 2013; Cumming et al., 2014), and thus, may explain the observed scatter in the oils' ${ }^{187} \mathrm{Re} /{ }^{188}$ Os and ${ }^{187} \mathrm{Os} /{ }^{188}$ Os ratios (Fig. 5.3). 
The Family 11 oil samples were selected to represent crude oils from different locations of the Taranaki Basin to maximise the likelihood of obtaining a sufficient spread in ${ }^{187} \mathrm{Re} /{ }^{188} \mathrm{Os}$ ratios to generate an isochron (Fig. 5.1a; Selby and Creaser, 2005a). The ${ }^{187} \mathrm{Re} /{ }^{188}$ Os values for the asphaltene fractions of these oils, however, vary by only 275 units (Table 5.1), which is low when compared to most oils successfully dated using the Re-Os geochronometer (up to 1500 units; e.g., Selby et al., 2005a; Selby and Creaser, 2005a; Finlay et al., 2011; Lillis and Selby, 2013; Cumming et al., 2014; Liu et al., 2018). Asphaltenes derived from South Australian asphaltites have produced reliable Re-Os oil isochrons with a much lower spread in ${ }^{187} \mathrm{Re} /{ }^{188} \mathrm{Os}$ ratios ( 147 units) than the Family 11 oils (Corrick et al., 2019; Scarlett et al., 2019). However, these asphaltites were generated in the mid-Cretaceous (104 $\pm 12 \mathrm{Ma}$; Scarlett et al., 2019) and would have, thus, radiogenically evolved to better define an isochron compared to Family 11 oils that are still being generated to date. Therefore, the lack of an isochron fit in the Family 11 oils appears to have resulted from a combination of large variations in Osi values, relatively small variations in ${ }^{187} \mathrm{Re} /{ }^{188} \mathrm{Os}$ values, and young and protracted generation ages.

\subsubsection{Do the Os isotopes of Family 11 oils reflect those of potential source rocks?}

Upon thermal maturation of kerogen, the ${ }^{187} \mathrm{Os} /{ }^{188} \mathrm{Os}$ of the source rock is transferred to the generated oil (Selby and Creaser, 2005a; Selby et al., 2007a; Finlay et al., 2011; Rooney et al., 2012; Cumming et al., 2014; Liu et al., 2018). The Osi values of the oils should, therefore, reflect the ${ }^{187} \mathrm{Os} /{ }^{188} \mathrm{Os}$ of the source rock at the time of oil generation $(\mathrm{Osg})$, which would form the basis for Os isotope fingerprinting of oils to their source rock. Given that oil generation has occurred over a long period of time (20 Ma - present) in the Taranaki basins (Funnell et al., 2001; 2004; Reilly et al., 2016; Pierpont et al., 2017), 10 Ma is considered in this study as the average oil generation date for the purpose of calculating the Osi and Osg values from the present-day Re-Os data. The use of an average oil generation date does not affect the outcome of Os isotope fingerprinting because Osi and Osg values generally covary or show little variations with changing oil generation dates, especially given the ${ }^{187} \mathrm{Re} /{ }^{188} \mathrm{Os}$ values exhibited by both the oils and source rocks are low ( $\leq 306$ for the samples studied here; Cumming et al., 2014).

Overall, the Osi values for Family 11 oils range from 0.47 to 1.14 , with a mean value of 0.78 (Fig. 5.4a). These values overlap well with Osg values $(0.38-1.26, \overline{\mathrm{x}}=0.81)$ for the late Cretaceous Rakopi and North Cape formations, which are considered potential source rocks 
for the oils (Sykes et al., 2012). Previous oil-oil and oil-source correlations of the Family 11 oils have mainly relied on variations in the degree of marine influence, anoxicity and relative abundance of higher plant biomarkers (terpanes; Killops et al., 1994; 1995; Sykes et al., 2012). While abundant diterpanes have been attributed to the dominance of gymnosperm plants during the late Cretaceous, higher contributions of triterpanes indicate the increasing dominance of angiosperms during the Eocene (Killops et al., 1995). Diterpanes are consistently high in Family 11 oils compared to other coaly-sourced oils in the Taranaki Basin, indicating derivation from source rocks that are late Cretaceous in age (Killops et al., 1994; Sykes et al., 2012). Therefore, the similarity of the Osi for Family 11 oils and the Osg of the late Cretaceous Rakopi and North Cape formations is consistent with the previous oil-source correlations that suggest these units to be the source of the oils (Killops et al., 1994; Sykes and Zink, 2008; Sykes et al., 2012).

Within the Family 11 oils, the Tui area oils exhibit notably higher (i.e., more radiogenic) Osi values (0.84-1.14) than oils from the Maui, Manaia and Maari fields with moderately radiogenic Osi values $(0.47-0.65)$. This is consistent with previous suggestions that the Tui area oils are compositionally different from other Family 11 oils, to the extent that they are clearly sourced from a different kitchen (Sykes and Zink, 2008; Sykes et al., 2012). The distinctive nature of the Tui area oils stems from their moderately high level of marine influence (based on tricyclic terpane, C27 sterane and drimane biomarkers), heavy carbon isotope profiles for $n$-alkanes $\left(\mathrm{C}_{12}-\mathrm{C}_{29}\right)$ and lower aromatic hydrocarbon-derived thermal maturities (Manzano-Kareah and Sykes, 2008; Sykes and Zink, 2008; Sykes et al., 2012). Despite the evidence of marine influence, none of the Tui oils shows elevated Re and Os contents comparable to those seen in the marine Kora-1 oils (Fig. 5.2a), which is consistent with a lack of enrichment of these elements in marine-influenced intervals of their potential source rocks (Chapter 4). The high Osi values (0.84-1.14) for the Tui area oils correlate more to Osg values of the Rakopi Formation (0.92-1.38; $\mathrm{n}=4$; Fig. 5.4a), suggesting likely derivation from this unit. In contrast, the Maui, Manaia and Maari oils (Osi values $=0.47-0.65$ ) seem to correlate more with the North Cape Formation which has Osg values ranging from 0.38 to $0.52(n=4)$, but with possible contributions from the Rakopi Formation (Fig. 5.4a). Except for the Maui oils, these results are in general agreement with previous findings that the Tui oils are likely sourced from lower intervals of the Rakopi Formation, whereas the Maari-Manaia oils are likely sourced either from the upper Rakopi Formation or the North Cape Formation 
(Sykes and Zink, 2008). This interpretation was based on biomarker results which show that the Tui area oils contain relatively higher gymnosperm- and lower angiosperm-plant contributions compared to oils in the Maari-Manaia fields (Sykes and Zink, 2008).

a) Terrestrial samples

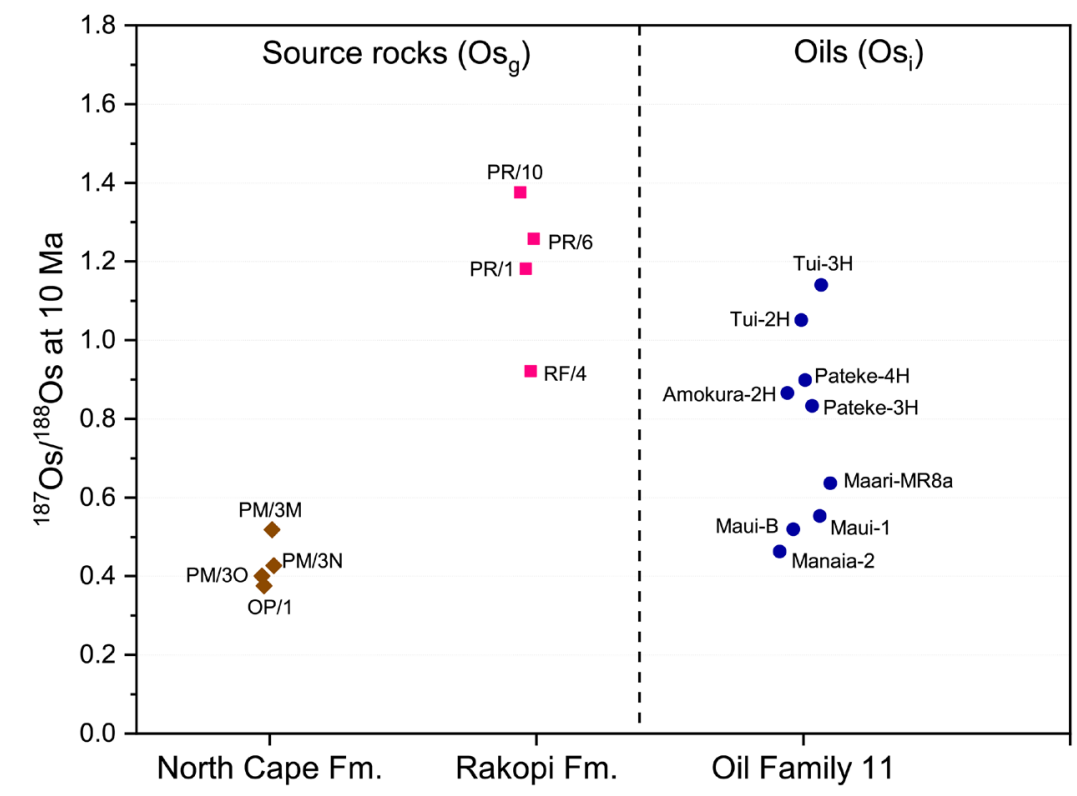

b) Marine samples

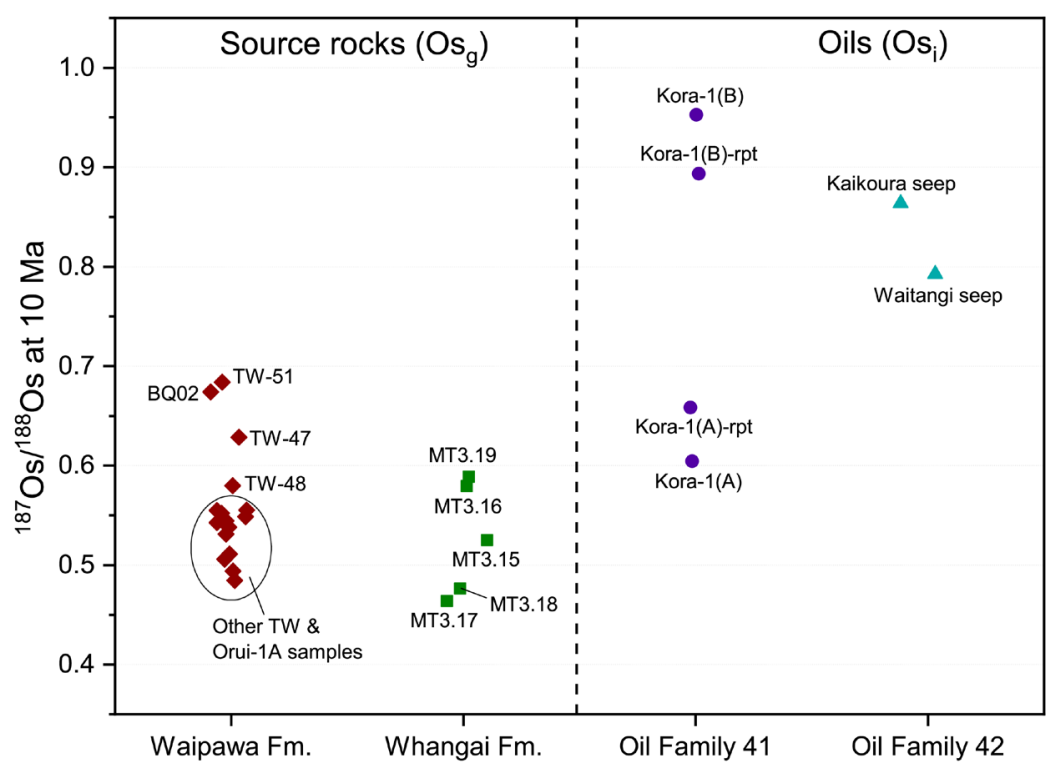

Fig. 5.4. A comparison of ${ }^{187} \mathrm{Os} /{ }^{188} \mathrm{Os}$ at the time of oil generation (estimated to be $\sim 10 \mathrm{Ma}$ ) for a) Family 11 oils and their potential source rocks, the Rakopi and North Cape formations from the Taranaki Basin and b) oil Families 41 and 42 and their potential source rocks, the Whangai and Waipawa formations. Fm. = Formation. 
Although the Os isotopes appear to link Family 11 oils to the late Cretaceous Rakopi and North Cape formations and show a clear distinction between the Tui area oils and the other Family 11 oils, it is important to note that due to stratigraphic variations in Re concentrations and initial ${ }^{187} \mathrm{Os} /{ }^{188} \mathrm{Os}$ of the source units, Osi values in crude oils can sometimes vary significantly even for oils with a single source and simple thermal maturation history. For example, the Osi values for the marine Duvernay oil in the Western Canada Sedimentary Basin vary from 0.55 to 1.06 $(\mathrm{n}=14)$ synonymous with Osg values $(0.46-1.48)$ of its known single source, the Duvernay Formation, which has undergone a simple thermal maturation history during the Laramide orogeny (Liu et al., 2018). The study by Liu et al. (2018) demonstrated the importance of a large number of samples from the presumed source rock formation $(n=33)$ over a large geographical area $\left(45,000 \mathrm{~km}^{2}\right)$ for robust Os isotope fingerprinting, especially where the difference in ${ }^{187} \mathrm{Os} /{ }^{188} \mathrm{Os}$ of potential source units are unlikely to be significant due to, for instance, small differences in depositional ages.

\subsubsection{Re-Os systematics of marine Families 41 and 42 oils}

\subsubsection{Family 41 oils}

The Family 41 oils are inferred to have been sourced from the Waipawa Formation, which is modelled in the Kora field as reaching thermal maturity for oil expulsion from the Miocene (15 Ma) to present day (Brett, 2005; Clayton, 2011; Sykes et al., 2012). An estimated age of $10 \mathrm{Ma}$ was therefore used to calculate Osi and Osg values for the oils and source rock, respectively. The results show that the two Family 41 oils exhibit notably different Osi values; the Kora1(A) oil from the Eocene Tangaroa sandstone reservoir has a moderately radiogenic Osi value (0.63; mean of replicate analyses) that is indistinguishable from the Osg values (0.48-0.68) of the inferred Waipawa Formation source rock (Fig. 5.4b). In contrast, the Osi value (0.92; mean of replicate analyses) for the Kora-1(B) oil from the Miocene volcanic reservoir is more radiogenic, suggesting one of three possible scenarios: 1) the oil was sourced from a different interval of the Waipawa Formation to that which sourced the Kora-1(A) oil, 2) the oil was derived from an entirely different source rock, or 3) the Re-Os systematics of the oil have been disturbed.

It is unlikely that the Kora-1(B) oil was sourced from a different interval of the Waipawa Formation because the formation is thin ( $<30 \mathrm{~m}$ in the vicinity of Kora field) and was deposited over a short period of time ( $\sim 700 \mathrm{Kyr})$, capturing minimal variations in seawater ${ }^{187} \mathrm{Os} /{ }^{188} \mathrm{Os}$ 
(Clayton, 2011; Hollis et al., 2014; Naeher et al., 2019; Chapter 3). Based on Re-Os data from samples that are representative of a greater proportion of the formation's thickness $(\sim 80 \mathrm{~m})$ in the East Coast Basin, these ${ }^{187} \mathrm{Os} /{ }^{188}$ Os are unlikely to have radiogenically evolved to 0.92 by $10 \mathrm{Ma}$ (present-day ${ }^{187} \mathrm{Os} /{ }^{188} \mathrm{Os}=0.53-0.76$; Table 3.2; Hollis et al., 2014; Naeher et al., 2019; Chapter 3). In addition, the small stratigraphic thickness means that the whole formation would most certainly lie within the oil generation window at the same time (Killops et al., 1994).

The suggestion that a different marine unit may have sourced the Kora-1(B) oil is not implausible given that 3D migration modelling of the Kora field indicates that oil charge from the Waipawa Formation to the Miocene volcanic reservoir hosting the oil is highly limited (Clayton, 2011). This is due to 1) the presence of the underlying Tangaroa sandstone reservoir which would trap any vertical migration of oils, and 2) a reliance on at least $2000 \mathrm{~m}$ of vertical migration (Brett, 2005; Clayton, 2011). Potentially there are faults crossing the sandstone reservoir and its top seal (Tikorangi limestone) which would provide conduits for the oil, but these are not continuous and would only provide very local access to the overlying strata (Clayton, 2011). The source kerogen for the Kora-1(B) oil also exhibits relatively lower gymnosperm and higher angiosperm inputs compared to the Kora-1(A), indicating a likely derivation from a source rock that is younger than the Waipawa Formation (Killops et al., 1995; Sykes and Zink, 2012; Sykes et al., 2012). However, such a source rock should exhibit the unique characteristics of the oil, which is: having both marine and terrestrial organic matter, heavy carbon isotope signature and exceptionally high $\mathrm{C}_{30}$ sterane content (Rogers et al., 1999; Hollis and Manzano-Kareah, 2005; Sykes et al., 2012). Apart from the Waipawa Formation, no other single source rock unit with these features has been identified in New Zealand's sedimentary basins (Hollis et al., 2014). Therefore, rather than having a different source, it is more likely that the Re-Os systematics of the Kora-1(B) have been disturbed or represent a mixture of sources where the diagnostic Waipawa Formation signature is still dominant but with contribution from a different source with higher ${ }^{187} \mathrm{Os} /{ }^{188} \mathrm{Os}$.

Interaction with reservoir waters is one of the ways that Re-Os systematics of an oil may be disturbed (Mahdaoui et al., 2015; Hurtig et al., 2019). These groundwaters derive their Re and Os from interactions with sedimentary rocks (Mahdaoui et al., 2015) and would, therefore, exhibit ${ }^{187} \mathrm{Os} /{ }^{188} \mathrm{Os}$ comparable to those of the reservoir. Given that most volcanic rocks have non-radiogenic ${ }^{187} \mathrm{Os} /{ }^{188} \mathrm{Os}$ (generally $<0.3$; e.g., Lassiter and Luhr, 2001; Suzuki and Tatsumi, 
2006), formation waters in the Miocene volcanic reservoir hosting the Kora-1(B) oil should display less radiogenic ${ }^{187} \mathrm{Os} /{ }^{188} \mathrm{Os}$ than in the Eocene sandstone reservoir, assuming an Eocene seawater ${ }^{187} \mathrm{Os} /{ }^{188} \mathrm{Os}$ for the formation waters in the latter $(\sim 0.5$; e.g., Pegram and Turekian, 1999). Consequently, the Kora-1(B) oil should exhibit less radiogenic ${ }^{187} \mathrm{Os} /{ }^{188}$ Os values than the Kora-1(A) oil if its Re-Os systematics have been overprinted by interaction with the formation waters. Instead, the opposite is observed, with the Kora-1(B) oil having more radiogenic Osi value (0.92) than the Kora-1(B) oil (0.63). Therefore, it is unlikely that interaction with formation fluids has significantly disturbed the Re-Os isotope systematics of these oils.

A post-accumulation process that could have more likely altered the Re-Os isotope systematics of the Kora-1(B) oil is thermochemical sulfate reduction (TSR; Lillis and Selby, 2013). TSR is a redox reaction that occurs in reservoirs containing anhydrite $\left(\mathrm{CaSO}_{4}\right)$ or another source of sulfate, whereby petroleum is oxidized to carbon dioxide and sulfate is reduced to hydrogen sulfide (Goldstein and Aizenshtat, 1994; Walters et al., 2015). Sulfate minerals are known to occur in many volcanoes, resulting largely from mixing of sulfur-bearing magmatic ash with groundwater (Hattori, 1996; Zimbelman et al., 2005; Minami et al., 2016). Therefore, it is likely that anhydrite or another form of sulfate is present in the Miocene volcanic reservoir hosting the Kora-1(B) oil. Oils in Bighorn Basin, USA are interpreted to have been affected by TSR based on high triaromatic steroids (TAS) ratios and dominance of dibenzothiophene and several low molecular weight aromatic compounds, specifically toluene, xylene and benzene (Bjorøy et al. (1996). The Kora-1(B) oil has a relatively higher TAS value (0.45) compared to the Kora-1(A) (0.37; Sykes et al., 2012). Although the dibenzothiophene content is higher in the Kora-1(A) oil than in Kora-1(B), the low molecular weight aromatic compounds are more than an order of magnitude higher in the Kora-1(B) than in Kora-1(A) (Sykes et al., 2012), indicating possible alteration by TSR. Oil from the Bighorn Basin that have experienced TSR exhibit much higher Osi values $(1.88 \pm 0.01)$ than oils not affected by TSR $(0.85 \pm 0.42$; Lillis and Selby, 2013). Therefore, it is likely that the higher Osi value in the Kora-1(B) resulted from alteration by TSR in the volcanic reservoir.

\subsubsection{Family 42 oils}

Thermal maturity models from a number of sites in the East Coast Basin indicate that significant oil generation from the Whangai Formation occurred from the Miocene to Pliocene 
in areas where there is significant Neogene sediment overburden (Field et al., 1997; Schlumberger, 2017). An oil generation date of $10 \mathrm{Ma}$ was therefore again used to calculate Osi and $\mathrm{Osg}$ values for the Family 42 oils and the Whangai Formation source rock, respectively.

The Osi values for the Family 42 oils from the Kaikoura (0.86) and Waitangi (0.79) oil seeps are more radiogenic than the Osg values of the Upper Calcareous member of the Whangai Formation (0.46-0.58; Fig. 5.4b). This member was analysed in this study because it has the highest source rock potential amongst the four members of the Whangai Formation (Section 2.2; Field et al., 1997; Hollis and Manzano-Kareah, 2005). The deeper Rakauroa member would likely exhibit more radiogenic Osg values given that it was deposited in the late Cretaceous when the seawater ${ }^{187} \mathrm{Os} /{ }^{188}$ Os was more radiogenic $(0.6-0.7)$ than in the Paleocene (0.3-0.4) during the deposition of the other members of the formation (Hollis and ManzanoKareah, 2005; Peucker-Ehrenbrink and Ravizza, 2012). However, this member lacks a proven oil-prone organofacies to have generated the Kaikoura and Waitangi oil seeps (Hollis and Manzano-Kareah, 2005; Sykes et al., 2012). Therefore, it seems unlikely that the Os in these oil seeps was sourced from the Whangai Formation, consistent with previous suggestions of the presence of an as yet unidentified Whangai-type marine source rock in the East Coast Basin (Sykes et al., 2012; Burton et al., 2019).

\subsection{Conclusions}

This study evaluated for the first time, Re-Os systematics and geochronology in crude oil sourced from terrestrial coals. In addition, this study explored the utility of the Os isotope fingerprinting tool to delineate the origin of two oil families whose source kerogens exhibit varying proportions of both marine and terrestrial organic matter. The average $\operatorname{Re}(0.31 \mathrm{ppb})$ and ${ }^{192} \mathrm{Os}(5.5 \mathrm{ppt})$ concentrations in the asphaltene fractions of the coaly-sourced Family 11 oils are relatively low, as are those reported for their potential coaly source rocks, the Rakopi and North Cape formations, and other fluvio-deltaic coals reported elsewhere. The large scatter in ${ }^{187} \mathrm{Re} /{ }^{188} \mathrm{Os}$ and ${ }^{187} \mathrm{Os} /{ }^{188} \mathrm{Os}$ ratios for these oils precludes Re-Os geochronology. This scatter is attributed to a large variation in Osi values inherited from heterogeneous coaly source rocks as well as a lengthy oil generation history. The Re-Os systematics of these crude oils are not considered to have been significantly affected by contact with formation waters and thus, largely reflect those derived from the source rocks. The Osi values for Family 11 oils overlap 
with Osg values for the late Cretaceous Rakopi and North Cape formations, consistent with previous suggestions that organic-rich intervals from these sedimentary units are likely to have sourced the oils. These results, therefore, demonstrate the potential application of Os isotope fingerprinting to trace oils derived from coaly source rocks.

The average Re and ${ }^{192}$ Os concentrations for the marine oils from Families 41 (3.2 ppb and $71.3 \mathrm{ppt}$, respectively) and 42 (2.2 ppb and $45.2 \mathrm{ppt}$, respectively) are much higher than those of the Family 11 terrestrial oils. The Osi values for the two Family 41 oils from Kora-1 well in the northern part of the Taranaki Basin are notably different, with the Kora-1(A) oil from the Eocene Tangaroa sandstones having an Osi value of 0.63 that is indistinguishable from Osg values (0.48-0.68) of its potential source rock, the Waipawa Formation. The Osi value for the Kora-1(B) oil is more radiogenic, however, which is attributed to likely disturbance of the ReOs systematics by TSR in the Miocene volcanic reservoir. In the East Coast Basin, the Osi of the Family 42 Kaikoura and Waitangi seeps suggest these oils are unlikely to have been derived from the Whangai Formation, consistent with the likely presence of another Cretaceous marine source with Whangai-type organofacies (Sykes et al., 2012; Burton et al., 2019). These findings highlight the usefulness of the ${ }^{187} \mathrm{Os} /{ }^{188}$ Os fingerprinting technique in distinguishing potential sources of oils with otherwise similar organic geochemical characteristics, even in complex settings. 


\section{CONCLUSIONS AND OUTLOOK}

This thesis provides the first in-depth research into Re-Os geochronology and isotope systematics of New Zealand's coaly-sourced and marine petroleum systems. Organic-rich sedimentary rocks studied in this thesis are dominated by terrestrial-sourced organic matter but were deposited in a range of environments including continental (fluvial), transitional-marginal marine (deltaic) and marine (shelf-slope and deep water). This allowed for an assessment of the impact of various aspects of these different environments on Re-Os systematics, and potential for geochronology and Os isotope tracing.

As the three main research chapters of this thesis are written in the format of scientific papers, each chapter has its own specific conclusions. The purpose of this chapter is to briefly highlight significant scientific outcomes of the research presented herein and draw together the common threads from the three main chapters. Areas of potential future research are also discussed.

\subsection{Re-Os systematics in terrestrial organic matter-dominated sediments}

The dominance of terrestrial organic matter (66 to $98 \%$ degraded woody phytoclasts) in the marine Waipawa Formation provided an opportunity to investigate whether organic matter type plays a role in Re and Os uptake. Both of these elements are enriched in the Waipawa Formation, with concentrations (22.5-85.9 ppb Re and 397.9-598.6 ppt Os) that are more than an order of magnitude higher than reported values for the UCC (0.2-2 ppb Re and 30-50 ppt Os). Strong positive correlations exist between TOC and $\mathrm{Re}\left(\mathrm{R}^{2}=0.91\right)$ and ${ }^{192} \mathrm{Os}\left(\mathrm{R}^{2}=0.73\right)$ in samples of this formation from the Orui-1A core, suggesting an uptake mechanism that is linked to the abundance of organic matter. Therefore, it appears that the presence of large proportion of terrestrial organic matter in organic-rich marine sediments may not significantly affect Re and Os uptake, supporting a previous argument that chelating precursors or fundamental binding sites responsible for the uptake of the elements are present in all types of organic matter (Cumming et al., 2012). In contrast, there are no relationships between TOC content and Re and ${ }^{192}$ Os concentrations across the full sample set of coals and coaly mudstones from the Rakopi, North Cape, Farewell and Mangahewa formations. Strong negative correlations exist between TOC and $\operatorname{Re}\left(\mathrm{R}^{2}=0.94\right)$ and ${ }^{192} \mathrm{Os}\left(\mathrm{R}^{2}=0.89\right)$ concentrations for the Mangahewa Formation sample subset. This is unexpected for organophilic elements like 
Re and Os, but it can be explained by either the leaching of these elements that occurs in raised mire coals when rainwater drains down through peats or stronger marine influence in coaly mudstones (low TOC, high Re and Os) relative to the raised mire coals (high TOC, low Re and Os). These results show that Re and Os enrichment in organic-rich sediments does not simply relate to their organophilic properties, but also to petrofacies and depositional conditions (in this case marine influence) of the sediments.

The $\operatorname{Re}(0.11-1.33 \mathrm{ppb})$ and Os (14.2-66.2 ppt) concentrations for the strongly marineinfluenced coaly sediments of the Mangahewa Formations are, however, up to two orders of magnitude lower than values reported for similarly marine-influenced coals from the Matewan coalbed in the USA (Tripathy et al., 2015). This suggests that marine transgressions into peatforming environments may not always result in significant enrichment of Re and Os in coals. The key difference between the Matewan and Mangehewa coals is that the Matewan coalbed is directly overlain by a marine shale, suggesting that such a juxtaposition of depositional environments may be required for enhanced Re and Os enrichment in coals, for example, through post-depositional percolation of Re and Os-rich seawater from the overlying marine shale. Future Re-Os case studies of coals overlain by marine shales may corroborate these suggestions.

The results from the marine Waipawa Formation are conflicting regarding whether depositional redox conditions can be linked to Re and Os uptake and fractionation. On one hand, TS content, which is an indicator of euxinic depositional conditions, exhibits positive correlations with the abundance of $\operatorname{Re}\left(\mathrm{R}^{2}=0.84\right)$ and ${ }^{192} \mathrm{Os}\left(\mathrm{R}^{2}=0.72\right)$, as well as ${ }^{187} \mathrm{Re} /{ }^{188} \mathrm{Os}$ ratio $\left(\mathrm{R}^{2}=0.66\right)$. On the other hand, other indicators of redox conditions such as HI and OI show no relationship with Re and Os concentrations and fractionation. The coals and coaly mudstones from the Rakopi, North Cape, Farewell and Mangahewa formations also exhibit Re and Os concentrations that appear to be independent of significant variation in redox conditions (based on $\mathrm{HI}$ and $\mathrm{OI}$ ). Combined, these results suggest that the uptake and fractionation of Re and Os in organic-rich sediments may not be solely attributed to the levels of oxygenation in the water column.

The importance of a slow sedimentation rate in enhanced Re and Os enrichment in organicrich sediments was also investigated in this study. The Waipawa Formation exhibits very high sedimentation rates (up to $\sim 10.6 \mathrm{~cm} / \mathrm{ky}$ ) compared to other typical marine sediments such as 
the underlying Whangai Formation $(\sim 1.1 \mathrm{~cm} / \mathrm{ky})$. It is, however, significantly enriched in $\mathrm{Re}$ and Os, with concentration levels that are much higher than those of the Whangai Formation. This suggests that a slow sedimentation rate may not play a significant role in Re and Os uptake.

The factors controlling fractionation of $\mathrm{Re}$ and Os were evaluated for Farewell Formation samples from the Kupe South-5 and -6 cores. The Kupe South-5 samples exhibit six times more spread in ${ }^{187} \mathrm{Re} /{ }^{188}$ Os ratios (126 units) than those from the Kupe South-6 (21 units). This significant variation was attributed to differences in stability and variability of the depositional environments of the two sample sets. The Kupe South-5 samples were deposited in low-lying swamps that were proximal to an active channel with fluctuating high and low energy clastic sedimentation (Flores et al., 1998). In addition, these samples comprise variable vitrain (wood) lenses and streaks, leaves and macerated plant fragments that may have provided variable chelating surfaces for complexation of Re and Os, leading to higher ${ }^{187} \mathrm{Re} /{ }^{188} \mathrm{Os}$ fractionation (Flores et al., 1998; Cumming et al., 2012). These characteristics contrast with those of the Kupe South-6 samples which lack coarse sediments and paleosols, and were deposited in a distal, more stable floodplain lake setting (Constantine, 2008). Such a stable depositional setting has previously been suggested to allow a more homogeneous uptake of Re and Os, leading to the lower spread in ${ }^{187} \mathrm{Re} /{ }^{188} \mathrm{Os}$ ratios (Cumming et al., 2012).

\subsection{Paleoenvironmental information from initial ${ }^{187} \mathrm{Os} /{ }^{188} \mathrm{Os}$}

This thesis defined and assessed factors controlling the evolution of the seawater Os curve for the early Paleogene. The Osi values for the Waipawa $(\sim 0.28)$ and Whangai $(\sim 0.36)$ formations provided additional constraints on the low-resolution marine Os record of the mid-late Paleocene. These were used together with literature ${ }^{187} \mathrm{Os} /{ }^{188} \mathrm{Os}$ values from organic-rich sediments spanning the period between 70 and $50 \mathrm{Ma}$ to show that seawater Os gradually became less radiogenic from the latest Cretaceous, reaching a minimum value in the earliest late Paleocene ( $\sim 59 \mathrm{Ma})$ during the deposition of Waipawa Formation, and then increased through the later Paleocene and into the early Eocene. This composite Os isotope record is correlated with global temperature $\left(\delta^{18} \mathrm{O}\right.$ and $\left.\mathrm{TEX}_{86}\right)$ and carbon isotope $\left(\delta^{13} \mathrm{C}\right)$ records from the middle Paleocene to early Eocene, which is inferred to reflect climate-modulated changes in continental weathering patterns. The Os isotope record for the early Paleogene, however, remains poorly resolved, especially for the period between $64 \mathrm{Ma}$ and $59 \mathrm{Ma}$. As such, 
expanding the limited dataset currently available for this time period to provide further insights into variations in seawater Os during the early Paleogene would be a valuable area to pursue for future work.

Variations in Osi of the late Cretaceous-Eocene coals and coaly mudstones from the Rakopi, North Cape, Farewell and Mangahewa formations provided an indication of the nature and source of Os in the fluvio-deltaic sediments. Samples from the Rakopi Formation display Osi values that are radiogenic $(0.8-1.2)$, which is expected for coals deposited in entirely terrestrial settings and which source Os from weathering of surrounding older continental rocks. In contrast, Osi values for samples from the progressively younger North Cape, Farewell and Mangahewa formations are all significantly less radiogenic (0.3-0.5). This was attributed to variable levels of marine influence, both open ocean and restricted basin, with variable resulting influence from the moderately radiogenic contemporaneous seawater and contributions of less radiogenic Os from nearby 78-72 Ma mafic extrusives. Erosion of these mafic extrusives is suggested to have lowered the ${ }^{187} \mathrm{Os} /{ }^{188} \mathrm{Os}$ of a series of complex waterways that characterised the southern part of the Taranaki Basin at $\sim 68$ Ma during the deposition of the North Cape Formation. These waterways were more likely to have been partially or fully restricted from the open ocean because the Osi values for the North Cape Formation samples $(0.31-0.46)$ are lower than that of seawater at the time of deposition $(\sim 0.50)$. The Osi values for samples from the Farewell and Mangahewa formations are very similar to those of seawater at the time of their deposition, suggesting that the Os in these samples was likely sourced from the marine environment. These results show that, like marine sediments, coaly rocks exhibit significant variations in the Os isotope composition that reflect depositional conditions, degree of water-connectivity with the open ocean, and sediment source, and hence provide useful insights into paleoenvironmental conditions.

\subsection{Re-Os geochronology of terrestrial organic matter-dominated sediments}

Application of the Re-Os geochronometer to the Waipawa Formation yields a first radiometric depositional age of $57.5 \pm 3.5 \mathrm{Ma}$ for this unit. This age is consistent with biostratigraphic age estimates (59.4-58.7 Ma; Hollis et al., 2014). The higher uncertainty in the Re-Os age is attributed to a small spread in ${ }^{187} \mathrm{Re} /{ }^{188} \mathrm{Os}$ ratios and a younger age. The Waipawa Formation 
and correlated organofacies have always been challenging to date through biostratigraphy due to the presence of unconformities, scarcity of age-diagnostic fossils, poor age control for dinoflagellate datums and lack of reliable paleomagnetic data. It has been difficult to establish whether all known occurrences of the Waipawa organofacies are coeval (Hollis et al., 2014). This study demonstrates the potential utility of the Re-Os geochronometer in radiometric dating of these occurrences and other similar terrestrial organic matter-dominated marine sediments. With the insights gained from the Taylor White and Orui-1A samples, carefully targetted sampling of Waipawa-type material in other Southwest Pacific basins for Re-Os geochronology may establish whether this material is coeval.

This thesis also evaluated Re-Os geochronometry in coals and coaly mudstones from the Farewell and Mangahewa formations. The ${ }^{187} \mathrm{Re} /{ }^{188} \mathrm{Os}$ vs ${ }^{187} \mathrm{Os} /{ }^{188} \mathrm{Os}$ relationship in the Farewell Formation samples exhibits significant scatter without any linear trend, corroborating the findings of the only other attempt at Re-Os geochronology of fluvio-deltaic sediments from the Soldogg Formation, Finnmark Platform (Goswami et al., 2018). These authors attributed the scatter in the Soldogg Formation sediments to heterogeneity in the Osi. In the present study, it is suggested that either very minimal heterogeneity in Osi values or large variations in ${ }^{187} \mathrm{Re} /{ }^{188} \mathrm{Os}$ values is required to produce reliable isochrons, but not necessarily both. The Farewell Formation samples lack both of these features, explaining the lack of an isochron fit. For the Mangahewa Formation samples, it appears that strong marine influence during their deposition improved isochroneity of the ${ }^{187} \mathrm{Re} /{ }^{188} \mathrm{Os}$ vs ${ }^{187} \mathrm{Os} /{ }^{188} \mathrm{Os}$ relationship, which yields an isochron with an age of $28 \pm 16 \mathrm{Ma}$. Although this age is highly imprecise, it is within uncertainty of the estimated biostratigraphic age (37 Ma) for these samples. A Monte Carlo simulation of the propagation of errors suggests that the imprecision in this Re-Os age largely $(69 \%)$ occurred due to analytical uncertainties, possibly as a result of low Re and Os concentrations in the samples and small amounts of rock powder $(0.2 \mathrm{~g})$ used for analysis. As with the Farewell Formation samples, a lack of spread in ${ }^{187} \mathrm{Re} /{ }^{188} \mathrm{Os}$ ratios is also a feature of the Mangahewa Formation samples that likely contributed further to the imprecise Re-Os age. Therefore, this study shows that where there is significant marine influence, Osi may be sufficiently homogeneous to support the development of a Re-Os isochron, although improvements in analytical precision are needed to fully utilise this for dating coals. Alternatively, further detailed research into processes controlling Re-Os fractionation in fluvio- 
deltaic sediments, building on this study's findings, may allow more effective targeting of samples that would be more suitable for Re-Os geochronology.

\subsection{Re and Os in oils generated from terrestrial organic matter-dominated sediments}

This thesis explored the potential of the Re-Os geochronometer and Os isotope tracer in coalysourced Family 11 oils and marine oils from Families 41 and 42. These oils are inferred to have been sourced from fluvio-deltaic coals and coaly mudstones of the Rakopi and North Cape formations (Family 11) and marine shales of the Waipawa (Family 41) and Whangai (Family 42) formations. The asphaltene fractions of the Family 11 oils record low average $\operatorname{Re}(0.31$ $\mathrm{ppb})$ and ${ }^{192} \mathrm{Os}(5.5 \mathrm{ppt})$ concentrations, which is consistent with derivation of the oils from terrestrial coals with similarly low concentrations of the elements. These low $\mathrm{Re}$ and Os concentrations coupled with limited asphaltene contents made precise measurements difficult, leading to large uncertainties in the Re-Os data. Despite the low concentrations, the Re-Os systematics of these crude oils are not considered to have been severely affected by contact with formation waters and thus, likely reflect those derived from the source rocks.

The ${ }^{187} \mathrm{Re} /{ }^{188} \mathrm{Os}$ and ${ }^{187} \mathrm{Os} /{ }^{188} \mathrm{Os}$ ratios in the asphaltene fractions of Family 11 oils are scattered on an isochron diagram without any linear relationship. This is attributed to a large variation in Osi values (0.47-1.14) inherited from the source rocks at the time of oil generation (estimated to be $10 \mathrm{Ma}$ ) and a small spread in ${ }^{187} \mathrm{Re} /{ }^{188} \mathrm{Os}$ ratios (275 units). The large variation in Osi values for these oils can be linked to the heterogeneous nature of the vertically extensive $(\sim 3000 \mathrm{~m})$ potential Rakopi and North Cape coaly source rocks and a lengthy (20 Myr) oil generation event. These Osi values closely overlap with the range of Osg values for the Rakopi and North Cape formations at $10 \mathrm{Ma}(0.38-1.26)$, consistent with these sedimentary units being the likely source of the oils. These results, therefore, demonstrate the potential utility of Os isotopes in tracing coaly-sourced oils but are not readily amenable to the Re-Os geochronometer due to the wide range in ${ }^{187} \mathrm{Os} /{ }^{188} \mathrm{Os}$ of potential source rocks combined with a complex and lengthy oil generation event. Future investigation of oils produced from less heterogeneous coal seams, and importantly, with more simple thermal maturation and migration history may show better potential for Re-Os geochronometry of coaly-sourced oils. 
The Family 41 oils containing both marine and terrestrial organic matter from two different reservoirs of the Kora-1 well in the Taranaki Basin [Kora-1(A) and Kora-1(B)] exhibit higher average $\operatorname{Re}(3.2 \mathrm{ppb})$ and ${ }^{192} \mathrm{Os}(71.3 \mathrm{ppt})$ concentrations compared to the coaly-sourced family 11 oils. These two oils show notably different Osi values, however. The Kora-1(A) oil from an Eocene sandstone reservoir exhibits an $\mathrm{Oss}_{\mathrm{i}}$ value (0.63) that is indistinguishable from $\mathrm{Osg}$ values $(0.48-0.68)$ of the inferred Waipawa Formation source rock. In contrast, the Osi value (0.92) for the Kora-1(B) oil from a Miocene volcanic reservoir is more radiogenic, which is attributed to likely modification by secondary processes in the reservoir such as thermochemical sulfate reduction (TSR).

The origin of Family 42 oils (Kaikoura and Waitangi) from New Zealand's East Coast Basin remain enigmatic. The Os isotopes show that the Kaikoura and Waitangi oils are unlikely to have originated from the late Cretaceous-Paleocene Whangai Formation, as they exhibit more radiogenic Osi values ( 0.86 and 0.79 , respectively) than the Osg values (0.46-0.58) of the Upper Calcareous member of the formation, which has the highest source rock potential amongst the four members of the formation (e.g., Hollis and Manzano-Kareah, 2005). These results support previous suggestions that the Whangai Formation lacks oil-prone organofacies to have sourced the numerous oil seeps in the East Coast Basin, and that a separate Cretaceous marine source rock may be present in the basin. Overall, this study demonstrates the usefulness and limitations of the Re-Os isotopic system in dating and tracing crude oils from different (fluvio-deltaic and marine) petroleum systems. 


\section{References}

Al-Hajeri, M.M., Al Saeed, M., Derks, J., Thomas, F., Thomas, H., Armin, K., Martin, N., Oliver, S., Nicky, T., Dietrich, W., Björn, W., Duplo, K., Ken, P., 2009. Basin and petroleum system modeling. Oilfield Review, 21: 14-29.

Allègre, C.J., Luck, J.M., 1980. Osmium isotopes as petrogenetic and geological tracers. Earth and Planetary Science Letters, 48: 148-154.

Andreasen, R., Sharma, M., 2009. Fractionation and mixing in a thermal ionization mass spectrometer source: Implications and limitations for high-precision $\mathrm{Nd}$ isotope analyses. International Journal of Mass Spectrometry, 285: 49-57.

ASTM International, 2018. ASTM D4239-18e1, standard test method for sulfur in the analysis sample of coal and coke using high-temperature tube furnace combustion, ASTM International, West Conshohocken, Pennsylvania, 8 pp.

Baioumy, H.M., Eglinton, L.B., Peucker-Ehrenbrink, B., 2011. Rhenium-osmium isotope and platinum group element systematics of marine vs. non-marine organic-rich sediments and coals from Egypt. Chemical Geology, 285: 70-81.

Ballance, P.F., Spörli, K.B., 1979. Northland Allochthon. Journal of the Royal Society of New Zealand, 9: 259-275.

Banerjee, I., Goodarzi, F., 1990. Paleoenvironment and sulfur-boron contents of the Mannville (lower Cretaceous) coals of southern Alberta, Canada. Sedimentary Geology, 67: 297310.

Barnes, S.-J., Ripley, E.M., 2016. Highly siderophile and strongly chalcophile elements in magmatic ore deposits. Reviews in Mineralogy and Geochemistry, 81: 725-774.

Barnet, J.S.K., Littler, K., Westerhold, T., Kroon, D., Leng, M.J., Bailey, I., Röhl, U., Zachos, J.C., 2019. A High-fidelity benthic stable isotope record of late Cretaceous-early Eocene climate change and carbon-cycling. Paleoceanography and Paleoclimatology, 34: 672-691.

Barre, A., Prinzhofer, A., Allegre, C., 1995. Osmium isotopes in the organic matter of crude oil and asphaltenes, Terra Abstracts, $199 \mathrm{pp}$.

Barron, E.J., Peterson, W.H., 1991. The Cenozoic ocean circulation based on ocean General Circulation Model results. Palaeogeography, Palaeoclimatology, Palaeoecology, 83: 128.

Batenburg, S.J., Voigt, S., Friedrich, O., Osborne, A.H., Bornemann, A., Klein, T., Pérez-Díaz, L., Frank, M., 2018. Major intensification of Atlantic overturning circulation at the onset of Paleogene greenhouse warmth. Nature Communications, 9: 4954.

Beck, R.A., Burbank, D.W., Sercombe, W.J., Olson, T.L., Khan, A.M., 1995. Organic carbon exhumation and global warming during the early Himalayan collision. Geology, 23: 387-390.

Becker, J.S., 2012. Chapter 13 - Inorganic Mass Spectrometry of Radionuclides. In: L'Annunziata, M.F. (Ed.), Handbook of Radioactivity Analysis (Third Edition). Academic Press, Amsterdam, pp. 833-870.

Bertoni, M.E., Rooney, A.D., Selby, D., Alkmim, F.F., Le Heron, D.P., 2014. Neoproterozoic Re-Os systematics of organic-rich rocks in the São Francisco Basin, Brazil and implications for hydrocarbon exploration. Precambrian Research, 255: 355-366.

Bijl, P.K., Schouten, S., Sluijs, A., Reichart, G.-J., Zachos, J.C., Brinkhuis, H., 2009. Early Palaeogene temperature evolution of the southwest Pacific Ocean. Nature, 461: 776779. 
Birck, J.L., 2001. The precision and sensitivity of thermal ionisation mass spectrometry (TIMS): An overview of the present status. Geostandards Newsletter, 25: 253-259.

Birck, J.L., Barman, M.R., Capmas, F., 1997. Re-Os isotopic measurements at the femtomole level in natural samples. Geostandards Newsletter, 21: 19-27.

Bjorøy, M., Williams, J.A., Dolcater, D.L., Kemp, M.K., Winters, J.C., 1996. Maturity assessment and characterization of Big Horn Basin Palaeozoic oils. Marine and Petroleum Geology, 13: 3-23.

Bland, K.J., Strogen, D.P., Morgans, H.E.G., Ventura, G.T., 2014. Record of section descriptions and sample collection from the Tahuokaretu and Taylor White sections, southern Hawke's Bay, 1-3 May 2014, for micropaleontological and source rock analyses. GNS Science internal report 2014/03, 55 pp.

Bohacs, K., Suter, J., 1997. Sequence stratigraphic distribution of coaly rocks: Fundamental controls and paralic examples. American Association of Petroleum Geologists Bulletin, 81: 1612-1639.

Brett, W., 2005. Hydrocarbon potential of the Kora structure, PEP 38485. Ministry of Economic Development, New Zealand, unpublished Petroleum Report 3346, 32 pp.

Browne, G.H., Kennedy, E.M., Constable, R.M., Raine, J.I., Crouch, E.M., Sykes, R., 2008. An outcrop-based study of the economically significant Late Cretaceous Rakopi Formation, northwest Nelson, Taranaki Basin, New Zealand. New Zealand Journal of Geology and Geophysics, 51: 295-315.

Burton, K.W., Bourdon, B., Birck, J.-L., Allègre, C.J., Hein, J.R., 1999. Osmium isotope variations in the oceans recorded by Fe-Mn crusts. Earth and Planetary Science Letters, 171: 185-197.

Burton, K.W., Gannoun, A., Birck, J.-L., Allègre, C.J., Schiano, P., Clocchiatti, R., Alard, O., 2002. The compatibility of rhenium and osmium in natural olivine and their behaviour during mantle melting and basalt genesis. Earth and Planetary Science Letters, 198: 6376.

Burton, Z.F.M., Moldowan, J.M., Magoon, L.B., Sykes, R., Graham, S.A., 2019. Correction to: Interpretation of source rock depositional environment and age from seep oil, east coast of New Zealand. International Journal of Earth Sciences, 108: 1093-1093.

Busch, K., 2011. Detecting Ions in Mass Spectrometers with the Faraday Cup. Spectroscopy, 26: $12-18$.

Calkins, W.H., 1994. The chemical forms of sulfur in coal: a review. Fuel, 73: 475-484.

Chou, C.-L., 2012. Sulfur in coals: A review of geochemistry and origins. International Journal of Coal Geology, 100: 1-13.

Clayton, C., 2011. 3D Basin modelling of PEP 38485, offshore Taranaki Basin. Ministry of Economic Development, New Zealand, unpublished Petroleum Report 4313, 110 pp.

Cohen, A.S., 2004. The rhenium-osmium isotope system: applications to geochronological and palaeoenvironmental problems. Journal of the Geological Society, 161: 729-734.

Cohen, A.S., Coe, A.L., 2002. New geochemical evidence for the onset of volcanism in the Central Atlantic magmatic province and environmental change at the Triassic-Jurassic boundary. Geology, 30: 267-27.

Cohen, A.S., Coe, A.L., 2007. The impact of the Central Atlantic Magmatic Province on climate and on the Sr- and Os-isotope evolution of seawater. Palaeogeography, Palaeoclimatology, Palaeoecology, 244: 374-390.

Cohen, A.S., Coe, A.L., Bartlett, J.M., Hawkesworth, C.J., 1999. Precise Re-Os ages of organic-rich mudrocks and the Os isotope composition of Jurassic seawater. Earth and Planetary Science Letters, 167: 159-173. 
Cohen, A.S., Waters, F.G., 1996. Separation of osmium from geological materials by solvent extraction for analysis by thermal ionisation mass spectrometry. Analytica Chimica Acta, 332: 269-275.

Collinson, J., 2005. Sedimentary processes | Depositional Sedimentary Structures. In: Selley, R.C., Cocks, L.R.M., Plimer, I.R. (Eds.), Encyclopedia of Geology. Elsevier, Oxford, pp. 593-602.

Colodner, D., Sachs, J., Ravizza, G., Turekian, K.K., Edmond, J., Boyle, E., 1993. The geochemical cycle of rhenium: a reconnaissance. Earth and Planetary Science Letters, 117: 205-221.

Constantine, A., 2008. Kupe South-6 well completion report. Ministry of Economic Development New Zealand, unpublished Petroleum Report 4019, 2624 pp.

Cooper, R.A., Agterberg, F.P., Alloway, B.V., Beu, A.G., Campbell, H.J., Crampton, J.S., Crouch, E.M., Crundwell, M.P., Graham, I.J., Hollis, C.J., Jones, C.M., Kamp, P.J.J., Mildenhall, D.C., Morgans, H.E.G., Naish, T.R., Raine, J.I., Roncaglia, L., Sadler, P.M., Schiøler, P., Scott, G.H., Strong, C.P., Wilson, G.J., Wilson, G.S., 2004. The New Zealand Geological Timescale, Institute of Geological and Nuclear Sciences Monograph 22. Institute of Geological and Nuclear Sciences, Lower Hutt, New Zealand, $284 \mathrm{pp}$.

Corfield, R.M., Cartlidge, J.E., 1992. Oceanographic and climatic implications of the Palaeocene carbon isotope maximum. Terra Nova, 4: 443-455.

Correia, C., Frick, L., Petronilho, L., Celso, C., Tassinari, C., Sato, K., 2007. The Re-Os isotopic system: geochemistry and methodology at the Geochronological Research Center (CPGeo) of the University of São Paulo, Brazil. Geologia USP. Série Científica, 7: $45-56$.

Corrick, A.J., Selby, D., McKirdy, D.M., Hall, P.A., Gong, S., Trefry, C., Ross, A.S., 2019. Remotely constraining the temporal evolution of offshore oil systems. Scientific Reports, 9: 1327.

Creaser, R., Szatmari, P., Milani, E.J., 2008. Extending Re-Os shale geochronology to lacustrine depositional systems: a case study from the major hydrocarbon source rocks of the Brazilian Mesozoic marginal basins, Proceedings of the 33rd International Geological Congress, Oslo. Abstract.

Creaser, R.A., Papanastassiou, D.A., Wasserburg, G.J., 1991. Negative thermal ion mass spectrometry of osmium, rhenium, and iridium. Geochimica et Cosmochimica Acta, 55: $391-401$.

Creaser, R.A., Sannigrahi, P., Chacko, T., Selby, D., 2002. Further evaluation of the Re-Os geochronometer in organic-rich sedimentary rocks: a test of hydrocarbon maturation effects in the Exshaw Formation, Western Canada Sedimentary Basin. Geochimica et Cosmochimica Acta, 66: 3441-3452.

Crouch, E.M., Raine, J.I., 2012. Palynological study of the late Paleocene-early Eocene Kapuni Group section from four wells in the Kupe region, Taranaki Basin. GNS Science Consultancy Report 2012/125, 24 pp.

Crouch, E.M., Willumsen, P.S., Kulhanek, D.K., Gibbs, S.J., 2014. A revised Paleocene (Teurian) dinoflagellate cyst zonation from eastern New Zealand. Review of Palaeobotany and Palynology, 202: 47-79.

Crusius, J., Calvert, S., Pedersen, T., Sage, D., 1996. Rhenium and molybdenum enrichments in sediments as indicators of oxic, suboxic and sulfidic conditions of deposition. Earth and Planetary Science Letters, 145: 65-78. 
Crusius, J., Thomson, J., 2000. Comparative behavior of authigenic Re, U, and Mo during reoxidation and subsequent long-term burial in marine sediments. Geochimica et Cosmochimica Acta, 64: 2233-2242.

Cumming, V.M., Selby, D., Lillis, P.G., 2012. Re-Os geochronology of the lacustrine Green River Formation: Insights into direct depositional dating of lacustrine successions, ReOs systematics and paleocontinental weathering. Earth and Planetary Science Letters, 359-360: 194-205.

Cumming, V.M., Selby, D., Lillis, P.G., Lewan, M.D., 2014. Re-Os geochronology and Os isotope fingerprinting of petroleum sourced from a Type I lacustrine kerogen: Insights from the natural Green River petroleum system in the Uinta Basin and hydrous pyrolysis experiments. Geochimica et Cosmochimica Acta, 138: 32-56.

Dalai, T.K., Ravizza, G.E., Peucker-Ehrenbrink, B., 2006. The Late Eocene ${ }^{187} \mathrm{Os} /{ }^{188} \mathrm{Os}$ excursion: Chemostratigraphy, cosmic dust flux and the Early Oligocene glaciation. Earth and Planetary Science Letters, 241: 477-492.

Dalai, T.K., Suzuki, K., Minagawa, M., Nozaki, Y., 2005. Variations in seawater osmium isotope composition since the last glacial maximum: A case study from the Japan Sea. Chemical Geology, 220: 303-314.

Dauphas, N., Reisberg, L., Marty, B., 2002. An alternative explanation for the distribution of highly siderophile elements in the Earth. Geochemical Journal, 36: 409-419.

Day, J.M.D., Brandon, A.D., Walker, R.J., 2016. Highly siderophile elements in Earth, Mars, the Moon, and Asteroids. Reviews in Mineralogy and Geochemistry, 81: 161-238.

De Lena, L.F., Taylor, D., Guex, J., Bartolini, A., Adatte, T., van Acken, D., Spangenberg, J.E., Samankassou, E., Vennemann, T., Schaltegger, U., 2019. The driving mechanisms of the carbon cycle perturbations in the late Pliensbachian (Early Jurassic). Scientific Reports, 9: 18430.

Dickin, A.P., 2018. Radiogenic Isotope Geology. Cambridge University Press, Cambridge, 501 pp.

Dickson, A.J., Cohen, A.S., Coe, A.L., Davies, M., Shcherbinina, E.A., Gavrilov, Y.O., 2015. Evidence for weathering and volcanism during the PETM from Arctic Ocean and PeriTethys osmium isotope records. Palaeogeography, Palaeoclimatology, Palaeoecology, 438: 300-307.

Didyk, B.M., Simoneit, B.R.T., Brassell, S.C., Eglinton, G., 1978. Organic geochemical indicators of palaeoenvironmental conditions of sedimentation. Nature, 272: 216-222.

Diessel, C.F.K., 1992. Coal-Bearing Depositional Systems. Springer-Verlag, Berlin, Heidelberg, $721 \mathrm{pp}$.

DiMarzio, J.M., Georgiev, S.V., Stein, H.J., Hannah, J.L., 2018. Residency of rhenium and osmium in a heavy crude oil. Geochimica et Cosmochimica Acta, 220: 180-200.

Du Vivier, A.D.C., Selby, D., Condon, D.J., Takashima, R., Nishi, H., 2015. Pacific ${ }^{187}$ Os $/{ }^{188}$ Os isotope chemistry and $\mathrm{U}-\mathrm{Pb}$ geochronology: Synchroneity of global Os isotope change across OAE 2. Earth and Planetary Science Letters, 428: 204-216.

Du Vivier, A.D.C., Selby, D., Sageman, B.B., Jarvis, I., Gröcke, D.R., Voigt, S., 2014. Marine ${ }^{187} \mathrm{Os} /{ }^{188} \mathrm{Os}$ isotope stratigraphy reveals the interaction of volcanism and ocean circulation during Oceanic Anoxic Event 2. Earth and Planetary Science Letters, 389: 23-33.

Dubin, A., Peucker-Ehrenbrink, B., 2015. The importance of organic-rich shales to the geochemical cycles of rhenium and osmium. Chemical Geology, 403: 111-120.

Edbrooke, S.W., Sykes, R., Pocknall, D.T., 1994. Geology of the Waikato Coal Measures, Waikato Coal Region, New Zealand, Institute of Geological and Nuclear Sciences 
monograph 6. Institute of Geological and Nuclear Sciences, Lower Hutt, New Zealand, $236 \mathrm{pp}$.

Energy and Building Trends, 2017. Energy in New Zealand 2017. Ministry of Business, Innovation and Employment, Wellington, New Zealand, $47 \mathrm{pp}$.

Espitalié, J., Madec, M., Tissot, B., Mennig, J.J., Leplat, P., 1977. Source rock characterization method for petroleum exploration. Offshore Technology Conference, Houston, Texas, $6 \mathrm{pp}$.

Esser, B.K., Turekian, K.K., 1993. The osmium isotopic composition of the continental crust. Geochimica et Cosmochimica Acta, 57: 3093-3104.

Fehn, U., Teng, R., Elmore, D., Kubik, P.W., 1986. Isotopic composition of osmium in terrestrial samples determined by accelerator mass spectrometry. Nature, 323: 707-710.

Field, B.D., Naeher, S., Clowes, C.D., Shepherd, C.L., Hollis, C.J., Sykes, R., Ventura, G.T., Pascher, K.M., Griffin, A.G., 2018. Depositional influences on the petroleum potential of the Waipawa Formation in the Orui-1A drillhole, Wairarapa. GNS Science report 2017/49, 75 pp.

Field, B.D., Uruski, C.I., Beu, A.G., Browne, G.H., Crampton, J.S., Funnell, R.H., Killops, S.D., Laird, M., Mazengarb, C., Morgans, H.E.G., Rait, G.J., Smale, D., Strong, C.P., 1997. Cretaceous-Cenozoic geology and petroleum systems of the East Coast region, New Zealand, Monograph 19, 7 enclosures. Institute of Geological \& Nuclear Sciences Limited, Lower Hutt, New Zealand, 301 pp.

Finlay, A.J., Selby, D., Gröcke, D.R., 2010. Tracking the Hirnantian glaciation using Os isotopes. Earth and Planetary Science Letters, 293: 339-348.

Finlay, A.J., Selby, D., Osborne, M.J., 2011. Re-Os geochronology and fingerprinting of United Kingdom Atlantic margin oil: Temporal implications for regional petroleum systems. Geology, 39: 475-478.

Finlay, A.J., Selby, D., Osborne, M.J., 2012. Petroleum source rock identification of United Kingdom Atlantic Margin oil fields and the Western Canadian Oil Sands using Platinum, Palladium, Osmium and Rhenium: Implications for global petroleum systems. Earth and Planetary Science Letters, 313-314: 95-104.

Flores, R.M., 2004. Coal buildup in tide-influenced coastal plains in the Eocene Kapuni Group, Taranaki Basin, New Zealand. In: Pashin, J.C., Gastaldo, R.A. (Eds.), Sequence Stratigraphy, Paleoclimate, and Tectonics of Coal-Bearing Strata. AAPG Studies in Geology, 51: 45-70.

Flores, R.M., Browne, G.H., Sykes, R., 1998. Lithofacies, depositional setting and reservoir characteristics of the Farewell Formation, Kupe South Field, Taranaki Basin, New Zealand, 1998 New Zealand Petroleum Conference Proceedings. Ministry of Commerce, Crown Minerals, Ministry of Commerce, Wellington, New Zealand, pp. 499-512.

Flores, R.M., Sykes, R., 1996. Depositional controls on coal distribution and quality in the Eocene Brunner Coal Measures, Buller Coalfield, South Island, New Zealand. International Journal of Coal Geology, 29: 291-336.

Funnell, R., Stagpoole, V.M., Nicol, A., Killops, S., Reyes, A.G., Darby, D., 2001. Migration of oil and gas into the Maui Field, Taranaki Basin, New Zealand, Eastern Australia Basins Symposium, pp. 121-128.

Funnell, R., Stagpoole, V.M., Nicol, A., McCormack, N., Reyes, A.G., 2004. Petroleum generation and implications for migration: a Maui Field charge study, Taranaki Basin, 2004 New Zealand Petroleum Conference Proceedings. Ministry of Economic Development, Wellington, 9 pp. 
Gannoun, A., Burton, K.W., 2014. High precision osmium elemental and isotope measurements of North Atlantic seawater. Journal of Analytical Atomic Spectrometry, 29: 2330-2342.

Gayer, R.A., Rose, M., Dehmer, J., Shao, L.Y., 1999. Impact of sulphur and trace element geochemistry on the utilization of a marine-influenced coal — case study from the South Wales Variscan foreland basin. International Journal of Coal Geology, 40: 151-174.

Ge, X., Shen, C., Selby, D., Deng, D., Mei, L., 2016. Apatite fission-track and Re-Os geochronology of the Xuefeng uplift, China: Temporal implications for dry gas associated hydrocarbon systems. Geology, 44: 491-494.

Ge, X., Shen, C., Selby, D., Wang, J., Ma, L., Ruan, X., Hu, S., Mei, L., 2018. Petroleumgeneration timing and source in the northern Longmen Shan thrust belt, Southwest China: Implications for multiple oil-generation episodes and sources. American Association of Petroleum Geologists Bulletin, 102: 913-938.

Geboy, N.J., Tripathy, G.R., Ruppert, L.F., Eble, C.F., Blake, B.M., Hannah, J.L., Stein, H.J., 2015. Re-Os age for the Lower-Middle Pennsylvanian Boundary and comparison with associated palynoflora. International Journal of Coal Geology, 140: 23-30.

Georgiev, S., Stein, H.J., Hannah, J.L., Bingen, B., Weiss, H.M., Piasecki, S., 2011. Hot acidic Late Permian seas stifle life in record time. Earth and Planetary Science Letters, 310 : 389-400.

Georgiev, S., Stein, H.J., Hannah, J.L., Weiss, H.M., Bingen, B., Xu, G., Rein, E., Hatl, V., Lseth, H., Nali, M., Piasecki, S., 2012. Chemical signals for oxidative weathering predict Re-Os isochroneity in black shales, East Greenland. Chemical Geology, 324325: 108-121.

Georgiev, S.V., Stein, H.J., Hannah, J.L., Galimberti, R., Nali, M., Yang, G., Zimmerman, A., 2016. Re-Os dating of maltenes and asphaltenes within single samples of crude oil. Geochimica et Cosmochimica Acta, 179: 53-75.

Georgiev, S.V., Stein, H.J., Hannah, J.L., Xu, G., Bingen, B., Weiss, H.M., 2017. Timing, duration, and causes for Late Jurassic-Early Cretaceous anoxia in the Barents Sea. Earth and Planetary Science Letters, 461: 151-162.

Georgiev, S.V., Stein, H.J., Hannah, J.L., Yang, G., Markey, R.J., Dons, C.E., Pedersen, J.H., di Primio, R., 2019. Comprehensive evolution of a petroleum system in absolute time: The example of Brynhild, Norwegian North Sea. Chemical Geology, 522: 260-282.

Gill, R., 1997. Modern Analytical Geochemistry - An Introduction to Quantitative Chemical Analysis Techniques for Earth, Environmental and Materials Scientists. Taylor \& Francis, London, $342 \mathrm{pp}$.

Goldstein, T.P., Aizenshtat, Z., 1994. Thermochemical sulfate reduction a review. Journal of Thermal Analysis, 42: 241-290.

Goswami, V., Hannah, J.L., Stein, H.J., 2018. Why terrestrial coals cannot be dated using the Re-Os geochronometer: Evidence from the Finnmark Platform, southern Barents Sea and the Fire Clay coal horizon, Central Appalachian Basin. International Journal of Coal Geology, 188: 121-135.

Gradstein, F.M., Ogg, J.G., Schmitz, M., Ogg, G., 2012. The Geologic Time Scale 2012, 2. Elsevier, 229 pp.

Gramlich, J.W., Murphy, T.J., Garner, E.L., Shields, W.R., 1973. Absolute isotopic abundance ratio and atomic weight of a reference sample of rhenium. Journal of Research of the National Bureau of Standards A, 77A: 691-698.

Haq, B.U., 1981. Paleogene Paleoceanography: Early Cenozoic Oceans Revisited. Oceanologica Acta, Special issue (0399-1784). 
Harris, N.B., Mnich, C.A., Selby, D., Korn, D., 2013. Minor and trace element and Re-Os chemistry of the Upper Devonian Woodford Shale, Permian Basin, west Texas: Insights into metal abundance and basin processes. Chemical Geology, 356: 76-93.

Harrison, A., Reld, E., Smith, N., 2013. PEP 51906 Seismic Interpretation Report. Ministry of Business, Innovation and Employment, New Zealand, unpublished Petroleum Report 4664, 79 pp.

Hart, S.R., Zindler, A., 1989. Isotope fractionation laws: a test using calcium. International Journal of Mass Spectrometry and Ion Processes, 89: 287-301.

Harvey, J., Warren, J.M., Shirey, S.B., 2016. Mantle sulfides and their role in Re-Os and Pb isotope geochronology. Reviews in Mineralogy and Geochemistry, 81: 579-649.

Hattori, K., 1996. Occurrence and origin of sulfide and sulfate in the 1991 Mount Pinatubo eruption products. In: C.G. Newhall, C.G., Punongbayan, R.S. (Eds.), Fire and Mud: Eruptions and Lahars of Mount Pinatubo, Philipines. University of Washington Press, pp. 807-824.

Hattori, K., Menagh, D., Cole, T., 1998. Low blank isotope ratio measurements of rhenium, osmium, and platinum using tantalum filaments with negative thermal ionization mass spectrometry. Analytical Chemistry, 70: 4100-4103.

Herr, W., Hintenberger, H., Voshage, H., 1954. Half-Life of rhenium. Physical Review, 95: $1691-1691$.

Herr, W., Wolfle, R., Eberhardt, P., Kopp, E., 1967. Development and recent applications of the $\mathrm{Re} / \mathrm{Os}$ dating method, Radioactive dating and methods of low-level counting. International Atomic Energy Agency, Monaco, pp. 499-508.

Hertogen, J., Janssens, M.J., Palme, H., 1980. Trace elements in ocean ridge basalt glasses: implications for fractionations during mantle evolution and petrogenesis. Geochimica et Cosmochimica Acta, 44: 2125-2143.

Higgs, K., King, P., Sykes, R., Crouch, E., Browne, G., Raine, I., Strogen, D., Brathwaite, B., Chagué-Goff, C., Palmer, J., 2009. Middle to Late Eocene Stratigraphy, Taranaki Basin, New Zealand. A catalogue integrating biostratigraphy, sedimentology, geochemistry and log data over the NE-SW trending Mangahewa Formation fairway. GNS Science Science Report 2009/30, CD.

Higgs, K.E., Arnot, M.J., Browne, G.H., Kennedy, E.M., 2010. Reservoir potential of Late Cretaceous terrestrial to shallow marine sandstones, Taranaki Basin, New Zealand. Marine and Petroleum Geology, 27: 1849-1871.

Higgs, K.E., King, P., Sykes, R., Crouch, E., Browne, G., Chagué-Goff, C., Palmer, J., Raine, I., 2006. Mangahewa Project: A multidisciplinary study of the mid-late Eocene succession, onshore Taranaki Basin. Phase one: Catalogue of stratigraphy, depositional environment and petrology along a NW-SE trending transect line. GNS Science Report 2006/18, CD.

Higgs, K.E., Raine, J.I., Baur, J.R., Sykes, R., King, P.R., Browne, G.H., Crouch, E., 2012. Depositional age, facies, and cyclicity within the Mangahewa reservoir fairway, Middle to Late Eocene, Taranaki Basin. GNS Science Report 2011/47, 108 pp.

Hines, B.R., Gazley, M.F., Collins, K.S., Bland, K.J., Crampton, J.S., Ventura, G.T., 2019. Chemostratigraphic resolution of widespread reducing conditions in the southwest Pacific Ocean during the Late Paleocene. Chemical Geology, 504: 236-252.

Hirt, B., Tilton, G.R., Herr, W., Hoffmeister, W., 1963. The half-life of ${ }^{187}$ Re. In: Geiss, J., Goldberg, E. (Eds.), Earth Science and Meteoritics. North Holland, Amsterdam, pp. 273-280. 
Hnatyshin, D., Rostron, B., Creaser, R., 2019. Re-Os isotope systematics from deep water collected from the Western Canada Sedmentary Basin (WCSB), Goldschmidt, Barcelona, Abstract.

Hollis, C.J., Field, B.D., Crouch, E.M., Sykes, R., 2006. How good a source rock is the Waipawa (black shale) Formation beyond the East Coast Basin? An outcrop-based case study from Northland, 2006 New Zealand Petroleum Conference Proceedings, Auckland, New Zealand, 8 pp.

Hollis, C.J., Manzano-Kareah, K., 2005. Source rock potential of the East Coast Basin. Ministry of Economic Development New Zealand, unpublished Petroleum Report 3179, $158 \mathrm{pp}$.

Hollis, C.J., Tayler, M.J.S., Andrew, B., Taylor, K.W., Lurcock, P., Bijl, P.K., Kulhanek, D.K., Crouch, E.M., Nelson, C.S., Pancost, R.D., Huber, M., Wilson, G.S., Ventura, G.T., Crampton, J.S., Schiøler, P., Phillips, A., 2014. Organic-rich sedimentation in the South Pacific Ocean associated with Late Paleocene climatic cooling. Earth-Science Reviews, 134: 81-97.

Hollis, C.J., Taylor, K.W.R., Handley, L., Pancost, R.D., Huber, M., Creech, J.B., Hines, B.R., Crouch, E.M., Morgans, H.E.G., Crampton, J.S., Gibbs, S., Pearson, P.N., Zachos, J.C., 2012. Early Paleogene temperature history of the Southwest Pacific Ocean: Reconciling proxies and models. Earth and Planetary Science Letters, 349-350: 53-66.

Hunt, J.M., 1995. Petroleum Geochemistry and Geology. W. H. Freeman and Company, New York, $743 \mathrm{pp}$.

Hurtig, N.C., Georgiev, S.V., Stein, H.J., Hannah, J.L., 2019. Re-Os systematics in petroleum during water-oil interaction: The effects of oil chemistry. Geochimica et Cosmochimica Acta, 247: 142-161.

Hurtig, N.C., Georgiev, S.V., Zimmerman, A., Yang, G., Goswami, V., Hannah, J.L., Stein, H.J., 2020. Re-Os geochronology for the NIST RM 8505 crude oil: The importance of analytical protocol and uncertainty. Chemical Geology, 539: 119381.

Ibach, L.E.J., 1982. Relationship between sedimentation rate and total organic carbon content in ancient marine sediments. American Association of Petroleum Geologists Bulletin, 66: $170-188$.

Ingall, E.D., Cappellen, P.V., 1990. Relation between sedimentation rate and burial of organic phosphorus and organic carbon in marine sediments. Geochimica et Cosmochimica Acta, 54: 373-386.

Jaffe, L.A., Peucker-Ehrenbrink, B., Petsch, S.T., 2002. Mobility of rhenium, platinum group elements and organic carbon during black shale weathering. Earth and Planetary Science Letters, 198: 339-353.

Jones, M.M., Ibarra, D.E., Gao, Y., Sageman, B.B., Selby, D., Chamberlain, C.P., Graham, S.A., 2018. Evaluating Late Cretaceous OAEs and the influence of marine incursions on organic carbon burial in an expansive East Asian paleo-lake. Earth and Planetary Science Letters, 484: 41-52.

Kendall, B., Creaser, R.A., Gordon, G.W., Anbar, A.D., 2009a. Re-Os and Mo isotope systematics of black shales from the Middle Proterozoic Velkerri and Wollogorang Formations, McArthur Basin, northern Australia. Geochimica et Cosmochimica Acta, 73: 2534-2558.

Kendall, B., Creaser, R.A., Selby, D., 2006. Re-Os geochronology of postglacial black shales in Australia: Constraints on the timing of "Sturtian" glaciation. Geology, 34: 729-732.

Kendall, B., Creaser, R.A., Selby, D., 2009b. ${ }^{187}$ Re $-{ }^{187}$ Os geochronology of Precambrian organic-rich sedimentary rocks. The Geological Society, London, Special Publications, 326: 85-107. 
Kendall, B.S., Creaser, R.A., Ross, G.M., Selby, D., 2004. Constraints on the timing of Marinoan "Snowball Earth" glaciation by ${ }^{187} \mathrm{Re}^{-187}$ Os dating of a Neoproterozoic, postglacial black shale in Western Canada. Earth and Planetary Science Letters, 222: 729740.

Killops, S.D., Cook, R.A., Sykes, R., Boudou, J.P., 1997. Petroleum potential and oil-source correlation in the Great South and Canterbury Basins. New Zealand Journal of Geology and Geophysics, 40: 405-423.

Killops, S.D., Hollis, C.J., Morgans, H.E.G., Sutherland, R., Field, B.D., Leckie, D.A., 2000. Paleoceanographic significance of Late Paleocene dysaerobia at the shelf/slope break around New Zealand. Palaeogeography, Palaeoclimatology, Palaeoecology, 156: 5170.

Killops, S.D., Raine, J.I., Woolhouse, A.D., Weston, R.J., 1995. Chemostratigraphic evidence of higher-plant evolution in the Taranaki Basin, New Zealand. Organic Geochemistry, 23: 429-445.

Killops, S.D., Woolhouse, A.D., Weston, R.J., Cook, R.A., 1994. A geochemical appraisal of oil generation in the Taranaki Basin, New Zealand. American Association of Petroleum Geologists Bulletin, 78: 1560-1584.

King, P.R., 2000. New Zealand's changing configuration in the last 100 million years; plate tectonics, basin development, and depositional setting, 2000 New Zealand Petroleum Conference Proceedings, Crown Minerals, Ministry of Commerce Wellington, New Zealand, pp. 131-145.

King, P.R., Thrasher, G.P., 1996. Cretaceous-Cenozoic geology and petroleum systems of the Taranaki Basin, New Zealand. Institute of Geological \& Nuclear Sciences Ltd., 234 pp.

Klemm, V., Levasseur, S., Frank, M., Hein, J.R., Halliday, A.N., 2005. Osmium isotope stratigraphy of a marine ferromanganese crust. Earth and Planetary Science Letters, 238: 42-48.

Kroeger, K., Funnell, R., Arnot, M., S, B., M, H., Sahoo, T., H, Z., 2016. Re-assessment of maturity and charge in southern Taranaki Basin (New Zealand) using integrated 3D basin modelling, AAPG/SEG International Conference \& Exhibition 2015. Eastern Australian Basins Symposium, Melbourne, Australia, pp. 217-230.

Kulhanek, D., Crouch, E., J. S. Tayler, M., Hollis, C., 2015. Paleocene calcareous nannofossils from East Coast, New Zealand: biostratigraphy and palaeoecology. Journal of Nannoplankton Research, 35: 155-176.

Kurtz, A.C., Kump, L.R., Arthur, M.A., Zachos, J.C., Paytan, A., 2003. Early Cenozoic decoupling of the global carbon and sulfur cycles. Paleoceanography, 18: 1090.

Langrock, U., Stein, R., 2004. Origin of marine petroleum source rocks from the Late Jurassic to Early Cretaceous Norwegian Greenland Seaway-evidence for stagnation and upwelling. Marine and Petroleum Geology, 21: 157-176.

Lassiter, J.C., Luhr, J.F., 2001. Osmium abundance and isotope variations in mafic Mexican volcanic rocks: Evidence for crustal contamination and constraints on the geochemical behavior of osmium during partial melting and fractional crystallization. Geochemistry, Geophysics, Geosystems, 2: 1027.

Levorsen, A.I., 2001. Reservoir Fluids - Water, Oil, Gas. Geology of Petroleum, 2. American Association of Petroleum Geologists Bulletin, Oklahoma, 724 pp.

Lewan, M.D., Maynard, J.B., 1982. Factors controlling enrichment of vanadium and nickel in the bitumen of organic sedimentary rocks. Geochimica et Cosmochimica Acta, 46: $2547-2560$. 
Li, Y., Zhang, S., Hobbs, R., Caiado, C., Sproson, A.D., Selby, D., Rooney, A.D., 2019. Monte Carlo sampling for error propagation in linear regression and applications in isochron geochronology. Science Bulletin, 64: 189-197.

Lillis, P.G., Selby, D., 2013. Evaluation of the rhenium-osmium geochronometer in the Phosphoria petroleum system, Bighorn Basin of Wyoming and Montana, USA. Geochimica et Cosmochimica Acta, 118: 312-330.

Lindner, M., Leich, D.A., Price Russ, G., Bazan, J.M., Borg, R.J., 1989. Direct determination of the half-life of ${ }^{187}$ Re. Geochimica et Cosmochimica Acta, 53: 1597-1606.

Littler, K., Röhl, U., Westerhold, T., Zachos, J.C., 2014. A high-resolution benthic stableisotope record for the South Atlantic: Implications for orbital-scale changes in Late Paleocene-Early Eocene climate and carbon cycling. Earth and Planetary Science Letters, 401: 18-30.

Liu, J., Pearson, D.G., 2014. Rapid, precise and accurate Os isotope ratio measurements of nanogram to sub-nanogram amounts using multiple Faraday collectors and amplifiers equipped with $1012 \Omega$ resistors by N-TIMS. Chemical Geology, 363: 301-311.

Liu, J., Selby, D., 2018. A matrix-matched reference material for validating petroleum Re-Os measurements. Geostandards and Geoanalytical Research, 42: 97-113.

Liu, J., Selby, D., Obermajer, M., Mort, A., 2018. Rhenium-osmium geochronology and oilsource correlation of the Duvernay petroleum system, Western Canada sedimentary basin: Implications for the application of the rhenium-osmium geochronometer to petroleum systems. American Association of Petroleum Geologists Bulletin, 102: 1627-1657.

Liu, J., Selby, D., Zhou, H., Pujol, M., 2019. Further evaluation of the Re-Os systematics of crude oil: Implications for Re-Os geochronology of petroleum systems. Chemical Geology, 513: 1-22.

Liu, Y., Masdua, A., Inoue, M., 1997. Substantial relief from oxygen isotope-related isobaric interference in negative thermal ionization mass spectrometry. Analytical Communications, 34: 171-173.

Liu, Z., Horton, D.E., Tabor, C., Sageman, B.B., Percival, L.M.E., Gill, B.C., Selby, D., 2019a. Assessing the contributions of comet impact and volcanism toward the climate perturbations of the Paleocene-Eocene Thermal Maximum. Geophysical Research Letters, 46: 14798-14806.

Liu, Z., Selby, D., Hackley, P.C., Over, D.J., 2020. Evidence of wildfires and elevated atmospheric oxygen at the Frasnian-Famennian boundary in New York (USA): Implications for the Late Devonian mass extinction. Geological Society of America Bulletin, In press.

Liu, Z., Selby, D., Zhang, H., Zheng, Q., Shen, S., Sageman, B.B., Grasby, S.E., Beauchamp, B., 2019b. Osmium-isotope evidence for volcanism across the WuchiapingianChanghsingian boundary interval. Chemical Geology, 529: 119313.

Luck, J.M., Allègre, C.J., 1982. The study of molybdenites through the ${ }^{187}{ }^{R e}-{ }^{187}$ Os chronometer. Earth and Planetary Science Letters, 61: 291-296.

Ludwig, K.R., 1980. Calculation of uncertainties of U-Pb isotope data. Earth and Planetary Science Letters, 46: 212-220.

Ludwig, K.R., 2012. Isoplot, version 3.75: A geochronological Toolkit for Microsoft Excel. Berkeley Geochronology Center Special Publication No. 5.

Luguet, A., Nowell, G.M., Pearson, D.G., 2008. ${ }^{184} \mathrm{Os} /{ }^{188} \mathrm{Os}$ and ${ }^{186} \mathrm{Os} /{ }^{188} \mathrm{Os}$ measurements by Negative Thermal Ionisation Mass Spectrometry (N-TIMS): Effects of interfering element and mass fractionation corrections on data accuracy and precision. Chemical Geology, 248: 342-362. 
Lus, W.Y., McDougall, I., Davies, H.L., 2004. Age of the metamorphic sole of the Papuan Ultramafic Belt ophiolite, Papua New Guinea. Tectonophysics, 392: 85-101.

Magoon, L.B., Dow, W.G., 1994. The Petroleum System-From Source to Trap, 60. American Association of Petroleum Geologists Bulletin, Oklahoma, 644 pp.

Mahdaoui, F., Michels, R., Reisberg, L., Pujol, M., Poirier, Y., 2015. Behavior of Re and Os during contact between an aqueous solution and oil: Consequences for the application of the Re-Os geochronometer to petroleum. Geochimica et Cosmochimica Acta, 158: $1-21$.

Mahdaoui, F., Reisberg, L., Michels, R., Hautevelle, Y., Poirier, Y., Girard, J.P., 2013. Effect of the progressive precipitation of petroleum asphaltenes on the Re-Os radioisotope system. Chemical Geology, 358: 90-100.

Manzano-Kareah, K., Sykes, R., 2008. Geochemical database and interpretations of Taranaki oils and condensates - Non-exclusive final report. GNS Science consultancy report 2008/16, 76 pp.

Martin, C.E., Peucker-Ehrenbrink, B., Brunskill, G., Szymczak, R., 2001. Osmium isotope geochemistry of a tropical estuary. Geochimica et Cosmochimica Acta, 65: 3193-3200.

Martini, E., 1971. Standard Tertiary and Quaternary calcareous nannoplankton zonation. In: Farinacci, A.E. (Editor), Proceedings of the Second Planktonic Conference Roma, Edizioni Tecnoscienza, Rome, pp. 739-785.

Matthews, K.J., Maloney, K.T., Zahirovic, S., Williams, S.E., Seton, M., Müller, R.D., 2016. Global plate boundary evolution and kinematics since the late Paleozoic. Global and Planetary Change, 146: 226-250.

Matusiewicz, H., 2003. Wet Digestion Methods. In: Mester, Z., Sturgeon, R. (Eds.), Sample Preparation for Trace Element Analysis. Elsevier, pp. 193-233.

McArthur, J.M., Algeo, T.J., van de Schootbrugge, B., Li, Q., Howarth, R.J., 2008. Basinal restriction, black shales, Re-Os dating, and the Early Toarcian (Jurassic) oceanic anoxic event. Paleoceanography, 23: doi.org/10.1029/2008PA001607.

Meisel, T., Moser, J., Fellner, N., Wegscheider, W., Schoenberg, R., 2001. Simplified method for the determination of $\mathrm{Ru}, \mathrm{Pd}, \mathrm{Re}, \mathrm{Os}$, Ir and $\mathrm{Pt}$ in chromitites and other geological materials by isotope dilution ICP-MS and acid digestion. Analyst, 126: 322-328.

Miller, C.A., Peucker-Ehrenbrink, B., Ball, L., 2009. Precise determination of rhenium isotope composition by multi-collector inductively-coupled plasma mass spectrometry. Journal of Analytical Atomic Spectrometry, 24: 1069-1078.

Minami, Y., Imura, T., Hayashi, S., Ohba, T., 2016. Mineralogical study on volcanic ash of the eruption on September 27, 2014 at Ontake volcano, central Japan: correlation with porphyry copper systems. Earth, Planets and Space, 68: doi.org/10.1186/s40623-0160440-2.

Moore, L.J., Machlan, L.A., Shields, W.R., Garner, E.L., 1974. Internal normalization techniques for high accuracy isotope dilution analyses. Application to molybdenum and nickel in standard reference materials. Analytical Chemistry, 46: 1082-1089.

Moore, P.R., 1988. Stratigraphy, composition, and environment of deposition of the Whangai Formation and associated Late Cretaceous-Paleocene rocks, eastern North Island, New Zealand. New Zealand Geological Survey Bulletin, 100, 82 pp.

Moore, P.R., 1989. Stratigraphy of the Waipawa Black Shale (Paleocene), eastern North Island, New Zealand. New Zealand Geological Survey record, 38, 19 pp.

Morford, J.L., Emerson, S., 1999. The geochemistry of redox sensitive trace metals in sediments. Geochimica et Cosmochimica Acta, 63: 1735-1750.

Morgan, J.W., 1986. Ultramafic xenoliths: Clues to Earth's late accretionary history. Journal of Geophysical Research: Solid Earth, 91: 12375-12387. 
Morgans, H.E.G., Pocknall, D.T., 1991. Biostratigraphy of Kupe South-5 offshore petroleum well, South Taranaki Bight. DSIR Geology and Geophysics contract report 1991/9, 41 pp.

Müller, P.J., Suess, E., 1979. Productivity, sedimentation rate, and sedimentary organic matter in the oceans-I. Organic carbon preservation. Deep Sea Research Part A. Oceanographic Research Papers, 26: 1347-1362.

Murray, D., McGregor, A., 1998. Ohanga-2 well completion report, PPL 38705. Ministry of Economic Development, New Zealand, unpublished Petroleum Report 2396, 1594 pp.

Naeher, S., Hollis, C.J., Clowes, C.D., Ventura, G.T., Shepherd, C.L., Crouch, E.M., Morgans, H.E.G., Bland, K.J., Strogen, D.P., Sykes, R., 2019. Depositional and organofacies influences on the petroleum potential of an unusual marine source rock: Waipawa Formation (Paleocene) in southern East Coast Basin, New Zealand. Marine and Petroleum Geology, 104: 468-488.

Naldrett, S.N., Libby, W.F., 1948. Natural radioactivity of rhenium. Physical Review, 73: 487493.

Nanne, J.A.M., Millet, M.-A., Burton, K.W., Dale, C.W., Nowell, G.M., Williams, H.M., 2017. High precision osmium stable isotope measurements by double spike MC-ICP-MS and N-TIMS. Journal of Analytical Atomic Spectrometry, 32: 749-765.

New Zealand Petroleum and Minerals, 2014. New Zealand Petroleum Basins. Ministry of Business, Innovation and Employment, Wellington, New Zealand, 108 pp.

Nowell, G.M., Luguet, A., Pearson, D.G., Horstwood, M.S.A., 2008. Precise and accurate ${ }^{186} \mathrm{Os} /{ }^{188} \mathrm{Os}$ and ${ }^{187} \mathrm{Os} /{ }^{188} \mathrm{Os}$ measurements by multi-collector plasma ionisation mass spectrometry (MC-ICP-MS) part I: Solution analyses. Chemical Geology, 248: 363393.

Nozaki, T., Suzuki, K., Ravizza, G., Kimura, J.-I., Chang, Q., 2012. A method for rapid determination of Re and Os isotope compositions using ID-MC-ICP-MS combined with the sparging method. Geostandards and Geoanalytical Research, 36: 131-148.

Ouellette, R.J., Rawn, J.D., 2015. Alkanes and Cycloalkanes. In: Ouellette, R.J., Rawn, J.D. (Eds.), Principles of Organic Chemistry. Elsevier, Boston, pp. 65-94.

Ownsworth, E., Selby, D., Ottley, C.J., Unsworth, E., Raab, A., Feldmann, J., Sproson, A.D., Kuroda, J., Faidutti, C., Bücker, P., 2019. Tracing the natural and anthropogenic influence on the trace elemental chemistry of estuarine macroalgae and the implications for human consumption. Science of The Total Environment, 685: 259-272.

Paquay, F.S., Ravizza, G., 2012. Heterogeneous seawater ${ }^{187} \mathrm{Os} /{ }^{188} \mathrm{Os}$ during the Late Pleistocene glaciations. Earth and Planetary Science Letters, 349-350: 126-138.

Paquay, F.S., Ravizza, G.E., Dalai, T.K., Peucker-Ehrenbrink, B., 2008. Determining chondritic impactor size from the marine osmium isotope record. Science, 320: 214 218.

Patterson, K.Y., Veillon, C., O'Haver, T.C., 1994. Error propagation in isotope dilution analysis as determined by Monte Carlo simulation. Analytical Chemistry, 66: 2829-2834.

Paul, M., Reisberg, L., Vigier, N., Zheng, Y., Ahmed, K.M., Charlet, L., Huq, M.R., 2010. Dissolved osmium in Bengal plain groundwater: Implications for the marine Os budget. Geochimica et Cosmochimica Acta, 74: 3432-3448.

Pegram, W.J., Krishnaswami, S., Ravizza, G.E., Turekian, K.K., 1992. The record of sea water ${ }^{187} \mathrm{Os} /{ }^{186}$ Os variation through the Cenozoic. Earth and Planetary Science Letters, 113: 569-576.

Pegram, W.J., Turekian, K.K., 1999. The osmium isotopic composition change of Cenozoic sea water as inferred from a deep-sea core corrected for meteoritic contributions. Geochimica et Cosmochimica Acta, 63: 4053-4058. 
Percival, L.M.E., Cohen, A.S., Davies, M.K., Dickson, A.J., Hesselbo, S.P., Jenkyns, H.C., Leng, M.J., Mather, T.A., Storm, M.S., Xu, W., 2016. Osmium isotope evidence for two pulses of increased continental weathering linked to Early Jurassic volcanism and climate change. Geology, 44: 759-762.

Peters, K., 1986. Guidelines for evaluating petroleum source rock using programmed pyrolysis. American Association of Petroleum Geologists Bulletin, 70: 318-329.

Peters, K.E., Walters, C.C., Moldowan, J.M., 2005. The Biomarker Guide: Volume 2: Biomarkers and Isotopes in Petroleum Systems and Earth History, 2. Cambridge University Press, Cambridge, 700 pp.

Peucker-Ehrenbrink, B., Hannigan, R.E., 2000. Effects of black shale weathering on the mobility of rhenium and platinum group elements. Geology, 28: 475-478.

Peucker-Ehrenbrink, B., Jahn, B., 2001. Rhenium-osmium isotope systematics and platinum group element concentrations: Loess and the upper continental crust. Geochemistry Geophysics Geosystems, 2: doi.org/10.1029/2001GC000172.

Peucker-Ehrenbrink, B., Ravizza, G., 2000. The marine osmium isotope record. Terra Nova, 12: $205-219$.

Peucker-Ehrenbrink, B., Ravizza, G., 2012. Chapter 8 - Osmium Isotope Stratigraphy. In: Gradstein, F.M., Ogg, J.G., Schmitz, M.D., Ogg, G.M. (Eds.), The Geologic Time Scale. Elsevier, Boston, pp. 145-166.

Pierpont, R., Wunderlich, A., Mayer, J., 2017. PEP 60089 Petroleum system and migration interpretation report. Ministry of Business, Innovation and Employment, New Zealand, unpublished Petroleum Report 5514, 22 pp.

Pierson-Wickmann, A.-C., Reisberg, L., France-Lanord, C., 2002. Behavior of Re and Os during low-temperature alteration: Results from Himalayan soils and altered black shales. Geochimica et Cosmochimica Acta, 66: 1539-1548.

Platzner, I., 1999. Modern Isotope Ratio Mass Spectrometry, $2^{\text {nd }}$ Ed. Wiley, Chichester, UK, $530 \mathrm{pp}$.

Poirier, A., 2006. Re-Os and $\mathrm{Pb}$ isotope systematics in reduced fjord sediments from Saanich Inlet (Western Canada). Earth and Planetary Science Letters, 249: 119-131.

Poirier, A., Doucelance, R., 2009. Effective correction of mass bias for rhenium measurements by MC-ICP-MS. Geostandards and Geoanalytical Research, 33: 195-204.

Poirier, A., Hillaire-Marcel, C., 2011. Improved Os-isotope stratigraphy of the Arctic Ocean. Geophysical Research Letters, 38: L14607.

Poplavko, Y.M., Ivanov, V.V., Karasik, T.G., Miller, A.D., Fadeyeva, V.A., Orekhov, V.S., Taliyev, S.D., Tarkhov, Y.A., 1975. On the concentration of rhenium in petroleum, petroleum bitumens and oil shales. Geochemistry International, 11: 969-972.

Porter, S.J., Selby, D., Suzuki, K., Gröcke, D., 2013. Opening of a trans-Pangaean marine corridor during the Early Jurassic: Insights from osmium isotopes across the Sinemurian-Pliensbachian GSSP, Robin Hood's Bay, UK. Palaeogeography, Palaeoclimatology, Palaeoecology, 375: 50-58.

Racionero-Gómez, B., Sproson, A.D., Selby, D., Gannoun, A., Gröcke, D.R., Greenwell, H.C., Burton, K.W., 2017. Osmium uptake, distribution, and ${ }^{187} \mathrm{Os} /{ }^{188} \mathrm{Os}$ and ${ }^{187} \mathrm{Re} /{ }^{188} \mathrm{Os}$ compositions in Phaeophyceae macroalgae, Fucus vesiculosus: Implications for determining the ${ }^{187} \mathrm{Os} /{ }^{188} \mathrm{Os}$ composition of seawater. Geochimica et Cosmochimica Acta, 199: 48-57.

Racionero-Gómez, B., Sproson, A.D., Selby, D., Gröcke, D.R., Redden, H., Greenwell, H.C., 2016. Rhenium uptake and distribution in phaeophyceae macroalgae, Fucus vesiculosus. Royal Society Open Science, 3: 160161. 
Rad, F., 2015. Romney-1 well completion report. Ministry of Economic Development New Zealand, unpublished Petroleum Report 4951, 980 pp.

Raine, I., 2004. Palynology of coal seam samples, Pakawau Group. GNS Science unpublished internal report JIR 2004/5, 19 pp.

Rattenbury, M.S., Cooper, R.A., Johnston, M.R., Forsyth, P.J., 1988. Geology of the Nelson area. Institute of Geological \& Nuclear Sciences 1:250 000 geological map 9. Institute of Geological \& Nuclear Sciences Limited, Lower Hutt, New Zealand, 67 pp.

Ravizza, G., 1993. Variations of the ${ }^{187} \mathrm{Os} /{ }^{186}$ Os ratio of seawater over the past 28 million years as inferred from metalliferous carbonates. Earth and Planetary Science Letters, 118: 335-348.

Ravizza, G., 2007. Reconstructing the marine ${ }^{187} \mathrm{Os} /{ }^{188}$ Os record and the particulate flux of meteoritic osmium during the late Cretaceous. Geochimica et Cosmochimica Acta, 71: 1355-1369.

Ravizza, G., Esser, B.K., 1993. A possible link between the seawater osmium isotope record and weathering of ancient sedimentary organic matter. Chemical Geology, 107: 255258.

Ravizza, G., Norris, R.N., Blusztajn, J., Aubry, M.P., 2001. An osmium isotope excursion associated with the Late Paleocene Thermal Maximum: Evidence of intensified chemical weathering. Paleoceanography and Paleoclimatology 16: 133-234.

Ravizza, G., Peucker-Ehrenbrink, B., 2003a. Chemostratigraphic evidence of Deccan volcanism from the marine osmium isotope record. Science 302: 1392-1395.

Ravizza, G., Peucker-Ehrenbrink, B., 2003b. The marine ${ }^{187} \mathrm{Os} /{ }^{188} \mathrm{Os}$ record of the EoceneOligocene transition: the interplay of weathering and glaciation. Earth and Planetary Science Letters, 210: 151-165.

Ravizza, G., Pyle, D., 1997. PGE and Os isotopic analyses of single sample aliquots with NiS fire assay preconcentration. Chemical Geology, 141: 251-268.

Ravizza, G., Turekian, K.K., 1989. Application of the ${ }^{187}$ Re- ${ }^{187}$ Os system to black shale geochronometry. Geochimica et Cosmochimica Acta, 53: 3257-3262.

Ravizza, G., Turekian, K.K., Hay, B.J., 1991. The geochemistry of rhenium and osmium in recent sediments from the Black Sea. Geochimica et Cosmochimica Acta, 55: 37413752.

Ravizza, G., VonderHaar, D., 2012. A geochemical clock in earliest Paleogene pelagic carbonates based on the impact-induced Os isotope excursion at the CretaceousPaleogene boundary. Paleoceanography, 27: PA3219.

Reed, J.D., 1992. Exploration geochemistry of the Taranaki Basin with emphasis on Kora, 1992 New Zealand Oil Exploration Conference Proceedings. Ministry of Commerce, 364-372 pp.

Reilly, C., Nicol, A., Walsh, J.J., Kroeger, K.F., 2016. Temporal changes of fault seal and early charge of the Maui Gas-condensate field, Taranaki Basin, New Zealand. Marine and Petroleum Geology, 70: 237-250.

Reilly, C., Nicol, A., Walsh, J.J., Seebeck, H., 2015. Evolution of faulting and plate boundary deformation in the Southern Taranaki Basin, New Zealand. Tectonophysics, 651-652: $1-18$.

Reisberg, L., Meisel, T., 2002. The Re-Os isotopic system: A review of analytical techniques. Geostandards Newsletter, 26: 249-267.

Rice, C.M., Mark, D.F., Selby, D., Neilson, J.E., Davidheiser-Kroll, B., 2016. Age and geologic setting of quartz vein-hosted gold mineralization at Curraghinalt, Northern Ireland: Implications for genesis and classification. Economic Geology, 111: 127-150. 
Righter, K., Chesley, J.T., Geist, D., Ruiz, J., 1998. Behavior of Re during magma fractionation: an example from Volcán Alcedo, Galápagos. Journal of Petrology, 39: 785-795.

Robinson, N., Ravizza, G., Coccioni, R., Peucker-Ehrenbrink, B., Norris, R.N., 2009. A highresolution marine ${ }^{187} \mathrm{Os} /{ }^{188} \mathrm{Os}$ record for the late Maastrichtian: Distinguishing the chemical fingerprints of Deccan volcanism and the KP impact event. Earth and Planetary Science Letters, 281: 159-168.

Rogers, K.M., Collen, J.D., Johnston, J.H., Elgar, N.E., 1999. A geochemical appraisal of oil seeps from the East Coast Basin, New Zealand. Organic Geochemistry, 30: 593-605.

Rolewicz, Z.L., 2013. Seawater osmium isotope records from Pacific ODP and IODP Sites refining the Paleogene curve and dating Red Clay sequences. Undergraduate research scholars Thesis, Texas A\&M University.

Rooney, A.D., Austermann, J., Smith, E.F., Li, Y., Selby, D., Dehler, C.M., Schmitz, M.D., Karlstrom, K.E., Macdonald, F.A., 2017. Coupled Re-Os and U-Pb geochronology of the Tonian Chuar Group, Grand Canyon. Geological Society of America Bulletin, 130: 1085-1098.

Rooney, A.D., Chew, D.M., Selby, D., 2011. Re-Os geochronology of the NeoproterozoicCambrian Dalradian Supergroup of Scotland and Ireland: Implications for Neoproterozoic stratigraphy, glaciations and Re-Os systematics. Precambrian Research, 185: 202-214.

Rooney, A.D., Macdonald, F.A., Strauss, J.V., Dudás, F.Ö., Hallmann, C., Selby, D., 2014. Re-Os geochronology and coupled Os-Sr isotope constraints on the Sturtian snowball Earth. Proceedings of the National Academy of Sciences, 111: 51-54.

Rooney, A.D., Selby, D., Houzay, J.P., Renne, P.R., 2010. Re-Os geochronology of a Mesoproterozoic sedimentary succession, Taoudeni Basin, Mauritania: Implications for basin-wide correlations and Re-Os organic-rich sediments systematics. Earth and Planetary Science Letters, 289: 486-496.

Rooney, A.D., Selby, D., Lewan, M.D., Lillis, P.G., Houzay, J.P., 2012. Evaluating Re-Os systematics in organic-rich sedimentary rocks in response to petroleum generation using hydrous pyrolysis experiments. Geochimica et Cosmochimica Acta, 77: 275-291.

Rooney, A.D., Selby, D., Lloyd, J.M., Roberts, D.H., Lückge, A., Sageman, B.B., Prouty, N.G., 2016. Tracking millennial-scale Holocene glacial advance and retreat using osmium isotopes: Insights from the Greenland ice sheet. Quaternary Science Reviews, 138: 4961.

Roy-Barman, M., Wasserburg, G.J., Papanastassiou, D.A., Chaussidon, M., 1998. Osmium isotopic compositions and Re-Os concentrations in sulfide globules from basaltic glasses. Earth and Planetary Science Letters, 154: 331-347.

Rudnick, R.L., Gao, S., 2003. Composition of the Continental Crust A2 - Holland, Heinrich D. In: Turekian, K.K. (Ed.), Treatise on Geochemistry. Pergamon, Oxford, pp. 1-64.

Russ, G.P., Bazan, J.M., Date, A.R., 1987. Osmium isotopic ratio measurements by inductively coupled plasma source mass spectrometry. Analytical Chemistry, 59: 984-989.

Russell, W.A., Papanastassiou, D.A., Tombrello, T.A., 1978. Ca isotope fractionation on the Earth and other solar system materials. Geochimica et Cosmochimica Acta, 42: 10751090.

Scarlett, A.G., Holman, A.I., Georgiev, S.V., Stein, H.J., Summons, R.E., Grice, K., 2019. Multi-spectroscopic and elemental characterization of southern Australian asphaltites. Organic Geochemistry, 133: 77-91.

Schiøler, P., Rogers, K.M., Sykes, R., Hollis, C.J., Ilg, B., Meadows, D., Roncaglia, L., Uruski, C.I., 2010. Palynofacies, organic geochemistry and depositional environment of the 
Tartan Formation (Late Paleocene), a potential source rock in the Great South Basin, New Zealand. Marine and Petroleum Geology, 27: 351-369.

Schlumberger, 2017. 1D Petroleum system modelling onshore East Coast Basin, New Zealand. Ministry of Business, Innovation and Employment, New Zealand, unpublished Petroleum Report 5404, 43 pp.

Schmitz, B., Peucker-Ehrenbrink, B., Heilmann-Clausen, C., Åberg, G., Asaro, F., Lee, C.T.A., 2004. Basaltic explosive volcanism, but no comet impact, at the PaleoceneEocene boundary: high-resolution chemical and isotopic records from Egypt, Spain and Denmark. Earth and Planetary Science Letters, 225: 1-17.

Schoenberg, R., Nägler, T.F., Kramers, J.D., 2000. Precise Os isotope ratio and Re-Os isotope dilution measurements down to the picogram level using multicollector inductively coupled plasma mass spectrometry. International Journal of Mass Spectrometry, 197: 85-94.

Schröder-Adams, C.J., Herrle, J.O., Selby, D., Quesnel, A., Froude, G., 2019. Influence of the High Arctic Igneous Province on the Cenomanian/Turonian boundary interval, Sverdrup Basin, High Canadian Arctic. Earth and Planetary Science Letters, 511: 7688.

Selby, D., 2007. Direct rhenium-osmium age of the Oxfordian-Kimmeridgian boundary, Staffin Bay, lsle of Skye, U.K., and the late Jurassic time scale. Norsk Geologisk Tidsskrift, 87: 291-299.

Selby, D., Creaser, R., Dewing, K., Fowler, M., 2005a. Evaluation of bitumen as a Re-Os geochronometer for hydrocarbon maturation and migration: A test case from the Polaris MVT deposit, Canada. Earth and Planetary Science Letters, 235: 1-15.

Selby, D., Creaser, R.A., 2003. Re-Os geochronology of organic rich sediments: an evaluation of organic matter analysis methods. Chemical Geology, 200: 225-240.

Selby, D., Creaser, R.A., 2005a. Direct radiometric dating of hydrocarbon deposits using rhenium-osmium isotopes. Science, 308: 1293-1295.

Selby, D., Creaser, R.A., 2005b. Direct radiometric dating of the Devonian-Mississippian timescale boundary using the Re-Os black shale geochronometer. Geology, 33: 545-548.

Selby, D., Creaser, R.A., Dewing, K., Fowler, M., 2005b. Evaluation of bitumen as a ${ }^{187} \mathrm{Re}-$ ${ }^{187}$ Os geochronometer for hydrocarbon maturation and migration: A test case from the Polaris MVT deposit, Canada. Earth and Planetary Science Letters, 235: 1-15.

Selby, D., Creaser, R.A., Fowler, M.G., 2007a. Re-Os elemental and isotopic systematics in crude oils. Geochimica et Cosmochimica Acta, 71: 378-386.

Selby, D., Creaser, R.A., Stein, H.J., Markey, R.J., Hannah, J.L., 2007b. Assessment of the ${ }^{187} \mathrm{Re}$ decay constant by cross calibration of Re-Os molybdenite and U-Pb zircon chronometers in magmatic ore systems. Geochimica et Cosmochimica Acta, 71: 19992013.

Selby, D., Mutterlose, J., Condon, D.J., 2009. U-Pb and Re-Os geochronology of the Aptian/Albian and Cenomanian/Turonian stage boundaries: Implications for timescale calibration, osmium isotope seawater composition and Re-Os systematics in organicrich sediments. Chemical Geology, 265: 394-409.

Shen, J.J., Papanastassiou, D.A., Wasserburg, G.J., 1996. Precise Re-Os determinations and systematics of iron meteorites. Geochimica et Cosmochimica Acta, 60: 2887-2900.

Sherwood, A.M., Lindqvist, J.K., Newman, J., Sykes, R., McCabe, P.J., Parrish, J.T., 1992. Depositional controls on Cretaceous coals and coal measures in New Zealand. In: McCabe, P.J., Parrish, J.T. (Eds.), Controls on the distribution and quality of Cretaceous coals. Geological Society of America Special Paper 267, 407 pp. 
Shirey, S.B., Walker, R.J., 1995. Carius tube digestion for low-blank rhenium-osmium analysis. Analytical Chemistry, 67: 2136-2141.

Shirey, S.B., Walker, R.J., 1998. The Re-Os isotope system in cosmochemistry and hightemperature geochemistry. Annual Review of Earth and Planetary Sciences, 26: 423500.

Simmonds, V., Moazzen, M., Selby, D., 2019. U-Pb zircon and Re-Os molybdenite age of the Siah Kamar porphyry molybdenum deposit, NW Iran. International Geology Review, 61: 1786-1802.

Smoliar, M.I., Walker, R.J., Morgan, J.W., 1996. Re-Os ages of group IIA, IIIA, IVA, and IVB iron meteorites. Science, 271: 1099-1102.

Speight, J.G., 2004. Petroleum asphaltenes - part 1: Asphaltenes, resins and the structure of petroleum. Oil \& Gas Science and Technology, 59: 467-477.

Sperling, E.A., Rooney, A.D., Hays, L., Sergeev, V.N., Vorob'eva, N.G., Sergeeva, N.D., Selby, D., Johnston, D.T., Knoll, A.H., 2014. Redox heterogeneity of subsurface waters in the Mesoproterozoic ocean. Geobiology, 12: 373-386.

Sproson, A.D., Selby, D., Gannoun, A., Burton, K.W., Dellinger, M., Lloyd, J.M., 2018. Tracing the impact of coastal water geochemistry on the Re-Os systematics of macroalgae: Insights from the Basaltic Terrain of Iceland. Journal of Geophysical Research: Biogeosciences, 123: 2791-2806.

Sproson, A.D., Selby, D., Suzuki, K., Oda, T., Kuroda, J., 2020. Anthropogenic Osmium in Macroalgae from Tokyo Bay Reveals Widespread Contamination from Municipal Solid Waste. Environmental Science \& Technology, 54: 9356-9365.

Stagpoole, V., Nicol, A., 2008. Regional structure and kinematic history of a large subduction back thrust: Taranaki Fault, New Zealand. Journal of Geophysical Research: Solid Earth, 113: B01403.

Standards Australia, 2002. AS 1038.11-2002, coal and coke - analysis and testing coal - forms of sulfur, Standards Australia, Sydney, New South Wales, 16 pp.

Stein, H., Hannah, J., 2014. Rhenium-Osmium Geochronology: Sulfides, Shales, Oils, and Mantle. In: Rink, J., Thompson, J. (Eds.), Earth Sciences Series, Encyclopedia of Scientific Dating Methods. Springer Netherlands, Dordrecht, pp. 1-25.

Stein, H.J., Markey, R.J., Morgan, J.W., Hannah, J.L., Scherstén, A., 2001. The remarkable Re-Os chronometer in molybdenite: how and why it works. Terra Nova, 13: 479-486.

Strogen, D.P., 2011. Updated paleogeographic maps for the Taranaki Basin and surrounds. GNS Science Report, 2010/53, 83 pp.

Strogen, D.P., Seebeck, H., Nicol, A., King, P.R., 2017. Two-phase Cretaceous-Paleocene rifting in the Taranaki Basin region, New Zealand; implications for Gondwana breakup. Journal of the Geological Society, 174: 929-946.

Suggate, R.P., 1959. New Zealand Coals: Their geological setting and its influence on their properties, Department of Scientific and Industrial Research Bulletin 134. Department of Scientific and Industrial Research, Wellington, New Zealand.

Sun, W., Bennett, V.C., Eggins, S.M., Kamenetsky, V.S., Arculus, R.J., 2003. Enhanced mantle-to-crust rhenium transfer in undegassed arc magmas. Nature, 422: 294-297.

Suzuki, K., Miyata, Y., Kanazawa, N., 2004. Precise Re isotope ratio measurements by negative thermal ionization mass spectrometry (NTI-MS) using total evaporation technique. International Journal of Mass Spectrometry, 235: 97-101.

Suzuki, K., Tatsumi, Y., 2006. Re-Os systematics of high-Mg andesites and basalts from the Setouchi volcanic belt, SW Japan: Implications for interaction between wedge mantle and slab-derived melt. Geochemical Journal, 40: 297-307. 
Sykes, R., 2001. Depositional and rank controls on the petroleum potential of coaly source rocks. In: Hill, K.C., Bernecker, T. (Eds.), Eastern Australasian Basins Symposium, A Refocused Energy Perspective for the Future. Petroleum Exploration Society of Australia, pp. 591-601.

Sykes, R., Bartram, K.M., Dow, M.J., Suggate, R.P., 1999. Enhanced petroleum potential of marine influenced coals in Mangahewa Formation, Taranaki Basin, Abstracts and Program, 16th Annual Meeting of the Society for Organic Petrology, 1999, Snowbird, Utah, USA, pp. 41-45.

Sykes, R., Dow, M.J., 2000. Petroleum source rock properties of North Cape Formation (Late Cretaceous) coaly sediments, Taranaki Basin, 2000 NZ Petroleum Conference Proceedings. Ministry of Economic Development, pp. 264-286.

Sykes, R., Raine, J.I., 2008. Organofacies controls on the oil potential of coaly source rocks in the Late Cretaceous North Cape Formation, Taranaki Basin. In: Blevin, J.E., Bradshaw, B.E., Uruski, C. (Eds.), Eastern Australasian basins symposium III. Petroleum Exploration Society of Australia, Special Publication, pp. 219-225.

Sykes, R., Snowdon, L.R., 2002. Guidelines for assessing the petroleum potential of coaly source rocks using Rock-Eval pyrolysis. Organic Geochemistry, 33: 1441-1455.

Sykes, R., Snowdon, L.R., Johansen, P.E., 2004. Leaf biomass - a new paradigm for sourcing the terrestrial oils of Taranaki Basin. In: Boult, P.J., Johns, D.R., Lang, S.C. (Eds.), Eastern Australasian Basins Symposium II. Petroleum Exploration Society of Australia, Special Publication, pp. 553-574.

Sykes, R., Volk, H., George, S.C., Ahmed, M., Higgs, K.E., Johansen, P.E., Snowdon, L.R., 2014. Marine influence helps preserve the oil potential of coaly source rocks: Eocene Mangahewa Formation, Taranaki Basin, New Zealand. Organic Geochemistry, 66: 140-163.

Sykes, R., Zink, K.-G., 2008. Geochemical study of four production oils from the Tui Fields and chemometric analysis of the wider Maui oil family, offshore Taranaki Basin. Ministry of Economic Development, New Zealand, unpublished Petroleum Report 3937, 134 pp.

Sykes, R., Zink, K.-G., 2012. The New Zealand oil geochemistry database. GNS Science Data Series $14 a$.

Sykes, R., Zink, K.-G., Rogers, K.M., Phillips, A., Ventura, G.T., 2012. New and updated geochemical databases for New Zealand petroleum samples, with assessments of genetic oil families, source age, facies and maturity. GNS Science consultancy report 2012/37, GNS Science Consultancy Report, 36 pp.

Tayler, M.J.S., 2011. Investigating stratigraphic evidence for Antarctic glaciation in the greenhouse world of the Paleocene, eastern North Island, New Zealand. Masters Thesis, University of Waikato.

Tejada, M.L.G., Kuroda, J., Suzuki, K., Ohkouchi, N., Sakamoto, T., Tatsumi, Y., Coccioni, R., Mahoney, J.J., 2009. Ontong Java Plateau eruption as a trigger for the early Aptian oceanic anoxic event. Geology, 37: 855-858.

Tessalina, S., Jourdan, F., Nunes, L., Kennedy, A., Denyszyn, S., Puchtel, I., Touboul, M., Creaser, R., Boyet, M., Belousova, E., Trinquier, A., 2015. Application of radiogenic isotopes in geosciences: Overview and perspectives. In: Grice, K. (Ed.), Principles and Practice of Analytical Techniques in Geosciences. The Royal Society of Chemistry, pp. 49-93.

Them, T.R., Gill, B.C., Selby, D., Gröcke, D.R., Friedman, R.M., Owens, J.D., 2017. Evidence for rapid weathering response to climatic warming during the Toarcian Oceanic Anoxic Event. Scientific Reports, 7: 5003. 
Thermo Fisher Scientific Inc., 2018. Thermo Scientific Triton XT TIMS, Waltham, Massachusetts, USA, 16 pp.

Thomas, D.J., Bralower, T.J., Jones, C.E., 2003. Neodymium isotopic reconstruction of late Paleocene-early Eocene thermohaline circulation. Earth and Planetary Science Letters, 209: 309-322.

Thrasher, G.P., 1992. Late Cretaceous geology of the Taranaki Basin, New Zealand, New Zealand. PhD thesis, Victoria University of Wellington.

Tissot, B.P., Welte, D.H., 1984. Petroleum Formation and Occurrence. Springer-Verlag, Berlin, 699 pp.

Titheridge, D.G., 1977. Stratigraphy and sedimentology of the Upper Pakawau and Lower Westhaven Groups (Upper Cretaceous- Oligocene), northwest Nelson. MSc thesis, University of Canterbury.

Torsvik, T.H., Van der Voo, R., Preeden, U., Mac Niocaill, C., Steinberger, B., Doubrovine, P.V., van Hinsbergen, D.J.J., Domeier, M., Gaina, C., Tohver, E., Meert, J.G., McCausland, P.J.A., Cocks, L.R.M., 2012. Phanerozoic polar wander, palaeogeography and dynamics. Earth-Science Reviews, 114: 325-368.

Tripathy, G.R., Hannah, J.L., Stein, H.J., 2018. Refining the Jurassic-Cretaceous boundary: Re-Os geochronology and depositional environment of Upper Jurassic shales from the Norwegian Sea. Palaeogeography, Palaeoclimatology, Palaeoecology, 503: 13-25.

Tripathy, G.R., Hannah, J.L., Stein, H.J., Geboy, N.J., Ruppert, L.F., 2015. Radiometric dating of marine-influenced coal using Re-Os geochronology. Earth and Planetary Science Letters, 432: 13-23.

Tripathy, G.R., Hannah, J.L., Stein, H.J., Yang, G., 2014. Re-Os age and depositional environment for black shales from the Cambrian-Ordovician boundary, Green Point, western Newfoundland. Geochemistry, Geophysics, Geosystems, 15: 1021-1037.

Tripathy, G.R., Singh, S.K., 2015. Re-Os depositional age for black shales from the Kaimur Group, Upper Vindhyan, India. Chemical Geology, 413: 63-72.

Turekian, K.K., Pegram, W.J., 1997. Os Isotope Record in a Cenozoic Deep-Sea Core: Its Relation to Global Tectonics and Climate. In: Ruddiman, W.F. (Ed.), Tectonic Uplift and Climate Change. Springer US, Boston, MA, pp. 383-397.

Turgeon, S.C., Creaser, R.A., 2008. Cretaceous oceanic anoxic event 2 triggered by a massive magmatic episode. Nature, 454: 323-326.

Turgeon, S.C., Creaser, R.A., Algeo, T.J., 2007. Re-Os depositional ages and seawater Os estimates for the Frasnian-Famennian boundary: Implications for weathering rates, land plant evolution, and extinction mechanisms. Earth and Planetary Science Letters, 261: 649-661.

Uruski, C.I., 2020. Seismic recognition of igneous rocks of the Deepwater Taranaki Basin, New Zealand, and their distribution. New Zealand Journal of Geology and Geophysics, 63: 190-209.

Uruski, C.1., 2008. Deepwater Taranaki, New Zealand: structural development and petroleum potential. Exploration Geophysics, 39: 94-107.

van Acken, D., Tütken, T., Daly, J.S., Schmid-Röhl, A., Orr, P.J., 2019. Rhenium-osmium geochronology of the Toarcian Posidonia Shale, SW Germany. Palaeogeography, Palaeoclimatology, Palaeoecology, 534: 109294.

Volk, H., George, S., Sykes, R., 2008. Influence of depositional environment and early diagenesis on molecular maturity parameters of coals - an example from the Taranaki Basin. In: Blevin, J.E., Bradshaw, B.E., Uruski, C. (Eds.), Eastern Australasian Basins Symposium III. Petroleum Exploration Society of Australia, Special Publication, pp. $215-218$. 
Völkening, J., Walczyk, T., G. Heumann, K., 1991. Osmium isotope ratio determinations by negative thermal ionization mass spectrometry. International Journal of Mass Spectrometry and Ion Processes, 105: 147-159.

Walker, R.J., 1988. Low-blank chemical separation of rhenium and osmium from gram quantities of silicate rock for measurement by resonance ionization mass spectrometry. Analytical Chemistry, 60: 1231-1234.

Walker, R.J., Carlson, R.W., Shirey, S.B., F.R, B., 1989. Os, Sr, Nd, and Pb isotope systematics of southern African peridotite xenoliths: Implications for the chemical evolution of subcontinental mantle. Geochimica et Cosmochimica Acta, 53: 1583-1595.

Walker, R.J., Fassett, J.D., 1986. Isotopic measurement of subnanogram quantities of rhenium and osmium by resonance ionization mass spectrometry. Analytical Chemistry (Washington), 58: 2923-2927.

Walker, R.J., Morgan, J.W., Beary, E.S., Smoliar, M.I., Czamanske, G.K., Horan, M.F., 1997. Applications of the ${ }^{190} \mathrm{Pt}-{ }^{186} \mathrm{Os}$ isotope system to geochemistry and cosmochemistry. Geochimica et Cosmochimica Acta, 61: 4799-4807.

Walters, C.C., Wang, F.C., Qian, K., Wu, C., Mennito, A.S., Wei, Z., 2015. Petroleum alteration by thermochemical sulfate reduction - A comprehensive molecular study of aromatic hydrocarbons and polar compounds. Geochimica et Cosmochimica Acta, 153: $37-71$.

Washburn, A.M., Hudson, S.M., Selby, D., Abdullayev, N., Shiyanova, N., 2019. Constraining the timing and depositional conditions of the Maikop Formation within the Kura Basin, Eastern Azerbaijan, through the application of Re-Os geochronology and chemostratigraphy Journal of Petroleum Geology, 42: 281-299.

Wasserburg, G.J., Papanastassiou, D.A., Nenow, E.V., Bauman, C.A., 1969. A programmable magnetic field mass spectrometer with online data processing. Review of Scientific Instruments, 40: 288-295.

Wendt, I., Carl, C., 1991. The statistical distribution of the mean squared weighted deviation. Chemical Geology: Isotope Geoscience section, 86: 275-285.

Westerhold, T., Röhl, U., Donner, B., McCarren, H.K., Zachos, J.C., 2011. A complete highresolution Paleocene benthic stable isotope record for the central Pacific (ODP Site 1209). Paleoceanography, 26: PA2216.

Westerhold, T., Röhl, U., Donner, B., Zachos, J.C., 2018. Global extent of early Eocene hyperthermal events: A new Pacific benthic foraminiferal isotope record from Shatsky Rise (ODP Site 1209). Paleoceanography and Paleoclimatology, 33: 626-642.

Westerhold, T., Röhl, U., Frederichs, T., Agnini, C., Raffi, I., Zachos, J.C., Wilkens, R.H., 2017. Astronomical calibration of the Ypresian timescale: implications for seafloor spreading rates and the chaotic behavior of the solar system? Climate of the Past, 13: 1129-1152.

Wieczorek, R., Fantle, M.S., Kump, L.R., Ravizza, G., 2013. Geochemical evidence for volcanic activity prior to and enhanced terrestrial weathering during the Paleocene Eocene Thermal Maximum. Geochimica et Cosmochimica Acta, 119: 391-410.

Wieser, M.E., Brand, W.A., 1999. Isotope Ratio Studies Using Mass Spectrometry. In: Lindon, J.C. (Ed.), Encyclopedia of Spectroscopy and Spectrometry. Elsevier, Oxford, pp. 1072-1086.

Williams, E.G., Keith, M.L., 1963. Relationship between sulfur in coals and the occurrence of marine roof beds. Economic Geology, 58: 720-729.

Wilson, G.J., Moore, P.R., 1988. Cretaceous-Tertiary boundary in the Te Hoe River area, western Hawkes Bay. New Zealand Geological Survey Record, 35: 34-37. 
Wizevich, M.C., 1994. Sedimentary evolution of the onshore Pakawau sub-basin: Rift sediments of the Taranaki Basin deposited during Tasman Sea spreading. In: Van der Lingen, G.J., Swanson, K.M., Muir, R.J. (Eds.), Evolution of the Tasman Sea Basin. A.A. Balkema, Rotterdam, pp. 83-104.

Wizevich, M.C., Thrasher, G.P., Bussell, M.R., Wilson, G.J., Collen, J.D., 1992. Evidence for marine deposition in the Late Cretaceous Pakawau group, northwest Nelson. New Zealand Journal of Geology and Geophysics, 35: 363-369.

Woodhouse, O.B., Ravizza, G., Kenison Falkner, K., Statham, P.J., Peucker-Ehrenbrink, B., 1999. Osmium in seawater: vertical profiles of concentration and isotopic composition in the eastern Pacific Ocean. Earth and Planetary Science Letters, 173: 223-233.

Woodland, S.J., Ottley, C.J., Pearson, D.G., Swarbrick, R.E., 2001. Microwave digestion of oils for analysis of platinum group and rare earth elements by ICP-MS. In: Holland, G., Tanner, S.D. (Eds.), Plasma Source Mass Spectrometry: The New Millennium. The Royal Society of Chemistry, pp. 17-24.

Xu, G., Hannah, J.L., Stein, H.J., Bingen, B., Yang, G., Zimmerman, A., Weitschat, W., Mørk, A., Weiss, H.M., 2009. Re-Os geochronology of Arctic black shales to evaluate the Anisian-Ladinian boundary and global faunal correlations. Earth and Planetary Science Letters, 288: 581-587.

Xu, W., Ruhl, M., Jenkyns, Hugh C., Hesselbo, Stephen P., Riding, James B., Selby, D., Naafs, B.David A., Weijers, Johan W.H., Pancost, Richard D., Tegelaar, Erik W., Idiz, Erdem F., 2017. Carbon sequestration in an expanded lake system during the Toarcian oceanic anoxic event. Nature Geoscience, 10: 129-134.

Yamashita, Y., Takahashi, Y., Haba, H., Enomoto, S., Shimizu, H., 2007. Comparison of reductive accumulation of Re and Os in seawater-sediment systems. Geochimica et Cosmochimica Acta, 71: 3458-3475.

Yang, G., Hannah, J.L., Zimmerman, A., Stein, H.J., Bekker, A., 2009. Re-Os depositional age for Archean carbonaceous slates from the southwestern Superior Province: Challenges and insights. Earth and Planetary Science Letters, 280: 83-92.

York, D., 1968. Least squares fitting of a straight line with correlated errors. Earth and Planetary Science Letters, 5: 320-324.

Zachos, J.C., Dickens, G.R., Zeebe, R.E., 2008. An early Cenozoic perspective on greenhouse warming and carbon-cycle dynamics. Nature, 451: 279-283.

Zaiss, J., Ravizza, G., Goderis, S., Sauvage, J., Claeys, P., Johnson, K., 2014. A complete Os excursion across a terrestrial Cretaceous-Paleogene boundary at the West Bijou Site, Colorado, with evidence for recent open system behavior. Chemical Geology, 385: 716.

Zheng, G., Suzuki, K., Kuno, A., Mastuo, M., Takano, B., Shimizu, H., 2014. Osmium geochemistry of modern estuarine sediments from the Tama and Yasaka rivers in Japan. Applied Geochemistry, 40: 82-88.

Zhu, B., Becker, H., Jiang, S.Y., Pi, D.H., Fischer-Gödde, M., Yang, J.H., 2013. Re-Os geochronology of black shales from the Neoproterozoic Doushantuo Formation, Yangtze platform, South China. Precambrian Research, 225: 67-76.

Zimbelman, D.R., Rye, R.O., Breit, G.N., 2005. Origin of secondary sulfate minerals on active andesitic stratovolcanoes. Chemical Geology, 215: 37-60. 


\section{Appendix A: Geographic distribution of New Zealand oil families}

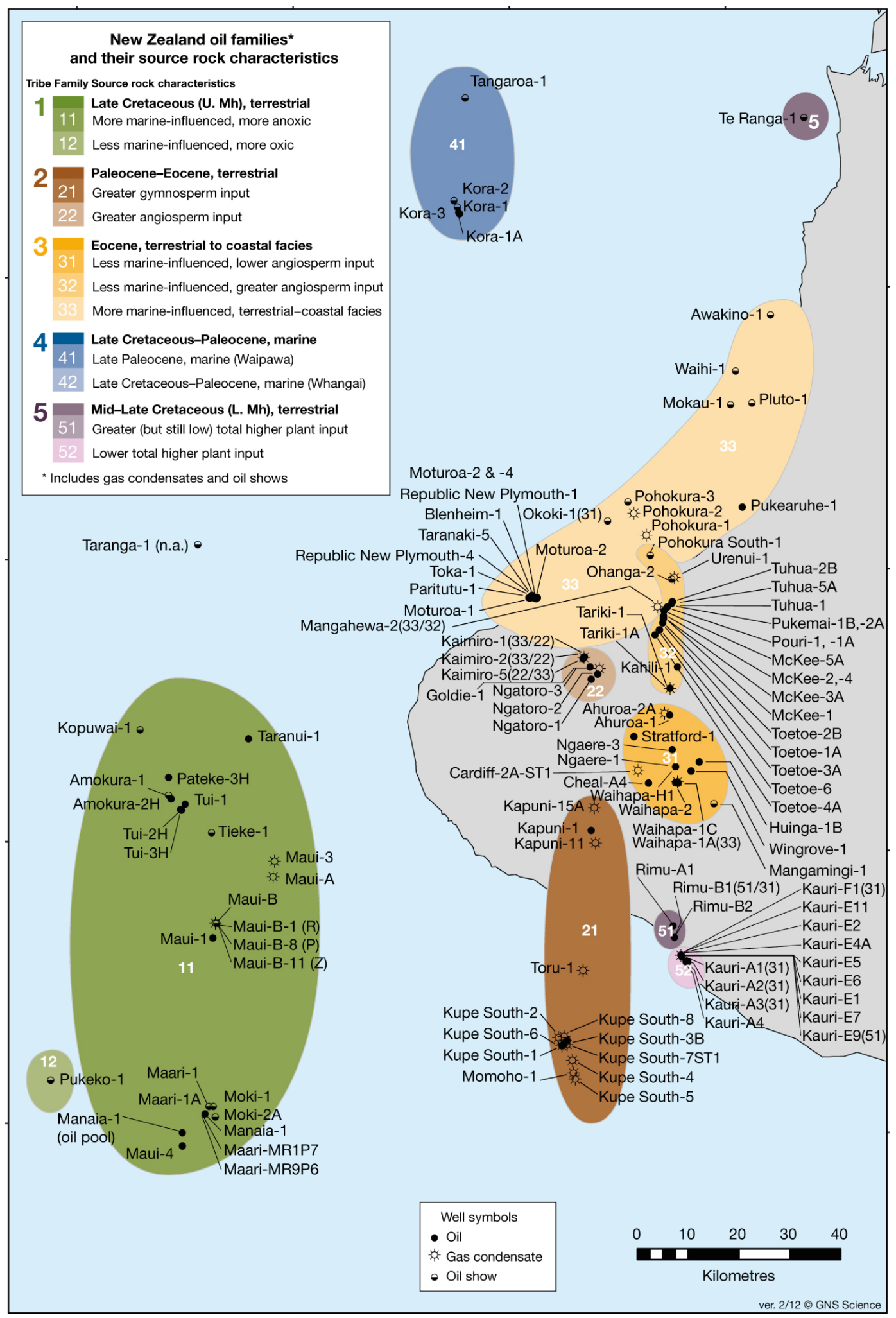

Fig. A.1. The geographic distribution of genetic oil families in the Taranaki Basin (Sykes et al., 2012). 


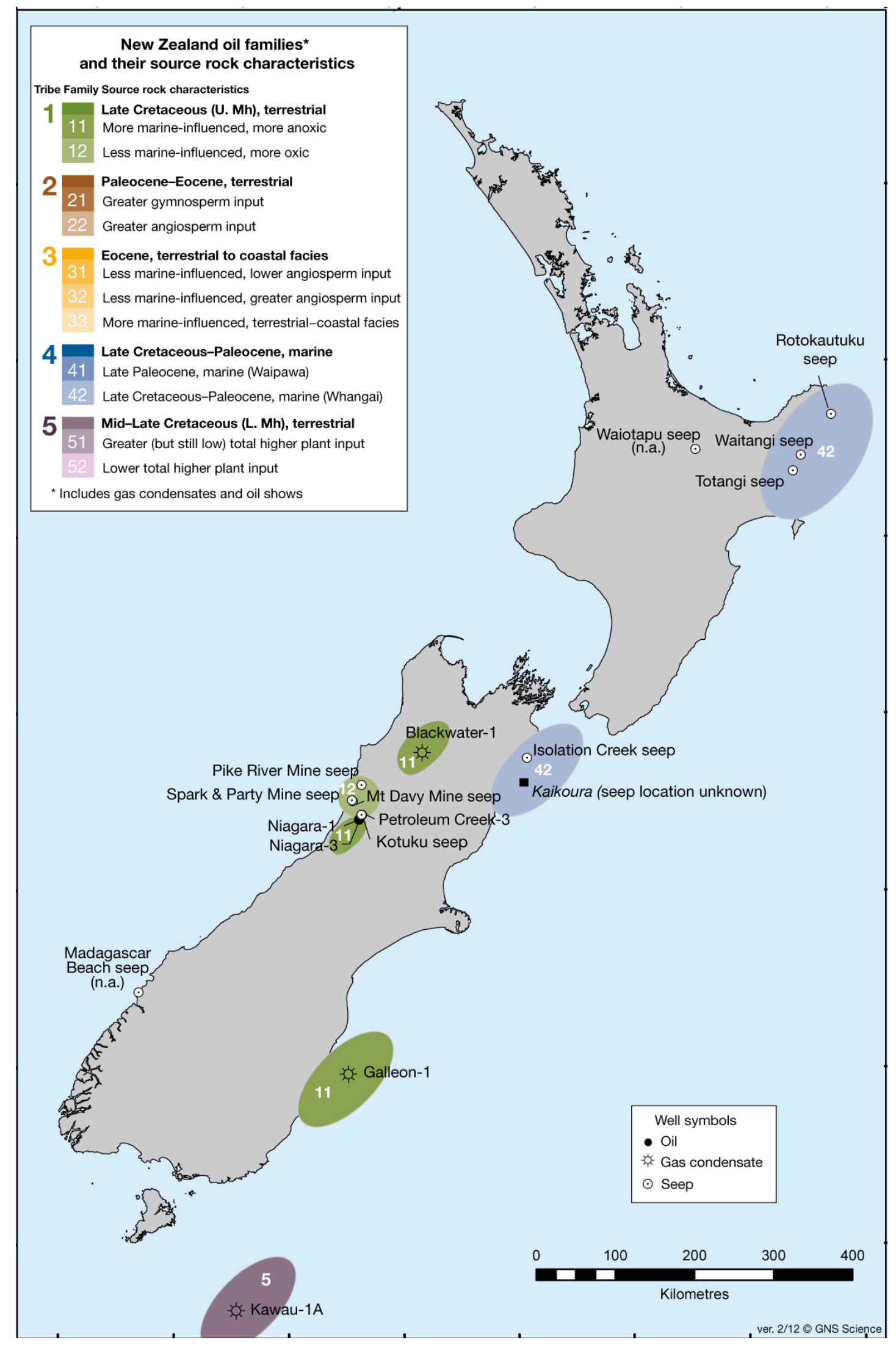

Fig. A.2. The geographic distribution of genetic oil families in New Zealand's other sedimentary basins (Sykes et al., 2012). 


\section{Appendix B: Rhenium test method and results}

\section{B.1 Re test method - VUW Geochemistry Laboratory}

The Re-test method was used as a sample screening process to determine the concentration of Re for sample spiking and to prioritise samples for full Re-Os geochemistry. All work was undertaken in Pico-Trace laminar flow workstations using ultra-pure reagents (e.g. Teflondistilled acids and $>18.2 \mathrm{M} \Omega$ water) unless otherwise noted.

\section{B.1.1 Acid dissolution of rock samples}

1. Approximately $0.1 \mathrm{~g}$ of rock powder was added onto a pre-cleaned Savillex ${ }^{\circledR}$ digestion beaker and spiked with a known amount $(0.05-0.1 \mathrm{~g})$ of ${ }^{185}$ Re enriched tracer solution.

2. A mix of $3 \mathrm{ml} \sim 12 \mathrm{~N} \mathrm{HCl}$ and $6 \mathrm{ml} 16 \mathrm{~N} \mathrm{HNO}_{3}$ (inverse aqua regia) was added to the beaker, which was then tightly closed and swirled to mix the sample and acid.

3. The sealed beaker was placed on a hot plate at $100^{\circ} \mathrm{C}$ overnight (at least $12 \mathrm{~h}$ ) to reflux.

4. After digestion, the sample was removed from the hot plate and left to cool in a hood.

5. Once cool, the beaker was inverted to take up any evaporation on the lid. For some samples, there was a large amount of undigested residue, which was removed by centrifuging and decanting back the solution into the beaker.

6. The beaker and lid were both placed on the hot plate to dry down overnight at $80^{\circ} \mathrm{C}$.

\section{B.1.2 Anion exchange chromatography}

\section{B.1.2.1 Column preparation}

1. Re was purified using anion exchange chemistry in disposable pipette columns.

2. The top and tip of the pipette were cut off and a frit fitted to the bottom.

3. The pipette was then filled with ultra-pure water and any air bubbles removed.

4. Eichrom 1X8, 100-200 mesh resin was then added until it filled approximately $4 \mathrm{~cm}$ of the pipette column. [The elution profile of Re for this length of resin in the column was earlier calibrated using a synthetic Re solution].

5. The resin was then washed with $4 \mathrm{ml}$ of $8 \mathrm{~N} \mathrm{HNO}_{3}$ and equilibrated with $4 \mathrm{ml}$ of $0.2 \mathrm{~N}$ $\mathrm{HNO}_{3}$. 


\section{B.1.2.2 Sample elution}

1. The dried sample was dissolved in $3 \mathrm{ml}$ of $0.2 \mathrm{~N} \mathrm{HNO}_{3}$ and then loaded onto the column using a clean pipette for each sample.

2. The stem walls were rinsed four times with $0.25 \mathrm{ml}$ of $0.2 \mathrm{~N} \mathrm{HNO}_{3}$ and twice with $1 \mathrm{ml}$ of $0.2 \mathrm{~N} \mathrm{HNO}_{3}$.

3. The sample-containing resin was then washed twice with $1 \mathrm{ml}$ of $0.2 \mathrm{~N} \mathrm{HCl}$ and twice again with $2 \mathrm{ml}$ of $6 \mathrm{~N} \mathrm{HNO}_{3}$.

4. At this point, the Re fraction was ready for collection in a pre-cleaned Teflon beaker using $4 \mathrm{ml}$ of $6 \mathrm{~N}^{-\mathrm{HNO}_{3}}$.

5. Lastly, the collected Re was dried on a hot plate at $\sim 80^{\circ} \mathrm{C}$.

\section{B.1.3 Isotope dilution-ICP-MS}

The dried Re-cut was dissolved in $3 \mathrm{ml}$ of $0.2 \mathrm{~N} \mathrm{HNO}_{3}$ and Re concentration determined by isotope dilution on a Thermo Fisher Scientific Element2 sector field inductively coupled plasma mass spectrometer (ICP-MS) housed at VUW's Geochemistry Laboratory. The background levels of the ICP-MS were measured by analysing the $0.2 \mathrm{~N} \mathrm{HNO}_{3}$ elution solution. These background measurements were run after every 4 to 5 samples and the counts subtracted from those of the sample during data processing. Standard bracketing using Inorganic Ventures Re standard (1000 ppm) was applied to correct for instrumental mass bias.

\section{B.1.4 Results: Comparison of Re concentrations from Re test and full analysis B.1.4.1 Terrestrial samples}

The Re concentrations from the Re test at VUW and full analysis at Durham University (determined on samples dissolved using high-temperature carius tube digestions) for the terrestrial samples broadly covary (Table S1; Fig. S1). The lack of a perfect correlation (1:1) likely relates to the low concentrations of Re in these samples, which makes precise measurements difficult, and the small sample aliquots digested in the Re test sample, if any Re heterogeneity is present in the sample. All coaly mudstones from the Farewell Formation plot below the 1:1 line (Fig. S1), indicating that the $\mathrm{Cr}_{3}-\mathrm{H}_{2} \mathrm{SO}_{4}$ digestion method used during full analysis liberated less Re content than the inverse aqua regia method used for the Re test. It has previously been suggested that the $\mathrm{Cr}_{3}-\mathrm{H}_{2} \mathrm{SO}_{4}$ digestion solution preferentially liberates 
only the hydrogenous Re content, as it does not dissolve detrital material (Selby and Creaser, 2003; Kendall et al., 2004; Rooney et al., 2011). Therefore, the full analysis Re content for the Farewell Formation samples likely reflects the hydrogenous component. The one coal sample $(\mathrm{KS}-5 / 1 \mathrm{~d})$ in the Farewell Formation sample set plots above the 1:1 line. This sample has a high TOC content $(57.9 \%)$ compared to the coaly mudstones (1.6-10.3\%; Table S1), and therefore, would likely contain less detrital Re content which would otherwise be liberated by the inverse aqua regia solution. All the other terrestrial samples (mostly coals) from the Rakopi, North Cape and Mangahewa formations were digested using inverse aqua regia in both the Re test and full analysis. This is because digestion with the $\mathrm{Cr}_{3}-\mathrm{H}_{2} \mathrm{SO}_{4}$ solution appeared to be incomplete for these samples (see Chapter 4).

Table B.1: Re concentrations for the terrestrial samples obtained from Re test method on an ICP-MS at VUW and full analysis method on an N-TIMS at Durham University.

\begin{tabular}{|c|c|c|c|c|c|}
\hline \multicolumn{6}{|c|}{ Terrestrial samples } \\
\hline & & & & Re Test & Full analysis \\
\hline Sample ID & Formation & Lithology & TOC (wt $\%)$ & $\operatorname{Re}(p p b)$ & $\operatorname{Re}(p p b)$ \\
\hline $\mathrm{Oh} 2 / \mathrm{MC} 11 \mathrm{i}$ & Mangahewa & Coal & 59.3 & 0.31 & 0.37 \\
\hline $\mathrm{Oh} 2 / \mathrm{MC} 11 \mathrm{~h}$ & Mangahewa & Coal & 74.2 & 0.45 & 0.49 \\
\hline $\mathrm{Oh} 2 / \mathrm{MC} 11 \mathrm{f}$ & Mangahewa & Coal & 77.8 & 0.12 & 0.13 \\
\hline $\mathrm{Oh} 2 / \mathrm{MC} 12 \mathrm{c}$ & Mangahewa & Coal & 79.8 & 0.08 & 0.11 \\
\hline $\mathrm{Oh} 2 / \mathrm{MC} 12 \mathrm{~b}$ & Mangahewa & Coal & 75.1 & 0.10 & 0.20 \\
\hline KS5/1a & Farewell & Coaly mst. & 2.1 & 0.55 & 0.35 \\
\hline $\mathrm{KS} 5 / 1 \mathrm{~b}$ & Farewell & Coaly mst. & 5.1 & 0.43 & 0.28 \\
\hline $\mathrm{KS} 5 / 1 \mathrm{c}$ & Farewell & Coaly mst. & 10.3 & 0.66 & 0.51 \\
\hline $\mathrm{KS} 5 / 1 \mathrm{~d}$ & Farewell & Coal & 57.9 & 0.46 & 0.50 \\
\hline KS6/A2 & Farewell & Coaly mst. & 3.5 & 0.20 & 0.16 \\
\hline KS6/A3 & Farewell & Coaly mst. & 4.6 & 0.29 & 0.17 \\
\hline KS6/A5 & Farewell & Coaly mst. & 7.5 & 0.43 & 0.31 \\
\hline KS6/A7 & Farewell & Coaly mst. & 5.1 & 0.39 & 0.24 \\
\hline KS6/A8 & Farewell & Coaly mst. & 1.6 & 0.32 & 0.19 \\
\hline $\mathrm{RF} / 4$ & Rakopi & Coal & 49.8 & 0.42 & 0.33 \\
\hline $\mathrm{PR} / 1$ & Rakopi & Coal & 55.6 & 0.23 & 0.25 \\
\hline $\mathrm{PR} / 6$ & Rakopi & Coal & 64.0 & 0.27 & 0.25 \\
\hline $\mathrm{PR} / 10$ & Rakopi & Coal & 68.4 & 0.30 & 0.37 \\
\hline $\mathrm{PM} / 3 \mathrm{M}$ & North Cape & Coaly mst. & 12.9 & 0.25 & 0.19 \\
\hline $\mathrm{PM} / 3 \mathrm{~N}$ & North Cape & Coal & 64.2 & 0.34 & 0.38 \\
\hline $\mathrm{PM} / 3 \mathrm{O}$ & North Cape & Coaly mst. & 55.1 & 0.24 & 0.26 \\
\hline $\mathrm{OP} / 1$ & North Cape & Coal & 57.0 & 0.26 & 0.28 \\
\hline
\end{tabular}




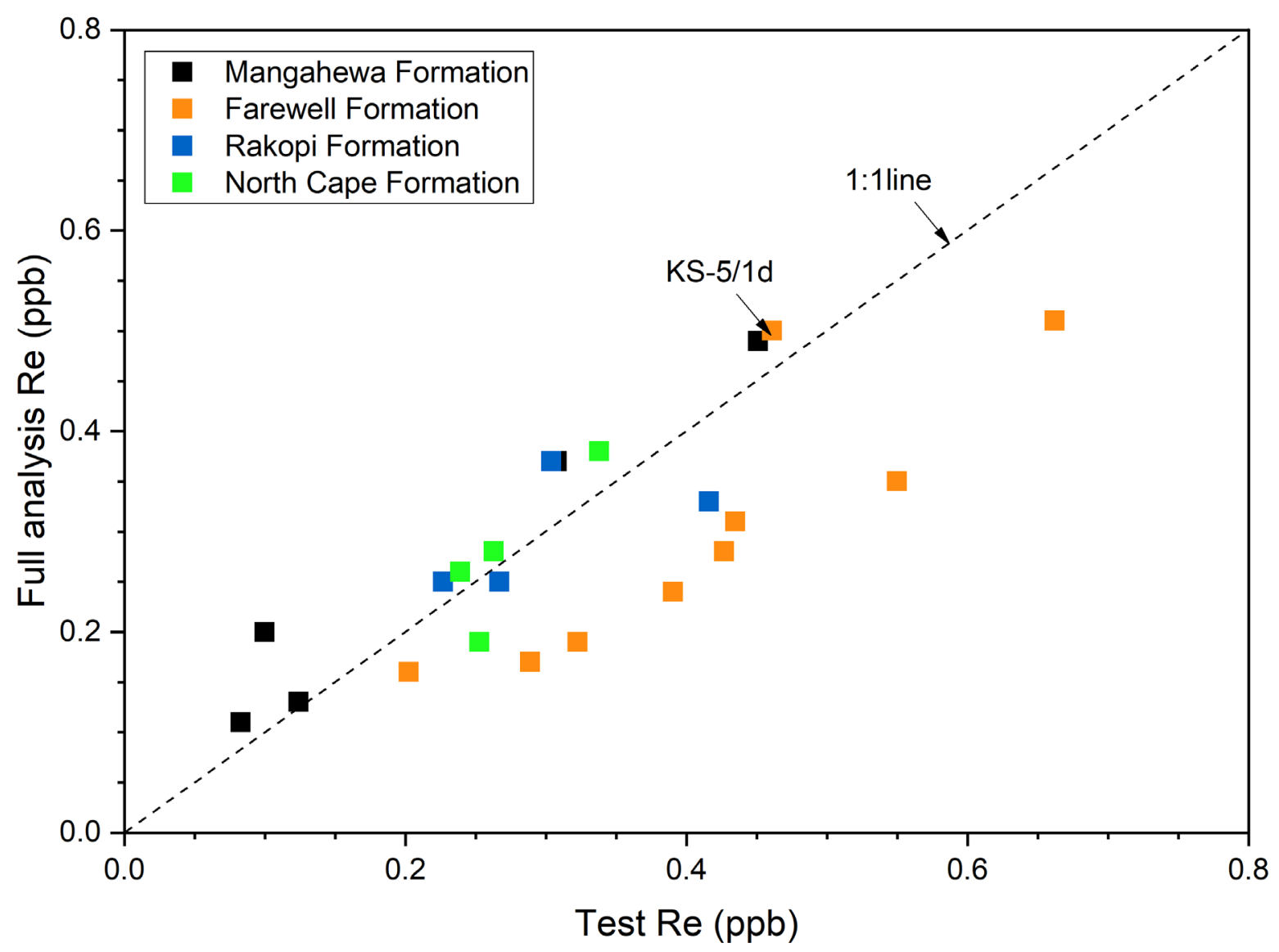

Fig. B.1. Relationships between Re concentrations for the marine samples determined during Re test (at VUW) and full analysis (at Durham University) on sample splits of the same powders.

\section{B.1.4.2 Marine samples}

The Re concentrations from the Re test and full analysis for the marine samples are in close agreement, except for two outliers, TW-49 and TW-51 (Table S2; Fig. S2). As $\mathrm{Cr}_{3}-\mathrm{H}_{2} \mathrm{SO}_{4}$ digestion method was used for the full analysis, the lower Re concentrations in the full analysis of the two outlier samples might indicate a greater contribution of detrital $\mathrm{Re}$ given that $\mathrm{Cr}_{3}-$ $\mathrm{H}_{2} \mathrm{SO}_{4}$ preferentially liberates the hydrogenous Re (Selby and Creaser, 2003; Kendall et al., 2004; Rooney et al., 2011). However, the differences in Re concentrations (53 ppb in TW-49 and $15.5 \mathrm{ppb}$ in TW-51) are too large to be ascribed to a detrital source in which crustal abundance of Re is typically less than 2 ppb (Peucker-Ehrenbrink and Jahn, 2001; Sun et al., 2003). These anomalous results are also not considered to be an artefact of heterogeneity in the sample powders because Re concentrations of replicate analyses were reproducible within $2 \%$ 
using the aqua regia method and $0.2 \%$ using the $\mathrm{Cr}_{3}-\mathrm{H}_{2} \mathrm{SO}_{4}$ method. It is suggested that these anomalous results may be related to subtle differences in the sample matrix that influenced the efficacy of the digestion methods used.

Table B.2: Re concentrations for the marine samples obtained from Re test method on an ICPMS at VUW and full analysis method on an N-TIMS at Durham University.

\begin{tabular}{|c|c|c|c|c|c|}
\hline \multicolumn{6}{|c|}{ Marine samples } \\
\hline & & & & Re Test & Full analysis \\
\hline Sample ID & Formation & Lithology & $\mathrm{TOC}(\mathrm{wt} \%)$ & $\operatorname{Re}(p p b)$ & $\operatorname{Re}(p p b)$ \\
\hline TW-51 & Waipawa & mst. & 2.8 & 71.80 & 56.30 \\
\hline TW-51 rpt & Waipawa & mst. & 2.8 & - & 56.26 \\
\hline TW-49 & Waipawa & mst. & 3.8 & 89.42 & 36.40 \\
\hline TW-49 rpt & Waipawa & mst. & 3.8 & 91.48 & 36.35 \\
\hline TW-48 & Waipawa & mst. & 3.3 & 33.89 & 35.60 \\
\hline TW-47 & Waipawa & mst. & 1.7 & 22.88 & 31.60 \\
\hline TW-38 & Waipawa & mst. & 4.5 & 23.28 & 23.50 \\
\hline TW-29 & Waipawa & mst. & 4.0 & 71.38 & 72.90 \\
\hline TW-25 & Waipawa & mst. & 2.4 & 25.81 & 26.20 \\
\hline TW-17 & Waipawa & mst. & 3.2 & 80.46 & 80.50 \\
\hline Orui-10b & Waipawa & mst. & 3.4 & 28.34 & 28.35 \\
\hline Orui-10c & Waipawa & mst. & 2.6 & 22.43 & 22.51 \\
\hline Orui-10d & Waipawa & mst. & 2.5 & 22.43 & 23.07 \\
\hline Orui-11a & Waipawa & mst. & 3.3 & 29.86 & 29.83 \\
\hline Orui-11b & Waipawa & mst. & 3.2 & 30.38 & 30.84 \\
\hline Orui-11c & Waipawa & mst. & 2.7 & 24.98 & 25.11 \\
\hline Orui-11d & Waipawa & mst. & 3.2 & 30.73 & 30.64 \\
\hline Orui-11e & Waipawa & mst. & 3.0 & 26.18 & 26.91 \\
\hline Orui-11f & Waipawa & mst. & 4.2 & 35.88 & 36.89 \\
\hline MT3.15 & Whangai & mst. & 1.1 & 4.99 & 5.02 \\
\hline MT3.16 & Whangai & mst. & 0.9 & 7.44 & 7.55 \\
\hline MT3.17 & Whangai & mst. & 0.8 & 3.65 & 3.64 \\
\hline MT3.18 & Whangai & mst. & 0.9 & 4.79 & 4.95 \\
\hline MT3.19 & Whangai & mst. & 0.9 & 11.40 & 11.83 \\
\hline
\end{tabular}




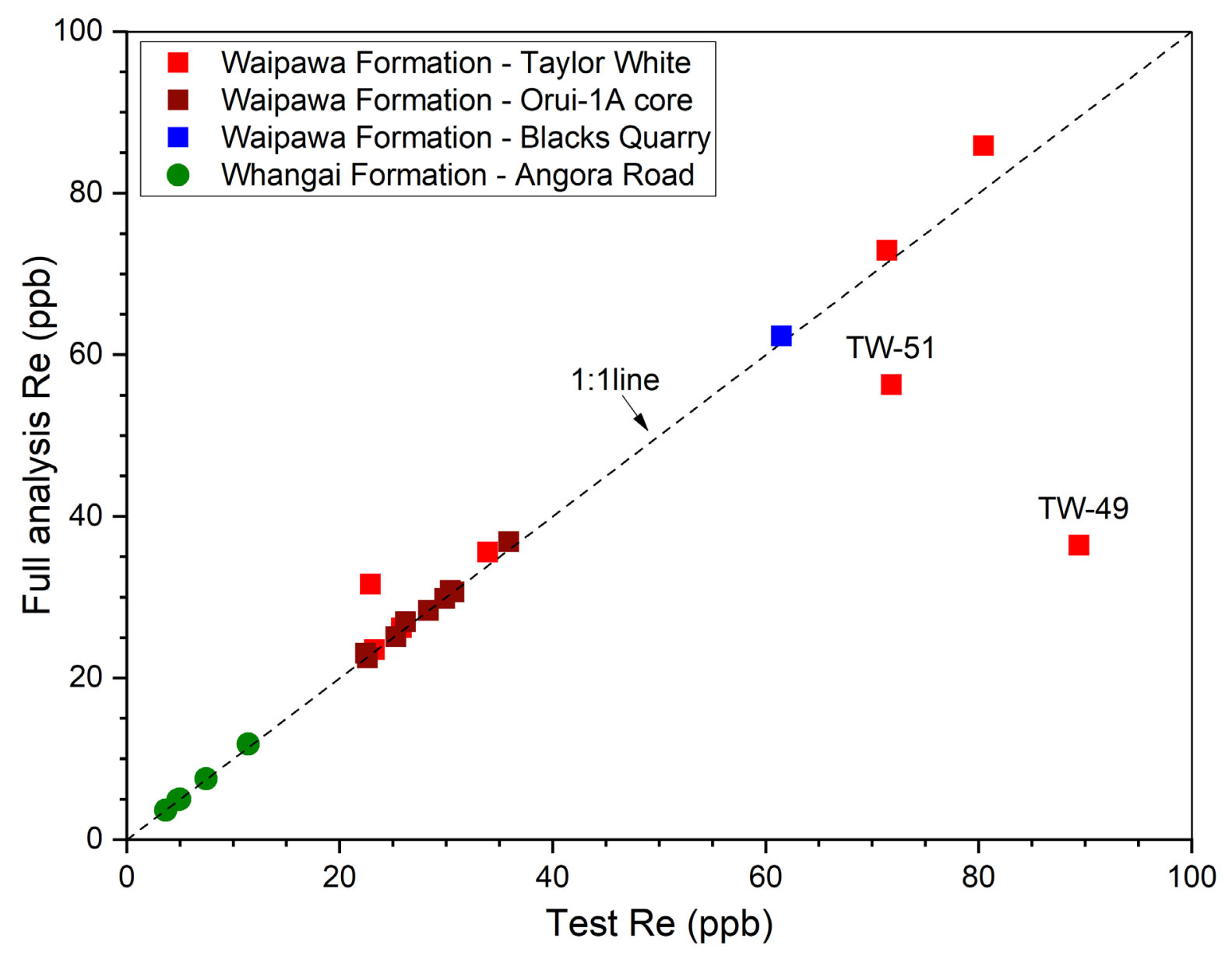

Fig. B.2. Relationships between Re concentrations for the marine samples determined during Re test (at VUW) and full analysis (at Durham University) on sample splits from the same powders. 


\section{References}

Kendall, B.S., Creaser, R.A., Ross, G.M., Selby, D., 2004. Constraints on the timing of Marinoan "Snowball Earth" glaciation by ${ }^{187} \mathrm{Re}^{187}{ }^{187}$ Os dating of a Neoproterozoic, postglacial black shale in Western Canada. Earth and Planetary Science Letters, 222: 729 740.

Peucker-Ehrenbrink, B., Jahn, B., 2001. Rhenium-osmium isotope systematics and platinum group element concentrations: Loess and the upper continental crust. Geochemistry Geophysics Geosystems, 2: doi.org/10.1029/2001GC000172.

Rooney, A.D., Chew, D.M., Selby, D., 2011. Re-Os geochronology of the NeoproterozoicCambrian Dalradian Supergroup of Scotland and Ireland: Implications for Neoproterozoic stratigraphy, glaciations and Re-Os systematics. Precambrian Research, 185: 202-214.

Selby, D., Creaser, R.A., 2003. Re-Os geochronology of organic rich sediments: an evaluation of organic matter analysis methods. Chemical Geology, 200: 225-240.

Sun, W., Bennett, V.C., Eggins, S.M., Kamenetsky, V.S., Arculus, R.J., 2003. Enhanced mantle-to-crust rhenium transfer in undegassed arc magmas. Nature, 422: 294-297.

Sykes, R., Zink, K.-G., Rogers, K.M., Phillips, A., Ventura, G.T., 2012. New and updated geochemical databases for New Zealand petroleum samples, with assessments of genetic oil families, source age, facies and maturity. GNS Science consultancy report 2012/37, GNS Science Consultancy Report, 36 pp. 